Evidence for Hard Giuon Bremsstrahlung in a Neutrino Experiment

By

Michael David Sokoloff

B.S. (University of Maryland) 1974

M.A. (University of Califormia) 1976

DISSERTATION

Submitted in partial satisfaction of the requirements for the degree of DCCIOR OF PHILOSOPHY

is

Physics

in the

GRADUATE DIVISION

OF THE

UNIVERSITY OF CAIIFORNIA, BERKELEY

Approved:
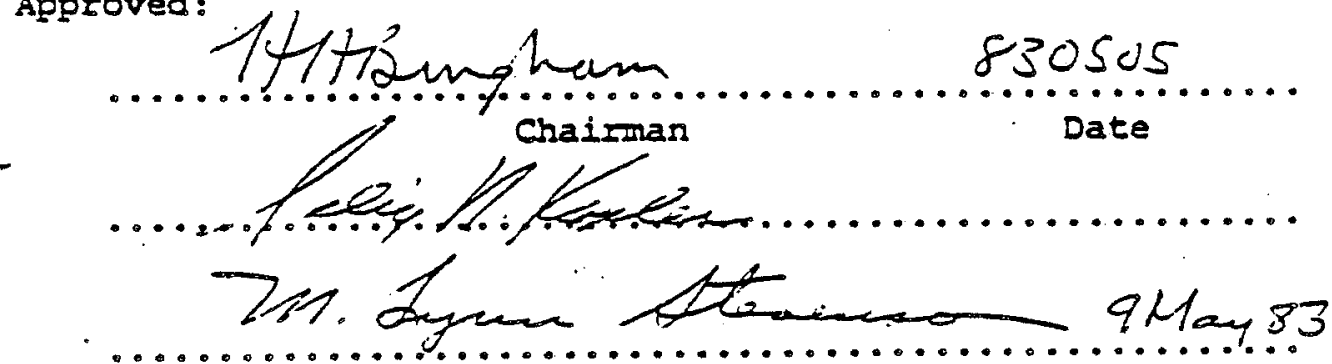


\title{
Evidence for Hard Gluon Bremsstrahlung in a Neutrino Experiment
}

\author{
Michael David Sokoloff
}

ABSTRACT

We have studied $\nu_{\mu} N$ charged current events produced in the Fermilab 15 -foot bubble chamber by neutrinos from the quadrupole triplet beam for evidence of the hard gluon bremsstrahlung process $W=q \rightarrow q g$ predicted by Quantum Chromodynamics (QCD). Hard gluon bremsstrahlung events should display three-jet structure with two jets forward (quark and gluon) and one backward (diquark) in the hadron sys. tem center-of-mass (CM). Forward hadrons are expected to carry the transverse momentum of the quarks and gluons from which they evolve. This leads to predictions of more hadron transverse momentum $\left(P_{T}\right)$ in the forward half of the $\mathrm{CM}$ than in the backward half. For hadron system invariant mass, $W$, greater than 10 $\mathrm{GeV}$, we find the difference between forward and backward mean track transverse momentum squared to be $\Delta P_{T}^{2}=\left\langle P_{T}^{2}\right\rangle_{F}-\left\langle P_{T}^{2}\right\rangle_{B}=0.047 \pm 0.011 \mathrm{GeV}^{2}$ (statistical error only). Events with high $\pi_{F}, \pi_{F}$ a measure of forward $P_{T}$, tend to have planar hadron systems and show a three-jet structure in their angular energy flow. The net number of high $\pi_{F}$ events at high $W$ exceeds that expected in two-jet models. Our observations are consistent with QCD predictions for hard gluon bremsstrahlung. The rate of three-jet events we deduce from our data corresponds to an average QCD coupling constant for the three-jet events of $\left\langle\alpha_{s}\right\rangle=0.29 \pm 0.05 \pm 0.05$ (the first error statistical, the second systematic). 


\section{Acknowledgements}

The experiment described in this thesis represents the work of many people. It is impossible to properly acknowledge the contributions made by all the technicians, scanners, physicists, engineers, and others, so let me simply say thank you to all who helped me over the years. Let me also give special thanks to the members of the Cosmic Rays group in Berkeley with whom it was always a pleasure to work.

In my years as a graduate student I learned most about how to do physics research from the individuals with whom I worked daily. Craig Ballagh, Ted Lawry, and Jeremy Lys not only did a disproportionate share of the work represented here, they also helped me discover what it means to be a physicist.

Most importantly, I want to express my appreciation to Harry Bingham who was my faculty advisor, our group leader, and a friend. The effectiveness our research group enjoyed was due in large measure to his quiet leadership, which was equal parts good fellowship, excitement, and scholarship. You can't ask for more. 
Table of Contents

Acknowledg ements ........................................................................... i

I: Introduction and Overview .......................................................................

II:- Neutrino-Nucle on Deep Inelastic Scattering Theory ................................... 4

II.1 Fundamentals of the Quark Parton Model .............................................

II.2 The QPM Charged' Current Cross Section ........................................... 5

II.3 QPM Final State Hadrons ................................................................ 8

II.4 Fundamentals of Quantum Chromodynamics ....................................... 9

II.5 Three-Jet Kinematics ..................................................................... 10

II.6 Three-Jet Differential Cross Sections …............................................... 12

II.7 Azimuthal Asymmetries in the Parton Final State .............................. 13

II.8 The Strong Coupling Constant .............................................................

III: Tae Data Sample ................................................................................... 16

III.1 Point Measurement Errors ........................................................... 16

III.2 Selection of Tracks Used in Event Reconstruction ............................ 18

III.3 Event Reconstruction Algorithm …...................................................... 20

III.4 Kinematic Reconstruction Errors …................................................... 24

IV: Monte Carlo Calculations of the Models .................................................... 26

IV.1 Generating the Weak Interaction …................................................ 26

IV.2 Generating Hadrons from Partons ….............................................. 29

IV.3 The Field and Feynman Single Quark Jet Model ................................. 29

IV.4 Chris Day's TUBES Model …............................................................ 30 

IV.5 TUBES and Phase-Space 32

V: Analyzing the Data 34

V.1 QCD Predictions for Quark and Gluon Transverse Momentum 34

V.2 QCD Predictions for Hadron TransVerse Momentum 32

V.3 $\left\langle P_{T}^{2}\right\rangle$ as a Test of QCD 36

V.4 Experimental Results for $\left\langle P_{T}^{2}\right\rangle$ 37

V.5 Event Transverse Momentum 39

V.6 Transverse Momentum Shape 44

V.7 Angular Energy Flow 48

VI: Interpreting the Data in the QCD Model .... 52

VI.1 The Three-jet Rate and $\alpha_{s}$ 52

VI.1.1 The Uncertainty in Jet Parmeterization 53

VI.1.2 The Uncertainty in Modelling the Detector 53

VI.1.3 The Uncertainty in Subtracting the Two-jet Background 54

VI.1.4 Uncertainties Which Result from Using a Neon Target 55

VI.2 Limitations of the QCD Model 56

VI.3 Event Characteristics 57

VII: Summary and Conclusions 60

References 64

Appendix A: The Neutrino-Quark Cross Section 66

Appendix B: The Sudakov Form Factor: Why and How 63

Figure Captions 71

Figures 82

Tables

$\because$ 



\section{Chapter I: Introduction and Overview}

The Quark Parton Model (QPM) and Quantum Chromodynamics (QCD) correctly describe and correlate many recent experimental observations in particle physics. QPM defines the constituents of mesons and baryons to be point-like quarks and antiquarks. QCD describes the strong interactions of these quarks in a field theory which allows infrared slavery (very strong coupling at low momentum transfer) and ultraviolet freedom (relatively weak coupling at high momentum transfer). Deep inelastic scattering experiments at SLAC in the late 1960's provided the first evidence for point-like constituents of baryons. Since then, many types of high momentum transfer experiments have been performed, and QPM has evolved to describe essentially all the results in a seif-consistent way. QCD provides a field-theoretic justification for many of the assumptions made in QPM and also predicts phenomena not required by QPM.

One of the cleanest predictions of $Q C D$ is that in very high momentum transfer processes quarks should radiate bard gluons, the exchange boson of the theory, at a rate proportional to $\alpha_{s}$, the strong coupling constant. This is analogous to the bremsstrahlung process of Quantum Electrodynamics (QED), so is called hard giuon bremsstrablung. The observation of three-jet events at PETRA is generally interpreted as the observation of such a process, specifically as $e^{+} e^{-} \rightarrow q \bar{q} g$ ( $q$ denotes quark, $\bar{q}$ denotes antiquark, $g$ denotes gluon), and provides some of the most striking evidence for QCD.

The deep inelastic scattering process analogous to $e^{+} e^{-} \rightarrow q \vec{q} g$ is $W^{ \pm}\left(\gamma^{*}\right) q \rightarrow q g\left(W^{ \pm}\right.$ denotes weak intermediate vector boson, $\gamma^{*}$ denotes virtual photon), where the remaining diquark in the target nucleon is a spectator to the weak (electromagnetic) interaction. As for $e^{+} e^{-} \rightarrow q \bar{q} g$, $W=q \rightarrow q g$ events should should show three-jet structure when viewed in the hadron system centerof-mass (CM), the three jets nominally evolving from the quark, diquark, and gluon. Essentially the same Feynman diagrams describe $e^{t^{-}} e^{-} q \bar{q} g$ and $W=q \rightarrow q g$, so to the extent that bard gluon bremsstrahlung produces three-jet events in $e^{+} e^{-}$interactions, $Q C D$ demands that $\overline{i t}$ produce threejet events in deep inelastic scattering interactions. With this motivation, we have studied high 

energy neutrino-nucleon interactions for evidence of the $W=q-q g$ process.

The particles one may observe experimentally are ordinary hadrons, not quarks or gluons. To study an underlying process such as hard gluon bremsstrahiung requires that one understand phenomenologically how quarks and gluons evolve into ordinary hadrons. Given this understanding, one may predict what a hard gluon bremsstrahlung signal will look like. Processes other than hard gluon bremsstrahlung may produce hadron final states similar to those expected for the signal. To establish a signal, one must demonstrate either that such processes do not occur or that they occur at a much lower rate than is observed. In this sense the interpretation of our data is model dependent.

We have analyzed our data in the context of QPM and QCD. Hard gluon bremsstratilung should produce quarks and gluons at high transverse momentum in the forward direction of the hadron system. These quarks and gluons should evolve into high transverse momentum hadrons, and these are the handle we use in looking for a hard gluon bremsstrahiung signal. Because other processes also produce high transverse momentum hadrons, we have studied variables and distributions most likely to distinguish between models.

The analysis which follows is presented in a structure that emphasizes the modeis being tested: Chapter II introduces QPM and QCD at the parton level. Differential neutrino-nucleon crosssections are derived using QPM, and the hard gluon bremsstrahlung cross-section is presented as a QCD first order perturbative effect. Relevant parton and event variables are defined.

Chapter III introduces the data sample to be analyzed. The detector, track measurement pro cedures, kinematics reconstruction, and attencant uncertainties are discussed.

Chapter IV describes the Monte Carlo computer programs used to compare the data with the models and to study instrumental effects. The evolution of quarks, diquarks, and gluons into final state hadrons is described, and various quark and gluon distributions in hard giuon bremsstrahlung events are calculated for the energy spectrum of this experiment.

Chapter $\mathrm{V}$ contains the data analysis. The variables studied are defined and expiained in the context of QPM and QCD. Full Monte Carlo calculations including instrumental effects are 
compared with the data. Some model independent conciusions are drawn.

Chapter VI analyzes the results of chapter V in terms of the QPM and QCD models embodied in the Monte Carlo programs. Systematic errors affecting the correspondence between the data and the models are also discussed.

Chapter VII summarizes the results and presents the conclusions.

Earlier versions of the analysis presented in this work were reported at several conferences and in Physical Review Letters'. Greg Swider's Ph.D. thesis ${ }^{2}$ describes a related analysis of a smaller data sample from this experiment. The results of other analyses of data from this experiment have also been reported, and the interested reader may find further details and related results in those works ${ }^{3}$. 


\section{Chapter II: Neutrino-Nucleon Deep Inelastic Scattering Theory}

The naive Quark Parton Model (QPM) describes deep inelastic lepton-nucleon scattering as a two step process. First, on a short time scale, the incident lepton scatters from a quark within the target nucieon, leaving the remaining diquark as a spectator to this interaction. Then, on a longer time scale, the quark and diquark evolve into hadron jets, each of limited transverse momentum. Quantum Chromodynamics ( $Q C D)$ is a field theory of strong interactions which justifies many of the assumptions of QPM and which also predicts new phenomena. Among these is the emission of hard gluons when quarks are greatly accelerated. These gluons will evolve into limited transverse momentum jets, similar to those of the quarks and diquarks, giving the hadron systems in such events a three-jet structure.

\section{Fundamentals of the Quark Parton Model}

In the simplest version of the Quark Parton Model (QPM), baryons consist of three valence quarks and some sort of give which binds the quarks together. Quarks are point-like, spin $\frac{1}{2}$ particles which come in several flavors. Each flavor has a characteristic electric charge which is an integral multiple of $\pm \frac{1}{3} e$. Five flavors of quark have been identified experimentally (see Table I1). Protons and neutrons contain up (u) and down (d) quarks only, the proton being a uud combination and the neutron being a udd combination.

Inside a target nucleon each quark can also be characterized by its longitudinal momentum fraction, $\xi$. This means that its four momentum, $P_{q}$, can be treated as a fraction of the nucleon's four momentum, $P_{N}$, according to the prescription

$$
P_{q}=\xi \cdot P_{N}
$$

whenever there is a longitudinal direction defined by the interaction and the energy scales of the interaction are much greater than the proton mass. In such cases $\xi$ is a property of the quark and not of the interaction; the quarks of flavor $q$ inside a nucleon are then described by a distribution function, $q(\xi)$, which gives the number density and is independent of the nature or energy of the interaction. 
The longitudinal momentum fraction of a quark can be derived by assuming that the quark acts as a free particle. In the lab the quark four momentum can be written as

$$
P_{q}^{l a b}=\left(E_{q}, \vec{P}_{q}^{T}, P_{q}^{\|}\right)
$$

where $E_{q}$ is the energy of the quark $\left(=\left[m_{q}^{2}+\vec{P}_{q}^{2}\right]^{\frac{1}{2}}\right), P_{q}^{\|}$is the component of quark momentum along the longitudinal direction, and $\vec{P}_{q}^{T}$ is the two dimensional component of the quark momentum perpendicular to that direction. The nucleon's lab four momentum is

$$
P_{N}^{l a b}=\left(m_{N}, \overrightarrow{0}\right) \text {, }
$$

as it is defined to be at rest in the lab. Boosting by $\beta$ in the longitudinal direction gives new longitudinal momenta:

$$
P_{q}^{\|}(\beta)=\gamma\left(P_{q}^{\|}+\beta E_{q}\right)
$$

and

$$
P_{N}(\beta)=\gamma\left(\beta m_{N}\right) \text {. }
$$

In the limit $\beta \rightarrow 1$ the ratio of these longitudinal momenta becomes

$$
\xi=\frac{P_{q}^{\|}(1)}{P_{N}^{\|}(1)}=\frac{P_{q}^{\|}+E_{q}}{m_{N}} .
$$

In the same limit energy is equal to momentum and transverse momentum is negligibly small, so that equation equation II. I becomes a fair approximation and longitudinal momentum fraction is a property of the quark.

The glue which binds quarks together into baryons has a range and a momentum scale characterized by the size of the proton. QPM assumes that for shorter distances and higher momenta the glue fails to interact with the quaris, so that for the duration of quick interactions the quarks act as free particles. This is sometimes called ultraviolet freedom. Assuming this is the case for deep inelastic scattering, neutrino-nucleon cross sections can be calculated by considering the neutrino-quark interactions and summing over the quarks which constitute the nucleon.

\section{The QPM Charged Current Cross Section}

Figure II-1 shows the Feynman diagram for neutrino-quark charged current interactions. The neutrino emits a charged boson, the $W^{+}$, and turns into a muon. The boson is absorbed by a down 
quark and turns into a (Cabibbo rotated) up quark. For the energies of this experiment the effect of the boson propagator is negligible, so it suffices to compute the matrix element from the point-like V-A interaction of figure II-2. This gives ${ }^{4}$

$$
\left|M^{2}\right|=64 G^{2}\left(P_{A} \cdot P_{B}\right)\left(P_{C} \cdot P_{D}\right) .
$$

For neutrino quark interactions $P_{A}$ is the neutrino four momentum $P_{y}, P_{B}$ is the target quark four momentum $P_{q}, P_{C}$ is the muon four momentum $P_{\mu}$, and $P_{D}$ is the recoil quark four momentum $P_{q^{\prime}}$. Determining the matrix elements for neutrino-antiquark, antineutrino-quark, and antineutrino. antiquark interactions simply requires permuting the indices of the four vectors according to the usual Feynman rules for particles and antiparticles.

The total neutrinomuark cross section with $\left|M^{2}\right|$ from equation II.6 is (see appendix A):

$$
\sigma(\nu q)=\frac{G^{2}}{\pi} \dot{s}
$$

where

$$
\hat{s}=\left(P_{\nu}+P_{q}\right)^{2}=\left(P_{\nu}+\xi P_{N}\right)^{2} \approx 2 m_{N} \xi E_{\nu} .
$$

Neutrino-nucleon cross sections are found by summing over the target quarks in the nucleon according to their number density, $q(\xi)$ :

$$
\sigma(u N)=\frac{2 G^{2} m_{N} E_{\nu}}{\pi} \int \xi q(\xi) d \xi
$$

Given $E$, and $\xi$, the Lorentz boost from the lab to the neutrino-quark center-of-momentum (CM) system is defined. The interaction is assumed to be elastic so that the only kinematic freedom remaining is the scattering angle, $\theta^{C M}$, between the neutrino and the muon directions. With the three variables, $E_{y}, \xi$, and $\theta^{C K}$. the lepton (and quark) kinematics are fully determined. Of these, only $E_{\mathrm{y}}$ is an observable quantity. Two Lorentz scalars are commonly used to describe deep inelastic scattering events:

$$
x_{B j} \mp-q^{2} / 2 P_{N} \cdot q
$$

and

$$
y=P_{N} \cdot q / P_{N} \cdot P_{\nu}
$$

where $q \mp P_{\nu}-P_{\mu}$ is the four momentum transfer. Assuming that neutrinoquark interactions are 
(quasi-) elastic (that the muon and quark masses are negligible), these expressions are evaluated in the neutrino-quark CM system to give:

$$
x_{B j}=\xi
$$

and

$$
y=\frac{1}{2}\left[1-\cos \left(\theta^{C M}\right)\right]
$$

Note that for inelastic neutrino-quark interactions, such as charm production and hard gluon bremsstrahlung, the final state muon will have less energy in the neutrino-quark CM than it would have had in a (quasi-) elastic interaction, so $x_{B j}<\xi$. The neutrino-quark differential cross section as a function of $\theta^{C M}$ is independent of $\dot{s}$ (see appendix A); its differential cross section in $s$ factors simply, according to the calculation of equation II.8, into a form proportional to the product of $E_{y}$ and $\xi$. Thus the neutrino-nucleon cross section grows linearly with $E_{y}$ while its $x_{B j}$ and $y$ dependences are independent of $E_{y}$. Kinematic variables which have no inherent energy scale and whose distributions remain fxed independently of the energy of the interaction are called scaling variables. $x_{B j}$ and $y$ are scaling variables in QPM.

In a somewhat more sophisticated version of the model baryons contain quark-antiquark pairs in addition to the valence quarks and the glue. These pairs contribute no net quantum numbers to a baryon, and their quarks and antiquarks have softer $x_{B_{j}}$ distributions than do the valence quarks. They are reminiscent of a Fermi sea and are of ten referred to as sea quarks and antiquarks. This nomenclature can be confusing when one also discusses charmed quarks as these are commonly called c-quarks. For this reason we will denote elements of these pairs as ocean quarks and antiquarks. Neutrinos interact with ocean quarks just as they do with valence quarks. The neutrinoantiquark cross section is different, however. The angular distribution in the neutrino-antiquark $\mathrm{CM}$ system varies like $\left(1+\cos \theta^{C M}\right)$ due to the $\mathrm{V}-\mathrm{A}$ structure of the weak current (see appendix $\mathrm{A}$ ). In the lab system this translates into a cross section which depends on $y$ in proportion to $(1-y)^{2}$. Therefore the neutrino nucleon cross section looks like:

$$
\frac{d \sigma(\nu N)}{d \xi d y d E} \propto E_{y} \int\left[\xi q(\xi)+(1-y)^{2} \xi \bar{q}(\xi)\right] d \xi .
$$


The kinematic variables most commonly used to describe neutrino events, other than neurtrino energy, are energy transfer, $\nu$, four momentum transfer squared, $-Q^{2}$, and hadron system invariant mass squared, $W^{2}$. In terms of the scaling variables they are:

$$
\begin{gathered}
\nu=y E_{\nu} \\
Q^{2}=2 m_{N} E_{y} y x_{B j} \\
W^{2}=2 m_{N} E_{y} y\left(1-x_{B j}\right)+m_{N}^{2} .
\end{gathered}
$$

\section{QPM Final State Hadrons}

During the weak interaction the diquark, meaning the nucleon minus the target quark, is a passive spectator. After the weak interaction the diquark and the struck quark form a hadron system whose four momentum is

$$
P_{H}=\left(q+P_{N}\right)
$$

and whose invariant mass squared is $W^{2}$. In the hadron CM system the quark and diquark are initially moving with equal and opposite momenta of magnitude $W / 2\left(W=\sqrt{W^{2}}\right)$ along the direction $\dot{q}$ defined by the spatial components of $q$. The quark is moving forward and the diquark is moving backward. The underlying process by which these partons (the quark and the diquark) evolve into observable hadrons is not known. However, the statistical properties of hadron systems in leptoninitiated processes $\left(e^{+} e^{-}, e N, \nu N\right)$ are described well by assuming that each parton turns into a jet of hadrons, ie., a collection of hadrons moving roughly in the direction of the the original parton.

In jet models, the longitudinal momentum of a parton is partitioned among the hadrons of its jet according to fragmentation functions $D_{q}^{h}(z)$, the mean number of hadrons of type $h$ and momentum fraction $z$ (per $d z$ ) in a jet initiated by a parton $q$ with high momentum. These hadrons are produced with transverse momenta characteristic of the strong interaction's range (several hundred $\mathrm{MeV}$ ). Some of the hadrons produced by parton fragmentation are stable hadrons such as pions and kaons. Others are short-lived such as $\rho^{\prime} s$ and $K^{* \prime} s$. These decay into stable particies which are observed as part of the jet. Such a model for single quark jets has been parameterized by Field and Feynman 5 . C. Day has expanded this in his UUBES $^{6}$ program to describe two and three-jet events with diquarks and gluons, as well as quarks, for initial partons. We have incorporated TUBES into 
our Monte Carlo simulation for this experiment, which is described more fully in Chapter IV.

\section{Fundamentals of Quantum Chromodynamics}

Quantum Chromodynamics (QCD) is a field theory which describes the strong interactions of colored (hadronically charged) quarks. There exist three color states for quarks and the forces among them are mediated by vector gluons. The Lagrangian is invariant under $\mathrm{SU}_{3}$ (color), which is a non-Abelian group. Giuons also carry color and thus couple to each other as well as to quariks. The current status of QCD has been nicely summarized by R. D. Field ${ }^{7}$ :

Although the theory is well defined, preciseiy what it predicts is not yet clearly known. For example, it is not known if the theory actually confines quarks and gluons within hadrons nor has the spectrum of hadronic states been calculated. At present the mathematical complexities are too great. However, at very high energy or momentum transfer, $Q$, the theory is asymptotically free; the effective coupling between quarks and gluons decreases with increasing $Q^{2}$. As emphasized by Politzer, this permits calculation of those parts of a process involving high $Q^{2}$ by the use of perturbation theory $\cdots$. Yet every real process involves both high and low $Q^{2}$ together and precisely how to separate these parts is just becoming clear.

Perturbative corrections to the deep inelastic scattering process of figure II-1 have been calculated to order $\alpha_{s}$ (the running coupling constant of $Q C D$ ) by several authors. The account given by Peccei and Ruck ${ }^{8}$ is particularly lucid and complete. We will adopt their notation and refer interested readers to their paper for details of the differential cross section calculations.

The only quark cross sections that enter in deep inelastic seattering, to $O\left(\alpha_{s}\right)$, are those contributed by the Feynman diagrams of figures II-3 and II-4. The non-perturbative diagram, II-3a, is exactly that used in QPM calculations. The diagrams with virtual gluon corrections, figures II-3b, have initial and final states indistinguishable from those of the unperturbed diagram. Therefore the amplitudes of II-3a and II-3b are added to compute the cross section for exchange-boson plus quark goes to quark $\left(W^{ \pm} q \rightarrow q\right)$. The diagrams with real gluon emission corrections, shown in II-3c, each have a quark in the initial state and a quark plus a gluon in the final state. Their amplitudes are added to compute the exchange-boson plus quark gces to quark plus gluon ( $W \neq q \rightarrow q g$ ) cross section. Initial state gluons will also contribute to the deep inelastic cross section via the mechanism of II-4. A quark or antiquark from gluon dissociation scatters weakly, leaving both the quark and antiquark as final state partons. The amplitudes of the two diagrams shown are added to compute the cross 
section for exchange-boson plus gluon goes to quark plus antiquark ( $W=g \rightarrow q \bar{q}$ ). The $W=g \rightarrow q \bar{q}$ contribution to the high parton transverse momentum cross section is small compared to the total high parton transverse momentum cross section ${ }^{8}$, so we shall consider the $W^{ \pm} q \rightarrow q g$ only.

\section{Three-Jet Kinematics}

Three-jet kinematics requires two variables more than two-jet kinematics to describe the additional degrees of freedom. The three-jet cross section ( $W=q \rightarrow q g$ ) in $Q C D$ (almost) scales in the variables $x_{p}$ and $z_{q}\left(z_{g}\right)$, which are defined as the Lorentz invariants:

$$
\begin{gathered}
x_{p} \mp \frac{x_{B j}}{\xi} \\
z_{q(g)} \mp \frac{P_{q} \cdot P_{q^{\prime}(g)}}{P_{q} \cdot q}=\frac{P_{N^{\prime}} \cdot P_{q^{\prime}(g)}}{P_{N^{\prime}} \cdot q}
\end{gathered}
$$

where $P_{q^{\prime}(g)}$ is the momentum of the final state quark (gluon). The interpretation of $z_{q(g)}$ in the lab frame is easy, as it is computed explicitly to be the quark's (gluon's) fraction of the total energy transferred to the hadron system, $z_{q(g)}=E_{q(g)} / \nu$. Thus, $z_{q}+z_{g} \approx 1$. The variable $x_{p}$ describes the inelasticity of the neutrino-quark interaction. The invariant mass squared of the recoiling quark plus givon system is:

$$
M_{q g}^{2}=2 m_{N} \nu \xi\left(1-x_{p}\right)
$$

As an exercise in understanding three-jet kinematics one can consider a three-jet $\left(W^{-} q \rightarrow q g\right)$ event's geometry in the hadron CM system. Demanding only conservation of four-momentum, one calculates that the exchange boson and the target nucleon initially approach each other with four momenta (neglecting the nucleon mass):

$$
q=\left|P_{N}^{C M}\right|\left(1-2 x_{B j}, 0,0,1\right)
$$

and

$$
P_{N}^{C H}=\left|P_{N}^{C M}\right|(1,0,0,-1)
$$

where

$$
\left|P_{N}^{C M}\right|^{2}=\frac{m_{N}{ }^{\nu}}{2\left(1-x_{B j}\right)}
$$

From equation II. I the target quark therefore has four momentum: 


$$
P_{q}^{C M}=\left|P_{N}^{C M}\right|(\xi, 0,0,-\xi)
$$

From conservation of energy and momentum the final state partons $(f=q, g)$ of the weak interaction have four momenta:

$$
P_{f}=\left|P_{f}\right|\left(1, \sin \theta_{f} \cos \phi_{f}, \sin \theta_{f} \sin \phi_{f}, \cos \theta_{f}\right)
$$

where

$$
\left|P_{f}\right|=\left|P_{N}^{C M}\right|\left(z_{f}(1-\xi)+\left(\xi-x_{B j}\right)\right)
$$

and

$$
\cos \theta_{f}=\frac{z_{f}\left(1+\xi-2 x_{B j}\right)-\left(\xi-x_{B j}\right)}{z_{f}(1-\xi)+\left(\xi-x_{B j}\right)} .
$$

The diquark four momentum is the nucleon four momentum minus the target quark four momentum:

$$
P_{q q}=\left|P_{N}^{C M}\right|(1-\xi, 0,0,-1+\xi) \text {. }
$$

The azimuthal angle of the quark, $\phi_{q}$, is measured about $\dot{q}$ such that the azimuthal angle of the muon is 0 and the azimuthal angle of $\vec{P}_{\nu} \times \vec{P}_{\mu}$ is $+\frac{\pi}{2}$.

To the extent that $Q C D$ predicts scaling in $\xi, x_{p}$, and $z_{q}$, it also predicts scaling in $\theta_{f}$. Parton transverse momentum (recoil quark or gluon) grows with energy for fixed $\xi, x_{p}$, and $z_{q}$ :

$$
P_{T P}=\left|P_{f}\right| \sin \theta_{f}=\left[2 m_{N} \nu z_{q} z_{z} \xi\left(1-x_{p}\right)\right]^{1 / 2} .
$$

Both $Q^{2}$ and $W^{2}$ are proportional to $\nu$, so $P \frac{2}{T P}$ grows linearly with either $Q^{2}$ or $W^{2}$ for these assumptions. However, small vaiues of $x_{p}$, which give large $P_{T P}$, turn high $\xi$ events into lower $x_{B j}$ events, and therefore (see equation [II.14b]) into lower $Q^{2}$ events. This anti-correlation originates in the space-like nature of the four-momentum transfer, $q$. As gluon bremsstrahlung becomes harder, rela tively more energy (as opposed to three-momentum) must be transferred from the lepton vertex to the hadron vertex in order to give the quari-gluon pair its large mass. This contributes a positive (time-like) mass ${ }^{2}$ to a negative (space-like) $q^{2}$, which brings its magnitude closer to zero, and therefore reduces the observed (positive) $Q^{2}$. Thus the correlation between $P_{T P}$ and $Q^{2}$ is not as great as that between $P_{T P}$ and $W^{2}$. 


\section{Three-Jet Differential Cross Sections}

The parton differential cross sections from QCD are usefully written with the QPM dynamics (equation II.13) factored out. In this case they give the relative probability for producing a particular hadron $\mathrm{CM}$ geometry, assuming an initial quark has been struck (with $E_{y}, \xi$, and $y$ specified). The differential cross section for producing a parton $p$ can be written as ${ }^{8}$ :

$$
d \sigma_{p}=d \sigma_{p}{ }^{(0)}+\cos \phi d \sigma_{p}{ }^{(1)}+\cos 2 \phi d \sigma_{p}{ }^{(2)}
$$

where $\phi$ is the relative azimuthal angle between the produced parton and the lepton plane. Parton transverse momentum cross sections can be determined from $d \sigma_{p}{ }^{(0)}$ only; both $d \sigma_{p}{ }^{(1)}$ and $d \sigma_{p}{ }^{(2)}$ are independent of $\phi$ so the $\cos \phi$ and $\cos 2 \phi$ terms do not contribute to the integral of $d \sigma_{p}$ over $\phi$.

The term $d \sigma_{p}{ }^{(0)}$ can be decomposed into a general form with Transverse(T), Scalar(S), and Interference(I) terms:

$$
\left(d \sigma_{p}{ }^{(0)}\right)^{y / \bar{\nu}}=\frac{1}{2}\left(1+(1-y)^{2}\right) d \sigma_{p}{ }^{T}+(1-y) d{\sigma_{p}}^{S} \pm y\left(1-\frac{y}{2}\right) d \sigma_{p}{ }^{l} .
$$

The Transverse and Scalar terms are the same in electron and muon scattering as in neutrino scatter. ing when the appropriate electric charges replace the weak charge.: The Interference term, which enters with opposite signs for neutrino and antineutrino scattering, disappears for electron and muon scattering. Although we shall not discuss electro-magnetic scattering further, most of the theoretical results do carry over directly.

For the diagrams of figure II-3 one finds:

$$
\begin{gathered}
d \sigma_{q}{ }^{T}=d \phi_{q g} \frac{2 \alpha_{s}}{3 \pi}\left[\frac{z_{q}^{2}+x_{p}^{2}}{\left(1-x_{p}\right)+\left(1-z_{q}\right)_{+}}+2\left(x_{p} z_{q}+1\right)\right] \\
d \sigma_{q}{ }^{s}=d \phi_{q g} \frac{2 \alpha_{s}}{3 \pi}\left[4 x_{p} z_{q}\right] \\
d \sigma_{q}{ }^{I}=d \phi_{q g} \frac{2 \alpha_{s}}{3 \pi}\left[\frac{z_{q}^{2}+x_{p}^{2}}{\left(1-x_{p}\right)_{+}\left(1-z_{q}\right)_{+}}+2\left(x_{p}+z_{q}\right)\right]
\end{gathered}
$$

when $x_{p} \neq 0$ and $z_{q} \neq 0$ and where $d \phi_{q g}$ is the phase space element that enters in $q \rightarrow q g$ scattering:

$$
\begin{gathered}
d \phi_{q g}=d x_{p} d y d z_{q} \delta\left(1-z_{q}-z_{g}\right) d \cos \theta_{q} d \cos \theta_{g} \\
\times \delta\left(\cos \theta_{q}-h\left(z_{q}\right)\right) \delta\left(\cos \theta_{g}-h\left(z_{g}\right)\right) d \phi_{q} d \phi_{g} \frac{1}{2 \pi} \delta\left(\phi_{q}-\phi_{g}-\pi\right) .
\end{gathered}
$$


The function $h\left(z_{f}\right)$ is the expression found on the right side of equation II-24. The functional $\frac{1}{(1-x)_{+}}=\frac{1}{(1-x)}$ for $x \neq 1$, so the terms in equation II-29 appear to be well defined mathematically. In fact, this is only true to the extent that one integrates over all $x_{p}$ and $z_{q}$, including the limits where they equal zero. In order to have a physically sensible result one must damp these terms in the limits $x_{p} \rightarrow 1$ and $z_{q} \rightarrow 1$. The methods used for the calculations presented in the remainder of this work are to multiply the three-jet cross section, $d \sigma_{p}$ (equation II.27), by the Sudakov form factor ${ }^{2}$, and to treat three-jet events with small quark-gluon invariant masses as twojet events. The Sudakov form factor is discussed in Appendix B, and the small mass cut-off is discussed in sections IV.1 and IV.4, where details of the Monte Carlo are presented. In the limits $x_{p} \rightarrow 1$ and $z_{q} \rightarrow 1$ three-jet events become indistinguishable from two-jet events. Given the current state of knowledge and the limited statistics of this experiment, the approximations made for the region of phase-space near $\left(x_{p}, z_{q}\right)=(1,1)$ should suffice.

\section{Azimuthal Asymmetries in the Parton Final State}

The terms which describe the azimuthal asymmetries in $d \sigma_{q}$ are

$$
\begin{gathered}
\left(d \sigma_{q}{ }^{(1)}\right)^{n / \nu}=d \phi_{q g} \frac{4 \alpha_{s}}{3 \pi} y\left[\frac{(1-y) x_{p} z_{q}}{\left(1-x_{p}\right)\left(1-z_{q}\right)}\right]^{1 / 2} \\
\quad x\left[\left(1-\frac{2}{y}\right)\left(1-z_{q}-x_{p}+2 x_{p} z_{q}\right) \pm\left(1-x_{p}-z_{q}\right)\right]
\end{gathered}
$$

and

$$
\left(d \sigma_{q}{ }^{(2)}\right)^{\nu / \bar{v}}=d \phi_{q g} \frac{4 \alpha_{s}}{3 \pi}\left[(1-y) x_{p} z_{q}\right] .
$$

A positive value for $d \sigma_{q}{ }^{(1)}$ would indicate that the projection onto the lepton plane of the produced quark's momentum tends to lie closer to (makes a smaller angle with) the muon direction than does that of the momentum transfer, $\hat{q}$. A negative value for $d \sigma_{q}{ }^{(1)}$ would indicate that the quark tends to lie further from the muon than does $\vec{q}$. Experimentally, determining the azimuthal angle of the quark, $\phi_{q}$, is not straight forward. The giuon that is produced along with the quark will have equal and opposite transverse momentum, so $\phi_{g}=\phi_{q}+\pi$. To the extent that gluon jets look like quark 
jets, $\cos (\phi)=-\cos (\phi+\pi)$ says there can be no $\cos (\phi)$ asymmetry in the final hadron system. However, quark jets have net electric charge while gluon jets do not, and the three-jet cross section (equations II.28, II.29, and II.30) predicts that quark jets are typically more energetic than gluon jets $\left(z_{q}>z_{g}\right)$. These features of the theory may allow one to separate statistically quark and gluon jets.

The $\cos 2 \phi$ part of $d \sigma_{q}, d \sigma_{q}{ }^{(2)}$, has maxima at $\phi=0$ and $\phi=\pi$ and minima at $\phi= \pm \pi / 2$. It describes the extent to which quark momenta lie in the lepton plane rather than out. Here the gluon works with the quark to define a hadron plane whose angle with respect to the lepton plane is $\phi_{H}$. With regard to the $\cos 2 \phi$ asymmetry, measuring $\phi_{H}$ is as good as measuring $\phi_{q}$ since $\cos (2[\phi+\pi])$ $=\cos (2 \phi)$, ie., $\cos 2 \phi$ does not distinguish between $\phi_{q}=\phi_{0}\left(\phi_{g}=\phi_{0}+\pi\right)$ and $\phi_{q}=\phi_{0}+\pi\left(\phi_{g}=\phi_{0}\right)$. Thus, in principle, one can measure $d \sigma_{q}{ }^{(2)}$ more directly than $d \sigma_{q}{ }^{(1)}$. A numerical evaluation of both azimuthal asymmetry parameters, given the beam spectrum of this experiment, is presented in chapter IV, where the question of detecting such signals is also discussed.

\section{The Strong Coupling Constant}

In equation II.29 each term is proportional to the strong coupling constant, $\alpha_{s}$. Perturbative QCD predicts that $\alpha_{g}$ is a running coupling constant; its value depends on the momentum scale, $\mu^{2}$, of the interaction, and is usually written as

$$
\alpha_{s}\left(\mu^{2}\right)=\frac{12 \pi}{\left(33-2 N_{F}\right) \ln \left(\mu^{2} / \Lambda_{\mu}^{2}\right)}
$$

where $N_{F}$ is the number of quark flavors contributing to the renormalization of $\alpha_{s}$ and $\Lambda_{\mu}^{2}$ is a scale parameter whose value must be determined experimentally. Unfortunately, no one knows whether the argument of $\alpha_{s}$ should be the invariant momentum transfer, the parton transverse momentum, the neutrino plus quark invariant mass, the recoil quark plus gluon invariant mass, or some other quantity. Such variables approximately scale together, so one may equally well choose any of them and choose the parameter $\Lambda^{2}$ accordingly. For example, one can use either $Q^{2}$ or $W^{2}$. In this experiment $\left.\left\langle W^{2}\right\rangle \approx 4<Q^{2}\right\rangle$, so taking $\Lambda_{W^{2}}^{2}=4 \Lambda_{Q^{2}}^{2}$ would give the same average $\alpha_{s}$ for these two possibilities. The calculations presented throughout this work use the invariant mass squared of the 
recoil quark plus gluon, $M_{q g}^{2}$, as the argument of $\alpha_{5}$ and take $\Lambda_{q g}^{2}=0.6 \mathrm{GeV}^{2}$, except where otherwise noted. Because the Sudakov form factor damps the three-jet cross section, the effective value of $\Lambda_{98}^{2}$ at high parton transverse momentum is only $0.24 \mathrm{GeV}^{2}$. This is determined by calculating the value of $\Lambda_{q g}^{2}$ required in equation II.32 when $\left\langle\alpha_{s}\right\rangle$ and $\left\langle M_{q g}^{2}\right\rangle$ for hard three-jet events (as deter. mined using Monte Carlo calculations) are used for $\alpha_{s}$ and $M_{q g}^{2}$. These average values are discussed at the end of Chapter VI.

The other parameter which enters in the formula for $\alpha_{s}$ is the number of quark flavors, $N_{F}$, which contribute to the renormalization of the quark and gluon lines in the Feynman diagrams of figure II-1. These contributions come from fermion loops such as that shown in figure II-5. The calculation of these contributions for equation II-32 assumes the quarks are massless, which is not true. Up, down, and strange quarks have masses which are small compared to the momentum scales of figure II-1 found in hard gluon bremsstrahlung events. They contribute to the renormalization almost as if they were massless. Bottom quarks have a mass which is large on this scale, so they do not contribute to the renormalization. Charmed quarks have a mass which is comparable to the invariant mass of the recoil quark plus gluon, $M_{g g}$, and one expects their contribution to the renormalization to be damped relative to that of the light quarks. The calculations presented here use $N_{F}$ = 3; using a somewhat larger value to account for the contributions of charmed quarks would produce negligible changes in the results. 


\section{Chapter III: The Data Sample}

The data for this analysis come from Fermilab experiment E546. Neutrinos from the quadrupole triplet beam interacted in the 15 foot bubble chamber, which was filled with a $47 \%$ atomic Neon-Hydrogen mixture of density $0.56 \mathrm{gms} / \mathrm{cm}^{3}$. The radiation length of this liquid was $54 \mathrm{~cm}$ and the (pion) interaction length $198 \mathrm{~cm}$. The radius of the bubble chamber was 1.8 meters, so approximately $80 \%$ of the gammas converted into electron-positron pairs in the chamber and within $110 \mathrm{~cm}$ of the primary vertex, and half the charged hadrons interacted before exiting. Charged tracks were bent by a $30 \mathrm{kGauss}$ magnetic field ( $1 \mathrm{GeV} \approx 1$ meter radius of curvature) and filmed in three views. Track reconstruction was performed using the geometry programs TVGP and HYDRA. Muons were identified by extrapolating the trajectories of tracks which left the bubble chamber without visible interactions (called leaving tracks) through the External Muon Identifier ${ }^{10,11}$ (EMI) and demanding a two-plane confidence level of at least 1\%. The EMI consisted of 3-5 absorption lengths of magnet coil and zinc absorber, followed by the first plane of multi-wire proportional chambers (MWPCs), followed by 47 absorption lengths of concrete, and then a second plane of MWPCs. Further details of this system are described in John Orthel's thesis"1.

The film was scanned for events with leaving tracks and these leaving tracks were then measured. Events with identified muons and primary vertices within the fiducial volume were measured fully. The first subsample measured consisted of a random sample of $\nu_{\mu}$ charged current events and also those charged current events in which the component of muon momentum perpendicular to the neutrino direction $\left(P_{\mu}^{T}\right)$ was greater than $4 \mathrm{GeV}$ (HIPTMU events), which gave us a sample enriched in high $Q^{2}, W^{2}$, and $E_{v}$. Most of the remaining events with identified muons have now been measured, so the starting sample for this analysis contains $7859 \nu_{\mu}$ charged cursent events, of which only 200 HIPTMU events would not have been measured otherwise.

\section{Point Measurement Errors}

The measured points of some tracks have relatively large deviations from the projections of the reconstructed trajectory onto, the film planes. This may occur for several reasons. The Coulomb scattering may be unusually large. The track may scatter hadronically through a small enough angle 
- that it is not visible to the measurer. A hadron may decay in fight with an invisible kink, but with enough change in momentum to yield a poor fit. The measurer may measure through a visible kink by mistake, or flip tracks in moving from one view to another. The camera or chamber optics may be bad for part of the fiducial volume, or the liquid may be particularly turbulent. Although none of these effects would necessarily create a spurious signal in the analysis which follows, prudence dictates that one attempt to minimize all potential problems.

The residual error on film (FRMS) is defined as twice the square root of the chi-squared per degree of freedom of the measured points relative to the projections of the reconstructed trajectory onto the film planes. For fast tracks and short tracks, the dominant contributions to FRMS usually come from the setting errors of the measuring machines and imperfect optical constants. For long slow tracks, real point scatter in space due to Coulomb interactions becomes the dominant source of FRMS. The expected contribution from this source is called CRMS. Systematic variations of residuals with depth in the bubble chamber are folded into the errors used in computing the chisquared. The quantity $F R M S^{2}-C_{R M S}^{2}$ estimates the non-Coulomb contribution to point scatter. The highest such value for a primary charged track in an event is called WORST, and it describes the quality of the tracks' reconstruction. The distribution of FRMS ${ }^{2}-C_{R M S}{ }^{2}$ of primary charged hadrons in events which nominally pass geometry is shown in figure III-1. The WORST distribution for these events is shown in figure III-2.

These distributions peak suitably low, but also have tails which clearly do not originate in statistical fiuctuations of the setting error or Coulomb scattering. A single high FRMS track may be symptomatic of a problem with the whole event measurement, such as flipping tracks in moving from one view to another. Thus we will discard events with large values of WORST rather than try to deal with the individual high FRMS tracks. The WORST distribution peaks near 3 and drops quickly. Values of WORST less than 20 certainly do not indicate bad measurements. In the analysis which follows the results for events with WORST less than 20 and the results for events with WORST between 20 and 50 are entirely consistent, while events with higher values of WORST begin to look different. Thus, only events with WORST less than 50 will be considered from here 
on.

\section{Selection of Tracks Used in Eyent Reconstruction}

In order to reconstruct the hadron system and study its properties, individual events are corrected for tracks with large momentum errors, for nuclear debris, and for particles which are not detected. Charged primary tracks, pointing $\gamma$ 's, and pointing $V^{\text {'s }}$ are accepted if they have fractional momentum errors $<0.30$ (90\% of the tracks), while all other particles are treated as undetected. Little information can be gained from including tracks with large fractional errors $(d p / p>1.0)$, and there is a trade-off in using tracks with moderate fractional errors. To the extent that a track's momentum points in the same direction as the whole hadron system, the energy reconstruction method discussed below correctly determines kinematic quantities such as $Q^{2}, W^{2}$, and $E_{p}$, which depend only on knowing the neutrino and muon momenta. Of course, almost no tracks' momenta lie even within errors of the total hadron direction, and one therefore should retain as many tracks as possible. Additionally, one must consider the effect of symmetric errors on steeply falling distributions of such quantities as transverse momentum. Mismeasuring real low $P_{T}$ tracks may feed into a high $P_{T}$ signal, but the converse will be less true as the absolute number of real high $P_{T}$ tracks is relatively small. The net result will be to create a spurious signal at high $P_{T}$. The tails of such distributions are of special interest in looking for hard gluon bremsstrahlung effects, and for this reason it is best to err on the side of rejecting more than the ideal number of tracks in the present analysis. The distribution of $d p / p$ for primary charged hadrons is shown in figure III-3. Most of the tracks have very small fractional errors, so choosing a precise cut is largely a matter of taste. Above $d p / p=0.30$ the distribution is relatively flat, and moving the cut up moderately will add relatively few tracks. Moving the cut down to $d p / p=0.20$ removes $30 \%$ more tracks and produces a negligible difference in the results. We therefore accept as visible those charged tracks with $d p / p<0.30$

Cutting on fractional momentum error introduces a momentum dependent bias. The error on the sagitta of a short or high momentum track is primarily determined by the setting error of the measuring machine. For a fixed length this will produce a higher percentage error for high 
momentum, low curvature tracks than for small momentum, high curvature tracks. Because the pion interaction length is approximately constant for all momenta greater than $2 \mathrm{GeV}$, the length distribution before interaction will be approximately flat in momentum. Therefore the percentage of high momentum tracks discarded will be greater than the percentage of low momentum tracks discarded. This is illustrated in figure III -4 , where the fraction discarded by the $d p / p$ cut is plotted as a function of measured momentum. (Below $\approx 2 \mathrm{GeV}$ the larger cross section at lower momenta tends to cancel this effect.)

The target liquid in this experiment was half (atomic) neon and half (atomic) hydrogen, so most of the target nucleons were inside neon nuclei. The subject of this study is the physics of neutrino-nucleon scattering. The nuclear target introduces uncertainties into two aspects of the analysis. First, the target nucleon is not at rest in the laboratory; it has Fermi motion relative to its nucleus. This momentum is generally less than two or three hundred $\mathrm{MeV}$ in magnitude, and therefore produces effects that are relatively small compared to those being measured. The Monte Carlo described later accounts for the Fermi motion of the target nucleons, so its effects, although not eliminated, are described and understood statistically. Second, the nuclear system interacts with the hadronic part of the weak interaction final state in some manner which is not well known. These nuclear re-interactions may add hadrons which are not part of the weak interaction to the observed final state. They may also change the momenta of hadrons which were produced in the weak interaction.

Extra hadrons from nuclear re-interactions can often be identified by their values of $x_{F}\left(x_{F} \mp 2 P_{L} / W\right)$ in the $C M$ system of the hadrons produced in the weak interaction, which has velocity $\beta_{H}$ and Lorentz boost $\gamma_{H}$ in the lab. A nucleon at rest in the lab has $\mathrm{CM}$ momentum (see equation II. 14 for notation and kinematic relationships):

$$
P C M=\gamma_{H}\left(P^{i a b}-\beta_{H} E\right)=-\beta_{H} \gamma_{H} m_{N}
$$

so that

$$
x_{f}=\frac{-\beta_{H}\left(Q^{2}+W^{2}\right)}{W^{2}}
$$

which is typically somewhat less than -1 . This is in contrast to the usual $x_{F}$ distributions which 
peak at zero and have root mean square $(\mathrm{mms})$ widths $\approx 0.15$. Most extra hadrons observed are boil-off protons that have small enough momentum in the lab that equation III. 1 is approximately true, and their $x_{F}$ values are still close to -1 . In figure III-5 the $x_{F}$ distributions are plotted for identified protons, for all positive hadrons which are not identified protons (which we call pions), and for all negative hadrons (which we call pions).

The $x_{F}$ distributions for tracks which are not identified protons peak near zero with small widths. The $x_{F}$ distribution for identified protons looks very different; it peaks near -1 rather than 0 and has most of its entries below -1. These are exactly the features one expects for boil-off protons. Most hadronic interactions are soft, low momentum transfer processes, so most nuclear reinteractions will produce low momentum baryons which will identify themselves with very negative $x_{F}$. A few low momentum pions should also be produced in such processes, and we see evidence that this is the case. As one might expect from charge conservation and nuclear charge, there is a greater signal at very negative $x_{f}$ for positive pions than for negative pions. Nota Bene: protons (and kaons) which are called pions will have reconstructed $x_{F}$ values much more positive than their true $x_{F}$ values, and at very low laboratory momentum ionization allows one to distinguish pions from protons, so the charge asymmetry at very negative $x_{F}$ does describe pion production.

\section{Event Reconstraction Algoritbm}

Events are not detected or measured perfectly, so one can only estimate the true kinematic quantities which describe them. Because the incident neutrinos have a broad energy spectrum (see figure (V-1), it is necessary to estimate each event's energy from the observed final state particles. Not all of the hadron energy or momentum is detected, and this must be accounted for. The method used here here is based on balancing perpendicular momentum in the lepton plane.

Four assumptions underlie this energy correction method:

(i) the neutrino direction is known;

(ii) the muon momentum, $\vec{P}_{\mu}$ is known;

(iii) the detected hadrons' momentum, $\vec{q}_{v i s}$, is parallel to the total hadron momentum, $\vec{q}$; 
the target nucleon is at rest; hence the components of muon momentum perpendicular to the neutrino direction exactly balance those of the hadron system, and the longitudinal momentum of the muon plus that of the hadron system equals the neutrino momentum.

If assumption (iii) is true then

$$
\frac{\left|\vec{P}_{\text {Hvis }}^{T}\right|}{\left|\vec{P}_{H}^{T}\right|}=\frac{\left|\vec{P}_{\text {Hvis }}^{\|}\right|}{\left|\vec{P}_{H}^{\|}\right|},
$$

where the superscripts $T$ and || denote the components of a vector perpendicular and parallel to the neutrino direction. The subscript vis denotes visible, which refers to that part of the vector which is detected experimentally. Since $\left|\vec{P}_{H}^{T}\right|=\left|\vec{P}_{\mu}^{T}\right|$, one can compute $P_{H}$, and with this the neutrino energy:

$$
E_{\nu}\left(=\left|P_{\nu}\right|\right)=P_{\mu}^{\|}+P_{H}^{\|}=P_{\mu}^{\|}+P_{H}^{\| v i s}\left(\left|\vec{P}_{\mu}^{T}\right| /\left|\vec{P}_{\text {Hvis }}^{T}\right|\right)
$$

While these assumptions are not exactly true, they are generally good approximations.

The angle between a neutrino and the center of the beam direction is $R / L$ where $R$ is the radial distance of its interaction vertex from the center of the neutrino beam (assumed to coincide with the center of the bubble chamber) and $L$ is the distance between the point where the neutrino is produced and the point where it interacts. These numbers are typically I meter and 1000 meters to 1400 meters, so the uncertainty in $R / L$ due to the uncertainty in the longitudinal position of the decay producing the neutrino is $\frac{1}{1000}-\frac{1}{1400}$, about $0.3 \mathrm{mr}$. The hadron beam which decays to pro duce neutrinos is typically 1 meter in radius. At the bubble chamber, 1200 meters later, the uncertainty in $\mathrm{R} / \mathrm{L}$ resulting from this finite beam size is therefore $\frac{1}{1200}$, about $0.8 \mathrm{mr}$. This adds in quadrature with the $0.3 \mathrm{mr}$ calculated above to give a total uncertainty in the neutrino direction of $0.9 \mathrm{mr} \approx 0.05^{\circ}$. This is small compared to the angles of interest and their uncertainties, so may be neglected.

Muon momenta are relatively well measured, as muons are required to have relatively long tracks. An event is in the fiducial volume only if the potential length is greater than $70 \mathrm{~cm}$, and muon tracks do not interact in the chamber. Thus the $d p / p$ distribution for muon tracks, shown in figure III-6, has much less of a tail than does that for the charged hadron tracks, shown in figure III-3. The directions of the muon tracks are also well measured, so $\vec{P}_{\mu}^{T}$ is well known. 
- The true hadron system direction is less well measured. The major contributions to the uncertainty in the hadron system direction come from not detecting all the hadrons, from the Fermi motion of the target nucleon, and from re-interactions of the final state hadrons with the nucleus containing the target nucleon. Measurement errors for the detected tracks also contribute. The net result of these effects is shown in figure III-7, where the average angle between $\vec{q}$ and $\vec{q}_{\text {is }}$, $\Theta_{Q}$, has been determined by the Monte Cario as a function of $W$, the hadron system invariant mass. The approximate form of the correlation between $\Theta_{Q}$ and $W$ can be deduced from the observation that the Lorentz boost factor of the hadron CM relative to the laboratory frame is approximately proportional to $W$ :

$$
\begin{gathered}
\gamma_{H}=\frac{E_{H}}{W}=\frac{Q^{2}+W^{2}}{2 m_{N} W} \\
=\frac{W\left(1+Q^{2} / W^{2}\right)}{2 m_{N}} \approx \frac{W\left(1+\frac{x_{B j}}{1-x_{B j}}\right)}{2 m_{N}} .
\end{gathered}
$$

The tangent of the angle of a track with respect to $\vec{q}$ is given by the ratio of its transverse and longitudinal components. A Lorentz boost leaves transverse momenta unchanged and increases longitudinal momenta with $\gamma_{H}$, so the tangent decreases as $1 / \gamma_{H}$. Since $\theta_{Q}$ derives from fluctuations of the detected tracks' directions from $\vec{q}$, one expects its magnitude to decrease as $1 / \gamma_{H}$, or $1 / W$. From the curves in figure III-7 we see that $\theta_{Q} \approx 7^{\circ} / W$, with $W$ in $\mathrm{GeV}$. (This typical error is somewhat larger than we reported previously. To mimic some of the effects of nuclear re-interactions on determining $\dot{q}$ we have since increased the magnitude of the Fermi momentum in the Monte Carlo until the Monte Carlo curves for the variables discussed below agreed with the data.)

Many detailed assumptions go into the Monte Carlo calculations of $\theta_{Q}$, from the transverse momenta of ideal tracks, to the probability of detecting the neutral particles. None of these assumptions is exactly correct, so one should check for consistency with the data. The most direct method for doing so here is to look at the projection of $\theta_{Q}$ out of the lepton plane, $\theta_{\text {aut }}$, as this can be determined for real events as well as for Monte Carlo events. The distribution of $\Theta_{\alpha u T}$ for the data is plotted in figure III-8, with the Monte Carlo curve superposed. Similarly, $\left\langle\Theta_{\text {aut }}\right\rangle$ as a function of $W$ is shown in figure III-9. Since the Monte Carlo describes this data so well one may conclude 
that it describes $\Theta_{Q}$ adequately. Perhaps surprisingly, $\left\langle\Theta_{O U T}\right\rangle \approx\left\langle\Theta_{Q}\right\rangle$, see figures III-7 and III-9. Were the target nucleon at rest in the lab, one would expect $\left.\left\langle\theta_{\text {oUT }}\right\rangle=(1 / 2)^{1 / 2}<\theta_{Q}\right\rangle$. However, in our experiment the Fermi momentum of the target nucieon is comparable to the undetected transverse momentum of the hadron system, so we measure $\theta_{Q}$ almost as well as we do $\left.\Theta_{\text {OUT. }}\right)$

Assumption (iv) underlying the energy correction method is that energy and momentum are conserved according to:

$$
\begin{gathered}
E_{\nu}+m_{N}=E_{\mu}+E_{H} \\
\vec{P}_{\nu}=\vec{P}_{\mu}+\vec{P}_{H} .
\end{gathered}
$$

However, the target nucleon is not at rest, due to Fermi motion within the neon nucleus, so the conservation equations as written are violated. The Fermi momentum of a target nucleon is typically less than $250 \mathrm{MeV}$, which is small compared to $\left|\vec{P}_{\mu}\right|$ and $\left|\vec{P}_{H}\right|$. It is not so small when compared to $\left|\vec{P}_{\mu}^{T}\right|$ or $\left|\vec{P}_{\text {Hvis }}^{T}\right|$, and this is where it has its greatest effect. The perpendicular momentum that Fermi motion adds to $\bar{P}_{H}^{T}$ is effectively subtracted from $\vec{P}_{\mu}^{T}$, which changes the perpendicular momentum balance equation. The effects of Fermi motion are smaller than one might guess from comparing the magnitude of Fermi momentum directly with $\left|\vec{P}_{\mu}^{T}\right|$. First, only the component of Fermi motion in the plane of the weak scattering enters into perpendicular momentum balance. Then, it is only the target quark momentum $\left(\xi P_{N}\right)$, not the nucleon momentum $\left(P_{N}\right)$, which affects the muon momentum; the diquark carries most of the nucleon's momentum and does not participate directly at the weak interaction vertex.

Typically, the muon perpendicular momentum in an event is $2.7 \mathrm{GeV}$. A $200 \mathrm{MeV}$ nucleon will, on average, have $200 / \sqrt{3} \mathrm{MeV}$ perpendicular momentum in the lepton plane. The apparent non-conservation of perpendicular momentum will be $\approx 115 \mathrm{MeV}$, which is about $4 \%$ of the conserved amount. This contributes directly to the error in the reconstructed neutrino energy, weighted by the ratio of hadron longitudinal momentum to neutrino longitudinal momentum. Some other kinematic quantities are affected more strongly since the error in the muon angle is propagated through the formulas a second time. 
As an example one can consider the determination of $Q^{2}$ :

$$
Q^{2}=2 E_{\mu} E_{\nu}\left(1-\cos \theta_{\mu}\right)
$$

for the event drawn schematically in figure III-10. The vectors labeled $\mu$ and $H$ show the muon and hadron momenta for an interaction where the target nucleon is at rest. The vectors labeled $\mu^{\prime}$ and $H^{\prime}$ show the same interaction where the target nucleon is moving with the indicated perpendicular momentum. Clearly $\left|\vec{P}_{\mu^{\prime}}^{T}\right|<\left|\vec{P}_{\mu}^{T}\right|$ and $\left|\vec{P}_{H^{\prime}}^{T}\right|>\left|\vec{P}_{H}^{T}\right|$, so, assuming $\left|\vec{P}_{H^{\prime} v i s}^{T}\right|>\left|\vec{P}_{H v i s}^{T}\right|$, the neutrino energy will be underestimated. The angle between the muon and the neutrino direction will also be under estimated, so $Q^{2}$ will be underestimated twice: once because $E$, is underestimated and once because $\left(1-\cos \theta_{\mu}\right)$ will be underestimated.

\section{Kinematic Reconstruction Errors}

The resolution function which describes how well a kinematic quantity $X$ is reconstructed is defined as the distribution of $\left(X_{R E C O N S T R U C T E D}-X_{T R U E}\right) / X_{T R U E}$. Such distributions are easily calculated using the Monte Carlo; the resolution functions for $E_{\eta}, E_{H}, Q^{2}, W$, and $\gamma_{H}$ are plotted in figures III-11 through III-15. The mean values and rms widths of these distributions are collected in Table III-1.

The tracks which constitute an event are often described by variables which depend not only on their individual laboratory momenta, but also on the whole event's kinematics. Feynman $x\left(x_{F}\right)$, which describes a particle's longitudinal momentum in the hadron $\mathrm{CM}$ system, and transverse momentum $\left(P_{\Gamma}\right)$ are two such variables which play important roles in the analysis which follows. The determinations of a track's $P_{T}$ and $x_{F}$ both depend on the measurement of $\hat{q}$, and that of $x_{F}$ also depends on the measurement of $\gamma_{H}$. (The measurement of $x_{F}$ also depends on the energy of the particle, which depends on its mass. Protons and kaons mis-identified as pions will be assigned $x_{F}$ values greater than if they were correctly identified.) The visible hadron direction, $\hat{q}_{\text {vis }}$, tends to be closer to the observed hadrons' directions than is $\hat{q}$, and the measured value of $\gamma_{H}$ tends to be a tad high (see Table III-1). While individual track mis-measurements tend to smear the distributions we will study without biasing them, mismeasurements of the hadron system variables tend to bias the distributions in addition to smearing them. The net result of instrumental effects on our 
measurements will be discussed as the data is presented. 
Chapter IV: Monte Cario Calculations of the Models

In order to compare the data with various models, a Monte Carlo computer program simulates neutrino-nucleon interactions and detector response. Individual events are generated according to the models described in Chapter II and then are analyzed in the same manner as are real events. By varying the parameters of the models we have studied the correlations of these parameters with observable quantities. Given a fixed model, the Monte Carlo properly convolutes the smearing and biasing effects of the detector and analysis procedures which were described in Chapter III. To the extent that these effects are model independent, the data can be corrected unambiguously and real effects established. To the extent that these effects are model dependent, one may at least determine whether the data is consistent with a specific model.

\section{Generating the Weak Interaction}

The first stage of event generation consists of picking an event's lepton and parton variables. The beam spectrum from which neutrino energies are chosen is shown in figure IV-1. This was calculated by $\mathrm{G}$. Taylor ${ }^{12}$ for the $200 \mathrm{GeV}$ tune of the Quadrupole. Triplet focusing train and includes the correlation between an event vertex's radial position in the bubble chamber and its energy. The parton momentum density functions, $\xi q(\xi)$, taken from Field and Feynman ${ }^{13}$, are shown in figure IV-2. For the weak production of charm from strange and down quarks (and of $\bar{s}$ from $\bar{u}$ ), the Cabibbo angle is set so that $\sin ^{2} \theta_{C}=0.05$. A slow rescaling and excitation function is used to account for the mass of the charmed quark ${ }^{14}$.

The additional variables required to describe three-jet $\left(W \neq q \rightarrow q g\right.$ ) events are $x_{p}, z_{q}$, and $\phi_{q}$, which were defined in equations II-16 and II-27. Because the strong coupling constant, $\alpha_{5}$, varies with an event's momentum scale, as does the Sudakov form factor, the $W^{ \pm} q \rightarrow q g$ cross section varies slowly with $E_{p}, \xi$, and $y$. This cross section is not analytically integrable, so the Monte Carlo picks $x_{p}, z_{q}$, and $\phi_{q}$ by an approximate method which numerically calculates an integral $\left(x_{p}, z_{q}\right)$ distribution only once.

$E_{m} \xi$, and $y$ are fixed at $E_{0}=750 \mathrm{GeV}, \xi_{0}=0.33$, and $y_{0}=0.6$. The differential cross sec- 
tion $\frac{d^{2} \sigma}{d x_{p} d z_{q}}$ (integrated over $\left.\phi_{q}\right)$ is then calculated for $\left(x_{p}, z_{q}\right)$ cells 0.01 by 0.01 in size, for $x_{p}$ and $z_{q}$ each varying from 0.00 to 0.99 . The integral over all these cells is stored as $d \sigma_{q g 0}$. At $\left(E_{y}, \xi, y\right)=\left(E_{0}, \xi_{0}, y_{0}\right), d \sigma_{q g 0}$ is the fraction of events which are three-jet ( $W^{ \pm} q-q g$ ), as opposed to two-jet. The ratio of the cross section calculated for one cell, times its area, to $d \sigma_{q g 0}$ is the probability that a three-jet event will have $\left(x_{p}, z_{q}\right)$ in the cell's range. The program generates a look-up table which it uses to pick $\left(x_{p}, z_{q}\right)$ with this relative probability.

For each event the Monte Carlo picks $E_{y}, \xi$, and $y$, and then generates $x_{p}$ and $z_{q}$ using the relative probability table. Because the total three-jet fraction varies slowly with the momentum scale, the Monte Carlo uses

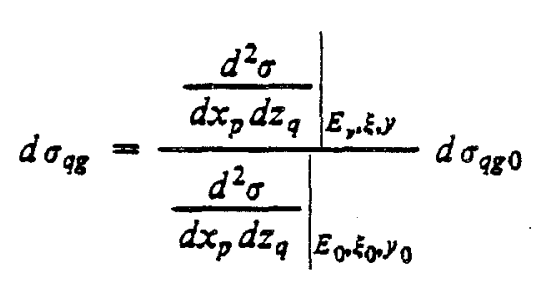

as the total three-jet cross section at $\left(E_{y}, \xi, y\right)$. A random number between 0 and 1 is generated and compared with $d \sigma_{q g}$. If the random number is less than $d \sigma_{q g}$ then the event is taken to be a three-jet event with $x_{p}$ and $z_{q}$ as chosen. Otherwise the event is taken to be a two-jet event with $x_{p}$ and $z_{q}$ each reset to 1 . The procedure by which the program produces hadron jets from partons gives poor, results for soft gluons, those which are either low energy or collinear with the final quark. Thus the program additionally requires that the quark-gluon and diquark-gluon invariant masses be at least $2 \mathrm{GeV}$; otherwise the event is taken to be two-jet and with $x_{p}$ and $z_{q}$ each reset to 1 .

For the three-jet events it remains to choose a quark azimuthal angle, $\phi_{q}$. The differential cross section has the form (see equation II.27)

$$
d \sigma_{q}=\left(A+B \cos \phi_{q}+C \cos 2 \phi_{q}\right) d \phi_{q},
$$

which when integrated over $\phi_{q}$ yields $2 \pi A$. Having decreed a three-jet event at this point, $A, B$, and $C$ (taken from equations II.28 - II.30) are simply renormalized by $1 / 2 \pi A$ and $\phi_{q}$ is chosen using the equation above to define a probability distribution.

The $x_{p}, z_{q}$, and $\phi_{q}$ distributions of the three-jet events produced by the Monte Carlo are not those one would calculate directly from the differential cross sections presented in Chapter II 
(equations II.13 - II.16) with a fixed value of $\alpha_{s}$. Both the Sudakov form factor and the absolute invariant mass cut-off are biased (see appendix B). Compared to what one would calculate directly, there are fewer three-jet events, but those generated tend to be harder. They have lower $x_{p}$ and $z_{q}$, greater quark-gluon invariant mass and parton transverse momentum. The $\left(x_{p}, z_{q}\right)$ distribution for the three-jet events produced by the Monte Cario, and its projections, are shown in figure IV-3. Figure IV-4 shows the resultant quark-gluon invariant mass distribution and figure IV-5 the gluon transverse momentum distribution.

The $\phi_{q}$ distribution in the Monte Carlo is strongly affected by the harder $\left(x_{p}, z_{q}\right)$ distribution. Both $d \sigma_{q}^{(1)}$ and $d \sigma_{q}^{(2)}$, which are the coefficients of the $\cos \phi_{q}$ and the $\cos 2 \phi_{q}$ asymmetries, take on their maximum values as $x_{p}$ and $z_{q} \rightarrow 1$, as is seen on inspection of equation II.31. The three-jet events in the Monte Cario, having a harder $\left(x_{p}, z_{q}\right)$ distribution than would be expected without the Sudakov form factor and the quark-gluon invariant mass cut-off. (i.e., smaller $x_{p}$ and $z_{q}$, thus greater $M_{q g}$ ) have less azimuthal asymmetry than do those in a direct analytic calculation. The $\phi_{q}$ distribution for the Monte Carlo three-jet events is shown in figure IV-6. The distributions of the ratios $d \sigma_{q}^{(1)} / d \sigma_{q}^{(0)}$ and $d \sigma_{q}^{(2)} / d \sigma_{q}^{(0)}(B / A$ and $C / A$ from equation IV.2) are shown in figures IV-7 and IV-8.

The average value of $d \sigma_{q}^{(1)} / d \sigma_{q}^{(0)}$ in figure IV-7 is -0.23 and the average value of $d \sigma_{q}^{(2)} / d \sigma_{q}^{(0)}$ in figure IV-8 is 0.015 . The $\cos \phi_{q}$ asymmetry looks large enough to produce observable effects. Several factors work against this. Perturbative $Q C D$ predicts this asymmetry for $W^{\neq} q \rightarrow q g$ events, but even at high $W^{2}$, most events are two-jet. Of equal importance, the $\cos \phi$ asymmetry for quarks is balanced by an opposite asymmetry for givons: when a quark is produced at $+\phi$, a gluon is produced at $-\phi$. To the extent that gluon jets look like quark jets, these cos $\phi$ asymmetries cancel. Quark and giuon jets should differ, as discussed in chapter II, section 7, but their characteristics are predicted to be sufficiently similar that the hadron $\cos \phi$ asymmetry should be an order of magnitude smaller than the parton $\cos \phi$ asymmetry. There is also a non-perturbative $\cos \phi$ asymmetry of the same sign coming from the parton model, as calculated by Cahn ${ }^{15}$ and others, which makes the interpretation of any observed signal more difficult. Experimentally, the resolution in $\phi$ is adequate 
for studies of azimuthal asymmetries only when the angle between $\vec{q}$ and $\vec{q}_{v i s}$ is small and the angle between a track's direction and $\vec{q}$ is large. This is not the case for most of our data. A full analysis of azimuthal asymmetries is beyond the scope of this thesis; preliminary results of such an analysis have been reported ${ }^{16}$ and work on the subject is continuing.

\section{Generating Hadrons From Partons}

The second stage of event generation consists of turning the final state partons into hadron jets. This is done in TUBES ${ }^{6}$, which is an extension of Field and Feynman's quark jet model ${ }^{\delta}$. TUBES generates pairs of Field and Feynman jets which it fuses together in a manner such that energy, momentum, and flavor are conserved. In QPM events the pair consists of a diquark jet and a quark jet. In $W^{ \pm} q \rightarrow q g$ events the diquark and quark are paired up with the quark and antiquark, respectively, from gluon dissociation. Because the quark and antiquark from gluon dissociation start with parallel momenta the final hadron system has a three-jet structure.

\section{The Field and Feynman Single Quark Jet Model}

The Field and Feynman model is best described by its authors:

We assume that quark jets can be analyzed on the basis of a recursive principle. The ansatz is based on the idea that a quark of type "a" coming out at some momentum $W_{0}$ in the $z$ direction creates a color field in which new quark-antiquark pairs are produced. Quark " $a$ " then

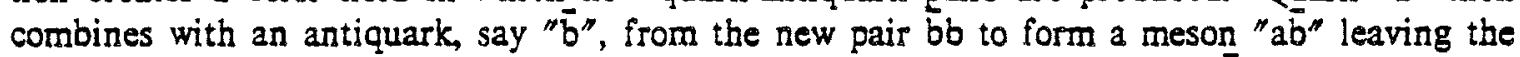
remaining quark " $b$ " to sombine with further antiquarks. The "meson" a $\bar{b}$ may be directly observed as a pseudo-scalar meson, or it may be a vector or higher-spin unstable resonance which subsequently decays into the observed mesons. To avoid complicating the ideas, we will cail "a $\bar{b}$ " the "primary" meson state and shall discuss secondary decay processes later. A "hierarchy" of primary mesons is formed of which "a $\bar{b}$ " is first in rank, "b $\vec{c}^{\prime}$ is second in rank, "cd" is third in rank, etc., as shown in fig. 1[figure IV-9]. (The "rank" in "hierarchy" should not be confused with order in momentum, but only order in the flavor relationships. The rank-2 primary meson may sometimes obtain a larger momentum than the rank-1 primary meson.)

The "chain decay" ansatz assumes that, if the rank-1 primary meson carries away a momentum $\xi_{1}$ (from a quark jet of type "a" and momentum $W_{0}$ ) the remaining cascade starts with a quark of type "b" with momentum $W_{1}=W_{0} \cdot \xi_{1}$ and the remaining hadrons are distributed in exactly the same way as the hadrons which come from a jet originated by a quark of type " $b$ " with momentum $W_{1}$. It is further assumed that for very bigh momenta, all distributions scale so that they depend only on ratios of the hadron momenta to the quark momenta. Given these assumptions, complete knowledge of the structure of a quark jet is determined by one unknown function $f(\eta)$ and three parameters describing flavor, primary meson spin, and transverse momentum ... 
In this model quark-antiquark pairs bubble up from the vacuum as $u \bar{u}$, $d \bar{d}$ and $s \bar{s}$ in the ratios 2:2:1. Half the primary mesons are chosen to be pseudo-scalar mesons and half are chosen to be vector mesons. Transverse momentum is incorporated into the model by assuming that each $q \bar{q}$ pair has no net transverse momentum, and that the magnitude of the relative transverse momentum within a pair comes from a Gaussian distribution

$$
\exp \left(-q_{T}^{2} / 2 \sigma_{q}^{2}\right) d q_{T}^{2}
$$

where $q_{T}$ is the magnitude of the quark (and antiquark) transverse momentum with respect to the jet direction. The transverse momentum, $k_{\tau}$, of a primary meson is the vector sum of the transverse momenta of the quark and the antiquark which form it. Half the primary mesons are vector mesons which decay, so the observed final state mesons have a $k_{T}$ distribution which is softer than that for the primary mesons. Field and Feynman take $\sigma_{q}=350 \mathrm{MeV}$, which gives $\left\langle k_{T}\right\rangle_{\text {primary mesons }}=439$ $\mathrm{MeV}$ and $\left\langle k_{T}\right\rangle_{\pi=}=323 \mathrm{MeV}$.

The Field and Feynman model generates hadron jets from single quarks. Our parton final states consist either of a quark and a diquark or of a quark, a gluon, and a diquark. Chris Day's extension of the Field and Feynman model, TUBES, generates hadron jets from such multi-parton final states. The original TUBES is discussed in the next section and our modifications to TUBES are discussed in the following section.

\section{Chris Day's TUBES Model}

TUBES uses the Field and Feynman model to generate single quark and diquark jets. A diquark is treated as a simple color bar state (completely analogous to an antiquark) when initiating a jet. It joins with the first quark bubbled up from the vacuum to form a baryon, which leaves an antiquark to generate the rest of the jet. Baryon species are chosen for each net fiavor state using SU(3) symmetric mixing.

Two-jet events are created by producing back-to-back quark and antiquark jets in the hadron $\mathrm{CM}$ system and tying them off. To conserve flavor and transverse momentum when fusing two jets, TUBES keeps track of the quark-antiquark pairs which go into the primary mesons and creates a 
central meson from the quark and antiquark which remain unused when the single jets are cut off.

Where to cut a single jet and stop producing hadrons requires a somewhat arbitrary algorithm. Field and Feynman take as the argument of their recursion function the ratio of $E+P_{L}$ for the hadron to $E+P_{L}$ for the initial quark, which they call $z$. This ratio is Lorentz invariant, while the comparable ratios for $E$ and $P_{L}$ separately are not. Primary hadrons are generated until one of them has $z<0$. Then all are allowed to decay, and the final hadrons with $z>0$ are taken to be part of the jet, even when $z$ of the parent hadron is less than zero.

TUBES follows this procedure until the step where primary hadrons are allowed to decay. Here it takes the primary hadron with $z<0$ and discards it. The quark (antiquark) in that meson left over from the $q \bar{q}$ pair which last contributed to a $z>0$ primary meson is tagged for later use. When two single jets have been generated they can be fused by forming a central meson from one's left over quark and the other's left over antiquark. One might argue that in order to mimic the Field and Feynman scheme the last $z>0$ meson should also be discarded and the central meson formed from the quark and antiquark which are one rank earlier in their respective jets. This, however, makes one central meson rather than the original two $z>0$ mesons (one in each of the single jets), so randomly discarding half the last $z>0$ mesons should reproduce the Field and Feynman single jet distributions. TUBES was originally written with such an algorithm. However, as discussed below, for this study two-jet events are generated by forming the central meson from the left over quarks and antiquarks from the single jets without discarding any $z>0$ primary mesons.

TUBES takes three-parton states from $W=q \rightarrow q g$ events and creates hadron jets after breaking the gluon into a quark-antiquark pair, each parton taking half the gluon's momentum. The pair's flavor is chosen as if it were bubbled up from the vacuum. The program then forms one two-jet system from the original diquark and the gluon's quark, and another from the gluon's antiquark and the original quark. The three-jet structure of the parton system (quark, diquark, and gluon) is repro duced in the hadron system because the gluon's quark and antiquark have parallel momenta in the direction of the original gluon. This is most obvious in the case where each two-jet sub-system consists of exactly two badrons. In the high energy limit, where masses can be ignored, these hadrons 
have the same momenta as their parent partons; one has the momentum of the original quark, one the momentum of the original diquark, and the other two, together, the momentum of the gluon.

\section{TUBES and Phase-Space}

The method by which TUBES generates single jets and fuses them into two-jet systems produces non-scaling phenomena. This is particularly true at the ends of the single jets where low momentum hadrons are produced. Here energy and longitudinal momenta differ substantially. As the fragmentation functions were chosen to scale in $z$, they do not scale exactly in energy or longitudinal momentum. Further, for a fixed value of $E+P_{L}$ there is limited phase space with $P_{L}>0$. Hadrons with large transverse mass, $m_{T}=\left(m^{2}+k_{T}^{2}\right)^{1 / 2}$, are more likely to fall off the end of a jet (have $P_{L}<0$ ) for a given value of $z$ than are those with small transverse mass. The result is that light mesons with small $k_{T}$ dominate jets at low $z$ (although the central meson is produced from the standard TUBES species and transverse momentum distributions, which tends to ameliorate these effects). The longitudinal momentum range where transverse mass effects become important depends only weakly on the jet energy, so where $z$ is low, in the sense considered here, depends inversely on the jet energy.

The masses of the primary mesons produced in energetic jets range from $135 \mathrm{MeV}$ for the $\pi^{0}$ to $1020 \mathrm{MeV}$ for the $\phi$. With typical transverse momenta on the order of $400 \mathrm{MeV}$, the primary mesons have transverse masses typically in the $425 \mathrm{MeV}$ to $1 \mathrm{GeV}$ range. Thus, phase-space limitations become important as jet energies approach $1 \mathrm{GeV}$. For events with small $W^{2}$, each single jet will have low energy in the hadron CM system, so the whole hadronic structure will be affected strongly by phase-space limitations. In particular, the transverse momenta of the observable hadrons will be lower for low $W^{2}$ events than for high $W^{2}$ events. At low $W^{2}$ a larger fraction of the jets' hadrons are produced at low energy, where these phase-space effects are most important. This is clearly seen in figure IV-10 where $\left\langle P_{T}^{2}\right\rangle$ is plotted as a function of $W^{2}$ for the observable hadrons in the TUBES model (using the standard values of its parameters). The effect is more pronounced for vector mesons than for pseudo-scalar mesons as the former tend to be more massive than the latter. The vector mesons decay into observable hadrons which tend to have even less transverse 
momentum than their parents, so the primary vector mesons that don't fall off the ends of the jets generated in the low $W^{2}$ events tend to produce lower transverse momentum observable hadrons than do the primary pseudo-scalar mesons. The suppression of $P_{T}$ at low $W^{2}$ is stronger in the standard TUBES model than in the data, so we set the fraction of primary mesons produced as vector mesons to rise linearly from 0 to $\frac{1}{2}$ between $W=2$ and $10 \mathrm{GeV}$. We also increased $\sigma_{q}$ from Field and Feynman's $350 \mathrm{MeV}$ to $380 \mathrm{MeV}$, which raises $\left\langle P_{T}^{2}\right\rangle$ at all $W^{2}$. With these modifications, the QPM and $Q C D$ models both describe our $\left\langle P_{T}^{2}>\right.$ data for $W^{2}<50 \mathrm{GeV}^{2}$.

While $\left\langle P_{T}^{2}\right\rangle$ depends only on the second moment of the $k_{T}$ distribution, other variables are more sensitive to the actual shape of the $k_{T}$ distribution. We found that adding a larger high $k_{T}$ tail to the distribution, while hoiding the rms width constant, generally improved the Monte Carlo's description of the data. We therefore replaced the gaussian $q_{T}$ distribution with a Student's tdistribution which has the same rms width. (Student's t-distribution is defined as:

$$
S_{n}(x)=K\left[1+\frac{x^{2}}{n} !^{-(n+1) / 2}\right.
$$

where $K$ is a normalization constant. We used $n=10$, which gives a shape somewhere between that of a gaussian and that of a Breit-Wigner.) 


\section{Chapter V: Analyzing the Data}

QCD predicts that in hard gluon bremsstrahlung events $\left(W^{ \pm} q \rightarrow q g\right.$ ) the quarks and giuons are predominantly emitted in the forward direction of the hadron CM system while the diquark is emitted in the backward direction. Consequently, the transverse momentum of forward particles should be greater than that of backward particles. This difference in transverse momentum is found only in the plane formed by the three partons and not out of that plane. As parton (quark and giuon) transverse momentum increases with $W^{2}$, so does the transverse momentum of forward hadrons. Thus, one may investigate the transverse momentum properties of the hadrons produced in deep inelastic scattering events for evidence of hard gluon bremsstrahlung.

\section{QCD Predictions for Quark and Gluon Transverse Momentum}

In the hadron $C M$ system the polar angle of a final state parton, $\cos \theta_{f}$, taken with respect to the current direction, depends only on $\xi, x_{p}$, and $z_{q}$ (see equation II.24). In figure V-1 the distributions of the quark and gluon polar angles for Monte Carlo three-jet events are shown. The giuons are generally found at wider angles than the quarks as $z_{\mathrm{g}}$ is generally less than $z_{q}$ (recall from Chapter II, section 5 that $z_{q}+z_{g} \approx 1$ and see figure IV-3(c)). and the quark and gluon transverse momenta are of equal magnitude. Both quarks and gluons are predominantly emitted forward in the hadron $\mathrm{CM}$ system, ie., $\cos \theta_{f}>0$. The quark and the gluon together must be forward; to have one forward and the other backward demands that the quark-gluon invariant mass be relatively high (a low $x_{p}$ event) and that the quark-gluon relative momentum $\left(\vec{P}_{q}-\vec{P}_{g}\right)$ in the quark/gluon CM system be mostly along the current direction $\left(z_{g} \ll z_{q}\right.$ for $\theta_{g}<0$ or $z_{q} \ll z_{g}$ for $\left.\theta_{q}<0\right)$. (The $\left(x_{p}, z_{q}\right)$ scatter plot is shown in figure IV-3.)

The transverse momentum of quarks and gluons emitted in $W^{ \pm} q \rightarrow q g$ events will increase with $W^{2}$. For fixed $\xi, x_{p}$, and $z_{q}$ the parton transverse momentum, $P_{T P}$, is proportional to $W$, as $\xi$, $x_{p}$, and $z_{q}$ fix the parton geometry in the hadron CM system (see equations II.22 - II.24) and $W$ is the energy scale there. The $W^{ \pm} q \rightarrow q g$ cross-section (II.27 - II.30) approximately scales with $W^{2}$, so $P T_{T P}^{2}$ approximately scales with $W^{2}$. In the calculation of $\left\langle P \frac{2}{T P}\right\rangle$ as a function of $W$, shown in figure V-2, several factors do not scale exactly. The coupling constant, $\alpha_{s}$, decreases with increasing 
$M_{q g}^{2}$. Hence, any particular three-jet configuration is less likely at higher $W^{2}$ than at lower $W^{2}$ (relative to $\frac{d^{2} \sigma}{d x d y}$ at the relevant energy). This effect tends to keep $\left\langle P \frac{2}{T P}>\right.$ from increasing as fast as $W^{2}$. On the other hand, since the Monte Carlo uses an absolute $M_{q g}^{2}$ cut-off to define hard giuon bremsstrahlung, high $W^{2}$ events with small angle partons will be kept as three-jet events where low $W^{2}$ events with the same parton geometry will have $M_{q 8}^{2}$ less than the cut-off value, and therefore be taken as two-jet events with $x_{p}$ and $z_{q}$ each reset to 1 . The fraction of three-jet events therefore increases at high $W^{2}$, pushing $\left\langle P_{T P}^{2}\right\rangle$ to grow faster than $W^{2}$. Most importantly, there is also a correlation between $W^{2}$ and $P \frac{2}{T P}$ which comes from the correlation of each of these variables with $\xi$ : high $W^{2}$ correlates with low $\xi$ (see equation II.14c), which in turn correlates with low $P_{T P}$ (see equation II.26). As seen in figure V-3, the variation of $\xi$ with $W$ is particularly strong for three-jet events.

\section{QCD Predictions for Hadron Transverse Momentum}

In this experiment, as in all others, hadron momenta are measured, not parton momenta. If one assumes that partons evolve into jets of hadrons with each jet's momentum (approximately) equal' to its parent parton's momentum, then a parton's transverse momentum (with respect to the current direction), $P_{T P}$, will be partitioned amongst the hadrons of its jet. Thus, the QCD predictions for parton transverse momentum are roughly translated into predictions for hadron transverse momentum.

To the extent that each hadron in a jet moves exactly in the jet direction, its momentum is a fraction $\left(z_{h}^{g(g)}\right)$ of the parent parton's momentum:

$$
\vec{P}_{h}=z_{h}^{g(g)} \cdot \vec{P}_{q(g)},
$$

and this describes both its longitudinal and transverse components. We see that $z_{h}^{q(g)}$ introduces a correlation between a hadron's longitudinal momentum and its transverse momentum which tends to give higher $x_{F}$ hadrons higher transverse momentum than lower $x_{F}$ hadrons. However, the quark and gluon start with transverse momenta of equal magnitude, and at least one of them has $z_{f}<0.5$. The hadrons in the jet from a lower $z_{f}$ parton tend to be produced at lower $x_{F}$ than the hadrons in 
the jet from a higher $z_{f}$ parton, so high transverse momentum hadrons are also produced at low $x_{F}$. Because $P_{T P}$ is proportional to $z_{q(g)} \cdot\left(1-z_{q(g)}\right)$ (see equation II.26) hadrons evolving from low or high $z_{f}$ partons have smaller transverse momentum than do those evolving from medium $z_{f}$ partons. This, too, acts to correlate a hadron's longitudinal and transverse momenta.

Processes other than hard gluon bremsstrahlung also generate correlations between transverse and longitudinal momenta within jets. To test $Q C D$, we must distinguish predictions which are unique to it from those which are common to other models. Specifically, we want to make measurements where the QCD and QPM predictions are qualitatively different.

\section{3. $\langle P \underset{T}{2}\rangle$ as a Test of $Q C D$}

High transverse momentum hadrons from hard gluon bremsstrahlung are emitted predominantly in the forward direction, and their production depends significantly on $x_{F}$, for the reasons outlined above. This is seen in figure V-4 where $\left\langle P_{T}^{2}\right\rangle$ is plotted as a function of $x_{F}$ for the two jet QPM model and again for the QCD model. The two-jet curve shows a symmetric sea-gull effect. The QCD curve shows an asymmetric sea-gull effect. The difference between the two, $\delta P_{T}^{2}$, also plotted, is due to hard gluon bremsstrahiung. Because the sea-gull effect is symmetric in the two-jet model, and because the backward direction is unaffected by the hard gluon bremsstrahlung in the QCD model, we will use the backward direction as a standard for comparison with the forward direction.

Traditionally, one measures $\left\langle P_{T}^{2}\right\rangle$ as a function of $Q^{2}$ or $W^{2}$ for, the forward hadrons, and looks for an increase one can attribute to gluon bremsstrahlung. In eariy analyses, a hadron was considered to be forward if its energy fraction in the lab, $z_{L} \mp E_{h} / \nu$, was greater than some cut-off value, typically $z_{L}>0.2$. Jet models and longitudinal phase-space models were assumed to give $\left\langle P_{T}^{2}\right\rangle$ independent of $Q^{2}$ and $W^{2}$, and therefore any increase observed was taken as evidence for QCD. There are problems with this procedure. The two-jet $Q P M$ prediction for $\left\langle p_{T} \frac{2}{T}\right\rangle$ of all charged hadrons is plotted as a function of $W$ in figure V-5. For $W^{2}<50 \mathrm{GeV}^{2}$, phase-space limitations dominate $P_{T}$, so $\left\langle P_{T}^{2}\right\rangle$ grows with $W^{2}$. Further, a fixed $z_{L}$ cut in the lab corresponds to an $x_{F}$ cut in the hadron $\mathrm{CM}$ system which varies with $W^{2}$; at higher $W^{2}$ it generally corresponds to a 
higher $x_{F}$ cut than at lower $W^{2}$, which, due to the symmetric seagull effect, also tends to increase $\left\langle P_{T}^{2}\right\rangle$ at higher $W^{2}$.

In anaiyses of forward hadrons only, the prediction that $\left\langle P_{T}^{2}\right\rangle$ should grow with $W$ is common to most models, and the observation of such an effect is not necessarily evidence for hard gluon bremsstrahlung. However, one can remove the conventional background to a real $Q C D$ signal by considering the forward-backward difference in $\left\langle P_{T}^{2}\right\rangle, \Delta P_{T}^{2} \mp\left\langle P_{T}^{2}\right\rangle_{F}-\left\langle P_{T}^{2}\right\rangle_{B}$, where the subscripts $B$ and $F$ stand for backward and forward in the hadron CM system.

Since the $Q C D$ prediction for $\delta P_{T}^{2}$ has the $x_{F}$ dependence shown in figure V-4, $\Delta P_{T}^{2}$ is maximized if one considers high $x_{F}$ tracks only. In figure $V-6$ the $Q C D$ predictions for $\left\langle P_{T}^{2}\right\rangle_{F},\left\langle P_{T}^{2}\right\rangle_{B}$, and $\Delta P_{T}^{2}$ are plotted as functions of $W^{2}$, first for all charged hadrons, then for only those with $\left|x_{F}\right|>0.05,\left|x_{F}\right|>0.10$, and $\left|x_{F}\right|>0.20$. The extra hadron transverse momentum due to hard glụon bremsstrahlung becomes more pronounced as higher $\left|x_{F}\right|$ regions are considered. We want to establish that $\Delta P_{T}^{2}$ is significantly non-zero. In our experiment, we are limited by statistics. As one raises the $\left|x_{F}\right|$ cut to increase the measured valus of $\Delta P_{T}^{2}$, the number of tracks included in the measurement decreases, and so the measured fractional uncertainty increases. The $x_{F}$ distribution peaks sharply at zero, see figure $V_{-7}$, so cutting on sufficiently high $\left|x_{F}\right|$ causes the uncertainty in $\Delta P_{T}^{2}$ to grow faster than $\Delta P_{T}^{2}$ itself. Hence, we must strike a balance between the increasing value of $\Delta P_{T}^{2}$ and the increasing error on $\Delta P_{T}^{2}$ as we raise the $x_{F}$ cut.

\section{Experimental Results for $\left\langle P_{T}^{2}\right\rangle$}

In figure V-8 the experimentally measured values of $\left\langle P_{T}^{2}\right\rangle_{F},\left\langle P_{T}^{2}\right\rangle_{B}$, and $\Delta P_{T}^{2}$ are plotted as functions of $W$ for $\left|x_{F}\right|>0.00,\left|x_{F}\right|>0.05,\left|x_{F}\right|>0.10$, and $\left|x_{F}\right|>0.20$. In each case the QPM and QCD Monte Carlo calculations, including instrumental effects, are also shown. Both the $\left\langle P_{T}^{2}\right\rangle_{B}$ and the $\left\langle P_{T}^{2}\right\rangle_{F}$ data points rise similarly with $W$ until approximately $W=7 \mathrm{GeV}$, after which $\left\langle P_{T}^{2}\right\rangle_{F}$ grows faster than does $\left\langle P_{T}^{2}\right\rangle_{B}$. The difference, $\Delta P_{T}^{2}$, starts out somewhat less than zero, and above $W=10 \mathrm{GeV}$ rises to values significantly greater than zero. As the $\left|x_{F}\right|$ cut is raised, $\left\langle P_{T}^{2}\right\rangle_{B}$ and $\left\langle P_{T}^{2}\right\rangle_{F}$ rise, indicating a sea gull effect. At high $W^{2}, \Delta P_{T}^{2}$ is significantly greater than zero, and rises with the $\left|x_{F}\right|$ cut, so the sea gull is asymmetric, as expected (see also 
figure $V-33$ ).

The Monte Carlo curves in figure V-6 differ from those in figure V-8 only because instrumental effects have been included in calculating the latter. In sum, these effects produce measured $\left\langle P_{T}^{2}\right\rangle_{B}$ greater than true $\left\langle P_{T}^{2}\right\rangle_{B}$ and measured $\left\langle P_{T}^{2}\right\rangle_{F}$ less than true $\left\langle P_{T}^{2}\right\rangle_{F}$, yielding measured $\Delta P_{T}^{2}$ less than true $\Delta P_{T}^{2}$. This net bias originates in the cuts which ensure that most of the hadron energy in an event has been detected.

The above bias can be understood qualitatively as follows. In the lab, the longitudinal momenta of the forward hadrons are much greater than the longitudinal momenta of the backward hadrons. If all the forward hadrons in an event are detected, and none of the backward hadrons, the event will probably pass cuts. If all the backward hadrons are detected, and none of the forward hadrons, the event will probably not pass cuts, as most the the hadron energy will be lost. In the first case $\dot{q}_{v i s}$ is exactly the direction of the forward hadrons. In the second case $\dot{q}_{v i s}$ is exactly the direction of the backward hadrons. In general, when $\vec{q}_{v i s}$ is closer to the forward hadrons' direction than the backward hadrons' direction, a larger fraction of the total longitudinal momentum is detected, and the event is more likely to pass cuts. When $\vec{q}_{\text {vis }}$ is closer to the forward hadrons' direction than is $\dot{q}$, forward $P_{T}$ tends to be under-measured. When $\vec{q}_{v i s}$ is closer to the backward hadrons' direction than is $\dot{q}$, backward $P_{T}$ tends to be under-measured. As events with $\vec{q}_{v i s}$ closer to the forward hadrons' direction are more likely to pass cuts than are those with $\vec{q}_{v i s}$ closer to the backward hadrons' direction, the net effect is as seen in the Monte Carlo curves presented above: $\left\langle P_{T}^{2}\right\rangle_{B}$ is measured slightly high and $\left\langle P_{T}^{2}\right\rangle_{F}$ is measured low, so that in a forward-backward symmetric world $\Delta P_{T}^{2}$ is measured less than zero.

Below $W^{2}=50 \mathrm{GeV}^{2}$, the data are consistent with the QPM (and QCD) calculations, and although $\left\langle P_{T}^{2}\right\rangle_{F}$ and $\left\langle P_{T}^{2}\right\rangle_{B}$ rise significantly, $\Delta P_{T}^{2}$ does not. Above $W^{2}=50 \mathrm{GeV}^{2}$, the measured values of $\Delta P_{T}^{2}$ rise above the $Q P M$ curve, and above $W^{2}=100 \mathrm{GeV}^{2}$, the measured values of $\Delta P_{T}^{2}$ rise above zero absolutely. In the $\left|x_{F}\right|>0.05, W^{2}>50 \mathrm{GeV}^{2}$ data, where Monte Carlo studies indicate we are most sensitive to hard gluon bremsstrahlung effects, $\Delta P_{T}^{2}=0.047 \pm 0.011$, which is significantly greater than zero and greater, still, than the QPM expectation $(\approx-0.03$ ). As 
the $\mathrm{QPM}$ calculation describes $\left\langle P_{T}^{2}\right\rangle_{B}$ reasonably well, one may conclude that the $\Delta P_{T}^{2}$ data are not consistent with any forward-backward symmetric model at high $W^{2}$.

In measuring hadron $P_{T}$ we have approximated the current direction, $\dot{q}$, by the direction of the visible hadrons, $\dot{q}_{v i s}$. We estimate that the amount by which these directions differ (see figure III-7), and the effect this difference has on $\left\langle P_{T}^{2}\right\rangle$ and $\Delta P_{T}^{2}$, is not negligible. Because our estimates of instrumental effects cannot be exactly correct, we would like to make measurements of $P_{T}$ which are independent of $\vec{q}_{v i s}$. From conservation of momentum, $\vec{q}$ lies in the lepton plane. The component of a hadron's transverse momentum out of that plane, $P_{\text {out }}$, is independent of $\widetilde{q}_{\text {vis }}$. To the extent that the azimuthal distribution of hadrons about $\vec{q}$ is flat, $P_{\text {OUT }}$ is an unbiased estimator of $P_{T}$, when averages over large numbers of tracks are considered. In that case $\left\langle P_{\text {ouT }}^{2}\right\rangle=$ $\frac{1}{2}\left\langle P \frac{2}{T}\right\rangle$

In figure $V-9<P_{O U T}^{2}>_{B},\left\langle P_{O U T}^{2}>_{F}\right.$, and $\Delta P_{O U T}^{2} \equiv\left\langle P_{O U T}^{2}>_{F}-\left\langle P_{O U T}^{2}\right\rangle_{B}\right.$ are plotted as functions of $W$ for $\left|x_{F}\right|>0.05$, as are the corresponding QPM and QCD curves. The Pout data show the same structure as do the $P_{T}$ data, but with somewhat less statistical significance as $P_{\text {OuT }}$ is the convolution of $P_{T}$ with the sine of the hadron's azimuthal angle about $\vec{q}$. The ratio of $\left\langle P_{T}^{2}\right\rangle_{F(B)}$ to $\left\langle P_{O U T}^{2}\right\rangle_{F(B)}$ is plotted as a function of $W$ in figure $\mathrm{V}-10 \mathrm{a}(\mathrm{b})$ with the Monte Carlo curve superposed. The ratio is not exactly 2 and varies with $W$. The systematic errors in $\left\langle P_{\text {OUT }}^{2}\right\rangle$ differ from those in $\left\langle P_{T}^{2}\right\rangle$, so the ratio predicted by the Monte Carlo also is not exactly 2 . The visible hadron direction is not sensitive to errors in neutrino or muon direction; the lepton piane is. Also, the visibie hadron direction naturally includes the Fermi momentum of the nucleon; the lepton plane does not. Conversely, the visibie hadron direction is sensitive to undetected hadrons; clearly, the lepton plane is not. Because the systematic errors for $\left\langle P_{T}^{2}\right\rangle$ and $\left\langle P_{O U T}^{2}\right\rangle$ are different, the observation that they behave similarly gives us confidence that the $\Delta P_{T}^{2}$ signal is real.

\section{Event Transverse Miomentum}

Traditionally, one investigates transverse momentum production by studying variables, such as $\left\langle P_{T}^{2}\right\rangle$ and $\Delta P_{T}^{2}$, that describe the ensemble properties of tracks from many events. One may inves- 
tigate transverse momentum production in greater detail by studying variables that describe the transverse momentum structure of individual events. If hard gluon bremsstrahlung is responsible for the rise of $\Delta P_{T}^{2}$ with $W^{2}$, one would like to measure the transverse momentum of the quarks and gluons in three-jet events.

Georgi and Sheiman ${ }^{17}$ suggested that one could do so by summing the magnitudes of the transverse momenta of the final state hadrons:

$$
\pi_{G \& S} \equiv \Sigma\left|\vec{P}_{T}\right| \text {. }
$$

In the limit that the parton transverse momentum is much greater than the typical transverse momentum of a hadron with respect to its jet, most of an event's transverse momentum comes from the parton transverse momentum, so $\pi_{G \& S} \approx 2 P_{T P}$. In our experiment this rarely is expected to be the case, so $\pi_{\text {Ges }}$ serves, instead, to statistically separate two-jet events from three-jet events, to the extent that the former have low values of $\pi_{G \& S}$, and the latter have high values of $\pi$ G\&S. Several refinements to $\pi_{G \& S}$ allow a better separation.

According to $Q C D$, hard gluon bremsstrahlung produces hadron transverse momentum predominantly in the forward direction. Experimentally, $\left\langle P_{T}^{2}\right\rangle_{F}$ is greater than $\left\langle P_{T}^{2}\right\rangle_{B}$ at high $W^{2}$, giving credence to the QCD hypothesis. We will therefore consider the forward and backward directions separately, and look for a hard gluon bremsstrahlung signal in the forward direction.

In two-jet events each track on average has $P_{T}$ equal to the typical two-jet value, $P_{T 0}$. By subtracting $P_{T 0}$ once for each track contributing to a sum such as $\pi_{G \alpha S}$, one constructs a variable whose distribution, for a sample of two-jet events, approximates that due to a random walk starting from zero. This distribution peaks at zero and has a width which grows as the square root of the number of contributing tracks.

For a sample of two-jet events, the distribution of the variable

$$
\pi_{F(B)} \equiv \frac{A}{\sqrt{n_{F(B)}}} \sum_{F(B)}\left(\left|\vec{P}_{T}\right|-P_{T 0}\right)
$$

where the sum is taken over the $n_{F(B)}$ forward (backward) charged tracks, should, therefore, peak near zero and have a width approximately independent of contributing multiplicity. ( $\pi_{F(B)}$ is 
defined to be zero for $n_{F(B)}=0$.) As in calculating $\left\langle P_{T}^{2}\right\rangle$, one may consider only tracks with $\left|x_{F}\right|$ greater than some minimum value in calculating $\pi_{F(B)}$. The distribution of $\pi_{B}$ for hard gluon bremsstrahiung events should be the same as that for two-jet events, but the distribution of $\pi_{F}$ should be harder, with a greater tail at high values.

For the analysis which follows, we set $P_{T 0}=0.320 \mathrm{GeV}$ and $\mathrm{A}=4.0 \mathrm{GeV}^{-1}$, which gives the $\pi$ distributions peaks near zero and widths approximately unity. The $\pi_{F}$ and $\pi_{B}$ distributions for the total event sample are shown in figure V-11. All $x_{F}>0$ tracks have been included in the $\pi_{F}$ sums, and all $x_{F}<0$ tracks have been inciuded in the $\pi_{B}$ sums.

The total event sample has been divided into subsamples according to the multiplicity, $n_{F(B)}$, contributing to $\pi_{F(B)}$. The mean values and widths of the $\pi_{F(B)}$ distributions for these subsamples are collected in Table V-1. The mean value of $\pi_{F(B)}$ decreases slightly with increasing multiplicity, but the width of the $\pi_{F(B)}$ distribution shows no systematic variation with multiplicity. Thus, to first approximation, $\pi_{F(B)}$ is a normally distributed variable, independent of contributing multiplicity. High $\pi_{F}$ does not correlate with high multiplicity, as does high $\pi_{G a s}$, for instance, so it is a relatively clean signature for a hard transverse momentum process, such as hard gluon bremsstrahlung.

In the same way that parton transverse momentum from hard gluon bremsstrahlung should lead to $\left\langle P_{T}^{2}\right\rangle_{F}$ increasing with $W$, it should lead to $\left\langle\pi_{F}\right\rangle$ increasing with $W$. As there may be forward-backward symmetric rises of $\langle\pi\rangle$ due to phenomena other than hard gluon bremsstrahlung, one profits from comparing the data describing the forward direction with those describing the backward direction. In figure $\left.\left.V-12<\pi_{B}\right\rangle,<\pi_{F}\right\rangle$, and $\left.\Delta \pi \mp<\pi_{F}>-<\pi_{B}\right\rangle$ are plotted as functions of $W$ for $\left|x_{F}\right|>0.00,\left|x_{F}\right|>0.05,\left|x_{F}\right|>0.10$, and $\left|x_{F}\right|>0.20$. The corresponding QPM and QCD curves are also plotted for each set of data points. The $\left|x_{F}\right|>0.05$ cut gives $\Delta \pi$ the most significant rise with $W$, which is consistent with the $\left\langle P_{T}^{2}\right\rangle$ results and with the QCD Monte Carlo studies. From this point we will mean the $\pi$ variable for $\left|x_{F}\right|>0.05$ whenever we refer to a $\pi$ variable.

The $\left\langle\pi_{F}\right\rangle,\left\langle\pi_{B}\right\rangle$, and $\Delta \pi$ data vary with $W$ much as do the $\left\langle P_{T}^{2}\right\rangle_{F},\left\langle P_{T}\right\rangle_{B}$, and $\Delta P_{T}^{2}$ data. For $W>10 \mathrm{GeV}(7 \mathrm{GeV}), \Delta \pi=0.25 \pm 0.05(0.15 \pm 0.03)$, which is significantly greater than 
zero and greater, still, than the QPM expectation (-0.15).

Instrumental effects bias the $\pi$ measurements in the same way that they bias the $P_{T}^{2}$ measurements. Measured values of $\pi_{B}$ tend to be greater than true values of $\pi_{B}$, and measured values of $\pi_{F}$ tend to be less than true values of $\pi_{F}$. This is seen in figure V.13 where QPM and QCD calculations of the $\pi_{F}$ and $\pi_{B}$ distributions at high $W^{2}$ are presented, with instrumental effects included and excluded. The "net efficiency" ratio, $\epsilon_{F(B)}$, of $\frac{1}{N} \frac{d N}{d \pi_{F(B)}}$ for this experiment's detector to $\frac{1}{N} \frac{d N}{d \pi_{F(B)}}$ for an ideal detector is plotted in figure V-14 for both the QPM and QCD models. $\epsilon$ is model dependent because it measures the net migration of events from one range of $\pi$ to another. Consider the high $\pi_{F}$ region. The QCD high $\pi_{F}$ tail is substantially greater than the QPM high $\pi_{F}$ tail (see figure V-13a), so instrumental effects will move more events (although the same fraction) out of a QCD tail than out of a QPM tail. Instrumental effects will move similar numbers of events into the high $\pi_{F}$ tail in both models as the number of medium $\pi_{F}$ events (which are the source of the false high $\pi_{F}$ events) is roughly the same. Hence the QPM Monte Carlo gives a higher value of $\epsilon_{F}$ at high $\pi_{F}$ than does the QCD Monte Carlo. As the $\pi_{B}$ distributions of the QCD and QPM models are the same, the $\epsilon_{B}$ 's are also the same. To the extent that $\epsilon_{F(B)}$ is model independent, the observed $\pi_{F(B)}$ distributions can be translated into true $\pi_{F(B)}$ distributions. To the extent that $E$ is model dependent, the observed $\pi_{F(B)}$ distributions must be compared with model calculations that include instrumental effects.

The experimental $\pi_{F}$ and $\pi_{B}$ distributions for $W^{2}<50 \mathrm{GeV}^{2}$ and $W^{2}>50 \mathrm{GeV}^{2}$ are shown in figure V-15. For $W^{2}<50 \mathrm{GeV}^{2}$ the $\pi_{F}$ and $\pi_{B}$ distributions are very similar; however, for $W^{2}>$ $50 \mathrm{GeV}^{2}$ the forward distribution has a larger tail at high $\pi$. Instrumental effects decrease the observed fraction of high $\pi_{F}$ events more than they decrease the observed fraction of high $\pi_{B}$ events, so the true forward-backward asymmetry at high $W^{2}$ is greater than the difference of the raw numbers of high $\pi_{F}$ and high $\pi_{B}$ events.

The raw numbers of events observed for ranges of $\pi_{F}$ and $\pi_{B}>1.6$ are collected in Table $V$. 2a for the $W^{2}<50 \mathrm{GeV}^{2}$ data and the $W^{2}>50 \mathrm{GeV}^{2}$ data. In Table $\mathrm{V}-2 \mathrm{~b}$ the forward-backward 
symmetric prediction for each of the $\pi_{F}$ ranges is calculated from the observed number of events with $\pi_{B}$ in the corresponding range, the net efficiencies $\epsilon_{B}$ and $\epsilon_{F}$, and the relative numbers of events with at least one forward or backward track. The net high $\pi_{F}$ signal, over this symmetric background, is reported in Table V-2c. As an example, for $W^{2}>50 G e V^{2}$ we observe 31 events with $\pi_{B}>3.0$. The relative net efficiency for detecting a high $\pi_{F}$ event compared to detecting a high $\pi_{B}$ event is about 0.57 , and the ratio of the number of events with $n_{F}>0$ to that with $n_{B}>0$ is 1.23 . Thus, the forward-backward symmetric (two-jet) contribution to $\pi_{F}>3.0$ is $\approx 31 \times 0.57 \times 1.23 \approx 22$ events. We observe 89 , leaving a net signal of $67 \pm 11$ (statistical error oniy) more $\pi_{F}>3.0$ events than expected from conventional sources. The high $W^{2}$ results of Table V-2 are summarized in figure $\mathrm{V}-16$, where the net rate of $\pi_{F}$ events (above the rate calculated assuming forwardbackward symmetry) is plotted for medium to high $\pi_{F}$.

Because QCD predicts that hard gluon bremsstrahlung produces high transverse momentum hadrons in the forward direction mainly, we have studied the forward and backward directions separately. This distinguishes and enhances any hard gluon bremsstrahlung signal. We can also study the forward and backward directions jointly. Scatter plots of $\left(\pi_{F}, \pi_{B}\right)$ for $W^{2}<50 \mathrm{GeV}^{2}$ events and $W^{2}>50 \mathrm{GeV}^{2}$ events are shown in figures $\mathrm{V}-17 \mathrm{a}$ and $\mathrm{V}-17 \mathrm{~b}$. There is some correlation of $\pi_{B}$ and $\pi_{F}$, which is more clearly seen in figure $V-18$, where $<\pi_{B}>$ is plotted as a function of $\pi_{F}$, and in figure $\mathrm{V}-19$, where $\left\langle\pi_{F}\right\rangle$ is plotted as a function of $\pi_{B}$. The $\pi_{F}-\pi_{B}$ correlation func. tion,

$$
C_{\pi}=\frac{<\pi_{F} \pi_{B}>-<\pi_{F}><\pi_{B}>}{\left[\left(<\pi_{F}^{2}>-<\pi_{F}>^{2}\right) \cdot\left(<\pi_{B}^{2}><\pi_{B}>^{2}\right)\right]^{1 / 2}},
$$

is 0.17 for the $W^{2}<50 \mathrm{GeV}^{2}$ events and is 0.13 for the $W^{2}>50 \mathrm{GeV}^{2}$ events. The QPM calculations give $C_{x}=0.11$ for the $W^{2}<50 \mathrm{GeV}^{2}$ events and $C_{\pi}=0.05$ for the $W^{2}>50 \mathrm{GeV}^{2}$ events. The QCD calculations give $C_{\pi}=0.13$ for the $W^{2}<50 \mathrm{GeV}^{2}$ events and $C_{\pi}=0.10$ for the $W^{2}>$ $50 \mathrm{GeV}^{2}$ events. We estimate the statistical uncertainty in $C_{\pi}$ from its variation from one Monte Carlo run to another to be about 0.01 for the $Q P M$ and $Q C D$ values and about 0.03 for the data values. We also note that in the pristine Monte Carlos the $C_{x}$ values are 0.05 to 0.10 less than in the corresponding smeared Monte Carlos. As the systematic and statistical uncertainties are large 
compared to the differences between the QPM and QCD predictions, we cannot say that the data prefer one model over the other.

\section{Transverse Momentum Shape}

So far we have studied only the magnitudes of hadron transverse momenta. Because $P_{T}$ due to hard giuon bremsstrahlung should be found in the plane formed by the quark, gluon, and diquark momenta, we want also to study the extent to which hadron transverse momenta lie in such a plane. We define $P_{T 1}$ and $P_{T 2}$ as the projections of a hadron's transverse momentum on $\dot{u}_{1}$ and $\dot{u}_{2}$, the two orthogonal directions in the plane normal to $\vec{q}$ which maximize the sum

$$
\frac{\sum\left(P_{T 1}^{2}-P_{T 2}^{2}\right)}{\sum\left(P_{T 1}^{2}+P_{T 2}^{2}\right)}
$$

We then define planarity, $P$, as the maximum value of this sum. These sums are taken over all hadrons in an event (including neutrals), so that planarity measures the transverse shape of the hadron system as a whole. Planarity ranges from zero, for events whose hadron systems are round, to one, for events whose hadron systems are flat. A hadron system is round if its transverse momenta are distributed more or less equally in azimuthal angle about $\vec{q}$. A hadron system is flat if its transverse momenta are distributed in two bunches, opposite each other with respect to $\vec{q}$. In a flat hadron system $\hat{u}_{1}$ is a line connecting the two bunches of transverse momenta, and ( $\left.\dot{q}, \dot{u}_{1}\right)$ defines a hadron plane. In a round hadron system no such plane exists.

Planarity is independent of all longitudinal variables. It is independent of the overall scale of transverse momentum, and depends only on the relative magnitudes and directions of the transverse momenta in an event. Given an event with some planarity, were all the transverse momenta increased by the same multiplicative factor, the planarity would remain unchanged. Thus, planarity measures only transverse shape.

Planarity has a strong multiplicity dependence as is seen in figure V-20, where the average value of planarity is plotted as a function of charge multiplicity for the $W^{2}<50 \mathrm{GeV}^{2}$ and the $W^{2}$ $>50 \mathrm{GeV}^{2}$ samples. The average value of planarity decreases with increasing multiplicity and shows no $W^{2}$ dependence for any given multiplicity. Average multiplicity increases with $W^{2}$, see 
figure V-21, so the planarity distribution for the $W^{2}<50 \mathrm{GeV}^{2}$ sample has a mean value, $\langle P\rangle=0.55 \pm 0.01$, somewhat greater than that of the $W^{2}>50 \mathrm{GeV}^{2}$ sample, $\langle P\rangle=0.51 \pm 0.01$. These planarity distributions are shown in figure V-22. In figures V-20, V-21, and V-22 the QPM and QCD calculations corresponding to the data are also plotted. The modeis generally agree with each other in their predictions for the phenomena examined here, and with the data as well. The small differences between the QPM and QCD planarity predictions primarily come from the different multiplicity distributions of the models.

QCD predicts that the hadron transverse momentum due to hard givon bremsstrahiung should be found in the piane formed by the quark, gluon, and diquark momenta. If the high transverse momentum observed in high $\pi_{F}$ events is due to hard gluon bremsstrahlung, then the high $\pi_{F}$ events should have more planar hadron systems than other events, and, therefore, higher planarity. In the $\left(\pi_{F}, P\right)$ scatter plot for the $W^{2}>50 \mathrm{GeV}^{2}$ events, figure $\mathrm{V} \cdot 23$, high $\pi_{F}$ events are clearly more planar than those in the bulk of the sample. For $\pi_{F}<2.0,\langle P\rangle=0.51 \pm 0.01$; for $\pi_{F}>3.0$, $\langle P\rangle=0.73 \pm 0.02$.

Although high $\pi_{F}$ events should have high planarity if they are hard giuon bremsstrahlung events, there may be some trivial explanation for the observed correlation, or some non-QCD dynamic explanation. Having established that planarity is strongly multiplicity dependent, we compare the charge multiplicity distributions of the $\pi_{F}<20$ events and of the $\pi_{F}>3.0$ events in figure V-24, The higher $\pi_{F}$ events have a slightly higher average charge multiplicity, $\left\langle N_{C H}\right\rangle=7.4 \pm 0.3$, than do the lower $\pi_{F}$ events, $\left\langle N_{C H}\right\rangle=7.1 \pm 0.1$, so the higher planarity of the higher $\pi_{F}$ events is not a multiplicity effect. The planarity distribution for the $\pi_{F}>3.0$ events is plotted in figure V-25 along with the corresponding histograms from the QPM and QCD Monte Carlos. The Monte Carlo histograms both have more events at very high planarity than does the data histogram. This is probably due to the lower multiplicities of the Monte Carlo events. Unlike the data, Monte Carlo high $\pi_{F}$ events have lower than average multiplicity. $\left\langle N_{C H}\right\rangle=5.8 \pm 0.1$ in the QCD Monte Cario and $\left\langle N_{C H}\right\rangle=6.0 \pm 0.3$ in the QPM Monte Carlo.

The high planarity of high $\pi_{F}$ events also does not come simply from the high transverse 
momentum in these events. By increasing or decreasing all transverse momenta in an event by the same multiplicative factor, one can generate (almost) any value of $\pi_{F}$ without changing the event's planarity. However, any mechanism which produces a hadron on the tail of the transverse momentum distribution, that is, with much greater than typical transverse momentum, is likely to produce high $\pi_{F}$, high planarity events preferentially. Denote by $\vec{P}_{1}$ the momentum of such a high transverse momentum hadron, and by $\bar{P}_{T !}$ its transverse momentum. Other hadrons in the events must have transverse momenta, $\vec{P}_{\pi}$, to balance $\vec{P}_{T 1}$ :

$$
\sum_{i>1} \vec{P}_{\pi i} \cdot \frac{\vec{P}_{T 1}}{\left|\vec{P}_{T 1}\right|}=-\left|\vec{P}_{T 1}\right|
$$

To the extent that $\left|\vec{P}_{T 1}\right|$ is much greater than typical transverse momenta, the other transverse momenta in the $\left(\dot{q}, \vec{P}_{1}\right)$ plane, $\vec{P}_{T \cdot} \cdot \frac{\vec{P}_{T 1}}{\left|\vec{P}_{T 1}\right|}$, will tend to be greater than typical transverse momenta. At the same time, the components of the $\vec{P}_{\pi}$ out of the $\left(\vec{q}_{,} \vec{P}_{1}\right)$ plane should be typical. If the mechanism which produces a hadron on the tail of the transverse momentum distribution is hard enough to yield high $\pi_{F}$, it is also likely to yield high planarity. Hard gluon bremsstrahlung is obviously one such process, but it is not the only possibility.

High $\pi_{B}$ events, which have high transverse momentum in the backward direction, also are more planar than most events: see the $\left(\pi_{B}, P\right)$ scatter plot for the $W^{2}>50 \mathrm{GeV}$ events, figure V-26. This correlation is not explained by the hard gluon bremsstrahlung hypothesis. Similarly, the high $\pi$ events in the $\left(\pi_{F}, P\right)$ and $\left(\pi_{B}, P\right)$ scatter plots for the $W^{2}<50 \mathrm{GeV}^{2}$ events, figure $\mathrm{V}-27$, are more planar than most events, and in this kinematic region hard gluon bremsstrahiung is calculated to be unimportant. These results are summarized in figure V-28 where average planarity is plotted as a function of $\pi$ for each of the data samples considered in figures V-23, V-26, and V-27.

The QPM calculations of $\langle P\rangle$, also shown in figure V-28, describe the data fairly well, and provide an explanation for the planarity of high $\pi$ events. In this model high $P_{T}$ hadrons come from high $k_{T}$ quark-antiquark pairs bubbling up from the vacuum (see Chapter IV, section 3). The distribution of the maximum $k_{T}$ value in an event is shown in figure $\mathrm{V}-29$, with the $\pi_{F}>3.0$ contribution shaded in. Because the maximum $k_{T}$ values in high $\pi$ events (typically $800 \mathrm{MeV}$ ) are so much 
greater than typical hadron transverse momentum values, most of the hadron transverse momentum in these events will be produced in the plane defined by $\dot{q}$ and the quark-antiquark relative momentum.

This QPM mechanism for giving high $\pi_{F}$ and high $\pi_{B}$ events high planarity is similar; in many respects, to the $Q C D$ mechanism for giving high $\pi_{F}$ high planarity, although different in details. In the QCD case a quark and gluon have large relative transverse momentum; in the QPM case a quark and antiquark have large relative transverse momentum. In the QCD case the quark and giuon are both forward; in the QPM case the quark and antiquark may be either backward or forward. Yet in both models pairs of high transverse momentum partons produce correlated high transverse momentum hadrons, which in turn yield both high $\pi$ and high planarity. For this reason the observed correlation of high planarity with high $\pi$ does not, by itself, favor QCD over all other models. It does, however, exclude models which produce high $\pi$ events as a result of an overall increase in the transverse momentum scale.

This point can be illustrated by calculating the $\left(\pi_{F}, P\right)$ distribution for a modified two-jet model in which only the magnitudes of the transverse momenta have been changed. For the $\left(\pi_{F}, P\right)$ scatter plot (and its projections) shown in figure V-30, $\sigma_{q}$, the Field and Feynman parameter giving the width of the $k_{T}$ distribution, has been changed from $0.380 \mathrm{GeV}$ to $0.760 \mathrm{GeV}$. The resulting $\pi_{F}$ distribution has the bulk of its entries above zero, and there is a larger fraction of events with $\pi_{F}>$ 3.0 than observed in the data or in the standard QPM and QCD models. Events with $\pi_{F}$ somewhat greater than 3.0 show a normal planarity distribution, unlike the data or either standard model. Only events with $\pi_{F}$ on the far tail of the distribution, in this case with $\pi_{F}$ greater than about 4.5 , are more planar than usual. Generally, increasing the magnitudes of all transverse momenta in events produces high $\pi$, normal planarity events, while increasing some transverse momenta through an underlying hard process produces high $\pi$, high planarity events. In the data the high $\pi_{F}$ events are also high planarity events. Thus one may conclude that the net signal of high $\pi_{F}$ events (over that expected from forward-backward symmetry) results from a hard process. 


\section{Angular Energy Flow}

QCD is a field theory of strong interactions whose underlying quanta are quarks and gluons. Perturbation theory allows one to make predictions for short distance phenomena, such as hard gluon bremsstrahlung, but one needs other tools (not yet discovered) to calculate longer distance phenomena such as structure and fragmentation functions. Phenomenological models allow one to describe the longer distance behavior in QCD reasonably well, but the less an experimental result depends on a particular model for its interpretation, the stronger is that result. Basham, Brown, Ellis, and Love ${ }^{18}$ introduced angular energy flow as a measure of jetty final states which is relatively insensitive to the evolution of partons into hadrons.

Angular energy flow in the hadron CM system is $\frac{1}{\Sigma} \frac{d \Sigma}{d \vec{\Omega}}$, where $\frac{d \Sigma}{d \vec{\Omega}}$ is an energy weighted cross section per unit solid angle. The weighting factor is taken so that

$$
d \Sigma \mp\left(2 E^{C K} / W\right) \cdot d \sigma
$$

where $E^{C M}$ is the sum of the energies of all particles moving in the relevant direction. With this normalization (chosen so that the quark and diquark in a two-jet system would each have energy fraction, $2 E^{C H} / W$, equal one)

$$
\int d \vec{\Omega} \frac{d \Sigma}{d \vec{\Omega}}=2 \sigma_{t o t}
$$

Angular energy flow therefore measures the fraction of total energy emitted per unit solid angle. To the extent that all hadrons in a parton's jet have momenta parallel to that of the parton, and the energies of the hadrons in the jet sum to the energy of the parton, the angular energy flow of the hadron final state will be the same as that of the parton final state.

The angular energy flow for the $W^{2}>50 \mathrm{GeV}^{2}$ sample as a whole is projected onto the $\left(\hat{q}_{v i s}, \hat{u}_{1}\right)$ plane in figure V-31a and onto the $\left(\hat{q}_{v i s}, \hat{u}_{2}\right)$ plane in figure V-31b. (The orientation of $\hat{u}_{1}$ in each event is chosen so that $\hat{u}_{1} \cdot \vec{P}_{\mu}^{T}>0$, and the orientation of $\hat{u}_{2}$ so that $\dot{u}_{2}=\hat{q}_{\text {is }} \times \hat{u}_{1}$.) Both plots show an apparent two-jet structure, as if each of two partons, moving in opposite directions, had evolved into a jet of finite width. Because the plots are sums over many events, such an interpretation is too simple. However, the shapes seen are generally consistent with those one 
expects in a two-jet model. The QPM curves, also plotted in figure V-31, show a similar two-jet structure, although in detail they do not describe the data. Near $\pm \frac{\pi}{2}$, the calculated (QPM) angular energy flow is greater than that observed.

This discrepancy between the model and the data results from the model's failure to describe hadron production at small $\left|x_{F}\right|$ correctly. The Field and Feynman single quark jet model is constructed to describe high energy jets in which longitudinal momenta are greater than transverse momenta and hadron masses. For the QPM calculations, TUBES generates two Field and Feynman jets in the hadron CM system of each event. These jets are tied of near $x_{F}=0$, where longitudinal momenta take on magnitudes comparable to those of transverse momenta and hadron masses. The details of this procedure define the properties of the model's centrally produced hadrons. Because the QPM calculations do not reasonably describe the angular energy flow at large angles, while they do describe the angular energy flow at smaller angles in the forward direction, one must conclude that something is wrong with the model's description of central hadron production.

The difficulty is apparent in figure V-32, where the $x_{F}$ distribution for charged particles and the corresponding QPM and QCD Monte Carlo curves are shown. TUBES generates too many central hadrons. This failure of the Monte Cario does not affect most of our analysis. Generally, our conclusions rest on features of the $\left|x_{F}\right|>0.05$ tracks, and central meson production does not affect these substantially. To the extent that our conclusions depend on the transverse momentum of $\left|x_{F}\right|<0.05$ tracks, and not their number, the Monte Carlo does well. In figure V-33 $<p \frac{2}{T}>$ of charged tracks is plotted as a function of $x_{F}$ for the $W^{2}>50 \mathrm{GeV}^{2}$ data. The QPM and QCD curves are both superposed. For $\left|x_{F}\right|<0.05$ the two curves are very close and are both consistent with the data.

The QCD curves also describe the angular energy flow data plotted in figure V-31. The QCD and QPM curves are similar, althcugh the QCD curves are a bit fatter in the forward direction of the $\left(\dot{q}_{v i s}, \dot{u}_{1}\right)$ projection. This reflects the higher transverse momentum of hadrons from three-jet events. To the extent that the QCD Monte Carlo describes this data better than does the QPM Monte Carlo, a two-jet model with higher transverse momentum hadrons could be constructed to fit 
the data just as well.

The QPM and QCD calculations for angular energy flow are similar because the typical width of a single jet is greater than the typical angular separation of the quark and gluon momenta in $W^{ \pm} q \rightarrow q g$ events. Only in very hard gluon bremsstrahlung events does one expect the quark and gluon jets to be well separated. These events should also have high $\pi_{F}$ and high planarity, so we proceed to plot the angular energy flow of the $\pi_{F}>3.0, P>0.5$ events in figure V-34. The $\left(\hat{q}_{\text {vis }}, \hat{u}_{1}\right)$ projection shows a distinct three-jet shape, two jets forward and one backward, while the $\left(\dot{q}_{\text {vis }}, \dot{u}_{2}\right)$ projection shows a two-jet shape, one jet forward, one backward. This is exactly what one expects for three co-planar jets. As most $\pi_{F}>3.0, P>0.5$ events in the QCD Monte Carlo are generated as three-jet events, it is no surprise that the QCD curves, also plotted in figure V-34, mimic the shapes observed in the data.

Perhaps surprisingly, the QPM curyes look much the same. This derives from the limited phase-space occupied by high $\pi_{F}$, high planarity events. Viewed in the hadron CM system, the forward tracks in the events have high transverse momentum and limited longitudinal momentum, so are found at fairly large angles. This is best illustrated by doing some simple calculations.

The minimum $\left\langle\left|P_{T}\right|>\right.$ required to yield $\pi_{F}>3.0$ is calculated as a function of contributing multipicity, $n_{F}$, in Table V-3. By construction, they are all much greater than $P_{T 0}, 0.320 \mathrm{GeV}$. Longitudinal momentum in the hadron CM system is given by

$$
P_{L}^{C M}=x_{F} W / 2
$$

With $W^{2}=144 \mathrm{GeV}^{2}$ and $x_{F}=0.2$, typical values for these variables, $P_{L}^{C M}=1.2 \mathrm{GeV}$. A track with transverse momentum $0.753 \mathrm{GeV}$ (this value taken from Table $\mathrm{V}-3$ for $n_{F}=3$ ) and longitudinal momentum $1.2 \mathrm{GeV}$ makes an angle with $q$

$$
\theta=\tan ^{-1}(0.753 / 1.2)=32^{\circ} .
$$

This is greater than the angular spread observed in the bulk of the data, see figure V-31, and typical of the angular separation of the forward jet-like structures observed in the high $\pi_{F}$, high planarity sample, see figure V-34.

The $\pi_{F}>3.0$ events are highly planar, $\langle P\rangle=0.73 \pm 0.02$. Most of the transverse 
momentum is therefore found in the $\left(\hat{q}_{v i s}, \dot{u}_{1}\right)$ plane rather than in the $\left(\dot{q}_{v i s}, \dot{u}_{2}\right)$ plane; with $P=$ $0.73,\left\langle P_{T 1}^{2}\right\rangle \approx 6\left\langle P_{T 2}^{2}\right\rangle$ (see equation V.5). As a result, the large transverse momentum discussed in the preceding paragraph generates a three-jet structure in the $\left(\hat{q}_{v i s}, \hat{u}_{1}\right)$ plane and a two-jet structure in the $\left(\dot{q}_{v i s}, \dot{u}_{2}\right)$ plane.

The conciusion to be drawn from the above analysis, that high $\pi_{F}$, high planarity events characteristically have three-jet angular energy flow, independent of underiying physics, is easily generalized. Every region of $\left(\pi_{F}, P\right)$ has a characteristic angular energy flow shape, independent of underiying physics. This is true as long as the overall energy scale, $W^{2}$, is fixed and as long as the longitudinal momentum distribution is fixed.

This phase-space analysis allows one to count, to first order, the number of events with some characteristic shape by counting the number of events in the appropriate region of $\left(\pi_{F}, P\right)$. In particular, counting the number of high $\pi_{F}$, high planarity events corresponds to counting the number of three-jet events. Because events with three-jet angular energy flow may not have an underlying three parton structure, one must determine the backgrounds to a real three-jet signal either theoretically or experimentally. In either case some assumptions are necessary.

One can determine the backgrounds to the real three-jet signal experimentally by assuming that conventional physics is essentially forward-backward symmetric and correcting for instrumental effects. In section 5 of this chapter the number of high $\pi_{F}$ and high $\pi_{B}$ events were compared (see Table V-2). For $W^{2}<50 \mathrm{GeV}^{2}$ the data are consistent with the forward-backward symmetry hypothesis. For $W^{2}>50 \mathrm{GeV}^{2}$ there is a net signal of $49 \pm 9$ events with $\pi_{F}>3.0$ and $P>0.6$. This can now be identified as a net signal of three-jet events. 
Chapter VI: Interpreting the Data in the QCD Model

At low $W^{2}$, transverse momentum production is forward-backward symmetric. At high $W^{2}$, it is not. Hard gluon bremsstrahlung, as predicted by $Q C D$, provides a natural explanation for the observed phenomena. Assuming it is the correct explanation, we can turn back to the Monte Carlo calculations and ask what they have to say about the underlying process.

The basic questions about three-jet events are straightforward. How many are generated, and what are their characteristics? Almost equally straightforward, how many do we detect, and what are their characteristics? Most three-jet events generated by the QCD Monte Carlo do not distinguish themselves from the two-jet sample; only those with high quark-gluon invariant mass and high parton transverse momentum feed the high $P_{T}$ signals. To first order, the rate of three-jet production and the characteristics of the three-jet events produced are separate issues. The three-jet crosssection is proportional to $\alpha_{s}$, and $\alpha_{s}$ varies only slowly with $x_{p}$ and $z_{q}$, the QCD variables which determine the three-jet characteristics of the $W^{ \pm} q \rightarrow q g$ events (see equation II.32).

\section{The Three-jet Rate and $\alpha_{s}$}

One determines the rate of hard gluon bremsstrahlung events from its effect on $P_{T}$ distributions, and from this determines $\alpha_{s}$. We varied $\Delta^{2}$ in equation II.32 until the Monte Carlo calculations agreed reasonably well with the $\Delta P_{T}^{2}$ data and the high $\pi_{F}$ data. (See Chapter II, section 8 and Chapter VI, section 1 for more quantitative discussions of $\Lambda^{2}$ and $\alpha_{s}$.) The Sudakov form factor differs significantly from one for most of our quark-gluon invariant mass spectrum, so in determining an appropriate value of $\alpha_{s}$ we must ask our questions carefully.

Most three-jet events look very much like two-jet events. With $\Lambda^{2}$ adjusted as described above, $20 \%$ of the events with $W^{2}>50 \mathrm{GeV}^{2}$ were generated as three-jet events. Yet only a quarter of these are detected with $\pi_{F}>3.0$, where the three-jet signal is experimentally defined. These high $\pi_{F}$ events also contribute most of the $\Delta P_{T}^{2}$ signal, so in practice it is these events that determine the value of $\alpha_{s}$ necessary to have the Monte Carlo describe the data. We therefore consider only the $\pi_{F}$ $>3.0, W W_{q} \rightarrow q g$ events in determining the appropriate value of $\alpha_{s}$. 
With $\Lambda^{2}$ adjusted in the QCD Monte Carlo, $W=q \rightarrow q g$ events with $W^{2}>50 \mathrm{GeV}^{2}$ and $\pi_{F}>$ 3.0 have $\left\langle\alpha_{s}\right\rangle=0.29$. The greatest single uncertainty we assign to this value comes from the statistical error in the net three-jet rate observed experimentally. This signal should be directly proportional to $\left\langle\alpha_{s}\right\rangle$, so the $15 \%$ uncertainty in the observed three-jet rate (see Chapter V, section 5 and Table V-2) translates into a 0.05 absolute error in $\left\langle\alpha_{s}\right\rangle$. The remaining uncertainties are systematic, and more difficuit to estimate accurately.

Four sources of systematic error in determining the three-jet rate are easily identified. The parameterization of jet evolution in the Monte Carlo is uncertain. The Monte Carlo simulation of the detector's response and resolution is not exact. The subtraction of the two-jet background under the three-jet signal assumed that the two-jet sample is exactly forward-backward symmetric. The effects of re-interaction of the hadron final state with the neon nucleus must be considered.

\subsection{The Uncertainty in Jet Parameterization}

The parameterization of jet evolution will affect the correspondence between the underlying three-jet rate and the observed three-jet rate because $\pi_{F}$ and planarity depend on the evolution of quark and gluon jets. In analyses of $e^{+} e^{-}$data using similar Monte Carlos, groups at PETRA ${ }^{19}$ bave carefully studied the corresponding question and conciuded that the uncertainty in the three-jet rate from this source is about 10\%. Having varied some of the jet evolution parameters in our Monte Carlo, but in a less systematic way, we believe this $10 \%$ number is also appropriate for our analysis. This gives us an uncertainty of about 0.03 in $\left\langle\alpha_{s}\right\rangle$.

\subsection{The Uncertainty in Modelling the Detector}

The Monte Carlo simulation of our detector is not exact, and may therefore contribute some uncertainty into the correspondence between the events' true characteristics and what we observe. For charged tracks we have checked that the track length and momentum distributions of the Monte Carlo agree with those of the data. For neutrals we have similarly checked the conversion distance distributions. The average energy correction factor, which depends on the relative production and detection of charged and neutral hadrons, is the same in the Monte Carlo as in the data Further, 
varying the resolution-related cuts in the analysis and the resolution related parameters in the Monte Carlo have little effect on our results. Because our analysis is insensitive to the fine details of the detector's response, we estimate the contribution to the systematic uncertainty in $\left\langle\alpha_{s}\right\rangle$ due to uncertainty in detector response to be $5 \%$ or less, about 0.015 absolutely.

\subsection{The Uncertainty in Subtracting the Two-Jet Background}

At low $W^{2}$ our pristine Monte Cario produces a two-jet event sample which is forwardbackward symmetric in transverse momentum production, and our smeared Monte Carlo correctly describes the data. While we have assumed that two-jet production is exactly forward-backward symmetric, we may extrapoiate the observed uncertainty in this hypothesis from low $W^{2}$ to high $W^{2}$. At low $W^{2}$ the difference in the rate of high $\pi_{B}$ events and high $\pi_{F}$ events (after correcting for instrumental effects) is $(0.3 \pm 0.3) \%$. As the net rate of high $\pi_{F}$ events at high $W^{2}$ is roughly $3 \%$, this translates to a systematic uncertainty in $\left\langle\alpha_{s}\right\rangle$ of 0.03 .

Forward-backward asymmetric processes, other than hard gluon bremsstrahlung, which produce high $\pi_{F}$, high planarity events at high $W^{2}$, but not low $W^{2}$, may also exist. Charm production and subsequent decay is often considered a candidate for such a process. Asymptotically, charmed particles are produced in roughly $10 \%$ of neutrino-nucleon interactions, and are produced predominantly in the forward direction of the hadron system. However, the transverse momentum of hadrons produced in charm decays is not sufficient to produce the $\Delta P_{T}^{2}$ and high $\pi_{F}$ signals we observe. In this experiment we have observed a dilepton signal ${ }^{3,11}$ in which the second lepton presubably comes from the semi-leptonic decay of charm. The transverse momenta of the second lepton and of $K^{0 \prime} \mathrm{s}$ in the dilepton events are not substantially greater than the transverse momenta of hadrons in all events.

The invariant masses of charmed particles are about $2 \mathrm{GeV}$. The decay of a $2 \mathrm{GeV}$ particle does not produce enough transverse momentum to generate $\pi_{F}>3.0$. As will be discussed in section 2 of this chapter, for three-jet events, it is only when the quark-gluon invariant mass exceeds 3.5 GeV that the three-jet signal really begins to distinguish itself from the two-jet background. Only when a heavy particle has a comparable mass will its decay generate a comparable signal. The QPM and QCD Monte Carios both include charm production from down quarks (Cabibbo- 
suppressed, with $\sin ^{2} \theta_{C}=0.05$ ) and from strange quarks (Cabibbo-favored). Even in the pristine two-jet Monte Cario, this charm production has no observable effect on $\Delta P_{T}^{2}$ or on the high $\pi_{F}$ rate. We have no evidence in this experiment for the production of higher mass particles, such as those containing bottom quarks, so we judge the contribution to the observed forward-backward asymmetry from heavy particie production to be negligible.

\subsection{Uncertainties Which Result from Using a Neon Target}

The target in this experiment was primarily neon, not hydrogen. This has been accounted for only partially in the analysis of the data and in the Monte Carlo calculations. The first effect of the neon is that the target nucleons are not at rest in the lab; they have Fermi momentum of order several hundred $\mathrm{MeV}$. This has been included in the Monte Cario and has little effect on most of the results. The resolution in kinematic variables such as $Q^{2}$ and $W^{2}$ is a bit worse, but variables such as $\pi$ and planarity remain largely unaffected as they are measured with respect to the visible hadron direction, which naturally includes the Fermi momentum of the target nucleon.

The more worrisome effect of neon is that one or more of the final state hadrons may interact with the remaining nuclear material, thus changing the observable hadron final state. The fraction of events with such a re-interaction is large in this experiment ${ }^{20}$, so this effect must be considered. As with other hadronic interactions, these re-interactions should be of low momentum transfer mostly. Because energy and momentum are conserved, the only effects on resolution in kinematic variables are due to our detecting some different fraction of the hadron final state momentum. When there is some momentum transfer between the original hadron final state and the remaining nuclear material, the $x_{F}>-0.7$ requirement used in defining $\vec{Q}_{v i s}$ may exclude the $\vec{P}_{T}$ transferred to the nuclear material, smearing the hadron system resolution in $P_{T}$. The hadron system resolution in $P_{L}$ is not affected so much because $x_{F}<-0.7$ hadrons are very slow in the lab. Although the physics is somewhat different, the effect on resolution is similar to the smearing from Fermi motion. Thus, we increased the magnitude of the Fermi motion in the Monte Carlo until the resolution in the angle between the visible hadron direction and the lepton plane, $\theta_{\text {our }}$, agreed with that observed in the data (see figure III-8). 
Even with the resolution unchanged, re-interactions may increase or decrease the transverse momentum of observable hadrons. Most re-interactions must be quasi-elastic since very few negative hadrons are produced near $x_{F}=-1$. In such interactions we expect the momentum transfer between the final state hadron and the nuclear material to be small and uncorrelated with the transverse momentum of the hadron. The net effect will be a small additional smearing, but no substantial bias. Inelastic scattering splits the transverse momentum of the original hadron among its interac: tion products, which tends to diminish average transverse momentum. It also introduces an additional smearing from the momentum created perpendicular to the interaction direction, which is typically several hundred $\mathrm{MeV}$ per particle and which sums to zero in each event. We therefore expect hadronic re-interactions to produce no substantial biases in our transverse momentum studies. Experimentally, our $\left\langle P_{Q U T}^{2}\right\rangle_{F}$ and $\left\langle P_{O U T}^{2}\right\rangle_{B}$ results agree well with those from a similar neutrino-deuterium experiment ${ }^{21}$ in which the hadronic re-interaction rate would be much smaller. We estimate the systematic error in $\left\langle\alpha_{s}\right\rangle$ due to hadronic re-interactions to be less than $10 \%$, about 0.03 .

\section{Limitations of the QCD Model}

The value of $\alpha_{s}$ quoted is appropriate to the QCD model embodied in the Monte Cario calculations. Specifically, it is the strong coupling constant for the cross-section of Eq. II-27, which is a modified first order perturbative result. A second order calculation, or one which damps the colinear divergences using a technique other than the Sudakov form factor, or one where the argument of $\alpha_{s}$ is other than $M_{q g}^{2}$ may yield a different relation between $\alpha_{s}$ and the $W=q \rightarrow q g$ rate. Such modified calculations might also yield different $\left(x_{p}, z_{q}\right)$ spectra, even for the same integrated rate. This would affect the ratio between $\alpha_{s}$ and the number of observed three-jet events because the fraction of events with $\pi_{F}>3.0$ increases with $M_{q g}$ and $P_{T P}$, which both depend on $x_{p}$ and $z_{q}$. Rather than assign a systematic error due to the QCD model to the value of $\alpha_{s}$, it is appropriate to display explicitly the correspondence between the data and the underiying variables measured by $\alpha_{s}$ in the model. In figure VI-1 the $M_{q g}$ spectrum is plotted for all $W=q \rightarrow q g$ events with $W^{2}>50 \mathrm{GeV}^{2}$ and passing cuts. Shaded in is the spectrum for those with $\pi_{F}>3.0$ and $P>0.6$. The efficiency 
with which $\pi_{F}>3.0, P>0.6$ identifies $W{ }_{q} \rightarrow q g$ events is the ratio of the shaded spectrum to the full spectrum, which is plotted in figure VI-2. The corresponding plots for $P_{T P}$ are shown in figures VI-3 and VI-4. $\alpha_{s}$ is proportional to the integrated rate of $W^{ \pm} q \rightarrow q g$ events, with the efficiency as a function of $M_{q g}$ or $P_{T P}$, as shown. For any variation of the QCD model, one may calculate the variation's $M_{q g}$ or $P_{T P}$ spectrum, the experimental efficiency for observing that spectrum using our $\left(\pi_{F}, P\right)$ cut, and the value of $\alpha_{s}$ in that model corresponding to our three-jet rate (Table V-2).

\section{Event Characteristics}

How are three-jet events like two-jet events, and how are they different? To what extent do event characteristics vary with the underlying variables $M_{q g}$ and $P_{T P}$ ? These are questions easily asked of the Monte Carlo.

The first set of characteristics one can study is that of event variables which do not depend on the detailed structure of the hadron final state. As a first example, the $W^{2}$ spectra for two and three-jet events with $W^{2}>50 \mathrm{GeV}^{2}$ are plotted in figure VI-5; the $W^{2}$ spectra for three ranges of $M_{q g}$ are plotted in figure VI-6a, and for three ranges of $P_{T P}$ in figure VI-6b. Similar plots of the $E_{m} \nu, Q^{2}, y, x_{B j}, \xi$, and $s$ spectra are show in figures VI-7 through VI-20. As three-jet events have large invariant mass quark-gluon pdirs, three-jet events tend to be harder in most kinematic variables than do two-jet events.

Averages from these distributions are collected in Table VI-1. Hard gluon bremsstrablung is strongly correlated with higher energy scales, but not with the scaling variables $x_{B j}$ and $y$. The strongest correlation is with the neutrino-quark $C M$ energy squared, $s=2 m_{N} E$, $\xi$, which cannot be measured experimentally. The correlation with the measurable quantities $E_{\eta}, \eta_{v} Q^{2}$, and $W^{2}$ is weaker, and the three-jet events with the lowest values of $M_{q g}$ and $P_{T P}$ actually have somewhat lower energies, etc., than does the aggregate of the two-jet events. The scaling variable $x_{B j}$ does not correlate with hard gluon bremsstrahlung because it is the product of two terms, $\xi$ and $\left(1-x_{p}\right)$, of which the first correlates with hard giuon bremsstrablung and the second anti-correlates. The scaling variable $y$ does not correlate with hard gluon bremsstrahlung because it measures the scattering 
angle of the lepton in the neutrino-quark $\mathrm{CM}$, whose distribution depends on whether the projectile is a neutrino or an antineutrino and whether the target is a quark or an antiquark, but not on whether the final state quark is real (as in a two-jet event) or virtual (as in a three-jet event).

The second set of characteristics one can study is that of event and track variables which do depend on the detailed structure of the hadron final state. Because instrumental effects bias the determination of these variables, we shall consider, in addition to the pristine distributions, the corresponding smeared distributions. The pristine distributions tell the truth about the model; the smeared distributions tell how we would see the two- and three-jet samples of the model.

In Chapter $\mathrm{V}$ the model predictions for $\left\langle P_{T}^{2}\right\rangle_{F(B)}$ as functions of $W$ were compared with the data. In figure VI-21 we plot pristine $\left\langle P_{T}^{2}\right\rangle_{F}$ vs $W$ for two-jet events and for several ranges of $M_{q g}$ for three-jet events. In figure VI-22 we plot the corresponding curves from the smeared Monte Carlo. In the pristine plot, $\left\langle P_{T 2}\right\rangle_{F}$ decreases slightly with $W$ for any range of $M_{q g}$ (except the two-jet events, $M_{q g}=0$ ). For fixed $M_{q g}$ phase-space requires the quark and gluon to be at wider angles with respect to $\hat{q}$ at low $W$ than at high $W$. At low $W$ a higher fraction of the hadrons from the quark and giuon jets fail the $x_{F}>0.05$ cut, which explains the effect noted. In the smeared plot, $\left\langle P_{T 2}\right\rangle_{F}$ increases with $W$ for any range of $M_{q g}$. This (anti-) correlation is an instrumental effect. As noted earlier, we tend to measure $\dot{q}$ better at high $W$ than at low $W$ (see figure III-7), and as a result of our cuts, we tend to measure $\left\langle P_{T 2}\right\rangle_{F}$ too small (see Chapter $V$, section 4 ). The worse we measure $\dot{q}$, the more biased is our measurement of $\left\langle P_{T 2}\right\rangle_{F}$, which is what we are seeing here.

Several substantial conclusions may be drawn from figures VI-21 and VI-22. First, $W=q \rightarrow q g$ events do produce high forward transverse momentum, and this effect increases with $M_{q g}$. Second, because most events are generated in the model as two-jet events, $\left\langle P_{T}^{2}\right\rangle_{F}$ is dominated by tracks coming from two-jet events, and the modulation due to three-jet events is not as strong as might be inferred otherwise. Third, while smearing does not qualitatively affect the differences in $\left\langle P_{T}^{2}\right\rangle_{F}$ between the types of events studied here, it does diminish the differences.

In figure VI-23 we plot the pristine $\pi_{F}$ distributions for two-jet events and for three ranges of 
$M_{q g}$ for three-jet events. The smeared $\pi_{F}$ distributions are shown in figure VI-24. Similariy, the corresponding planarity distributions are plotted in figures VI-25 and VI-26. Both $\pi_{F}$ and planarity tend to increase with increasing $M_{q g}$, but their distributions at any value of $M_{q g}$ are so wide that they overiap substantially with the distributions from other values of $M_{q g}$. Only at the highest values of $\pi_{F}$ may one infer that the underlying structure of an event's hadron system is likely to be three-jet. Although the differences between the $\pi_{F}$ and planarity distributions for various $M_{q g}$ samples are diminished by smearing, the qualitative features of the pristine distributions are also found in the smeared distributions.

Only events with high quark-gluon invariant mass and high parton transverse momentum contribute substantially to the high $P_{T}$ signals we observe. As $\alpha_{s}$ was adjusted so that the Monte Carlo would correctly describe the high $\pi_{F}$, high planarity signal, the $W^{ \pm} q-q g$ events contributing to this signal are of special interest. The average value of $\alpha_{s}$ in these events is 0.29 ; similarly $\left\langle M_{q g}^{2}\right\rangle \approx 25 \mathrm{GeV}^{2},\left\langle P T_{T P}^{2}\right\rangle \approx 10 \mathrm{GeV}^{2}, \quad\langle\hat{s}\rangle \approx 100 \mathrm{GeV}^{2}, \quad\left\langle Q^{2}\right\rangle \approx 45 \mathrm{GeV}^{2}$, and $\left\langle W^{2}\right\rangle \approx 155 \mathrm{GeV}^{2}$. The Monte Carlo calculations all use $M_{q g}^{2}$ as the momentum scale with which $\alpha_{s}$ varies and to which $\Lambda_{e f f} \approx 500 \mathrm{MeV}$ corresponds. If one believes that $\alpha_{j}$ varies with some other momentum scale, then to first order the value of $\Lambda_{e f f}$ may be calculated from $\alpha_{s}=0.29$ and the average value of the variable being considered. Because our spectrum in any such variable spans a large range, in the sense than $\ln \left(\mu^{2} / \Delta^{2}\right)$ varies substantially over the range, a more correct determination of $\Lambda_{f f}$ requires that the Monte Carlo calculations be performed with $\alpha_{s}$ varying with the momentum scale of interest.

The net rate of three-jet events we observe experimentally corresponds to

$$
\alpha_{s}=0.29 \pm 0.05 \pm 0.05
$$

for the QCD model embodied in the Monte Carlo. The first error quoted is the statistical uncertainty derived from the net number of three-jet events observed. The second error is the sum in quadrature of the systematic errors discussed above. No error is assigned to the model itself, so great care must be exercised in comparing this value of $\alpha_{s}$ with those derived in other models.

Three-jet events observed in $e^{+} e^{-}$interactions at PETRA ${ }^{19.22}$ have also been interpreted as 
hard gluon bremsstrahlung events, $e^{+} e^{-}-q \overline{q g}$. Values of $\alpha_{s}$ deduced from the three-jet rates using procedures similar to ours range from 0.16 to 0.23 . While these values of $\alpha_{s}$ are lower than our value of $\left\langle\alpha_{s}\right\rangle$, the quark-gluon invariant masses in the PETRA events are typically greater than the quark-gluon invariant masses in our events. At the PETRA energies (near $s^{1 / 2}=30 \mathrm{GeV}$ ) $<M_{q g}^{2}>\approx 150 \mathrm{GeV}^{2}$, so taking $\alpha_{s}=0.20$ for PETRA, equation II.32 gives $\alpha_{s}=0.27$ for $M_{q g}^{2}=$ $25 \mathrm{GeV}^{2}$. Thus, our value for $\left\langle\alpha_{s}\right\rangle$ is in accord with the PETRA values. Somewhat more generally, the net rate of three-jet events in high $W^{2}$ neutrino-nucieon interactions is roughly the same as the net rate of three-jet events in high energy $e^{+} e^{-}$interactions. 


\section{Capter VII: Summary and Conclusions}

We have studied high energy neutrino-nucleon interactions for evidence of the hard gluon bremsstrahlung process predicted by QCD. We have compared our data with QPM and QCD Monte Carlo calculations which included simulation of the detector's response and resolution. We observe high transverse momentum signals consistent with the QCD model, but not with the QPM model; instrumental biases were such as to diminish any real signal rather than to create a signal.

We confirm earlier observations that the transverse momentum of hadrons increases with $W^{2}$. This is true for both forward and backward hadrons (in the hadron system CM), and does not depend, qualitatively, on the magnitude of $x_{F}$ used to define forward and backward. Above $W^{2} \approx 100 \mathrm{GeV}^{2},\left\langle P_{T}^{2}\right\rangle_{F}$ grows more than does $\left\langle P_{T}^{2}\right\rangle_{B}$, so that $\Delta P_{T}^{2}=0.047 \pm 0.011 \mathrm{GeV}^{2}$ where the QPM Monte Carlo calculations, which correctly describe the data at low $W^{2}$, predict -0.03 (which is non-zero due to instrumental effects). While the symmetric growth of $\left\langle P_{T}^{2}\right\rangle_{F}$ and $\left\langle P_{T}^{2}\right\rangle_{B}$ below $W^{2} \approx 100 \mathrm{GeV}^{2}$ is consistent with the growth of phase-space (as demonstrated by the QPM calculations), the asymmetry at high $W^{2}$ implies a contribution to $\left\langle P_{T}^{2}\right\rangle_{F}$ from some other source.

In an effort to identify a sample of high $P_{T}$ events, we defined the variable $\pi_{F}$, which measures the forward transverse momentum in an event, and the corresponding variable $\pi_{B}$, which measures the backward transverse momentum in an event. As with $\left\langle P_{T}^{2}\right\rangle$, which measures averages over tracks, we studied $\langle\pi\rangle$, which measures averages over events. At low $\left.W^{2},<\pi\right\rangle_{F}$ and $\left.<\pi\right\rangle_{B}$ grow symmetrically with $W^{2}$; at high $\left.W^{2},<\pi\right\rangle_{F}$ grows more than does $\left.<\pi\right\rangle_{B}$. In particular, at high $W^{2}$ the forward distribution has a larger tail at high $\pi$ than does the backward distribution. After correcting for instrumental effects, we find a net signal of $67 \pm 11$ more events at $\pi_{F}>3.0$ than we would expect from forward-backward symmetry.

In order to study the transverse shapes of hadron systems, we defined the variable planarity and studied its properties. Because planarity has no scale dependence, its distribution depends primarily on the multiplicities of the events being studied. However, a hard process underiying the production of final state hadrons may define a plane in which these hadrons are produced 
preferentially. Such a process also tends to give an event high $\pi$, leading to a correlation between $\pi$ and planarity. Our data show a correlation between high $\pi_{F}$ and planarity and also between high $\pi_{B}$ and planarity. While the high $\pi_{F}$, high planarity correlation is as expected for hard gluon bremsstrahlung, it is also consistent with other underlying hard processes that lead to events with high values of $\pi$. That such other processes may exist is demonstrated experimentally by the high $\pi_{B}$, high planarity signal, which should not result from hard gluon bremsstrahlung, and is demonstrated theoretically by the Monte Carlo calculations of the two-jet events where high $\pi$, high planarity events result from high $q_{T}$ quark-antiquark pairs bubbling up from the vacuum.

We studied the projected angular energy flow of several data samples and established the correspondence between the shape of a sampie's angular energy flow and the kinematic characteristics of that sample as measured by $\pi$ and planarity. While most of the data from our sample show a two-lobed angular energy flow, the high $\pi_{F}$, high planarity sample shows a three-lobed angular energy flow. This encourages the interpretation of the net high $\pi_{F}$, high planarity signal (above that expected from forward-backward symmetry) as a three-jet, hard gluon bremsstrahlung signal.

Interpreting our high $P_{T}$ signal as a hard gluon bremsstrahlung signal, we find $\left\langle\alpha_{s}\right\rangle=0.29 \pm 0.05 \pm 0.05$ for the three-jet events with $\pi_{F}>3.0$. The first error quoted is statistical and the second systematic. For three-jet events with $\pi_{F}>3.0$ our QCD Monte Cario also gives $\left\langle M_{9 g}^{2}\right\rangle \approx 25 \mathrm{GeV}^{2}, \quad\left\langle P_{T P}^{2}>\approx 10 \mathrm{GeV}^{2}, \quad\left\langle\hat{s}>\approx 100 \mathrm{GeV}^{2}, \quad\left\langle Q^{2}>\approx 45 \mathrm{GeV}^{2}, \quad\right.\right.\right.$ and $\left\langle W^{2}\right\rangle \approx 155 \mathrm{GeV}^{2}$. Our value for $\alpha$, accords well with that deduced from the three-jet rate observed in $e^{+} e^{-}$interactions at PETRA Interpreting our high $P_{T}$ signal as a three-jet signal, but without considering the underlying process, we observe a three-jet rate in high $W^{2}$ neutrino-nucleon interactions similar to that observed in $e^{+} e^{-}$interactions at PETRA.

Hadron transverse momentum increases with $W^{2}$, and does so more in the forward direction of the hadron system $\mathrm{CM}$ than in the backward. The high $\pi_{F}$, high planarity events show a three-lobed projected angular energy flow, which allows the natural interpretation of a hard underlying three-jet process. The QCD prediction of hard gluon bremsstrahlung provides a natural explanation of the extra growth of transverse momentum in the forward direction and provides a natural link between 
the net rate of three-jet events observed in this experiment and that observed in $e^{+} e^{-}$interactions at PETRA. The more simple Quark Parton Model is unable to explain our data, so we interpret our observations as evidence for the hard gluon bremsstrahlung process predicted by QCD. 


\section{References}

1. V. J. Stenger, in High Energy Physics - 1980, edited by L. Durand and L. G. Pondrom, AIP Conference Proceedings No. 68, American Institute of Physics, New York, 752 (1981).

H. H. Bingham, et al., in Proceedings of the 1981 International Conference on Neutrino Physics and Astrophysics, Maui, Hawaii, 148 (1981).

H. C. Ballagh et al., Phys. Rev. Lett. 47556 (1981).

2. G. M. Swider, Ph.D. Thesis, University of Washington, Seattle (1980).

3. R. J. Loveless et al., Phys. Lett. 78B, 505, (1978).

H. C. Ballagh et al., Phys. Lett. 89B, 423, (1980).

H. C. Ballagh et al., Phys. Rev. D 21569 (1980).

H. C. Ballagh et al., Phys. Rev. D 2424 (1981).

4. Eugene D. Commins, Weak Interactions, McGraw-Hill, New York (1973).

5. R. D. Field and R. P. Feynman, Nucl. Phys. B136, 1, (1978).

R. D. Field and R. P. Feynman, Phys. Rev. D15, 2590 (1977).

6. C. Day, private communication.

7. R. D. Field, in Quantum Flavordynamics, Quantum Chromodynamics, and Unified Theories, edited by K T. Mahanthappa and J. Randa, Plenum Press, New York, 221 (1980).

8. R. D. Peccei and R Ruckl, Nucl. Phys. B162 125, (1980).

9. M. Dechantsreiter et al., Z. Phys. C \&, 85, (1981).

Yu. L. Dokshitser etal., Phys. Lett. 78B, 290 (1978).

10. M. L Stevenson, in Proceedings of the Topical Conference on Neutrino Physics at Accelerators, Oxford, 1978, edited by A G. Michette and P. B. Renton, Rutherford Laboratory, England, 392 (1978).

11. J. Orthel, Ph.D. Thesis, University of Califoria, Berkeley, LBL-10035 (1979).

12. G. N. Taylor, private communication. The method used to calculate the beam spectrum for the quadrupole triplet train is similar to the method for the narrow band train described in $G$. N. 
Taylor, Ph.D. Thesis, University of Hawaii, Honolulu (1981).

13. R. D. Field and R. P. Feynman, Phys. Rev. D 152590 (1977).

14. T. H. Burnett, Quark Model Calculations, HEP.49, University of Washington, Seattle (1977).

15. R. N. Cahn, Phys. Lett. 28B, 269 (1978).

16. M. D. Sokoloff et al., Bull. Am. Phys. Soc. 27312 (1982).

17. H. Georgi and J. Sheiman, Phys. Rev. D 20111 (1979).

A. Mendez and T. Weiler, Phys. Lett. 83R 221 (1979).

18. C. Lo Basham et al., Phys. Lett. 858,297 (1979).

19. TASSO Collaboration, R. Brandelik et al., Phys. Lett. 24B, 437 (1980).

20. J. Lys, E 546 Studies - Neon Problems, internal memorandum, Lawrence Berkeley Laboratory, Berkeley (1982).

21. T Kitagaki et al., Phys. Lett. 97B, 325 (1980).

22. PLUTO Collaboration, L. Criegee and G. Knies, Physics Reports. 83 C. 151 (1982).

MARK-J Collaboration, D. P. Barber et al., Phys. Lett. 898, 139 (1980).

JADE Collaboration, W. Bartel et al., Phys. Lett. 21R, 142 (1980). 


\section{Appendix A: The Neutrino-Quark Cross Section}

We calculate the neutrino-quark charged current cross section

$$
\nu+q \rightarrow \mu^{-}+q^{\prime}
$$

starting with the well-known formula ${ }^{4}$

$$
d \sigma=\frac{(2 \pi)^{4} \delta^{4}\left(P_{\nu}+P_{q}-P_{\mu}-P_{q^{\prime}}\right)}{4\left[\left(P_{\nu} \cdot P_{q}\right)^{2}-\left(m_{\nu} m_{q}\right)^{2}\right]^{1 / 2}} \frac{d^{3} \vec{P}_{\mu} d^{3} \vec{P}_{q^{\prime}}}{(2 \pi)^{6}\left(2 E_{\mu}\right)\left(2 E_{q^{\prime}}\right)}|M|^{2},
$$

where the matrix element for the V-A interaction is ${ }^{4}$

$$
|M|^{2}=64 G^{2}\left(P_{p} \cdot P_{q}\right)\left(P_{\mu} \cdot P_{q^{\prime}}\right) .
$$

The neutrino is massless, and for our purposes the target quark may also be considered massless, so that

$$
d \sigma=\frac{4 G^{2}}{(2 \pi)^{2}} \frac{\left(E_{\mu} E_{q^{\prime}}-\vec{P}_{\mu} \cdot \vec{P}_{q^{\prime}}\right)}{E_{\mu} E_{q^{\prime}}} \delta^{4}\left(P_{\nu}+P_{q}-P_{\mu}-P_{q^{\prime}}\right) d^{3} \bar{P}_{\mu} d^{3} \vec{P}_{q^{\prime}}
$$

Integrating over $\vec{P}_{q^{\prime}}$ we eliminate the spatial part of the delta function to obtain

$$
d \sigma=\frac{4 G^{2}}{(2 \pi)^{2}} \frac{\left(E_{\mu} E_{q^{\prime}}-\vec{P}_{\mu} \cdot\left[\vec{P}_{\mu}+\vec{P}_{q}-\vec{P}_{\mu}\right]\right)}{E_{\mu} E_{q^{\prime}}} \delta\left(E_{\mu}+E_{q^{\prime}}-E_{y}-E_{q}\right) d^{3} \vec{P}_{\mu} .
$$

The cross section is a Lorentz invariant, so we may evaluate this expression in the neutrinoquark center-of-mass (CM) for ease of calculation. $\vec{P}_{p}^{C K}+\vec{P}_{q}^{C M}=0$, so

$$
d \sigma=\frac{4 G^{2}}{(2 \pi)^{2}}\left(1+\frac{\left|\vec{P}_{\mu}^{C M}\right|^{2}}{E_{\mu}^{C M} E_{q}^{C M}}\right)\left|\vec{P}_{\mu}^{C M}\right|^{2} \delta\left(E_{\mu}^{C M}+E_{q^{\prime}}^{C M}-2 E_{\nu}^{C M}\right) d\left|\vec{P}_{\mu}^{C M}\right| d \vec{\Omega}_{\mu}^{C M}
$$

where we have expanded $d^{3} \vec{P}_{\mu} C M$. Noting that the delta function may be considered a function of $\left|\vec{P}_{\mu}^{C M}\right|$

$$
\delta\left(\left[\left|\vec{P}_{\mu}^{C M}\right|^{2}+m_{\mu}^{2}\right]^{1 / 2}+\left[\left|\vec{P}_{\mu}^{C M}\right|^{2}+m_{q^{\prime}}^{2}\right]^{1 / 2}-2 E_{\nu}^{C M}\right),
$$

and approximating $m_{\mu}, m_{q^{\prime}} \ll\left|\vec{P}_{\mu}^{C M}\right|$, we integrate over $d\left|\vec{P}_{\mu}^{C M}\right|$ to get

$$
\frac{d \sigma}{d \vec{\Omega}_{\mu}^{C M}}=\frac{4 G^{2}}{(2 \pi)^{2}}\left(E_{\nu}^{C M}\right)^{2}
$$

In the neutrino-quark $\mathrm{CM}$ the cross section is flat in $\cos \theta_{\mu}$. As explained in Chapter II, section 2, this leads to a flat $y$ distribution.

Integrating over $d \vec{\Omega}_{\mu}^{C M}$ to get the total cross section,

$$
\sigma=\frac{4 G^{2}}{\pi}\left(E_{\nu}^{C M}\right)^{2}=\frac{G^{2}}{\pi}\left(2 E_{\nu}^{C M}\right)^{2}=\frac{G^{2}}{\pi} \hat{s}
$$


where $\dot{s}$ is the neutrino-quark invariant mass squared. As $\dot{s}$ is Lorentz invariant, we have the neutrino-quark charged current cross section in its most general form. 
Appendix B: The Sudakov Form Factor: Why and How

Order $\alpha_{s}$ corrections to the naive quark parton model due to real gluon emission give (semi-) inclusive cross sections that look like

$$
d \sigma=\int_{0}^{1} \frac{f(x)}{(1-x)+} d x
$$

where $f(x)$ is an analytic function and $\frac{1}{(1-x)_{+}}$is a functional. This functional is understood according to the prescription that $\frac{1}{(1-x)_{+}}=\frac{1}{(1-x)}$ for $x \neq 1$ and has a singularity at $x=1$ such that

$$
\int_{0}^{1} \frac{f(x)}{(1-x)+} d x=\int_{0}^{1} \frac{f(x)-f(1)}{(1-x)} d x .
$$

Thus it is well defined only when convoluted with a well behaved function.

Interpreting the integrand, $\frac{f(x)}{(1-x)_{+}}$, as the differential cross section, $\frac{d \sigma}{d x}$, with $\frac{1}{(1-x)_{+}}=\frac{1}{(1-x)}$ leads to problems as

$$
\int_{0}^{1-1} \frac{f(x)}{(1-x)_{+}} d x>\int_{0}^{1} \frac{f(x)}{(1-x)_{+}} d x
$$

for small, finite $\epsilon$. With the differential cross section interpretation this becomes

$$
\int_{0}^{1-4} \frac{d \sigma}{d x} d x>\int_{0}^{1} \frac{d \sigma}{d x} d x=d \sigma .
$$

The interpretation of $d \sigma$ as the three-jet cross section does not stand up; the whole cannot be less than the sum of the parts. (In fact $\int_{0}^{1-\frac{d}{d}} \frac{d \sigma}{d x} d x$ diverges as $\epsilon$ approaches 0 , apparently giving an infinite deep inelastic cross section).

The source of this problem in interpreting the integrand is in separating the inclusive cross section into two and three-jet components. The naive QPM diagram, figure II-3a, and its virtual gluon corrections, figure II-3b, have indistinguishable two parton final states (quark and diquark), while the two real gluon emission diagrams, figure II-3c, have indistinguishable three parton final states (quark, di-quark, and gluon). Thus the amplitudes of II-3a and II-3b are added separately 
from the amplitude of II-3c. The first combination gives what we call the two-jet cross section, and the second combination gives what we call the three-jet cross section. These cross sections both diverge in order $\alpha_{s}$, but their sum converges properly. The cancelling divergences tell us that separating the cross section into two- and three-jet final states is artificial in order $\alpha_{s}$, that the cases of soft gluon emission $\left(z_{q}-1\right)$ and of collinear gluon emission (with respect to the $\dot{Q}$ direction, $\left.x_{p} \rightarrow 1\right)$ are not distinct from the two-jet final states. In these limits $\frac{f(x)}{(1-x)_{+}}$must be modified before it can be interpreted as $\frac{d \sigma}{d x}$.

One method of doing this is to multiply the cross section at the parton transverse momentum, $P_{T P}$, by the Sudakov form factor ${ }^{9}$

$$
\exp \left(-\frac{2 \alpha_{s}}{3 \pi} \ln ^{2}\left[x_{T}^{2}\right]\right)
$$

where $x_{T}-\frac{\left(P_{T P}\right)_{\max }}{P_{T P}}$. The modified differential cross section becomes

$$
\left(\frac{d \sigma}{d x_{p} d z_{q}}\right)_{S}=\left(\frac{d \sigma}{d x_{p} d z_{q}}\right)_{1} \cdot \exp \left(-\frac{2 \alpha_{s}}{3 \pi} \ln ^{2}\left[\frac{1}{4 z_{q}\left(1-z_{q}\right)\left(1-x_{p}\right)}\right] .\right.
$$

For $x_{p}$ and $z_{q}$ both far from one the exponential approaches one. Thus the cross section for events which are clearly three-jet remains unchanged. As either $x_{p}$ or $z_{q}$ approaches one the damping effect of the exponential factor becomes important. This is illustrated for $x_{T}$, which is a convolution over $x_{p}$ and $z_{q}$, in figure B-1. $\frac{1}{\sigma_{0}} \frac{d \sigma}{d x_{T}}$ is plotted twice: first as given by equations II.27 - II.29, and then as modified by the Sudakov form factor.

Predictions that depend only on the higher $x_{T}$ region, where the two calculations of $\frac{d \sigma}{d x_{T}}$ agree, should be solid. Predictions which depend on the lower $x_{T}$ region are shaky. They depend on the evolution of narrow two-jet events into fat forward jet events into three-jet events.

In our Monte Carlo calculations of the QCD model, we have generated three-jet events only when the quark-gluon invariant mass, $M_{q g}$, is greater than $2 \mathrm{Gev}$ (see Chapter 4, section 1). This leaves most three-jet events with higher values of $x_{T}$ and the Sudakov form factor close to 1. Furth- 
ermore, it is the higher $M_{q g}$, higher $P_{T P}$ events which give the most effect on observable transverse momentum variables. $M_{q g}$ and $P_{T P}$ correlate strongly with $x_{T}$, so when one looks at the events which generate the high $P_{T}$ signals, the Sudakov form factor is not so important. In the smeared QCD Monte Carlo, for the three-jet events which are detected with $\pi_{F}>3.0$, the average value of the Sudakov form factor is about 0.85 .

The Sudakov form factor provides a straight-forward analytic method for damping the infra-red and collinear divergences of the three-jet cross section. The modified cross section is suitable for use in Monte Carlo programs and is not very different from the original cross section in that part of phase-space populated by events which contribute to the high $P_{T}$ signals. In predicting an observable three-jet rate, using the Sudakov form factor is much the same as using the undamped cross section with a smaller value of $\alpha_{s}$. When quoting $\left\langle\alpha_{s}\right\rangle$ from the QCD Monte Carlo (Chapters VI and VII) we have taken for $\alpha_{s}$ the product of the coupling constant in the Monte Carlo times the Sudakov form factor. This should correspond to the coupling constant of the undamped cross-section formula, equations II.27 - II.29. 


\section{Figure Captions}

Figure II-1: QPM Feynman diagram for neutrino-quark charged current scattering. We assume asymptotic freedom, that the target quark acts as a free particle for the duration of the weak interaction and the diquark as a spectator.

Figure II-2: Feynman diagram for the point-like four fermion V - A interaction. For $\nu_{\mu} q \rightarrow \mu^{-} q^{\prime}$, $P_{A}=P_{\mu}, P_{B}=P_{q}, P_{C}=P_{\mu}$, and $P_{D}=P_{q}$.

Figure II-3: QCD Feynman diagrams for neutrino-quark scattering, through $O\left(\alpha_{s}\right)$. Straight lines are fermions, as labeled. Wiggly lines are $W^{ \pm}$bosons. Curlyque lines are gluons. The diquark is not shown as it is assumed to be a spectator. a) The non-perturbative, QPM, diagram. b) $O\left(\alpha_{s}\right)$ virtual gluon diagrams. c) $O\left(\alpha_{s}\right)$ real gluon diagrams.

Figure II-4: Feynman diagrams for neutrino-gluon scattering to $O\left(\alpha_{s}\right)$.

Figure II-5: Feynman diagram of a higher order correction to the neutrino-quark cross section, inciuding a quark loop. The number of quark flavors, $N_{F}$ contributing such loops to the renormalization of quark and gluon lines is found in the formula for $\alpha_{s}$, equation II.32.

Figure III-1: The distribution of $\left(F R M S^{2} \cdot C R M S^{2}\right)$ for all tracks in events where all the tracks have nominally been reconstructed by the geometry programs.

Figure III-2: The WORST value of $\left(F R M S^{2}-C R M S^{2}\right)$ in each event where all the tracks have nominally been reconstructed by the geometry programs.

Figure III-3: The histogram is the distribution of $d p / p$ for charged hadron tracks in the data sampie, and the curve is the same distribution from the Monte Carlo.

Figure III-4: The fraction of charged hadron tracks removed from the data sample by the $d p / p>0.30$ cut is plotted as a function of track momentum. Also shown is the corresponding Monte Carlo curve.

Figure III-5: Feynman $x\left(x_{F}\right)$ distributions for charged tracks in the data sample, where $x_{F}$ is calculated using kinematic variables calculated for the event before the $x_{F}$ cut described in the text: (a) for negative tracks, (b) for positive tracks not identified as protons, (c) for positive tracks 
identified as protons.

Figure III-6: The histogram is the distribution of $d p / p$ for the muons in the data sample, and the curve is the same distribution from the Monte Cario.

Figure III-7: From the Monte Carlo, the average opening angle, as a function of hadron system invariant mass, between the visible hadron system, $\hat{q}_{v i s}$, and either the four momentum transfer direction, $\hat{q}$, (dashed curve) or the hadron system momentum direction, $\vec{P}_{N}+\vec{q}$, (solid curve).

Figure III-8: The distribution of $\Theta_{\text {OUT }}$ from our data sample, for events passing all cuts, with the Monte Carlo curve superposed. $\Theta_{\text {OUT }}$ is the angle between the visible hadron system and the lepton plane.

Figure III-9: The average value of $\Theta_{\text {OUT }}$, as a function of hadron system invariant mass, for events passing cuts, with the Monte Carlo curve superposed.

Figure III-10: A schematic drawing of the effect of Fermi motion on the determination of kinematic quantities describing an event. A neutrino which comes from the left would interact with a nucleon at rest to give muon and hadron system momenta $\vec{P}_{\mu}$ and $\vec{P}_{H}$. Were the neutrino to undergo the same scattering (in the neutrino-nucleon center-of-mass) with a nucleon moving up, the final muon and hadron system momenta would be $\vec{P}_{\mu^{\prime}}$ and $\vec{P}_{H^{\prime}}$.

Figure III-11: The resolution function for neutrino energy, $E_{v}$, for events passing cuts.

Figure III-12: The resolution function for hadron system energy, $E_{H}$, for events passing cuts.

Figure III-13: The resolution function for invariant four momentum transfer squared, $Q^{2}$, for events passing cuts.

Figure III-14: The resolution function for hadron system invariant mass, $W$, for events passing cuts. Figure III-15: The resolution function for the hadron system Lorentz boost factor $\gamma_{H}$, for events passing cuts.

Figure IV-1: The energy spectrum of neutrino events used as input for the Monte Carlos. This spectrum was calculated by $\mathrm{G}$. Taylor ${ }^{12}$ for the $200 \mathrm{GeV}$ tune of the Quadrupole Triplet focusing train at Fermilab and assumes the charged current cross section rises linearly with neutrino energy 
(hence $E_{\nu} d N / d E_{v}$ rather than $d N / d E_{v}$ as the ordinate).

Figure IV-2: The quark and antiquark momentum density functions, $\xi q(\xi)$, for the proton, from Field and Feynman ${ }^{13}$. The density functions for the neutron are found by charge conjugation, $\xi u_{n}(\xi)=\xi d_{p}(\xi)$, etc., where $\xi u_{n}(\xi)$ is the momentum density function for up quarks in a neutron and $\xi d_{p}(\xi)$ is the momentum density function for down quarks in a proton.

Figure IV-3: a) The $\left(x_{p}, z_{q}\right)$ scatter plot for three-jet events generated by the QCD Monte Carlo. The quark-gluon invariant mass must be greater than $2 \mathrm{GeV}$, and the cross section of Eqs. II-27 through II -29 has been multiplied by the Sudakov form factor described in Appendix B. b) The $x_{p}$ distribution, integrating over $z_{q}$. c) The $z_{q}$ distribution, integrating over $x_{p}$.

Figure IV-4: The quark-gluon invariant mass distribution for three-jet events generated by the Monte Carlo a) plotted on a linear scale, b) plotted on a semi-log scale.

Figure IV-5: The parton transverse momentum distribution for three-jet events generated by the QCD Monte Cario a) plotted on a linear scale, b) plotted on a semi-log scale.

Figure IV-6: The quark azimuthal angle distribution for three-jet events generated by the QCD Monte Carlo. The angle is measured about the momentum transfer direction such that the azimuthal angle of the muon is 0 and the azimuthal angle of $\vec{P}_{\nu} x \vec{P}_{\mu}$ is $+\frac{\pi}{2}$.

Figure IV-7: The distribution of the $\cos \phi_{q}$ asymmetry parameter, $B / A=d \sigma_{q}^{(1)} / d \sigma_{q}^{(0)}$. See equations II.32 and IV.2.

Figure IV.8: The distribution of the $\cos 2 \phi_{q}$ asymmetry parameter, $C / A=d \sigma_{q}^{(2)} / d \sigma_{q}^{(0)}$. See equations II.32 and TV.2.

Figure IV-9: From Field and Feynman ', "Illustration of the 'hierarchy' structure of the final mesons produced when a quark of type 'a' fragments into hadrons. New quark pairs bīb, cé, etc., are produced and 'primary' mesons are formed. The 'primary' meson b̄a that contains the original quark is said to have 'rank' one and primary meson īb rank two, etc. Finally, some of the primary mesons decay and we assign all the decay products to have the rank of the parent. The order in 'hierarchy' is not the same as order in momentum or rapidity." 
Figure IV-10: $\left\langle P_{T}^{2}\right\rangle$ as a function of $W^{2}$ for observable hadrons in the unmodified TUBES two-jet model.

Figure V-1: a) The quark-weak current opening angle distribution for all three-jet events generated in the QCD Monte Carlo. b) As in (a), but plotted separately for three ranges of quark-giuon invariant mass. c) As in (a), but plotted separately for three ranges of parton transverse momentum. d) The gluon-weak current opening angle distribution for all three-jet events generated in the QCD Monte Carlo. e) As in (d), but plotted separately for three ranges of quark-giuon invariant mass. f) As in (d), but plotted separately for three ranges of parton transverse momentum.

Figure V-2: Average value of parton transverse momentum squared as a function of hadron system invariant mass. The solid curve is the average when summing over three-jet events only. The dashed curve is the average when also summing over the two-jet events where $P_{T P}=0$.

Figure V-3: Average value of quarks' longitudinal momentum fraction as a function of hadron system invariant mass. The solid curve is for three-jet events only, and the dashed curve is for all events. For three-jet events, the sharp rise at low $W$ is an artifact of demanding that the quarkgluon invariant mass be greater than $2 \mathrm{GeV}$, see Eq. II-17.

Figure V-4: Pristine Monte Carlo calculations of average transverse momentum squared of charged hadrons, as a function of $x_{F}$, for events with $W^{2}>50 \mathrm{GeV}^{2}$. The QPM curve is forward-backward symmetric. The QCD curve is higher for $x_{F}>0$, but coincides with the QPM curve for $x_{F}<0$. The difference between the two models, $\delta P_{T}^{2}$, is also plotted.

Figure V-5: Pristine QPM calculation of $\left\langle P_{T}^{2}\right\rangle$, as a function of hadron system invariant mass, for all charged hadrons.

Figure V-6: Pristine $Q P M$ and $Q C D$ calculations of $\left\langle P_{T}^{2}\right\rangle_{F},\left\langle P_{T}^{2}\right\rangle_{B}$, and $\Delta P_{T}^{2}$, as functions of hadron system invariant mass. a) for $\left|x_{F}\right|>0.00$, b) for $\left|x_{F}\right|>0.05$, c) for $\left|x_{F}\right|>0.10$, d) for $\left|x_{F}\right|>0.20$.

Figure V-7: The Feynman $x$ distribution for charged hadrons after all cuts. The QCD and QPM Monte Carlo are more sharply peaked than the data, but for $x_{F}>0.05$, where most of the analysis 
will be done, the differerce is small.

Figure V-8: Measured $\left\langle P_{T}^{2}\right\rangle_{F},\left\langle P_{T}^{2}\right\rangle_{B}$, and $\Delta P_{T}^{2}$ as functions of hadron system invariant mass, with the smeared QPM and QCD curves also plotted. a) for $\left|x_{F}\right|>0.00$, b) for $\left.\left|x_{F}\right|>0.05, \mathrm{c}\right)$ for $\left|x_{F}\right|>0.10$, d) for $\left|x_{F}\right|>0.20$.

Figure V-9: Measured $\left\langle P_{Q U T}^{2}\right\rangle_{F},\left\langle P_{Q U T}^{2}\right\rangle_{B}$, and $\Delta P_{Q U T}^{2}$ for $x_{F}>0.05$, as functions of hadron system invariant mass, with smeared QPM and QCD curves also plotted.

Figure V-10: a) Ratio of $\left\langle P_{T}^{2}\right\rangle_{F}$ to $\left\langle P_{\alpha U T}^{2}\right\rangle_{F}$ for $\left|x_{F}\right|>0.05$ as a function of hadron system invariant mass. The QPM and QCD Monte Carios give the same curve, which is plotted with the data. b) Ratio of $\left\langle P_{T}^{2}\right\rangle_{B}$ to $\left\langle P_{Q C T}^{2}\right\rangle_{B}$ for $\left|x_{F}\right|>0.05$ as a function of the hadron system invariant mass. Again, the $Q C D$ and QPM Monte Carlos give the same curve, which is plotted with the data.

Figure V-11: a) $\pi_{F}$ for all events, where the sum defining $\pi_{F}$ includes all charged tracks with $x_{F}>$ 0.00 . b) $\pi_{B}$ for all events, where the sum defining $\pi_{B}$ includes all charged tracks with $x_{F}<0.00$.

Figure V-12: Measured $\left\langle\pi_{F}\right\rangle,\left\langle\pi_{B}\right\rangle$, and $\langle\Delta \pi\rangle$, as functions of hadron system invariant mass, with smeared QPM and QCD curves. also plotted. a) for $\left|x_{F}\right|>0.00$, b) for $\left|x_{F}\right|>0.05$, c) for $\left|x_{F}\right|>0.10$, d) for $\left|x_{F}\right|>0.20$.

Figure V-13: a) The pristine and smeared Monte Carlo $\pi_{F}$ distributions for the QPM and QCD modeis for events with $W^{2}>50 \mathrm{GeV}^{2}$. b) The pristine and smeared Monte Carlo $\pi_{B}$ distributions for the QPM and QCD models for events with $W^{2}>50 \mathrm{GeV}^{2}$. There is no observable difference between the QPM and QCD calculations for $\pi_{B}$, so only one pristine and one smeared curve are plotted.

Figure V-14: The ratio of $\frac{1}{N} \frac{d N}{d \pi_{F(B)}}$ observed with instrumental effects included to that with instrumental effects excluded. The difference between the QCD and QPM curves for the forward direction stems from the different shapes of the true $\pi_{F}$ distributions in the model. See the text for a fuller explanation. 
Figure V-15: a) Observed $\pi_{F}$ and $\pi_{B}$ rates for $W^{2}<50 \mathrm{GeV}^{2}$ with the smeared QCD curves superposed. b) Observed $\pi_{F}$ and $\pi_{B}$ rates for $W^{2}>50 \mathrm{GeV}^{2}$ with the smeared QCD curves superposed. Note that although the curves fall somewhat below the data, the difference comes out about right. Figure V-16: The net rate of high $\pi_{F}$ events as a function of $\pi_{F}$, for $W^{2}>50 \mathrm{GeV}^{2}$. For each range of $\pi$, the number of $\pi_{B}$ events observed in the range was multiplied by $\left(\epsilon_{F} / \epsilon_{B}\right)$ from figure $V$ 14 and by the ratio of the number of events with $N_{F}$ not equal 0 to that with $N_{B}$ not equal 0 to get the symmetric background. $\epsilon_{F}$ was taken from the QPM curve in figure V-14 to be more conservative in establishing a non-zero signal.

Figure V-16: a) The $\left(\pi_{F}, \pi_{B}\right)$ scatter plot for $W^{2}<50 \mathrm{GeV}^{2}$ events. b) The $\left(\pi_{F}, \pi_{B}\right)$ scatter plot for $W^{2}>50 \mathrm{GeV}^{2}$ events.

Figure V-18: $<\pi_{B}>$ as a function of $\pi_{F}$ for low $W^{2}$ and high $W^{2}$, with Monte Carlo curves also plotted. The results of the QPM and QCD calculations were indistinguishable, so only one curve is plotted for each of low $W^{2}$ and high $W^{2}$.

Figure V-19: $\left\langle\pi_{F}\right\rangle$ as a function of $\pi_{B}$ for low $W^{2}$ and high $W^{2}$, with Monte Carlo curves also plotted. The QPM and QCD calculations differ here as $\pi_{F}$ tends to be higher in the QCD Monte Carlo than in the QPM Monte Carlo, particulariy at high $W^{2}$.

Figure V-20: Average planarity as a function of charge multiplicity. The Monte Carlo calculations for the QPM and QCD models, for low $W^{2}$ and high $W^{2}$, all gave the same result, which is plotted with the data. The small difference between the data and the Monte Carlos is probably due to the somewhat higher $\gamma$ multiplicity in the Monte Carlos.

Figure V-21: Charge multiplicity (after cuts) as a function of hadron system invariant mass. The QPM and QCD Monte Carlo curves are also plotted. The differences between theses curves result primarily from TUBES treatment of central meson production (which also gives the wrong $x_{F}$ distribution near $x_{F}=0$, see figures V-7 and V-32), and is not fundamental to the underlying QPM and QCD modeis. Note also that part of the charge multiplicity in the data, but not in the Monte Carlos, is due to nuclear debris and re-interactions. 
Figure V-22: a) Planarity distribution for events with $W^{2}<50 \mathrm{GeV}^{2}$. The QPM and QCD curves differ slightly due to the different multiplicity distributions in the models. See also figures V-20 and V-21. b) As in (a), but for $W^{2}>50 \mathrm{GeV}^{2}$.

Figure V-23: The $\left(\pi_{F}, P\right)$ scatter plot for $W^{2}>50 \mathrm{GeV}^{2}$ events.

Figure V-24: a) Charge multiplicity distribution for $W^{2}>50 \mathrm{GeV}^{2}$ with $\pi_{F}<2.0$. b) Charge multiplicity distribution for $W^{2}>50 \mathrm{Gev}$ events with $\pi_{F}>3.0$.

Figure V-25: Planarity distribution for events with $W^{2}>50 \mathrm{GeV}^{2}$ and $\pi_{F}>3.0$. Monte Carlo QPM and QCD histograms (normalized to the same area as the data) are also plotted.

Figure V-26: The $\left(\pi_{B}, P\right)$ scatter plot for $W^{2}>50 \mathrm{GeV}^{2}$ events.

Figure V-27: a) The $\left(\pi_{F}, P\right)$ scatter plot for $W^{2}<50 \mathrm{GeV}^{2}$ events. b) The $\left(\pi_{B}, P\right)$ scatter plot for $W^{2}<50 \mathrm{GeV}^{2}$ events.

Figure V-28: a) Average pianarity as a function of $\pi_{F}$, for low $W^{2}$ and high $W^{2}$ separately. For low $W^{2}$ the QPM and QCD Monte Carlo calculations agree, so only one curve is plotted. b) Average planarity as a function of $\pi_{B}$, as was done in (a) for $\pi_{F}$.

Figure V-29: From the smeared QPM Monte Carlo for events with $W^{2}>50 \mathrm{GeV}^{2}$, the highest value per event of $k_{T}$, the transverse momentum of quark-antiquark pairs bubbiing up from the vacuum. The $k_{T}$ spectrum for events with $\pi_{F}>3.0$ has been multiplied by 5 and shaded in.

Figure V-30: a) The $\left(\pi_{F}, P\right)$ scatter plot for $W^{2}>50 \mathrm{GeV}^{2}$ events in the smeared Monte Carlo of the modified two-jet model described in the text. There are about 2700 entries in this plot, which is somewhat more than in the corresponding scatter plot of the data, figure V-23. b) The projection of (a) onto the $\pi_{F}$ axis. c) The projection of (a) onto the planarity axis.

Figure V-31: a) The projected angular energy flow, onto the $\left(\dot{Q}_{v i r}, \hat{u}_{1}\right)$ plane, for all events with $W^{2}>50 \mathrm{GeV}^{2}$, with the $\mathrm{QPM}$ and $\mathrm{QCD}$ curves superposed. (normalized to the same number of events). The inset is a poiar plot of the data only, which illustrates the two-jet interpretation. b) The projected angular energy flow onto the $\left(\dot{Q}_{v i s}, \hat{u}_{2}\right)$ plane for all events with $W^{2}>50 \mathrm{GeV}$, with the QPM and QCD curves superposed. The inset is a polar plot of the data only. 
Figure V-32: The Feynman $x$ distribution for charged hadrons in $W^{2}>50 \mathrm{GeV}^{2}$ events. The $\mathrm{QCD}$ and QPM Monte Carlo curves are also plotted.

Figure V-33: $\left\langle P_{T}^{2}\right\rangle$ as a function of Feynman $x$, for $W^{2}>50 \mathrm{GeV}$ events. The QCD and QPM Monte Carlo curves are aiso plotted. For comparison, the pristine versions of the Monte Carlo curves are found in figure V-4.

Figure V-34: a) The projected angular energy flow, onto the $\left(\dot{Q}_{\nu i s}, \dot{u}_{1}\right)$ plane, for $W^{2}>50 \mathrm{GeV}^{2}$ events with $\pi_{F}>3.0$ and $P>0.5$, with the $\mathrm{QPM}$ and $Q C D$ curves also plotted (normalized to the same number of events). The inset is a polar plot of the data only, which illustrates the three-jet interpretation of this sample. b) The projected angular energy flow onto the $\left(\hat{Q}_{v i s}, \dot{u}_{2}\right)$ plane, for $W^{2}>50 \mathrm{GeV}^{2}$ events with $\pi_{F}>3.0$ and $P>0.5$, with the QPM and QCD Monte Carlo curves also plotted. The inset is a polar plot of the data only. Nota Bene: The Monte Carlo curves were drawn by hand through plots of Monte Carlo data with error bars similar to those seen in the plots of the real data. The QCD Monte Carlo had about ten times the statistics of the data, hence smaller error bars. The QPM Monte Carlo had only about the same statistics as the data. (Many more QPM events were generated than exist in the real data sample, but there are disproportionately fewer at high $\pi_{F}$, high planarity.) Although the QPM and QCD curves look different, within statistics the QPM and QCD curves are indistinguishable. the QCD curve describes the QPM Monte Cario data as well as it does the real data.

Figure VI-1: Quark-gluon invariant mass distribution from the QCD Monte Carlo for smeared events with $W^{2}>50 \mathrm{GeV}^{2}$ and passing cuts. The spectrum for events with $\pi_{F}>3.0$ and $P>0.6$ as well is multiplied by five and shaded in.

Figure VI-2: The fraction of three-jet events with $\pi_{F}>3.0$ and $P>0.6$ in the smeared $Q C D$ Monte Carlo as a function of quark-gluon invariant mass.

Figure VI-3: Parton transverse momentum distribution from the QCD Monte Cario for smeared events with $W^{2}>50 \mathrm{GeV}^{2}$ and passing cuts. The spectrum for events with $\pi_{F}>3.0$ and $P>0.6$ as well is multiplied by five and shaded in. 
Figure VI-4: The fraction of three-jet events with $\pi_{F}>3.0$ and $P>0.6$ in the smeared $Q C D$ Monte Carlo, as a function of parton transverse momentum.

Figure VI-5: The $W^{2}$ spectra of the two-jet and of the three-jet events passing cuts in the pristine QCD Monte Cario, for $W^{2}>50 G e V^{2}$.

Figure VI-6: a) The $W^{2}$ spectra of the three-jet events passing cuts in the pristine QCD Monte Carlo for three ranges of quark-gluon invariant mass. b) The $W^{2}$ spectra of the three-jet events passing cuts in the pristine $Q C D$ Monte Cario for three ranges of parton transverse momentum.

Figure VI-7: The $E$, spectra of the two-jet events and of the three-jet events passing cuts in the pristine Monte Carlo, for $W^{2}>50 \mathrm{GeV}^{2}$.

Figure VI-8: a) The $E$, spectra of the three-jet events in the pristine Monte Carlo for three ranges

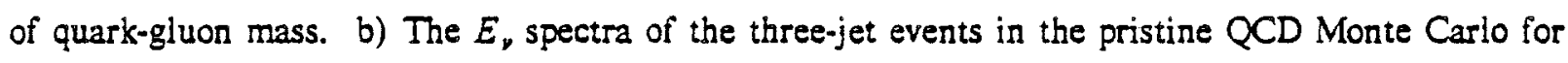
three ranges of parton transverse momentum.

Figure VI-9: The $\nu$ spectra of the two-jet and three-jet events passing cuts in the pristine Monte Carlo, for $W^{2}>50 \mathrm{GeV}^{2}$.

Figure VI-10: a) The $\nu$ spectra of the three-jet events in the pristine Monte Carlo for three ranges of quark-gluon invariant mass. b) The $\nu$ spectra of the three-jet events in the pristine Monte Carlo for three ranges of parton transverse momentum.

Figure VI-11: The $Q^{2}$ spectra of the two-jet and of the three-jet events passing cuts in the pristine QCD Monte Carlo, for $W^{2}>50 \mathrm{GeV}^{2}$.

Figure VI-12: a) The $Q^{2}$ spectra of the three-jet events in the pristine QCD Monte Cario for three ranges of quark-gluon invariant mass. b) The $Q^{2}$ spectra of the three-jet events in the pristine $Q C D$ Monte Carlo for three ranges of parton transverse momentum.

Figure VI-13: The $y$ distributions of the two-jet events and of the three-jet events passing cuts in the pristine QCD Monte Carlo, for $W^{2}>50 \mathrm{GeV}^{2}$.

Figure VI-14: a) The $y$ distributions of the three-jet events in the pristine QCD Monte Cario for three ranges of quark-gluon invariant mass. b) The $y$ distributions of the three-jet events in the 
pristine QCD Monte Carlo for three ranges of parton transverse momentum.

Figure VI-15: The Bjorken $x$ distributions of the two-jet and three-jet events passing cuts in the pristine $\mathrm{QCD}$ Monte Carlo, for $W^{2}>50 \mathrm{GeV}^{2}$.

Figure VI-16: a) The Bjorken $x$ distributions of the three-jet events in the pristine QCD Monte Carlo for three ranges of quark-giuon invariant mass. b) The Bjorken $x$ distributions of the threejet events in the pristine QCD Monte Carlo for three ranges of parton transverse momentum.

Figure VI-17: The quark longitudinal momentum fraction distributions of the two-jet and of the three-jet events passing cuts in the pristine $\mathrm{QCD}$ Monte Cario, for $W^{2}>50 \mathrm{GeV}^{2}$.

Figure VI-18: a) The quark longitudinal momentum fraction distributions of the three-jet events in the pristine QCD Monte Carlo for three ranges of quark-gluon invariant mass. b) The quark longitudinal momentum fraction distributions of the three-jet events in the pristine QCD Monte Cario for three ranges of parton transverse momentum.

Figure VI-19: The $\dot{s}$ spectra of the two-jet and of the three-jet events passing cuts in the pristine QCD Monte Cario, for $W^{2}>50 \mathrm{GeV}^{2}$.

Figure VI-20: a) The $s$ spectra of the three-jet events in the QCD Monte Cario for three ranges of quark-gluon invariant mass. b) The $\hat{s}$ spectra of the three-jet events in the QCD Monte Carlo for three ranges of parton transverse momentum.

Figure VI-21: $\left\langle P_{T}^{2}\right\rangle$ of charged tracks with $\left.x_{F}\right\rangle 0.05$ as a function of hadron system invariant mass, from the pristine QCD Monte Cario, piotted separately for two-jet events and for three-jet events in three ranges of quark-gluon invariant mass.

Figure V1-22: As in figure V-21, but from the smeared QCD Monte Carlo.

Figure VI-23: The $\pi_{F}$ distributions from the pristine QCD Monte Carlo for two-jet events and for three-jet events (separately for three ranges of $M_{q g}$ ) that have $W^{2}>50 \mathrm{GeV}^{2}$ and pass cuts.

Figure VI-24: As in figure VI-23, but from the smeared QCD Monte Carlo.

Figure VI-25: The planarity distributions from the pristine $Q C D$ Monte Carlo for two-jet events and for three-jet events (separately for three ranges of $M_{q g}$ ) that have $W^{2}>50 \mathrm{GeV}^{2}$ and pass cuts. 
Figure VI-26: As in figure VI-25, but from the smeared QCD Monte Carlo.

Figure B-1: $\frac{1}{\sigma_{0}} \frac{d \sigma}{d X_{T}}$ calculated using a valence quark distribution proportional to $\frac{(1-x)^{3}}{x}$ and a running coupling constant whose argument is the invariant mass of the final state quark-giuon pair. The dashed curve is the $O\left(\alpha_{s}\right)$ cross section from equation II.32. The solid curve is the dashed curve multiplied by the Sudakov form factor discussed in Appendix B. 


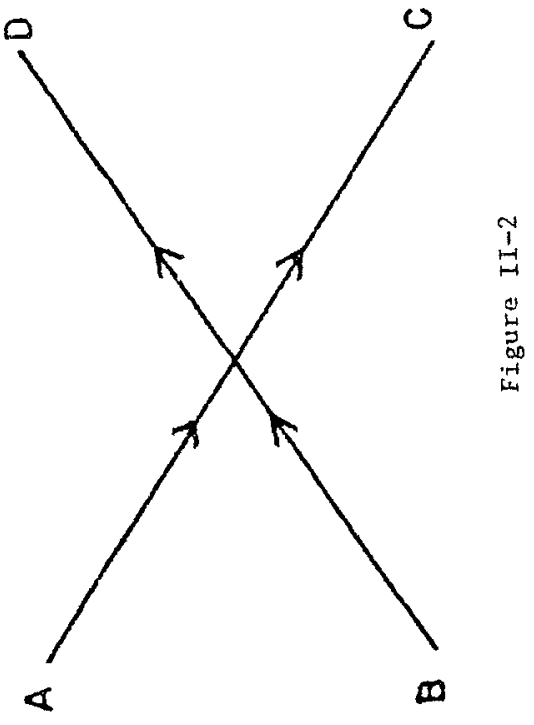

$\stackrel{1}{\infty}$

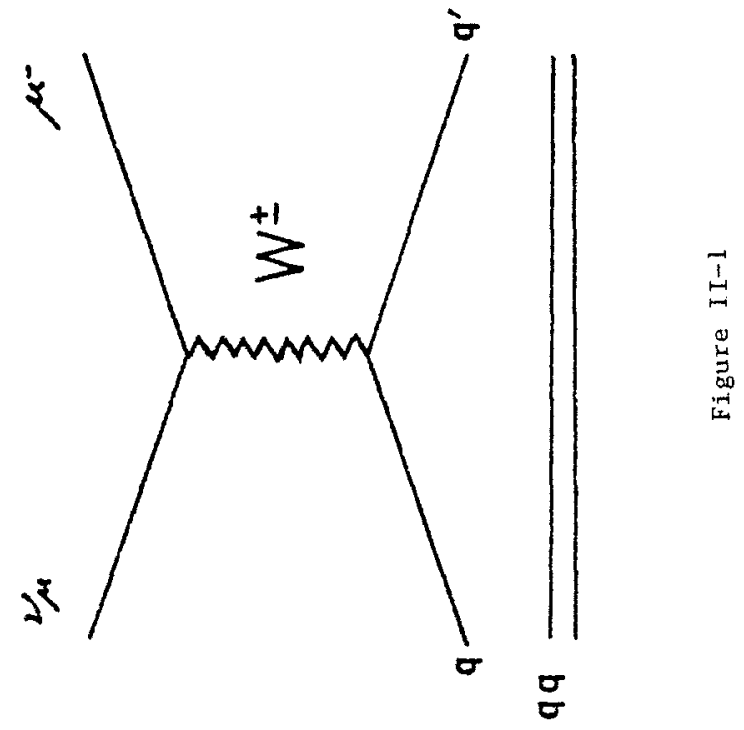




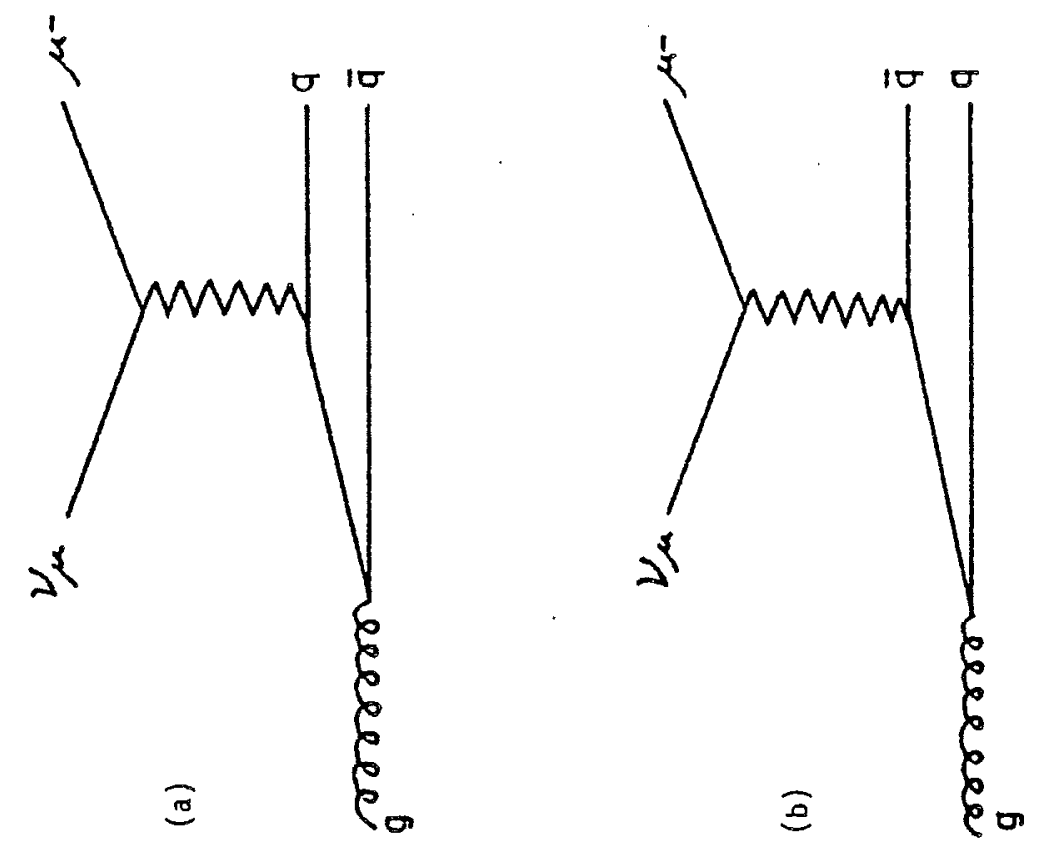

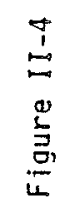

1
1
1
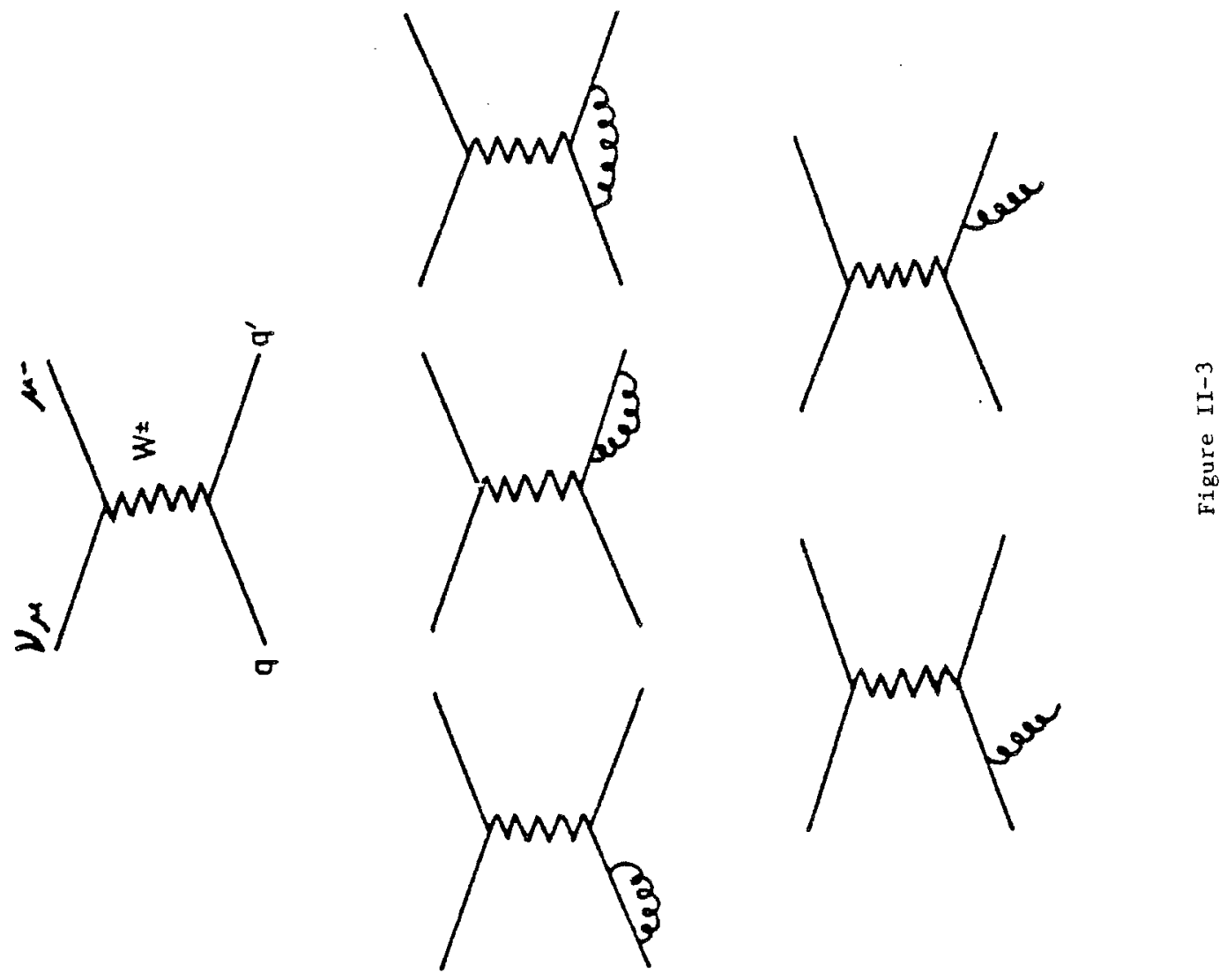

J

0

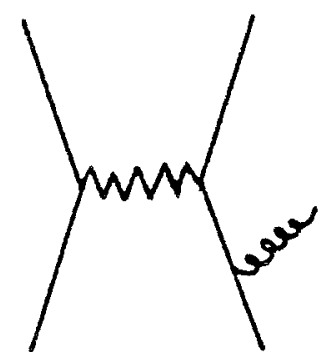

0 


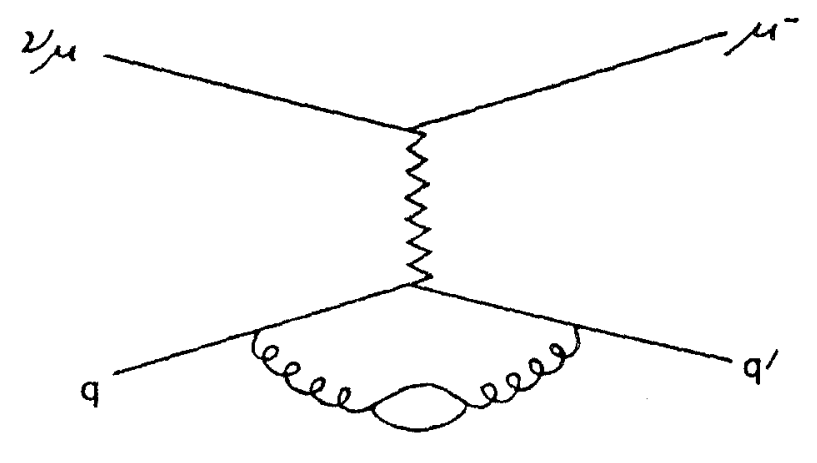

Figure II-5

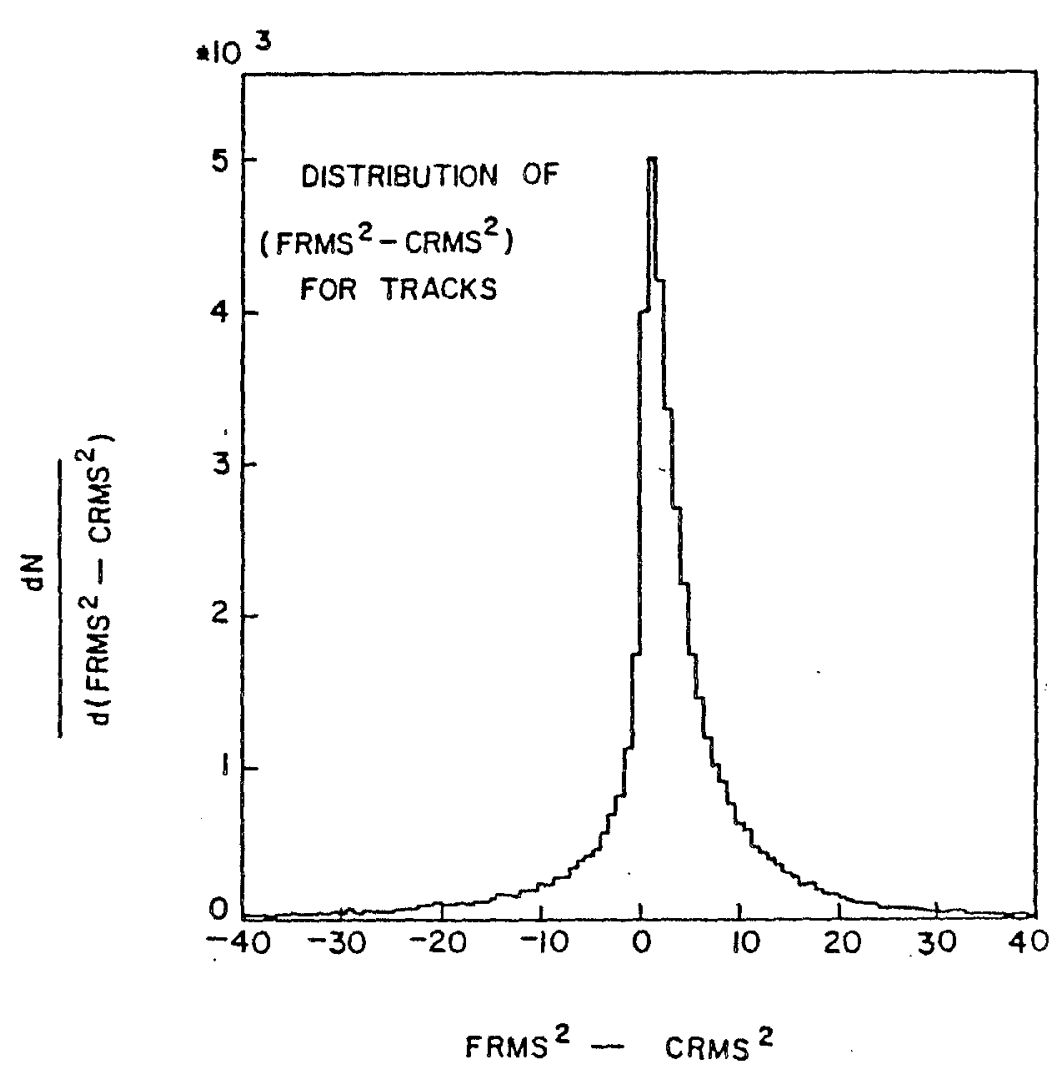

Figure III-I 
- 90 -

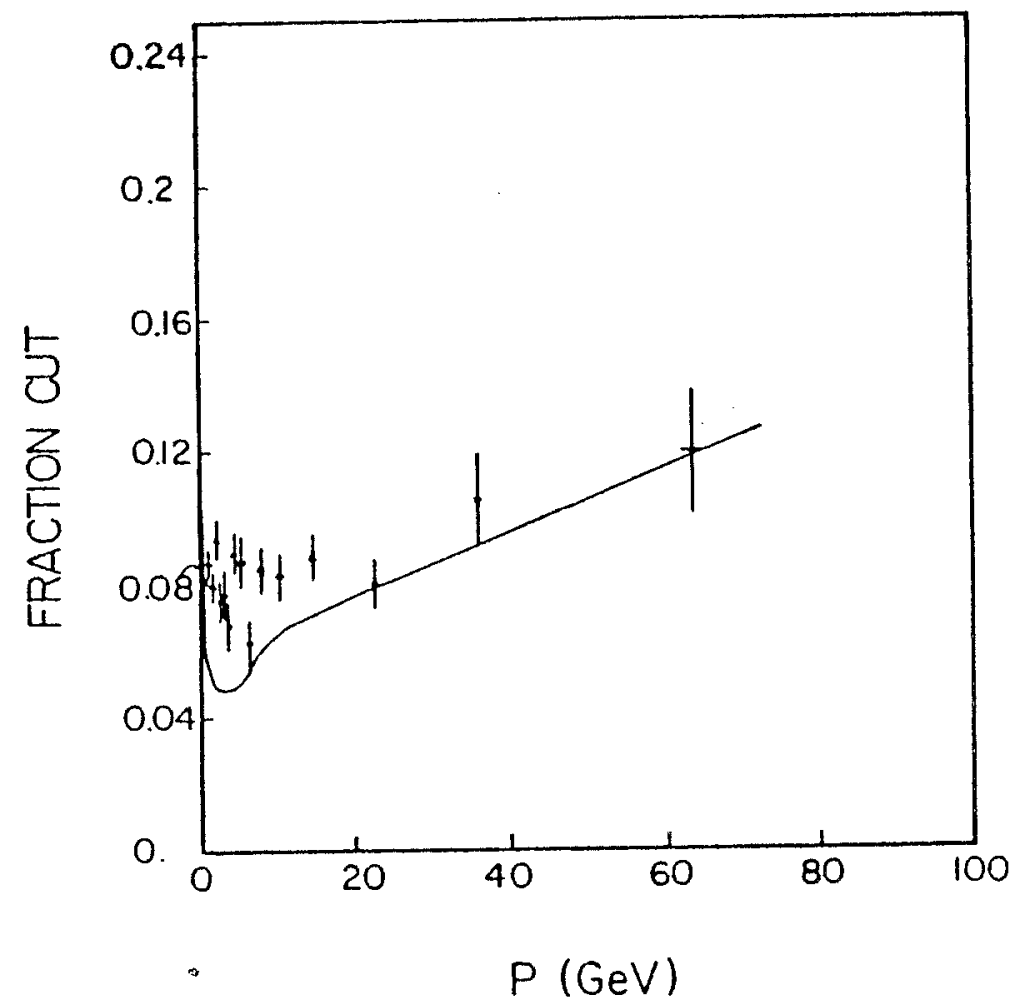

Figure III-4

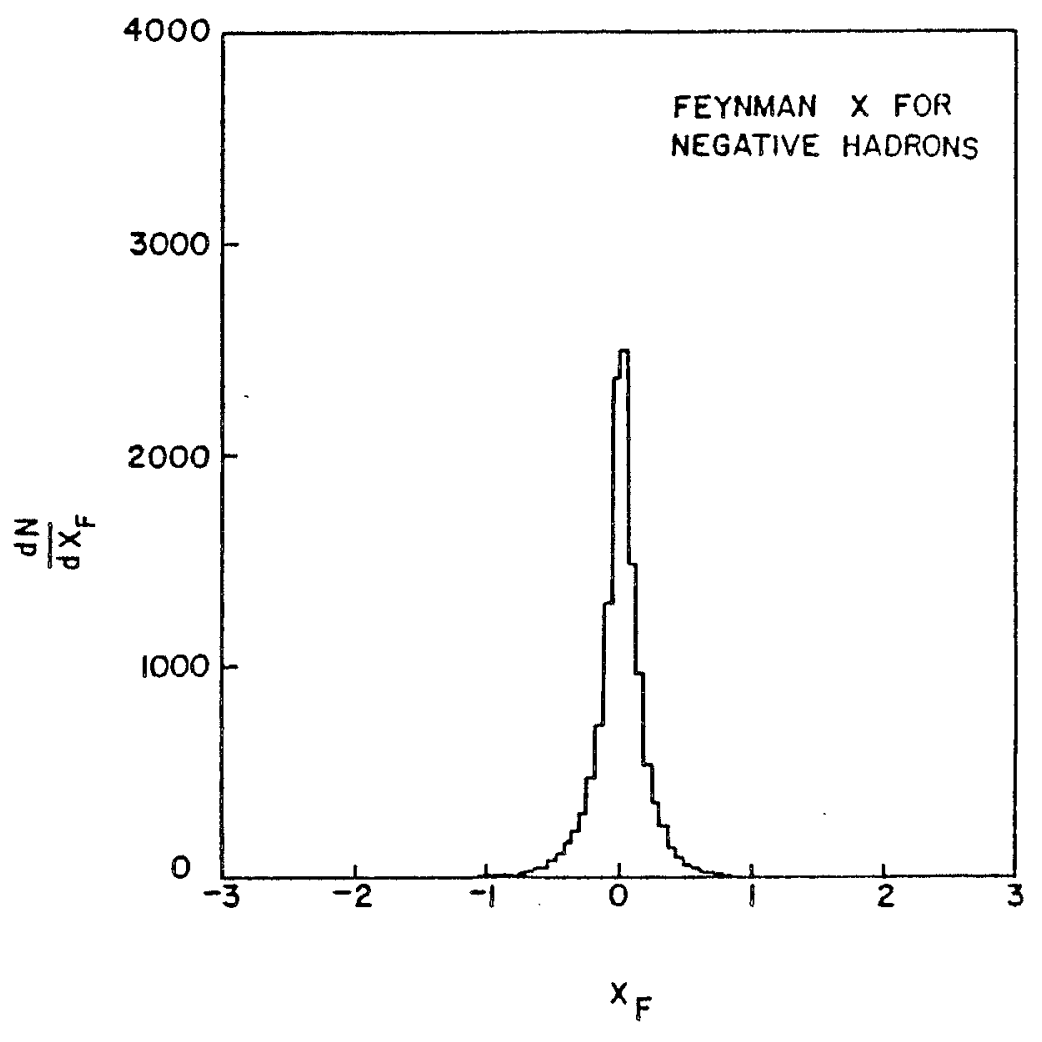

Figure III-5(a) 


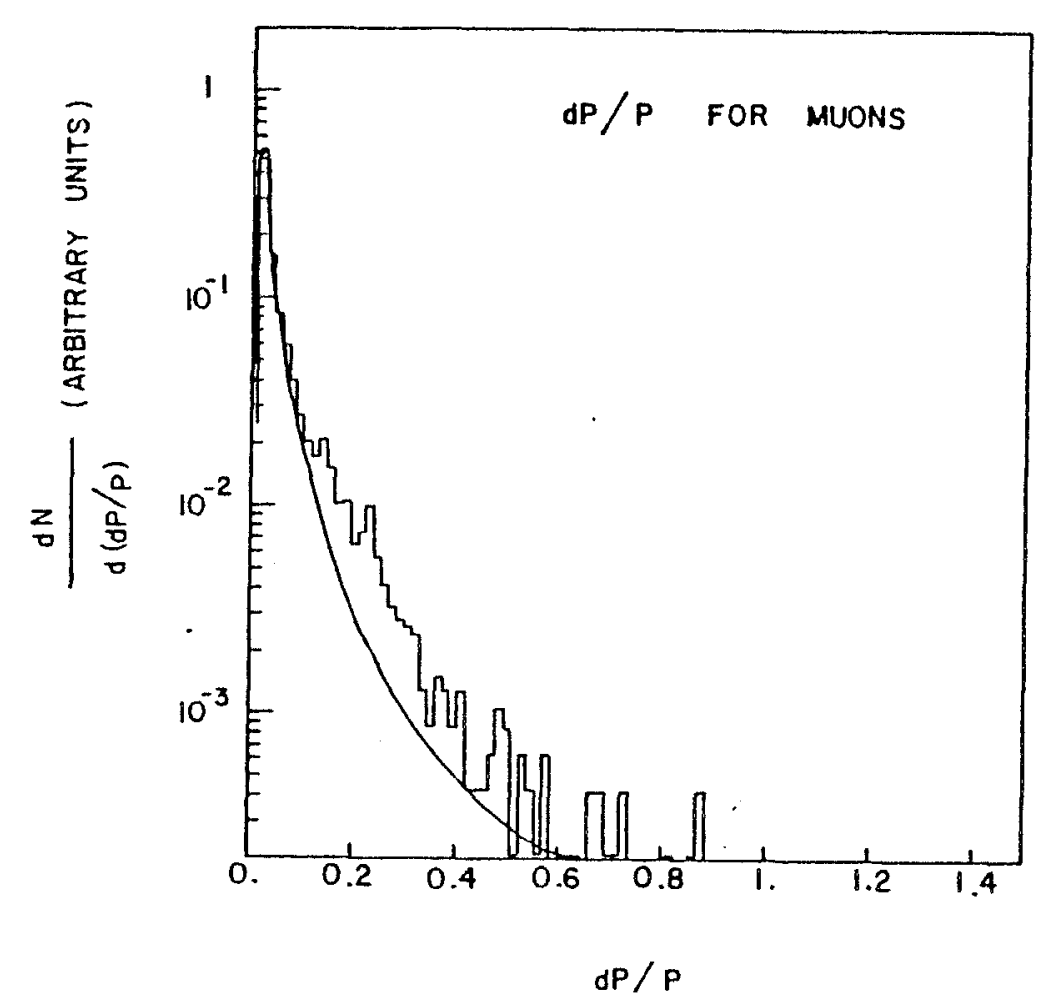

Figure III-6

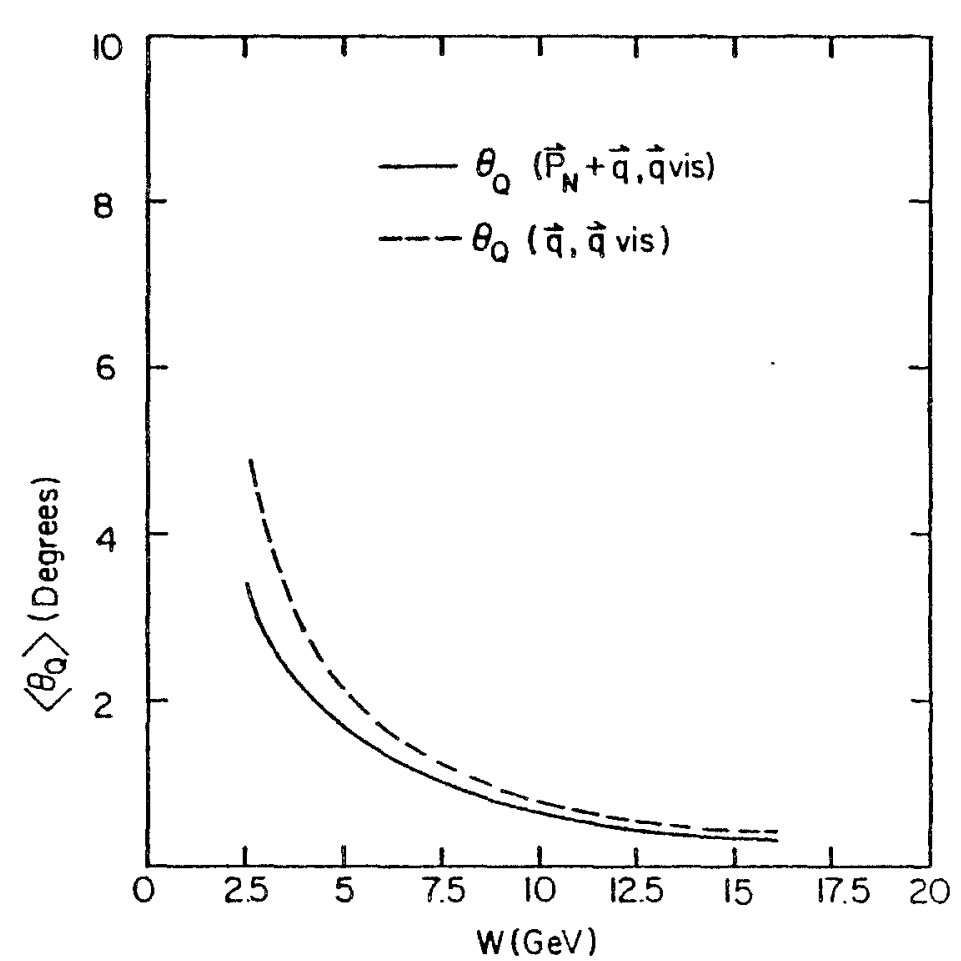

Figure III-7 

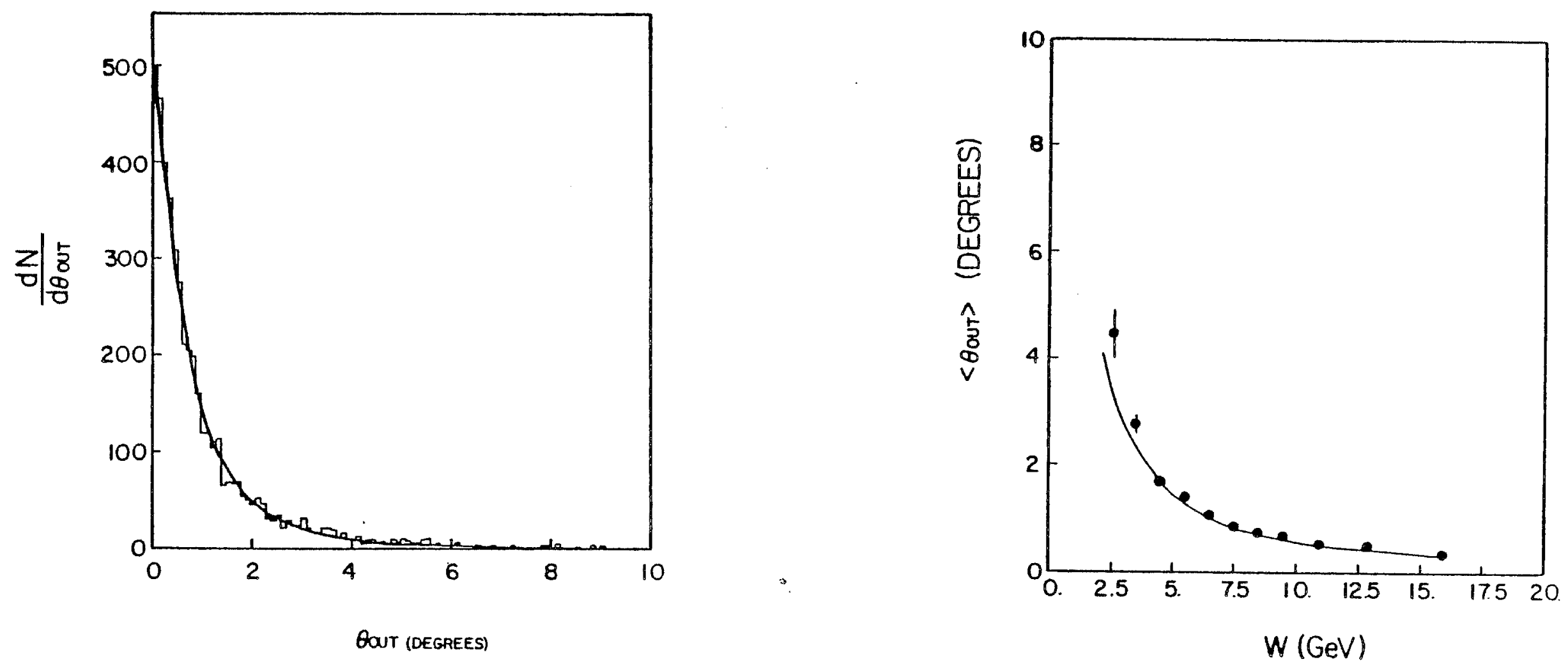

Fiqure III-8

Figure III-9 


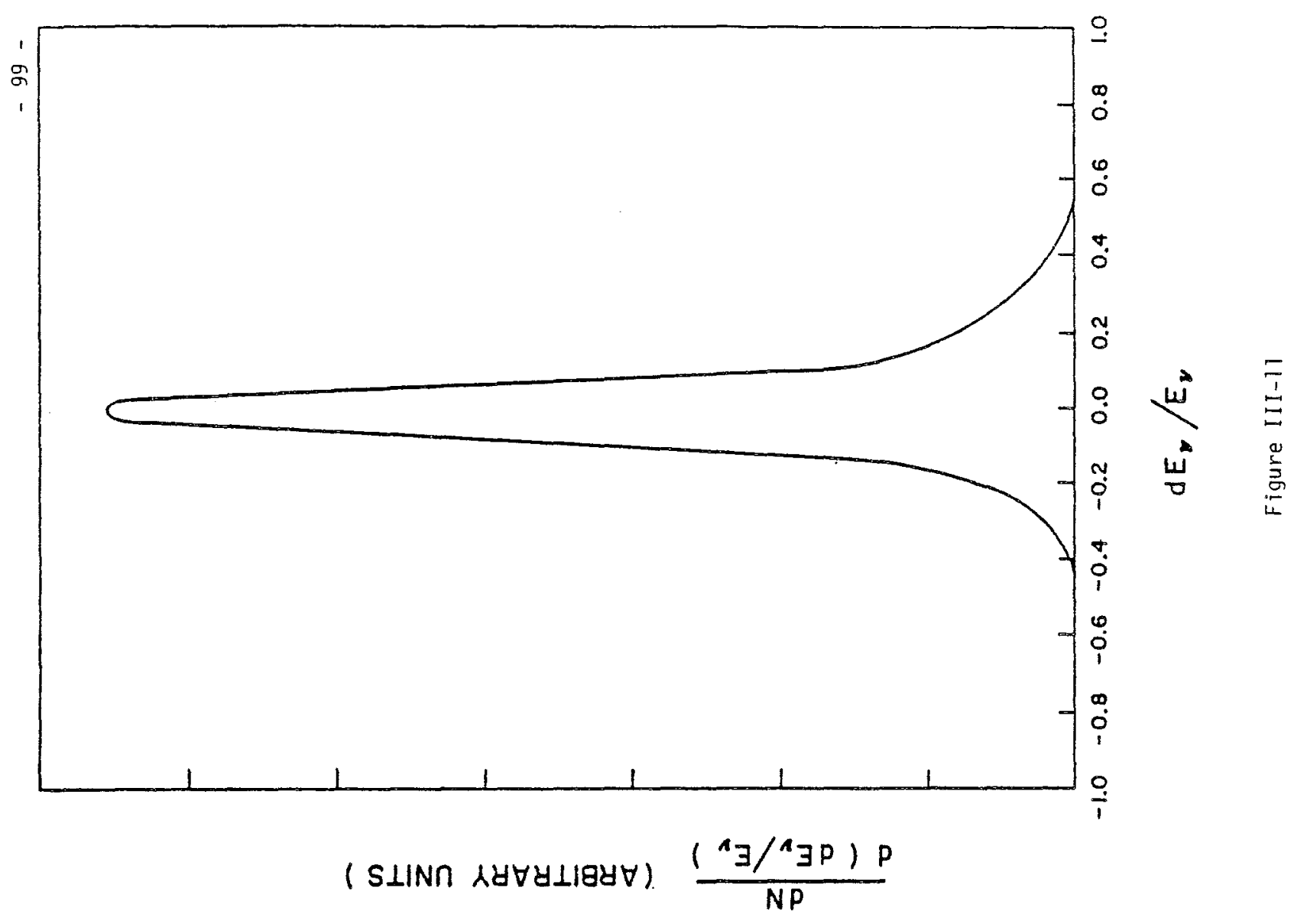

1
o
1

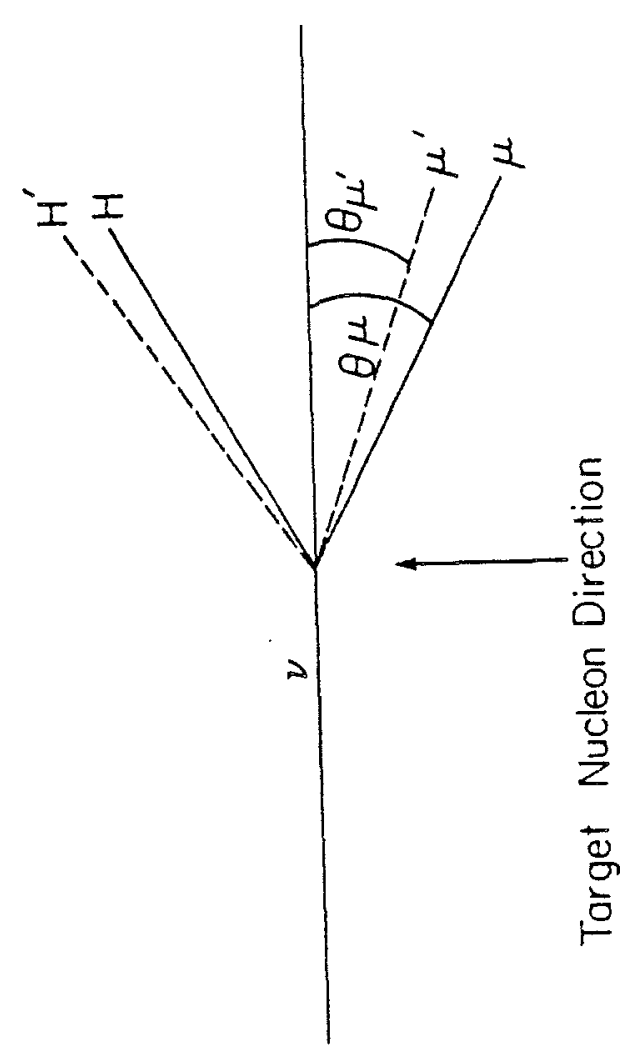



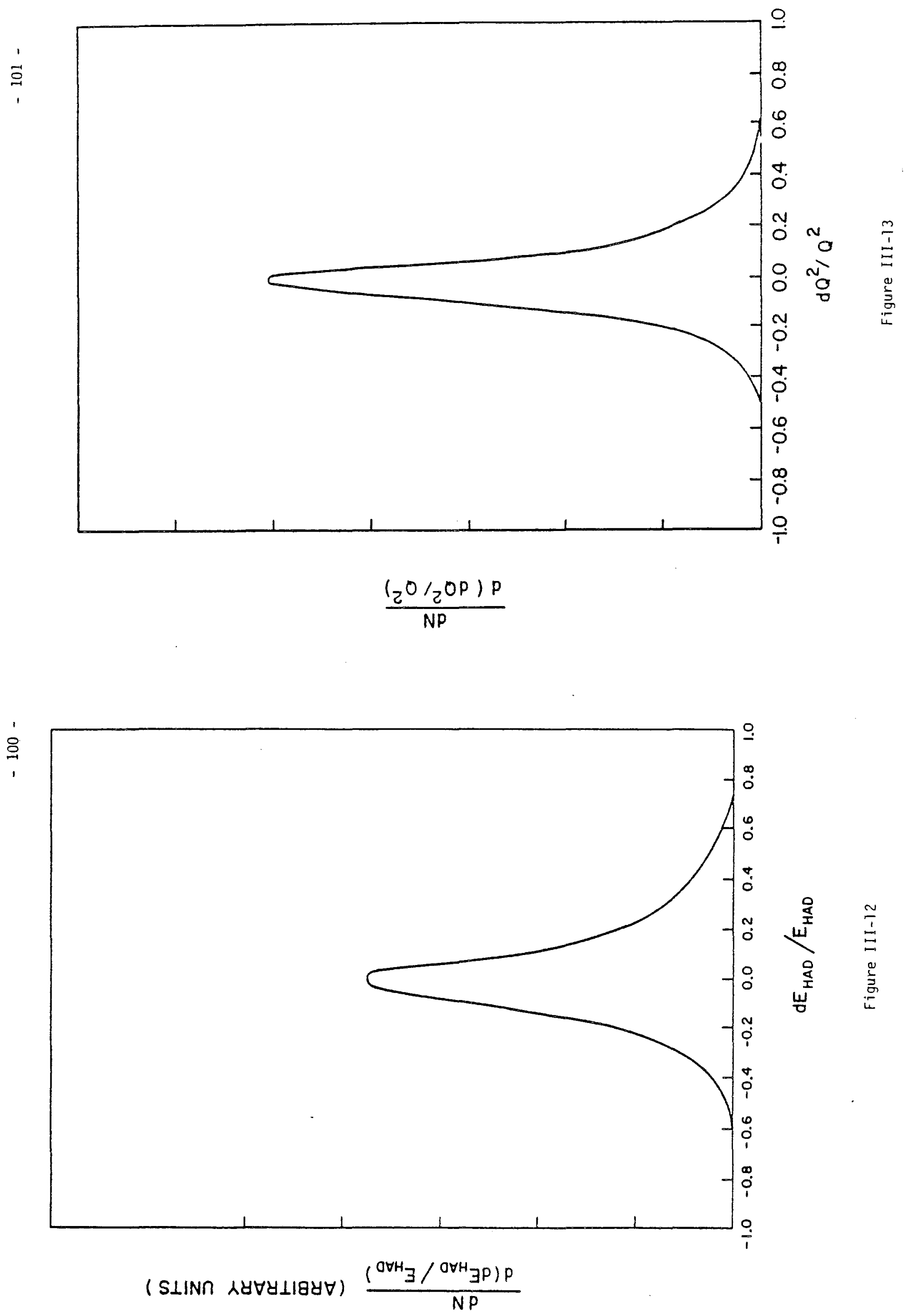

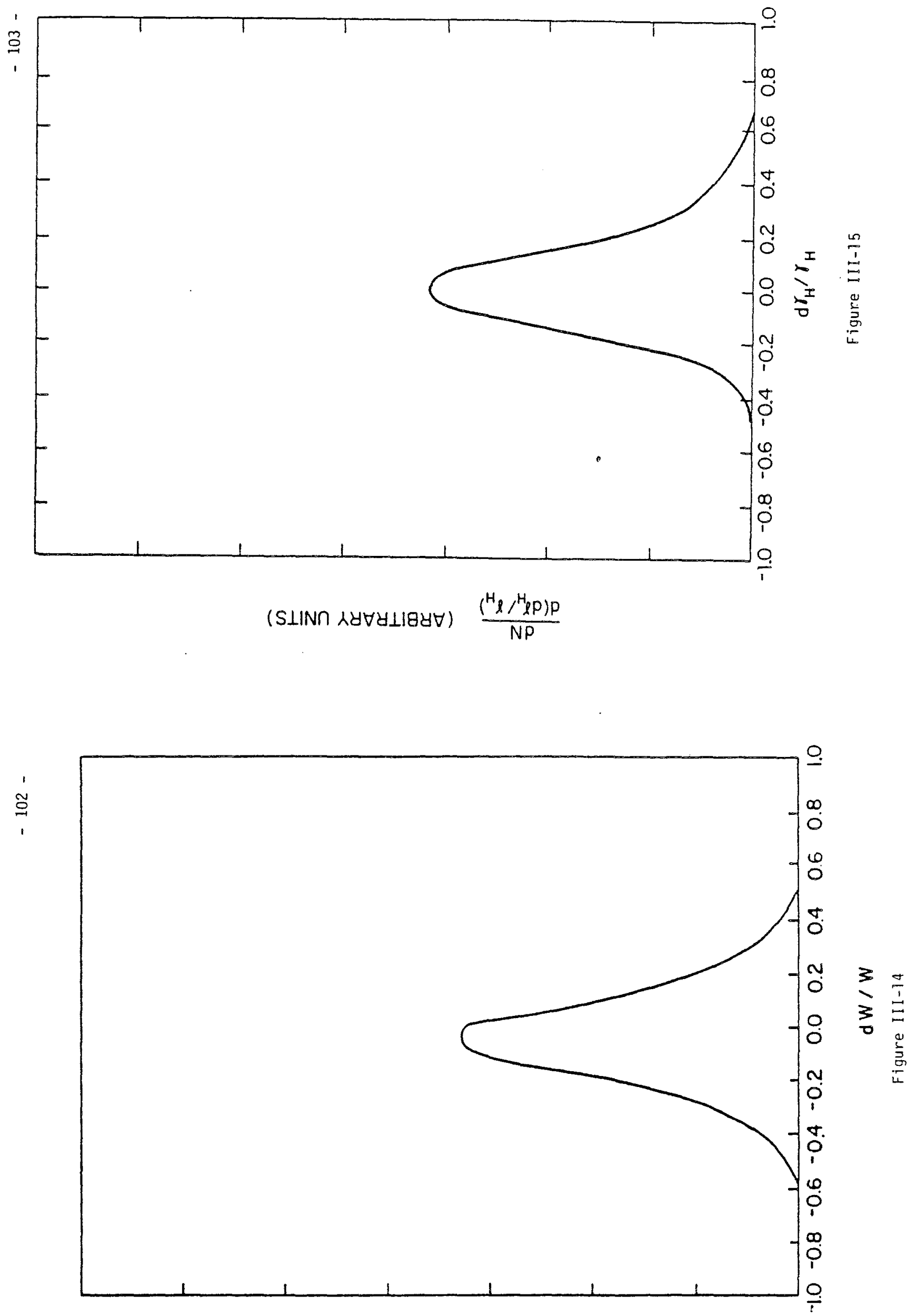

(st!un NsDd!!QدV) $\frac{(M / M P) P}{N P}$ 
1

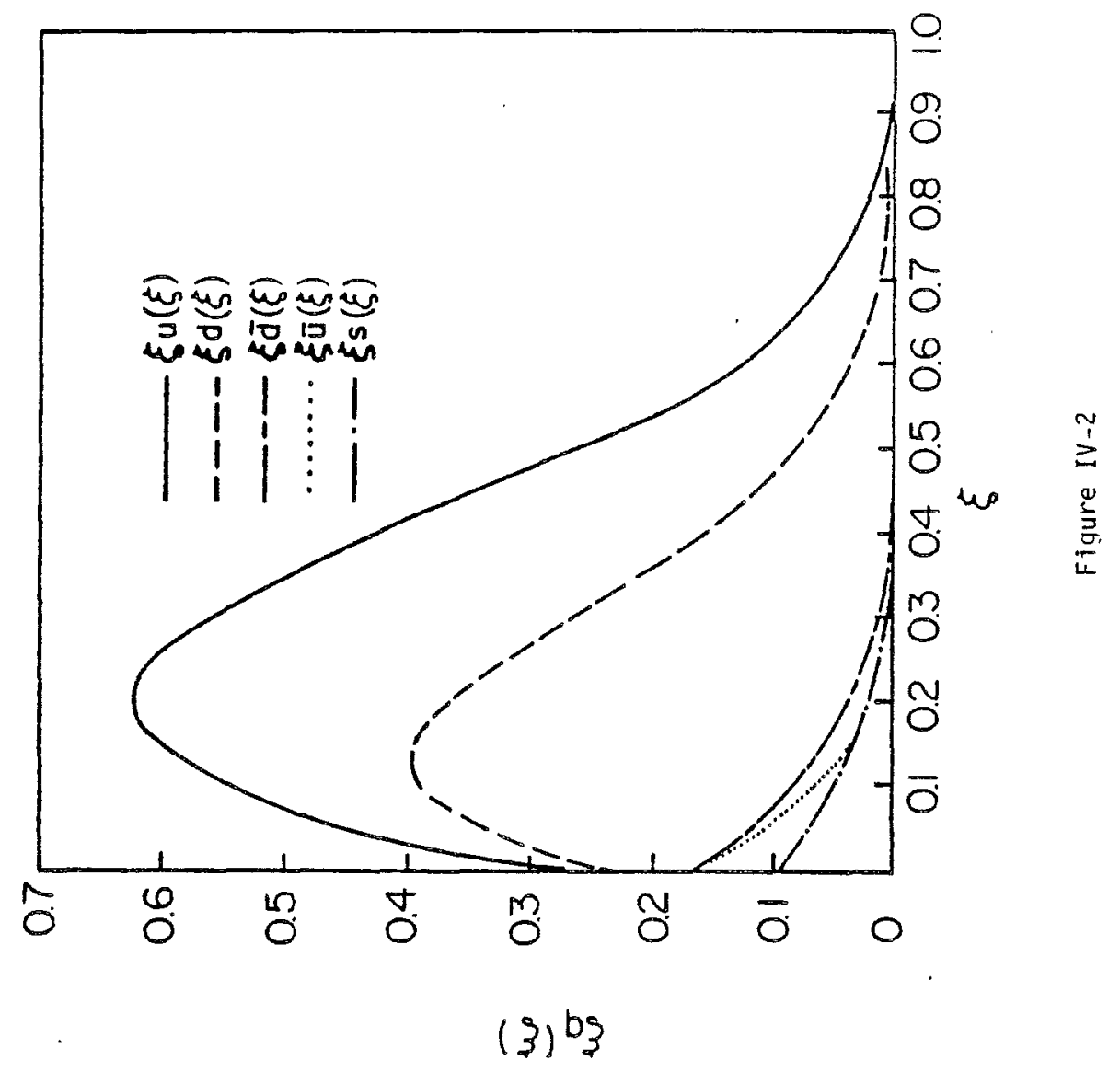

$\stackrel{1}{\circ}$

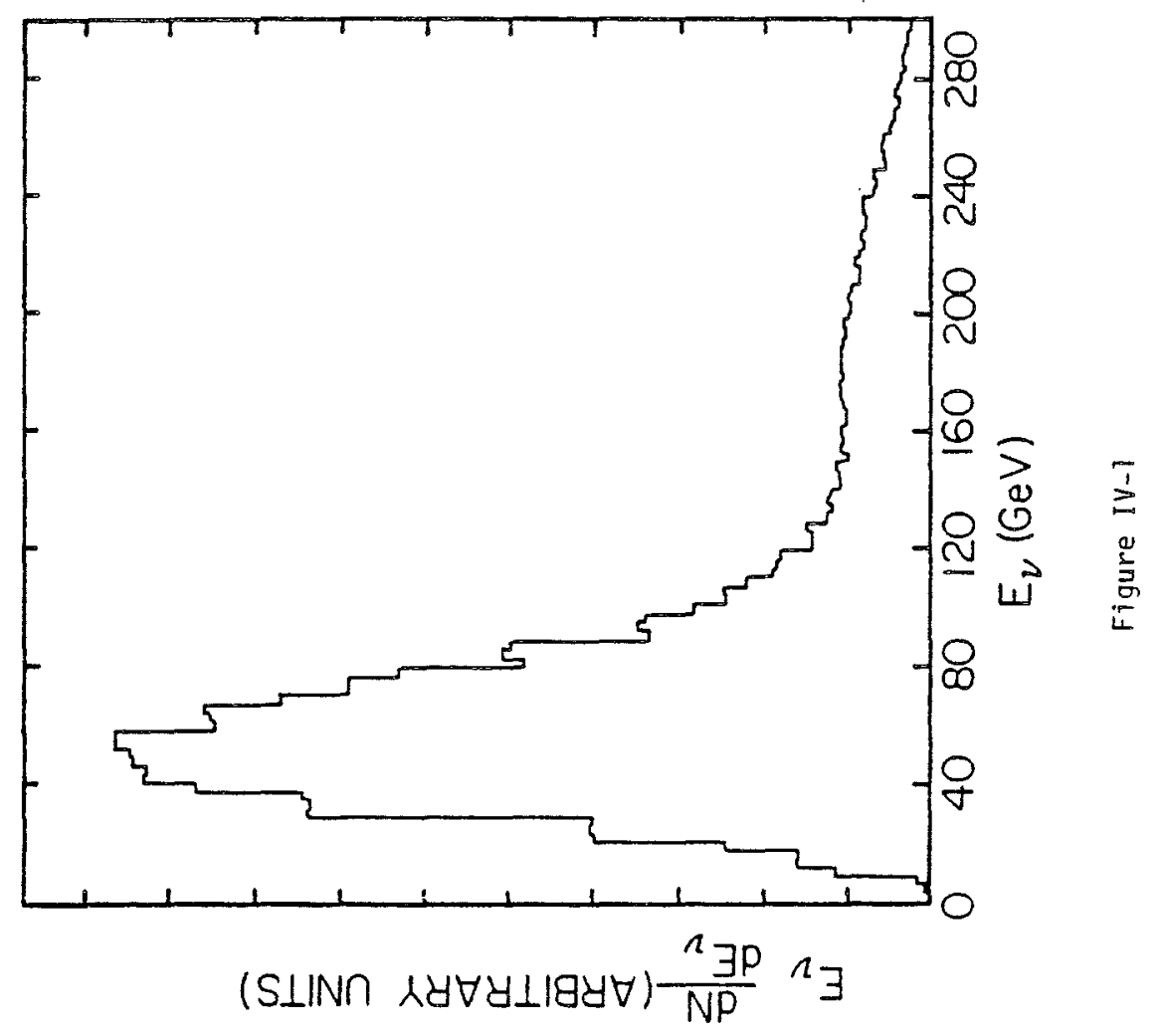




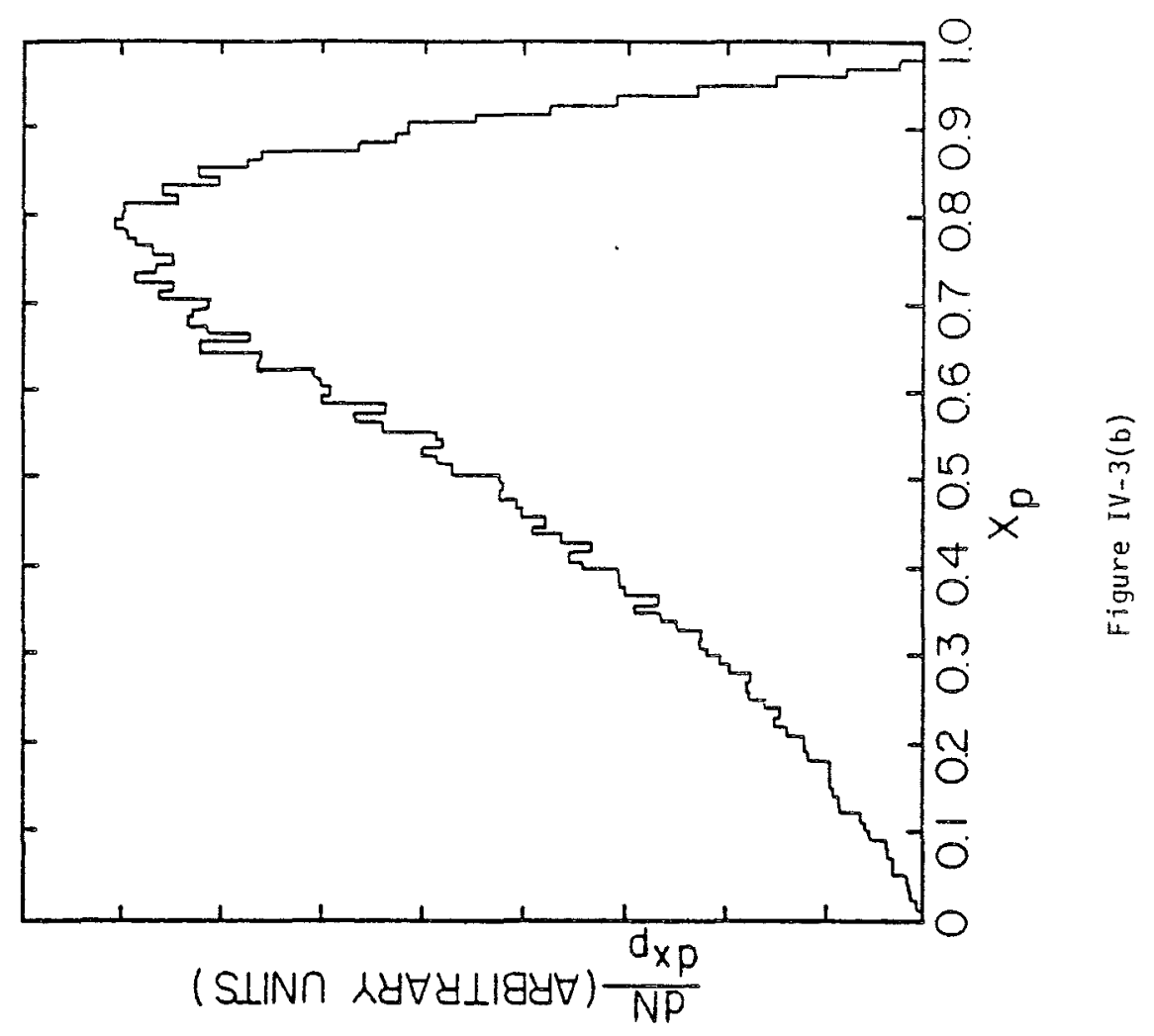

!

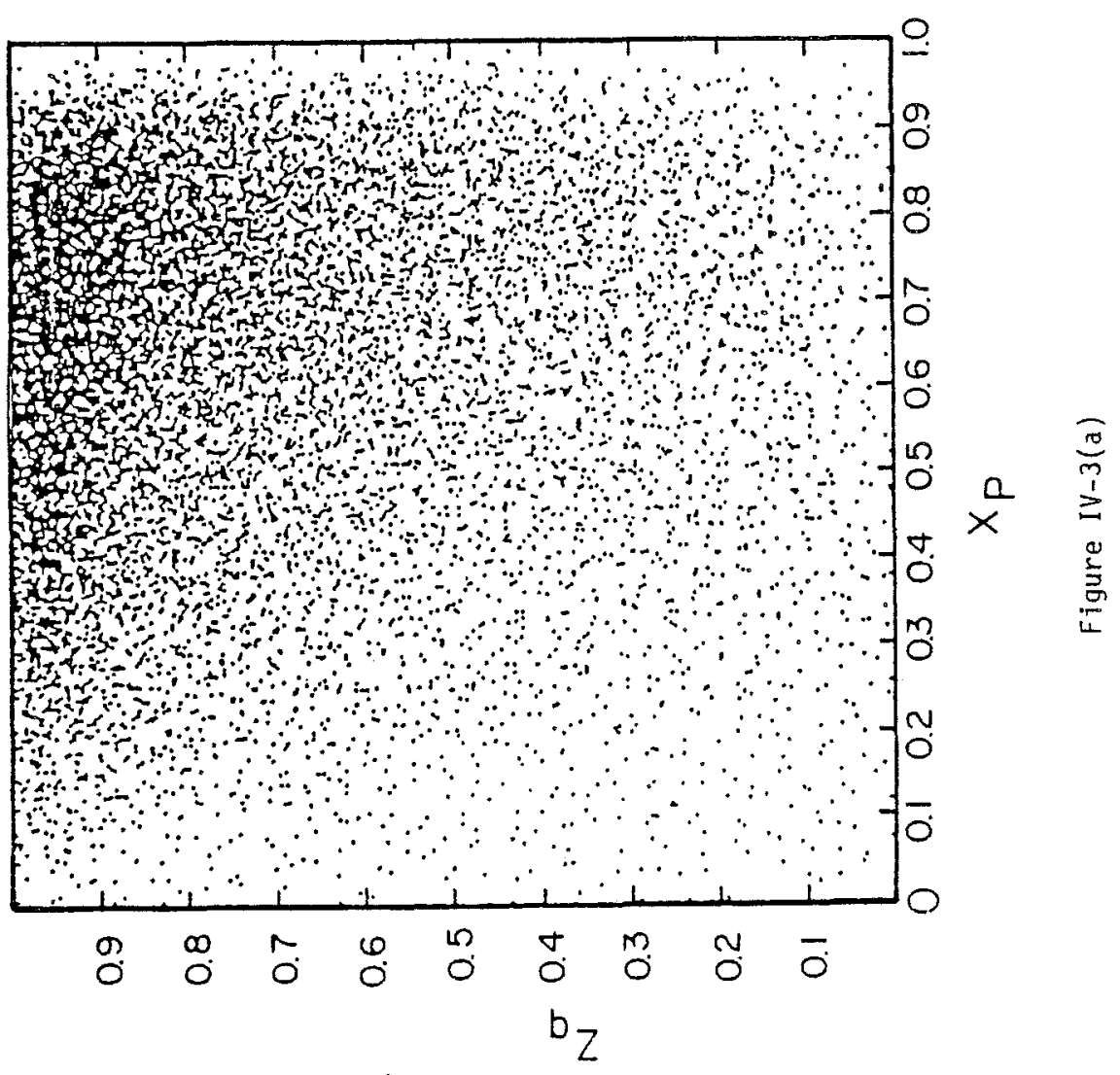




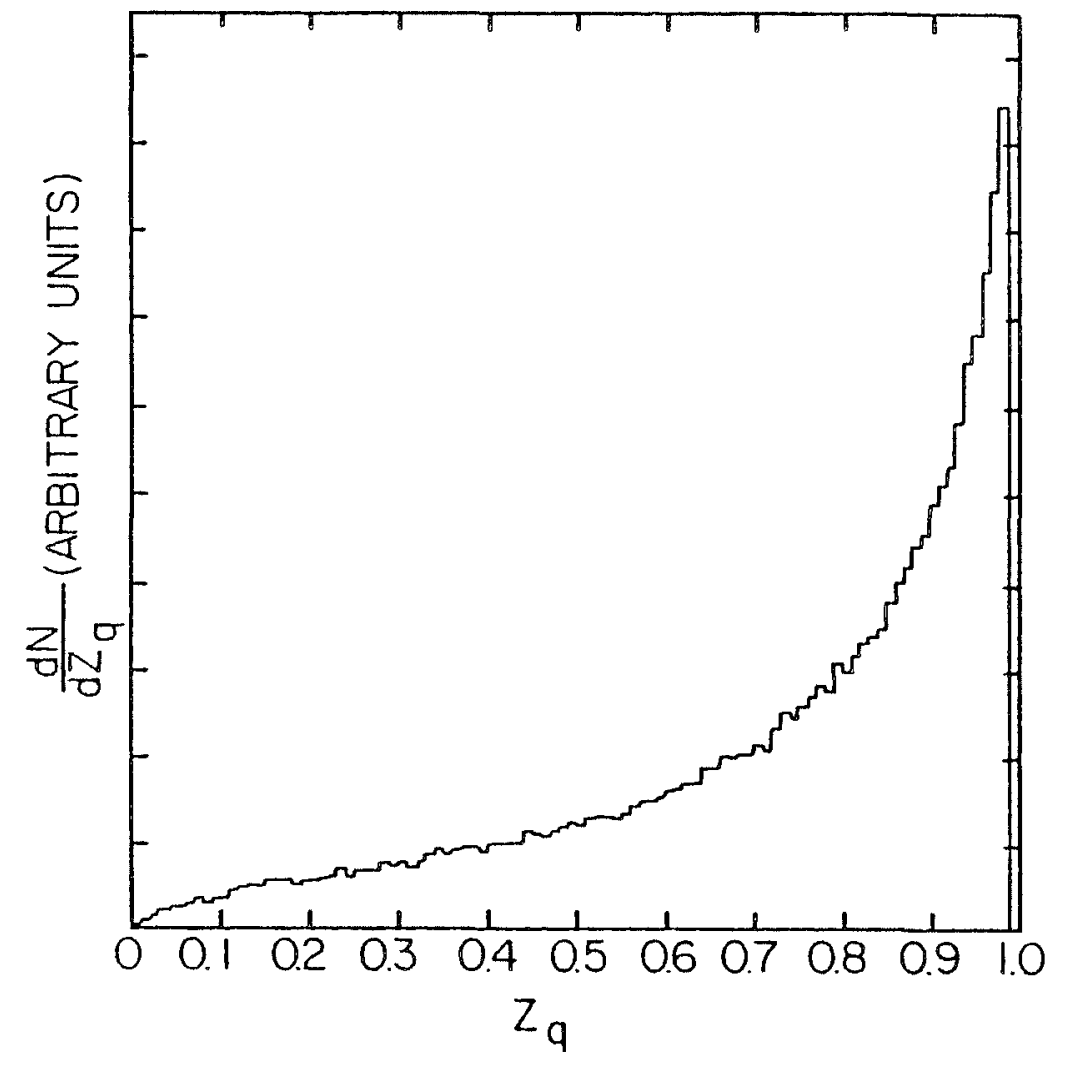

Figure IV-3(c)

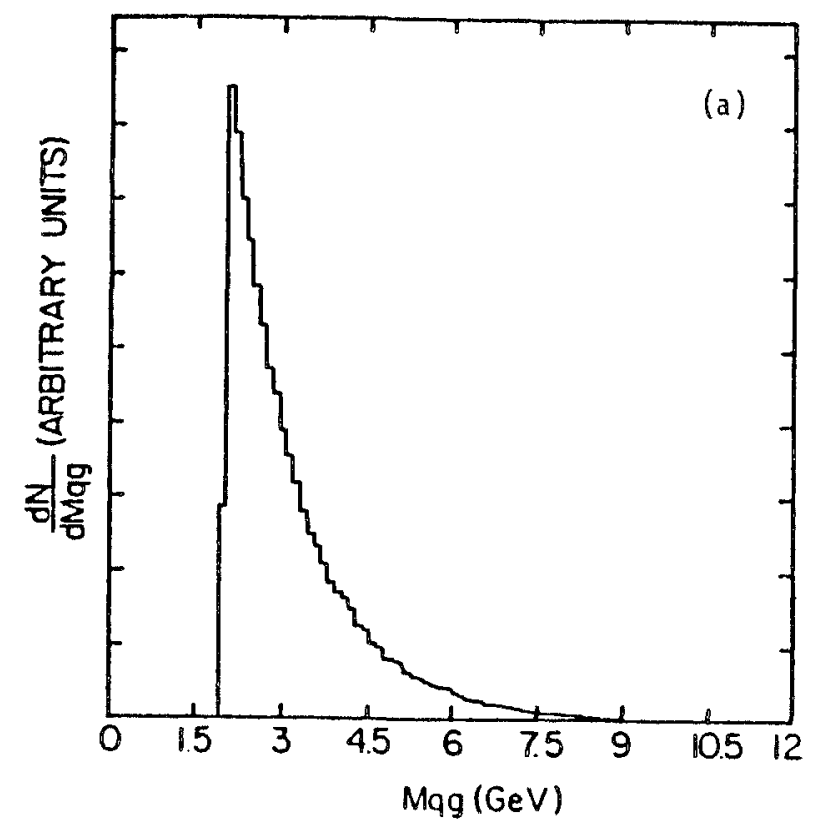

$-109-$

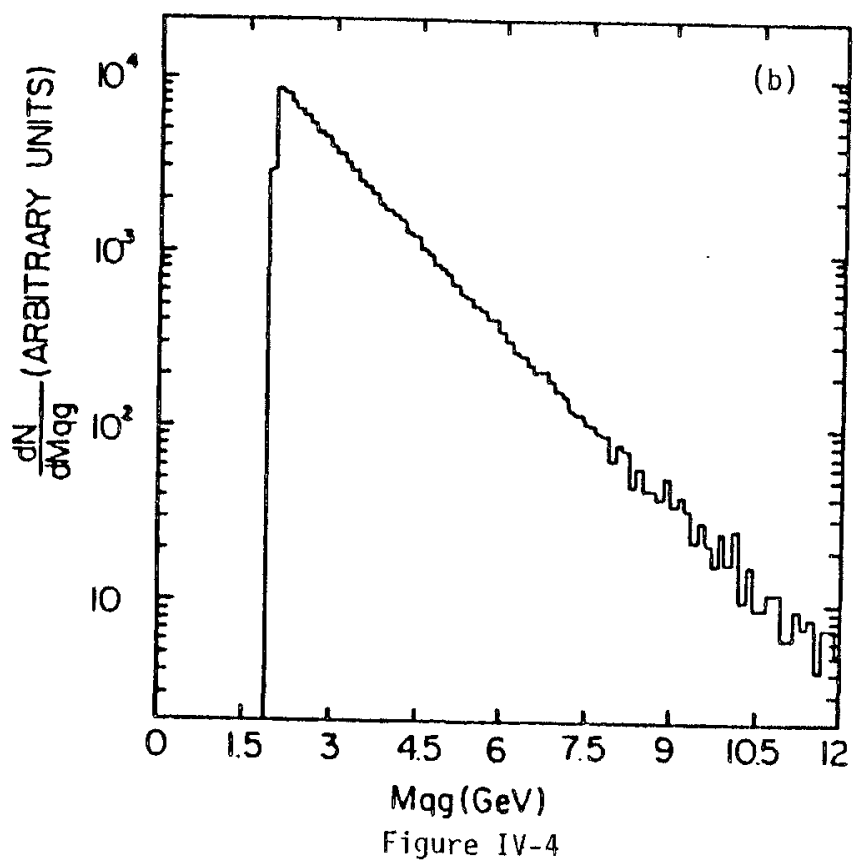




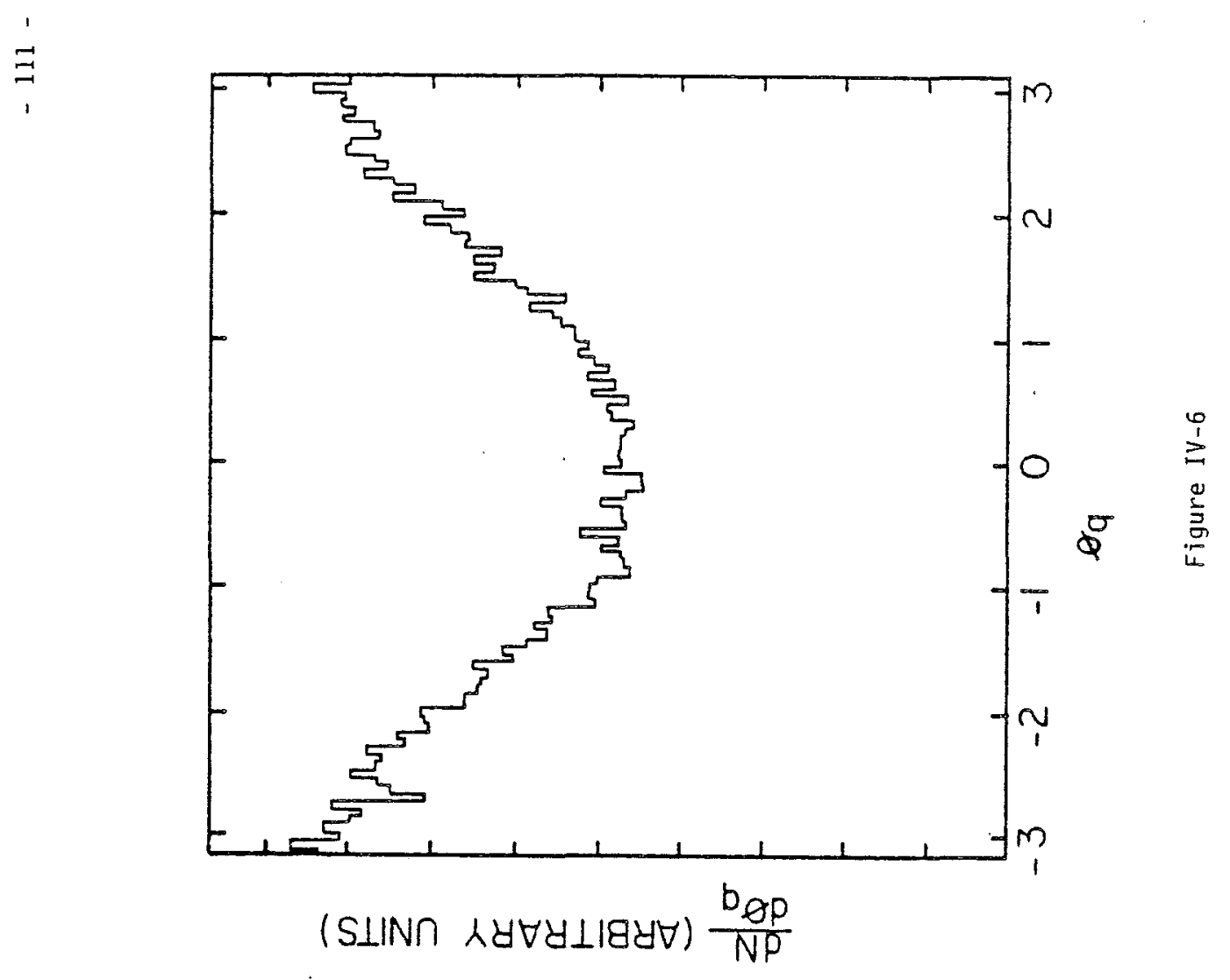

$\stackrel{1}{0}$
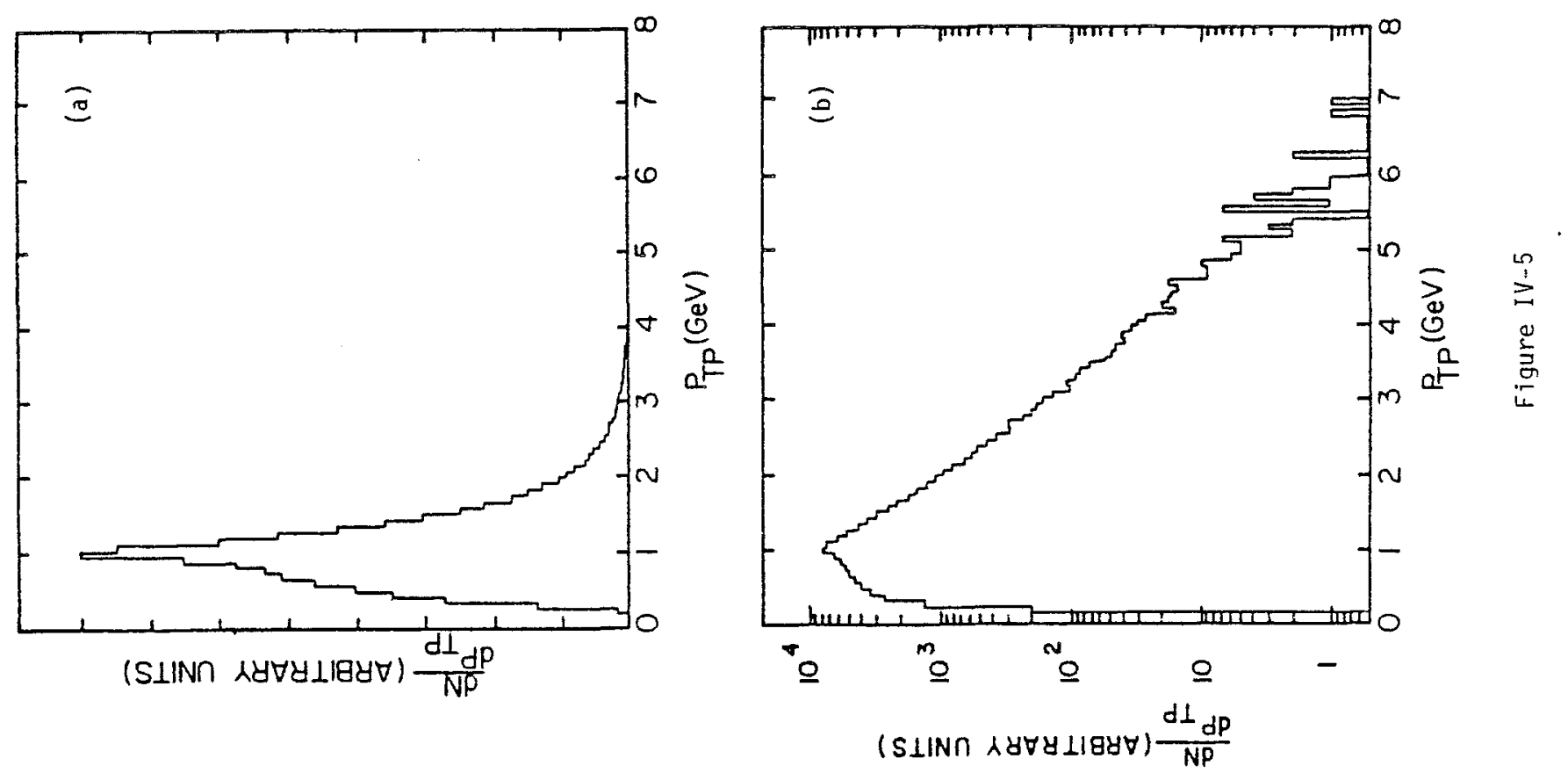
$\stackrel{1}{m}$

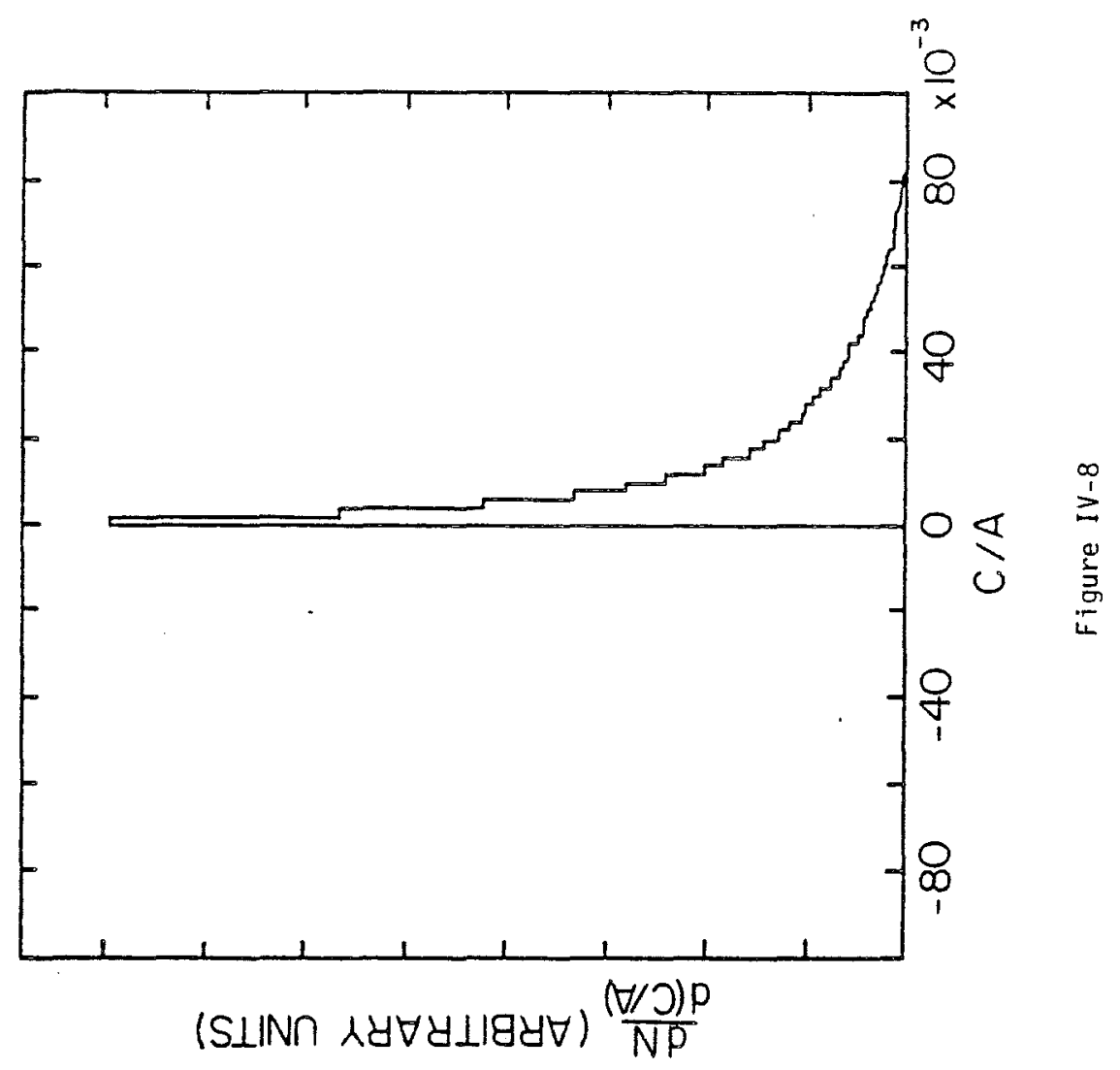

$\stackrel{1}{=}$

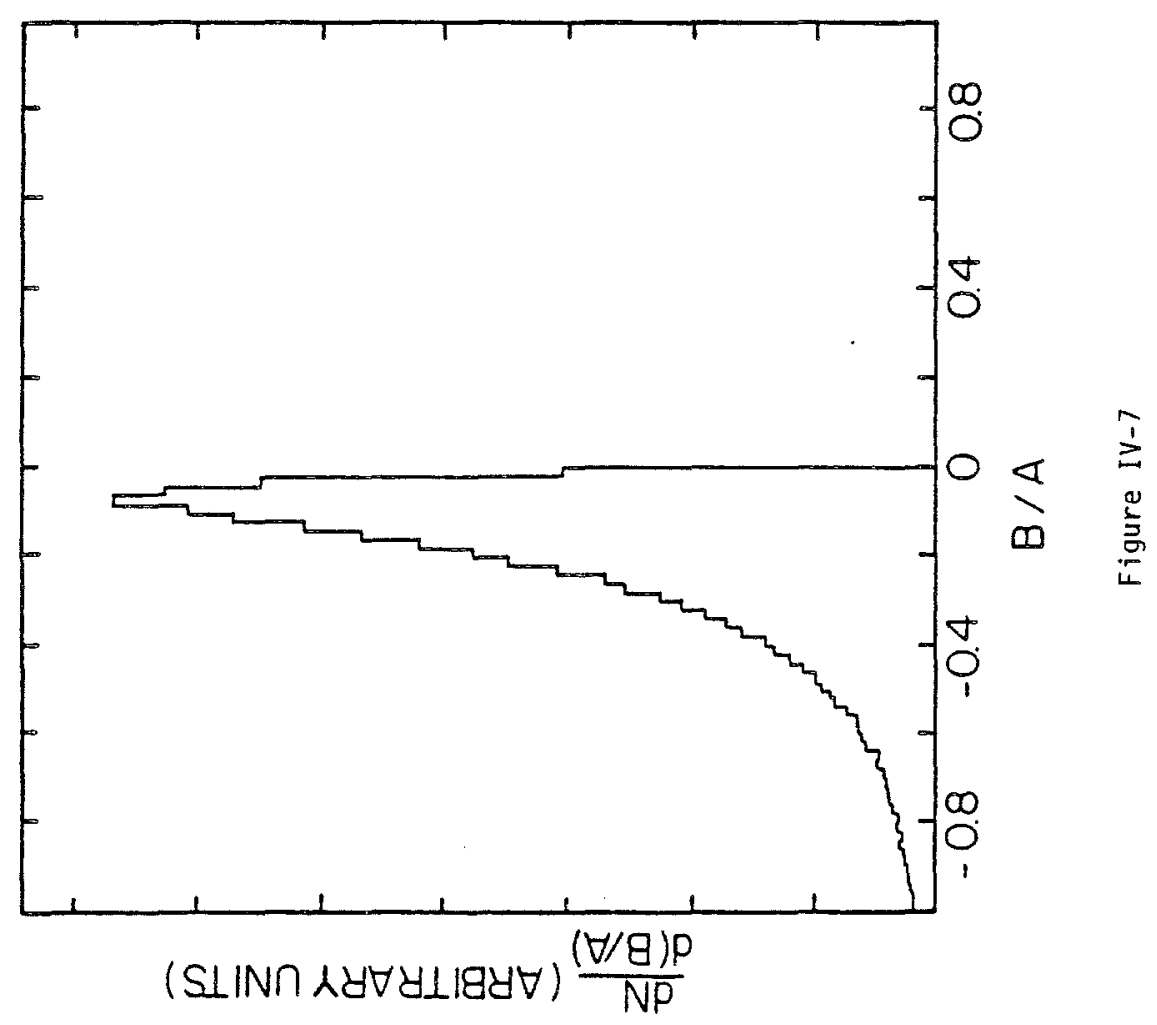


"HIERARCHY" OF FINAL MESONS

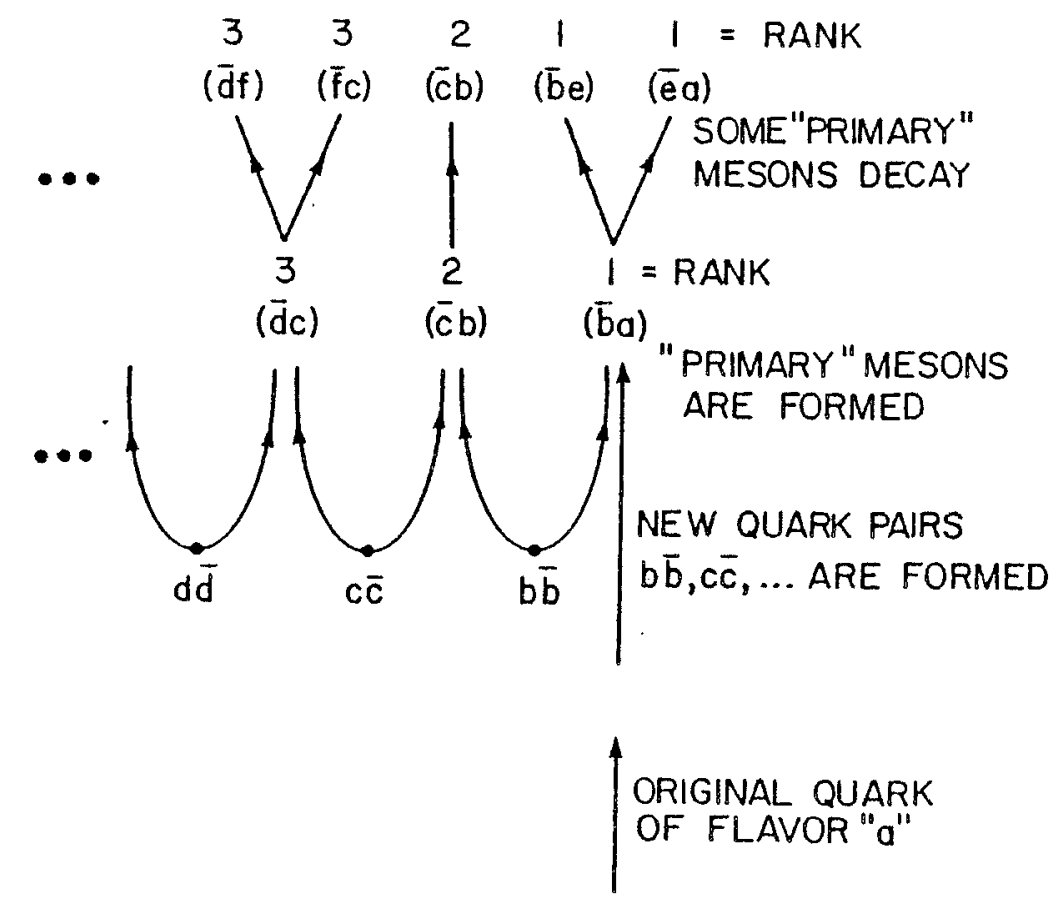

Figure IV-9

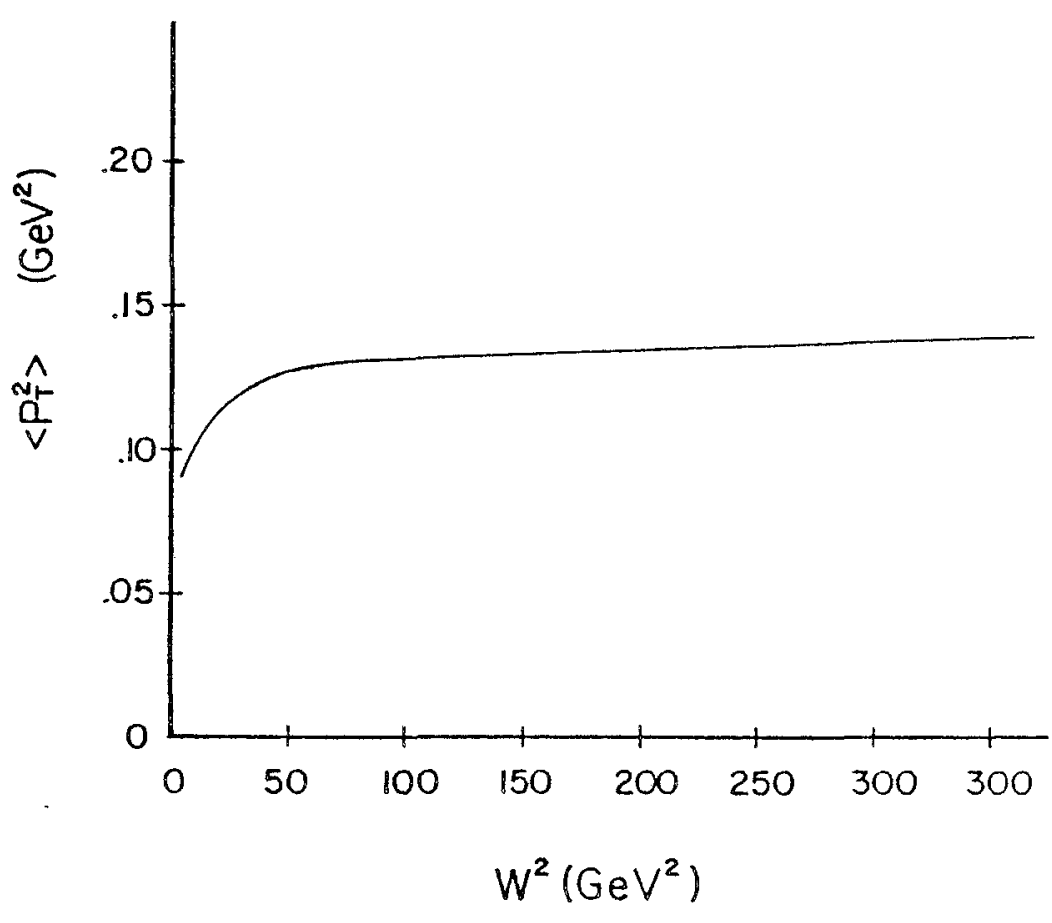

Figure IV-10 


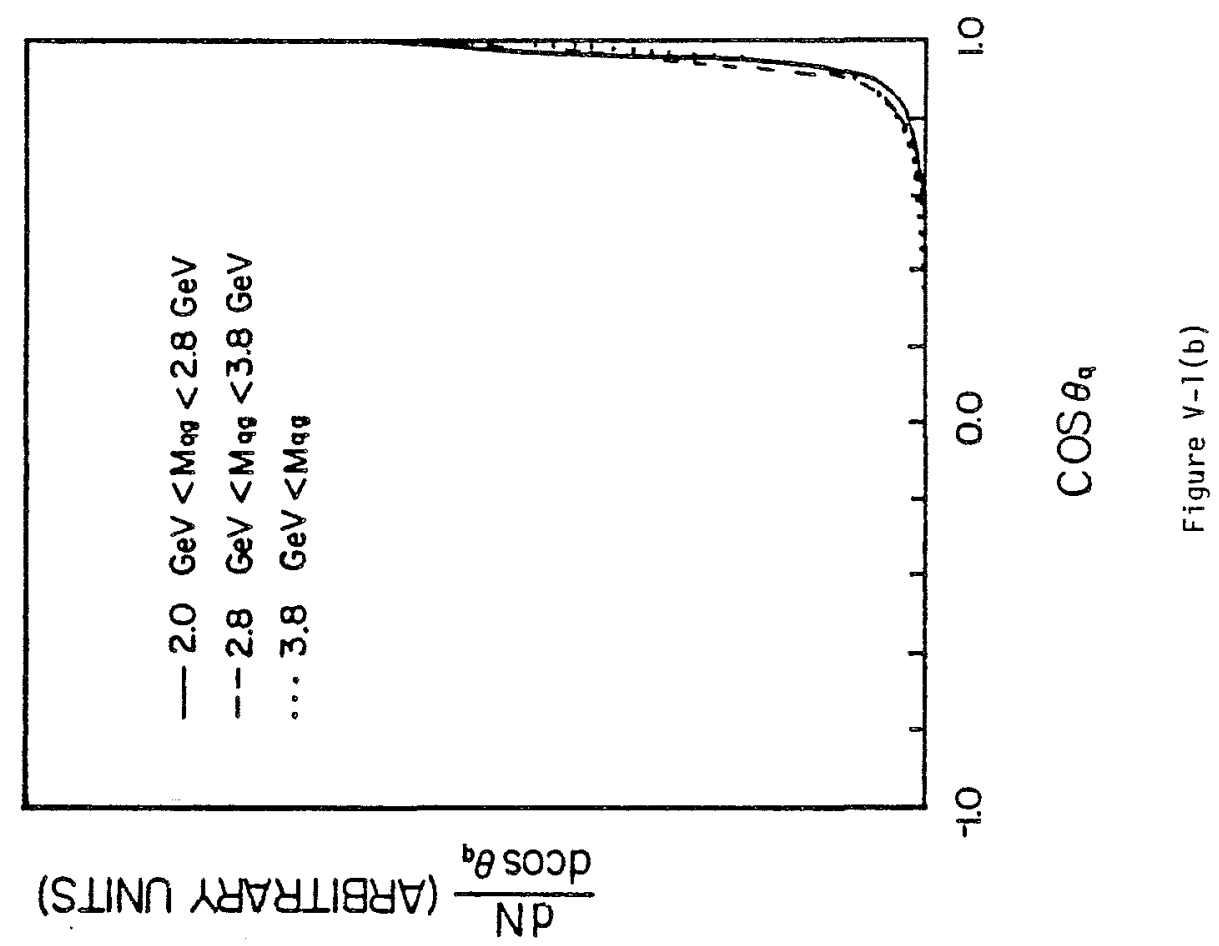

1

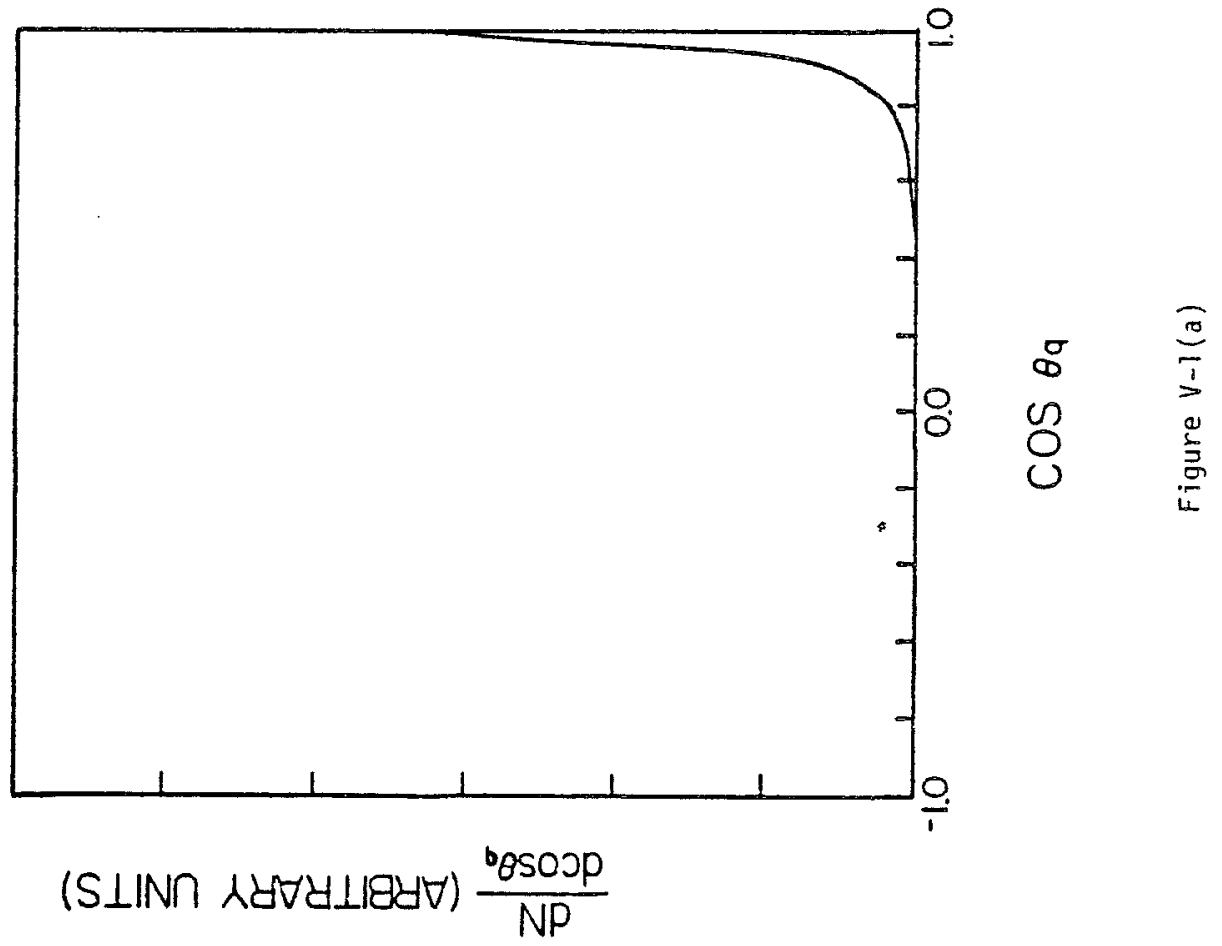




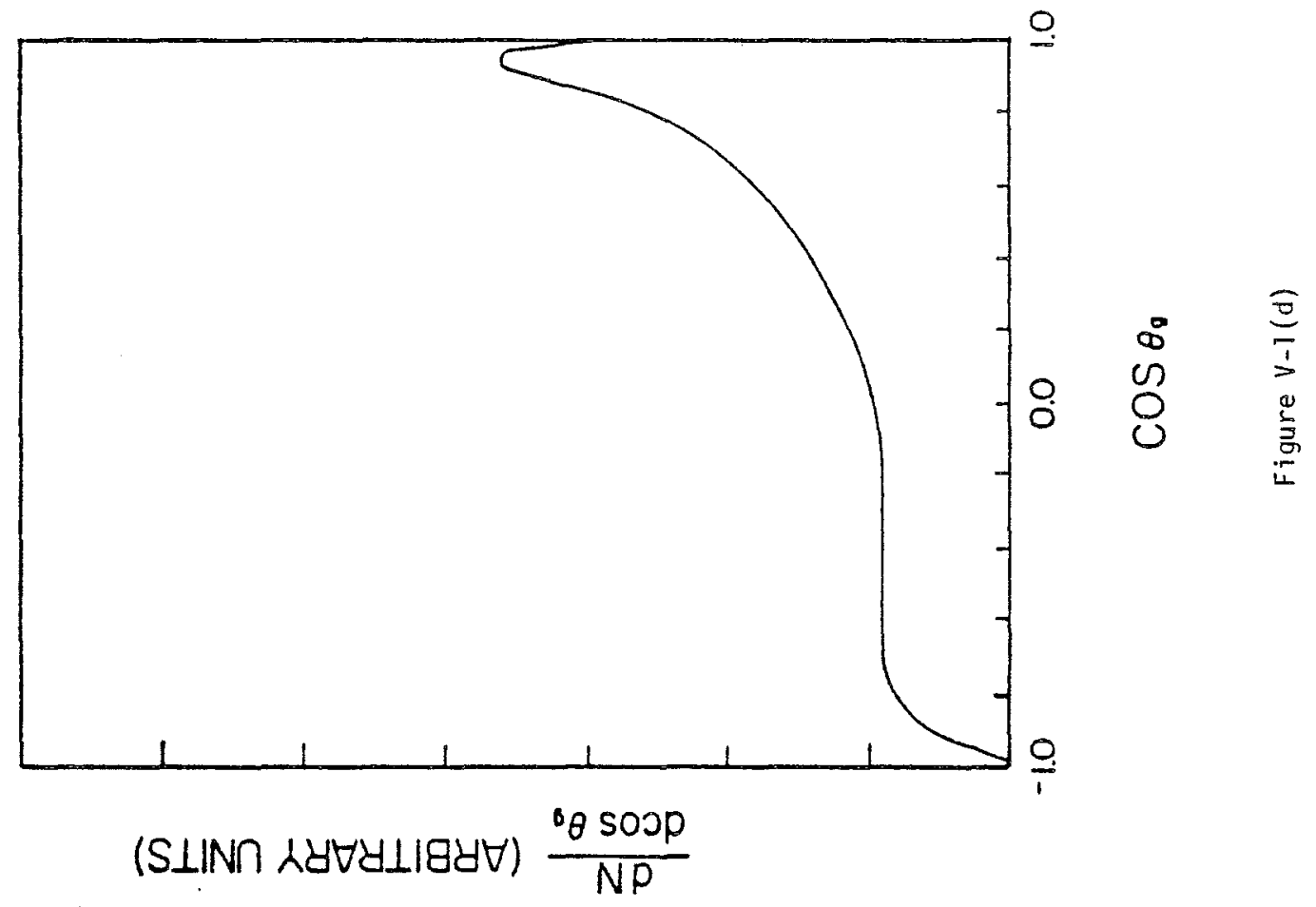

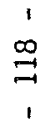

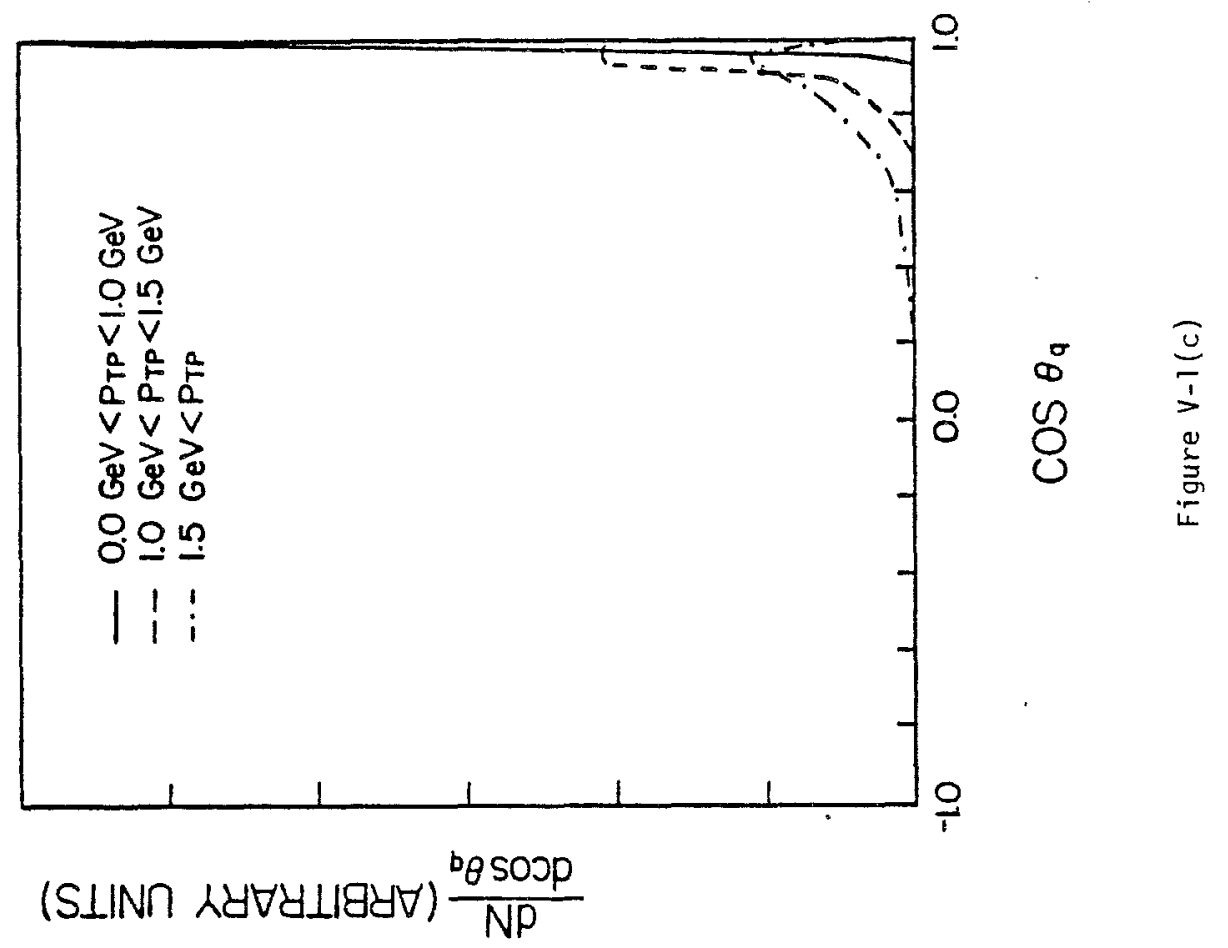



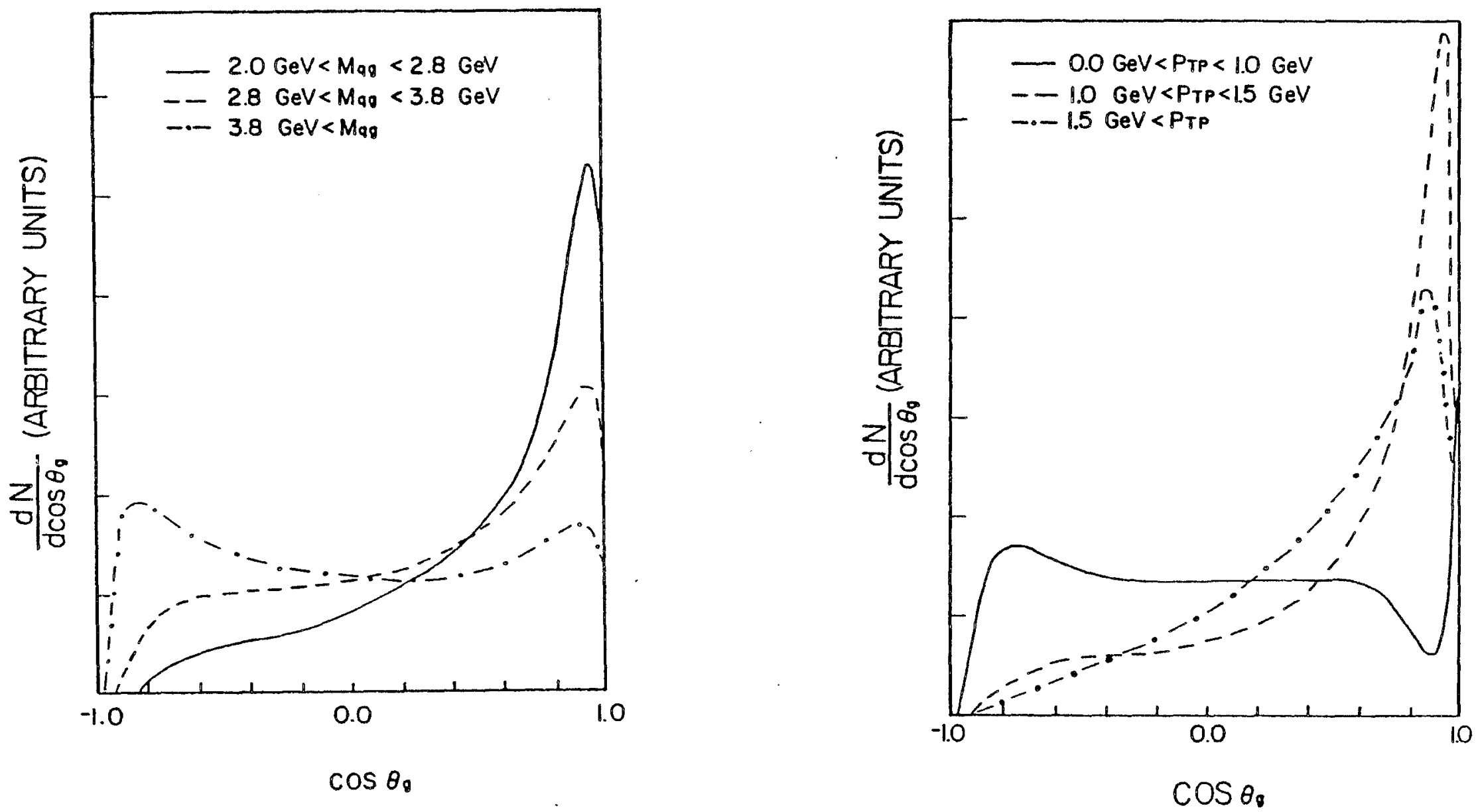

Figure $V-1(e)$

Figure $V-l(f)$ 


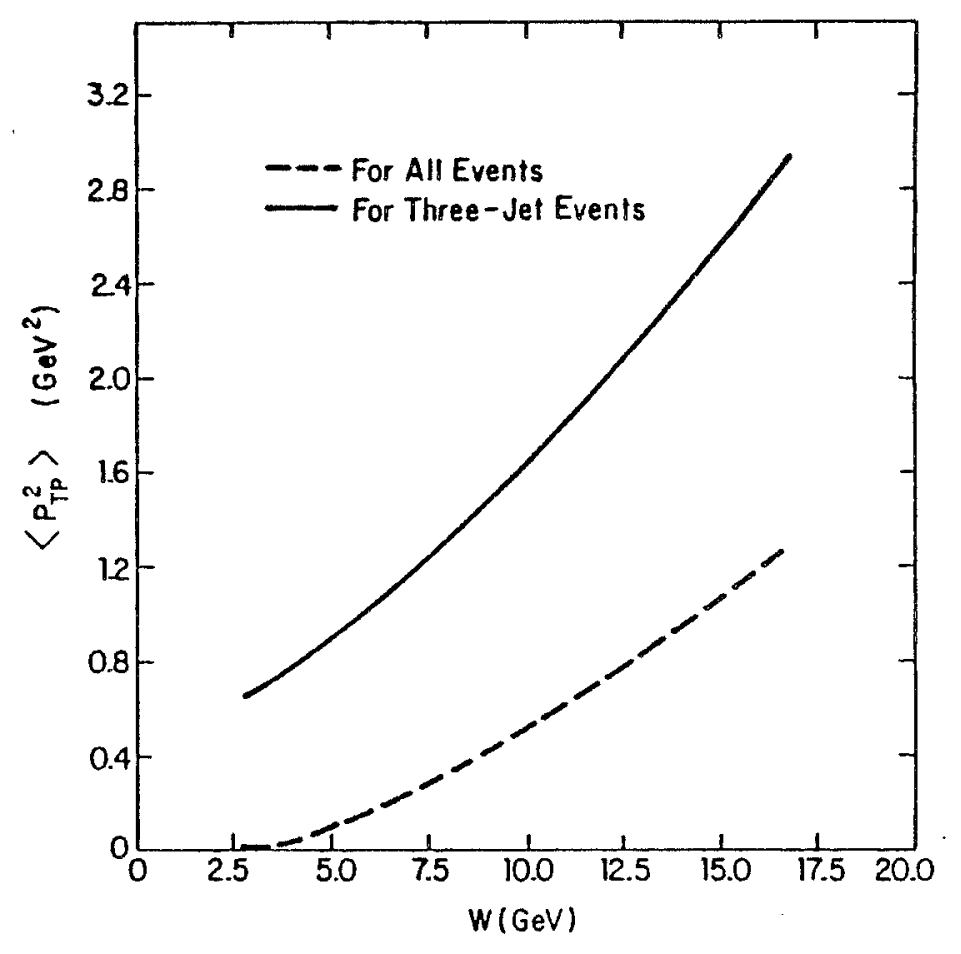

Figure V-2

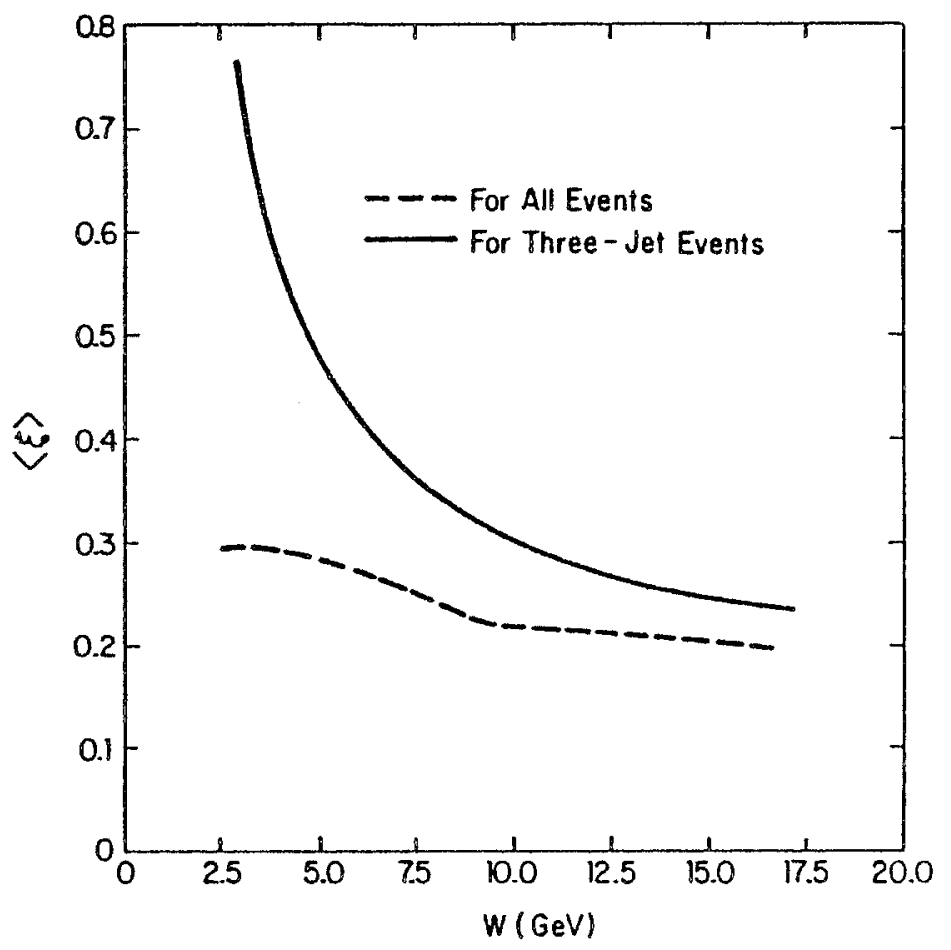

Figure $V-3$ 


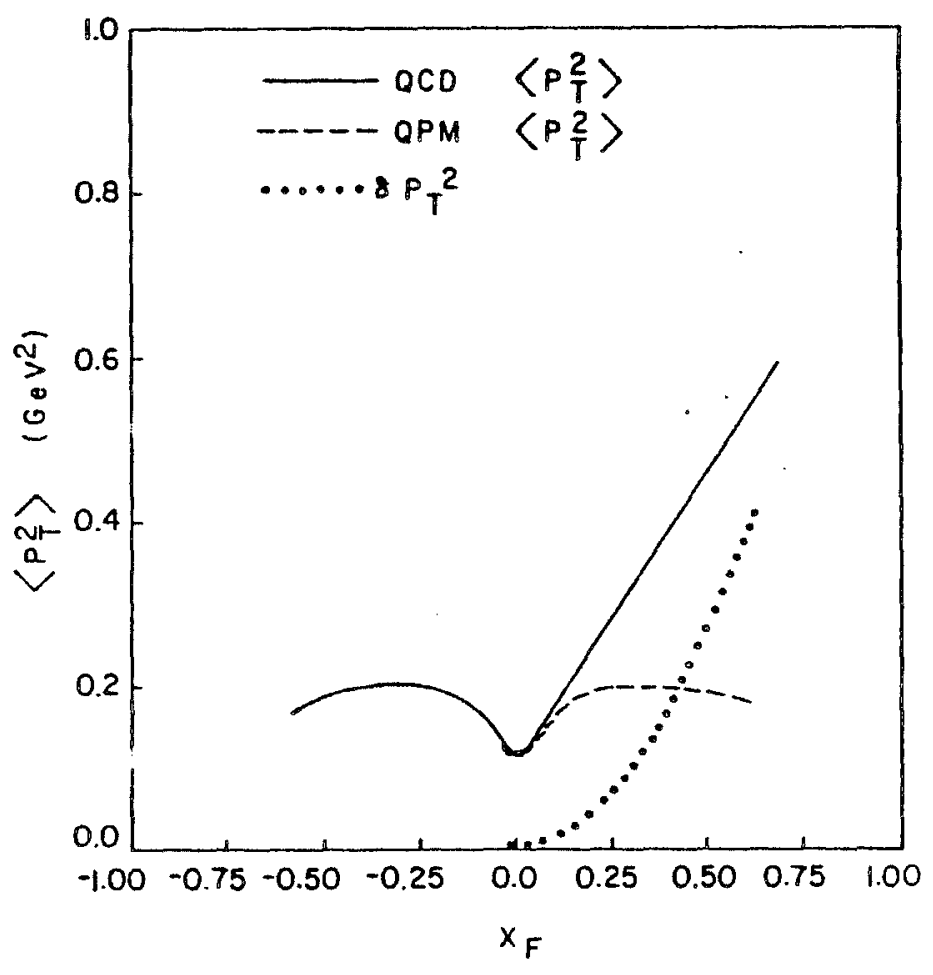

Figure V-4

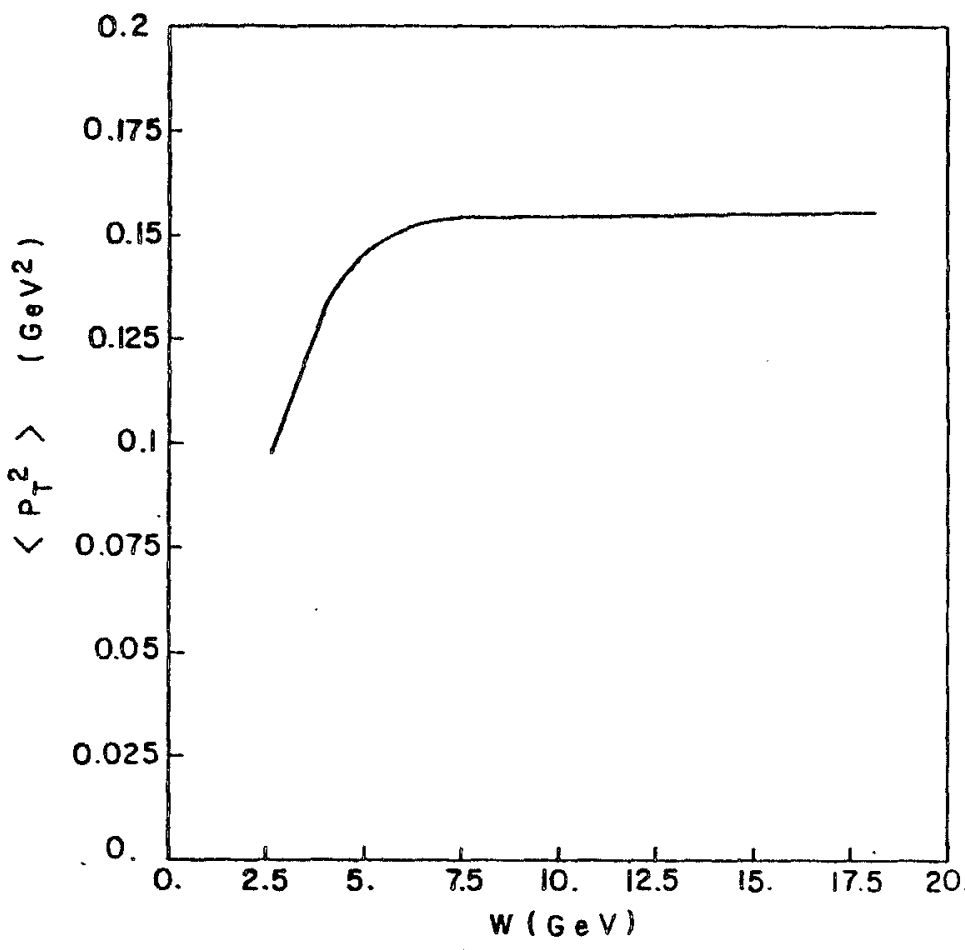

Figure V-5 


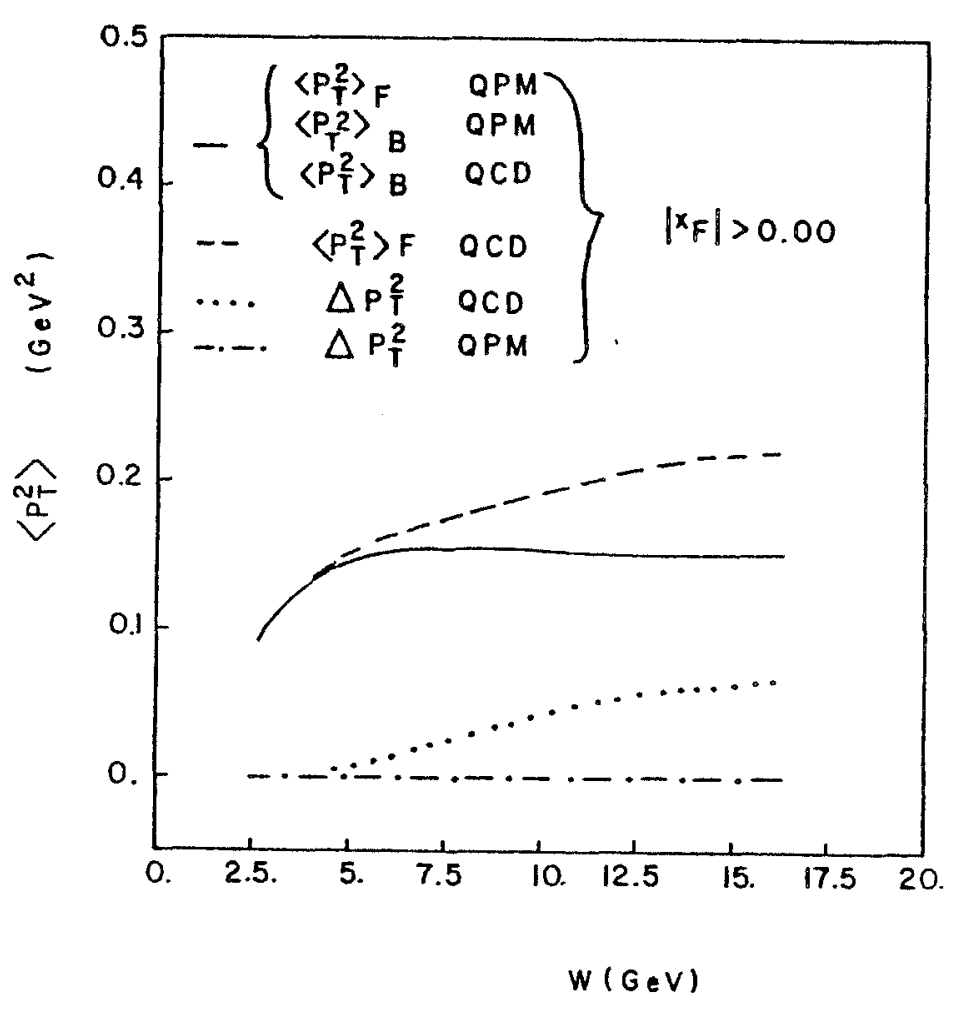

Figure $V-6(a)$

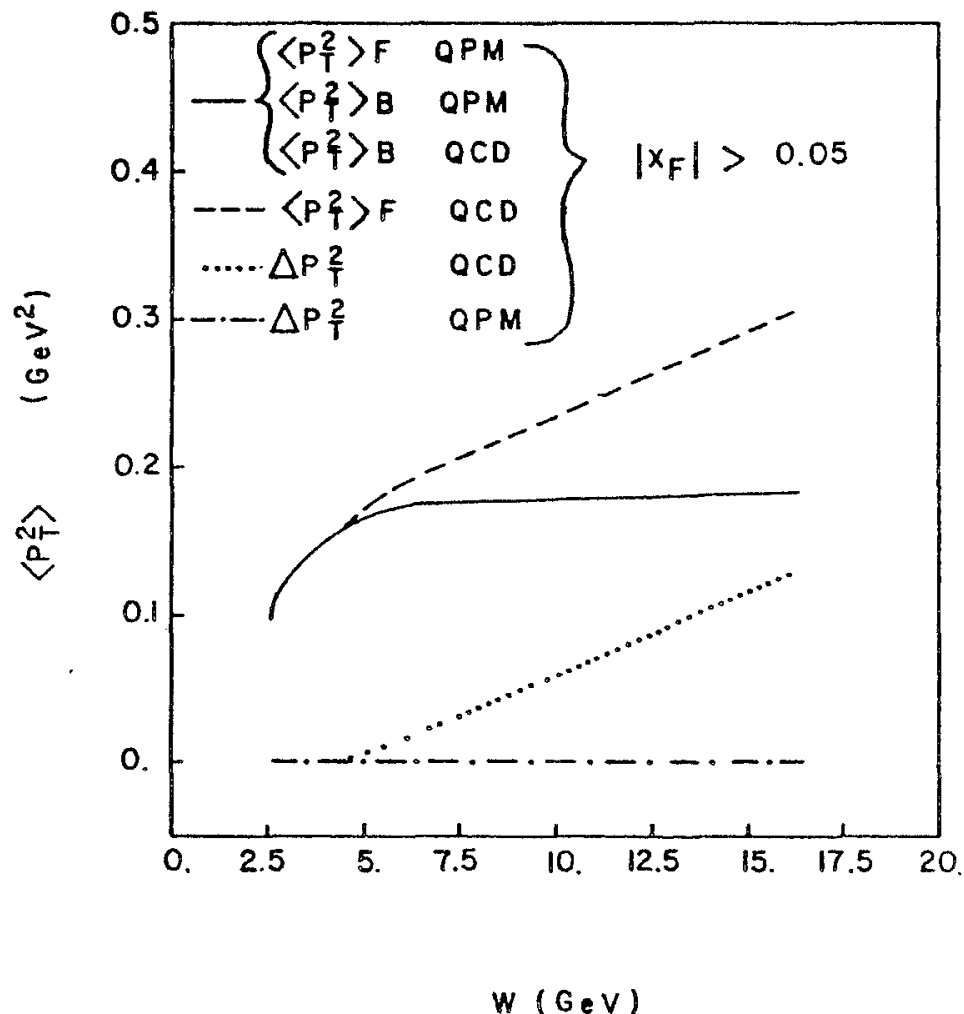

Figure $V-6(b)$ 


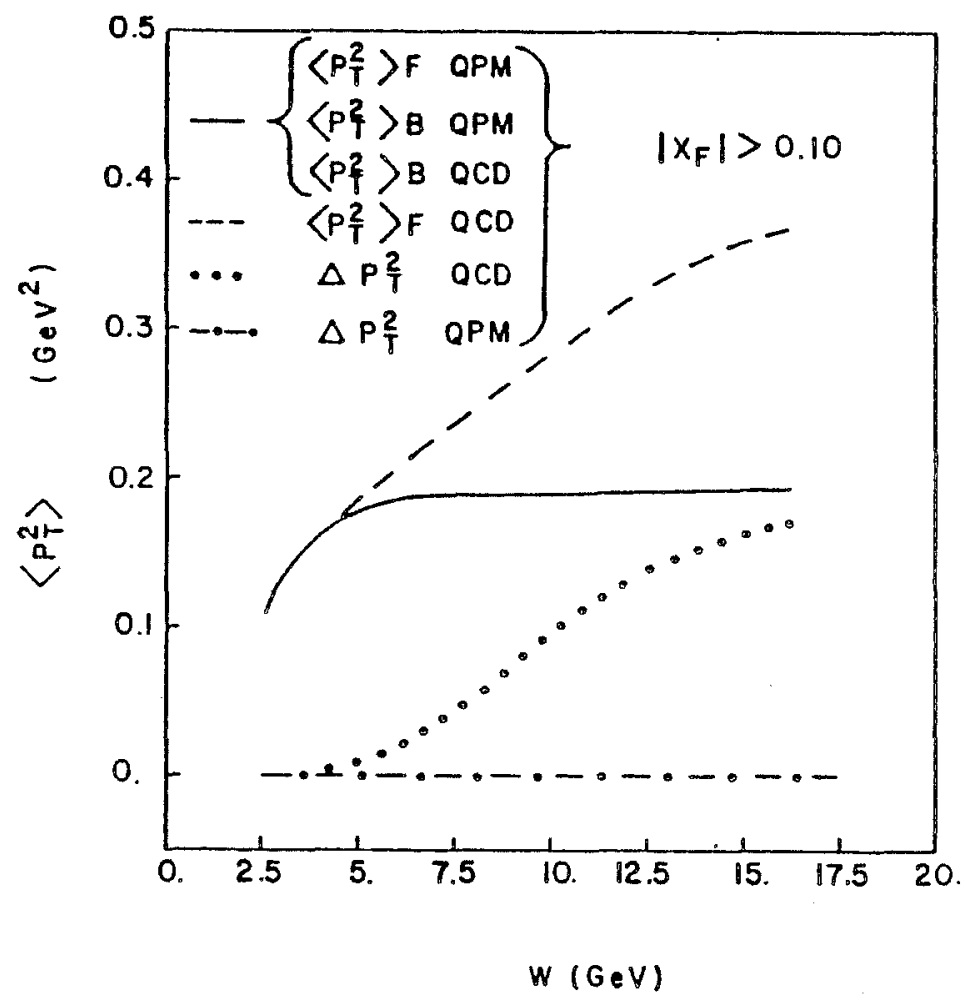

Figure $V-6(c)$

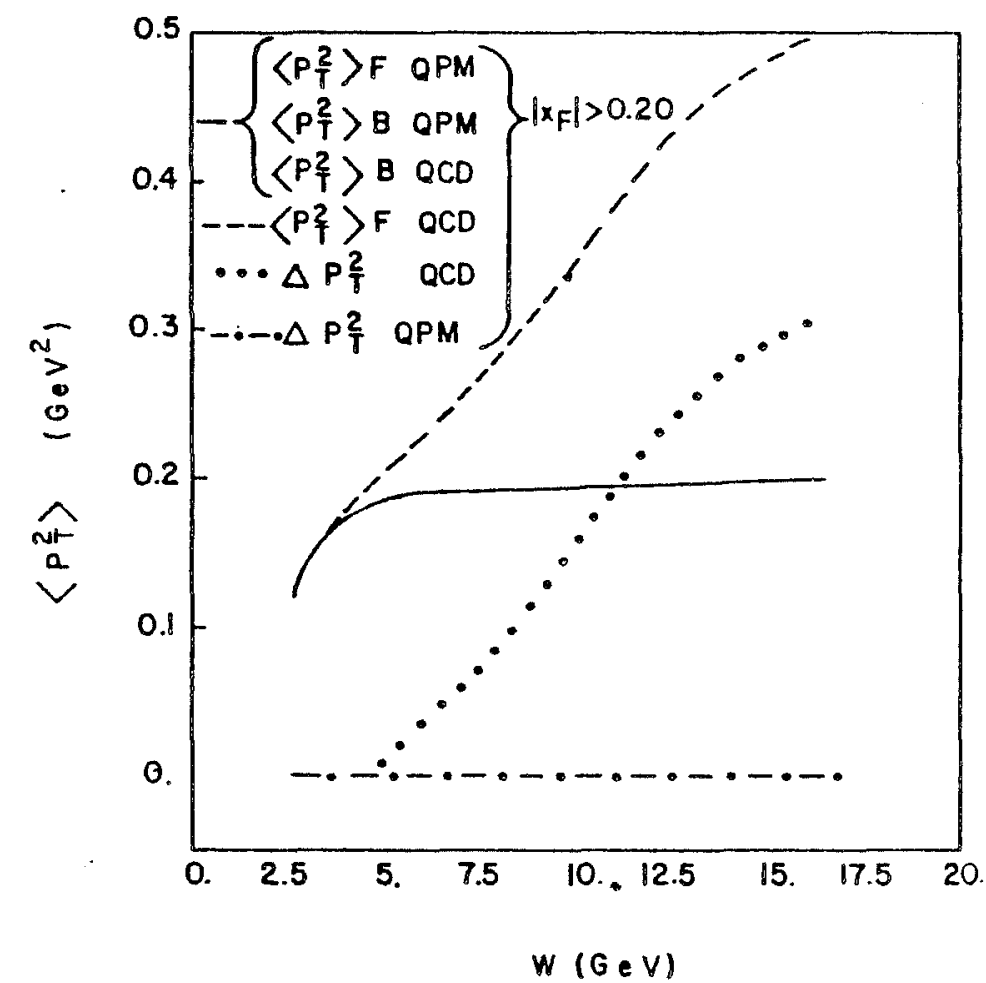

Figure $v-6(d)$ 


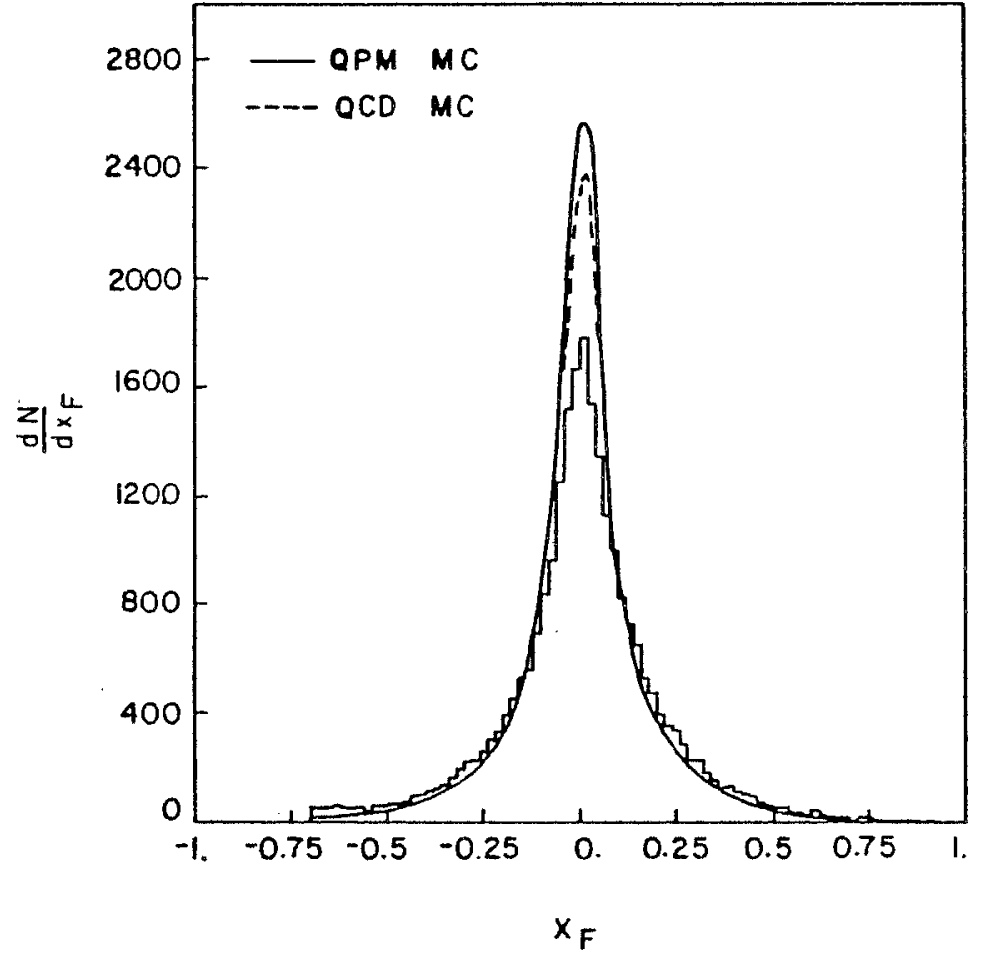

Figure $V-7$

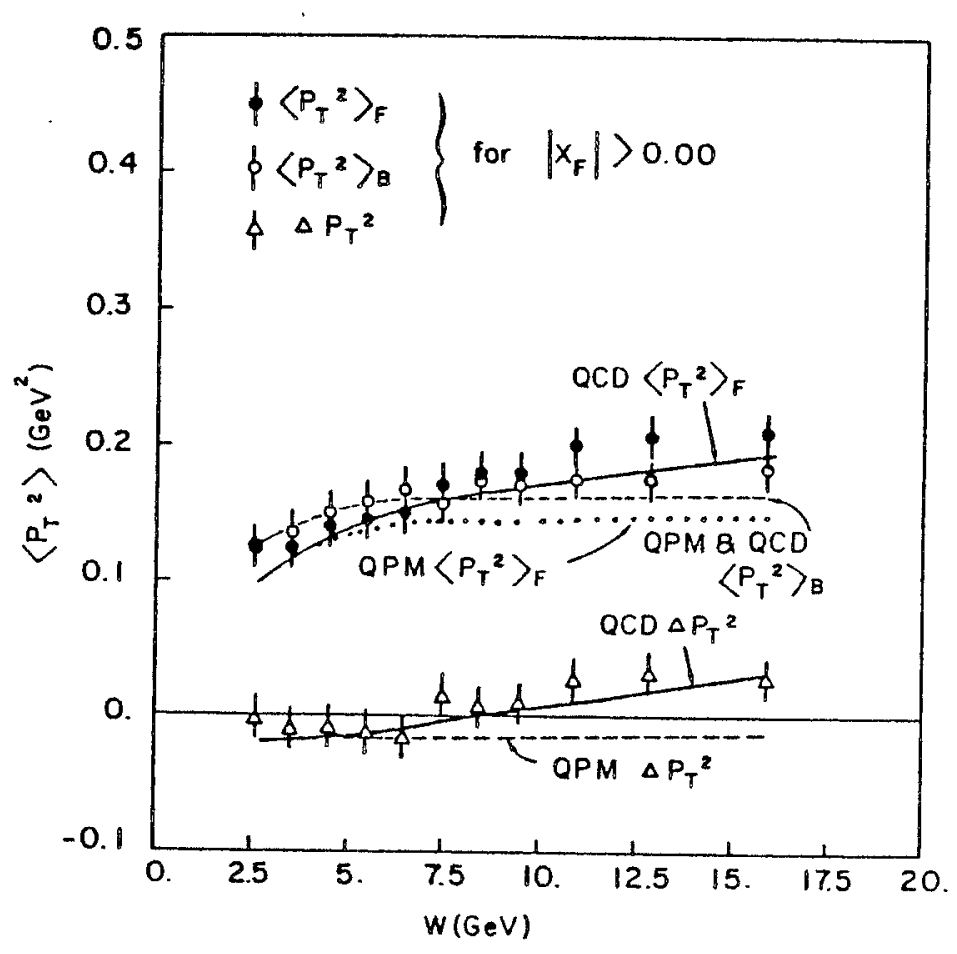

Figure $V-8(a)$ 


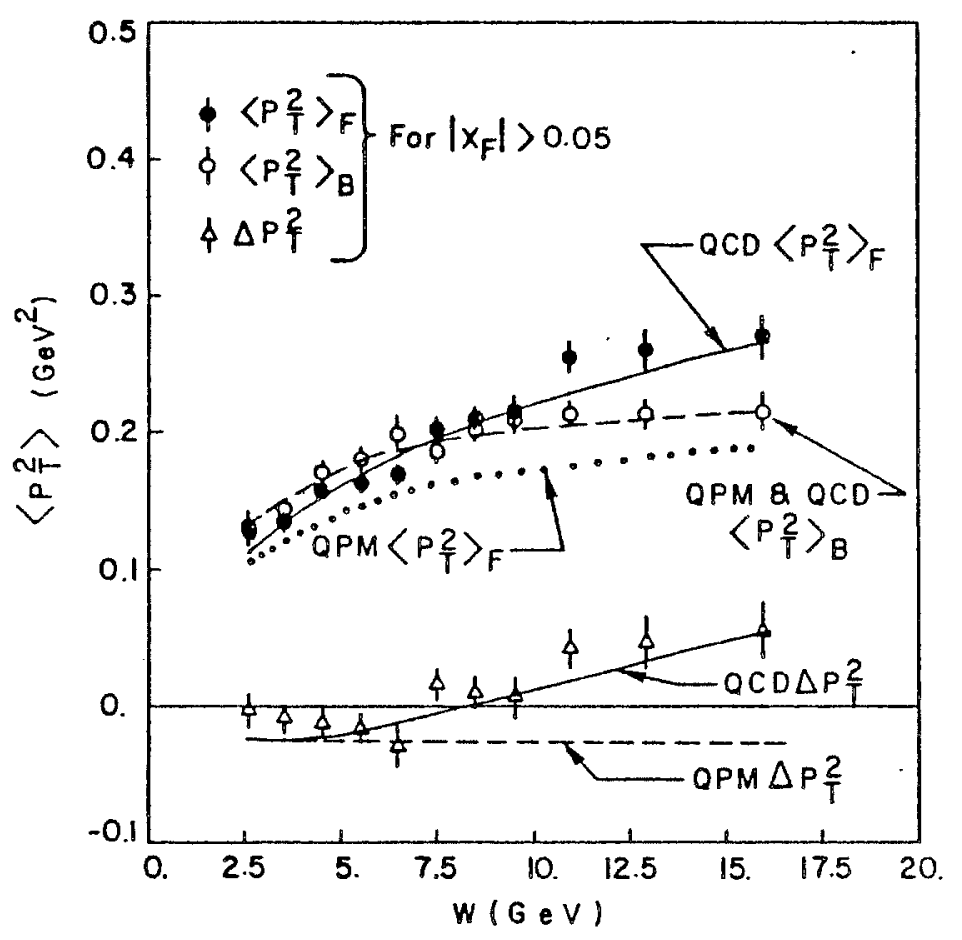

Figure V-8(b)

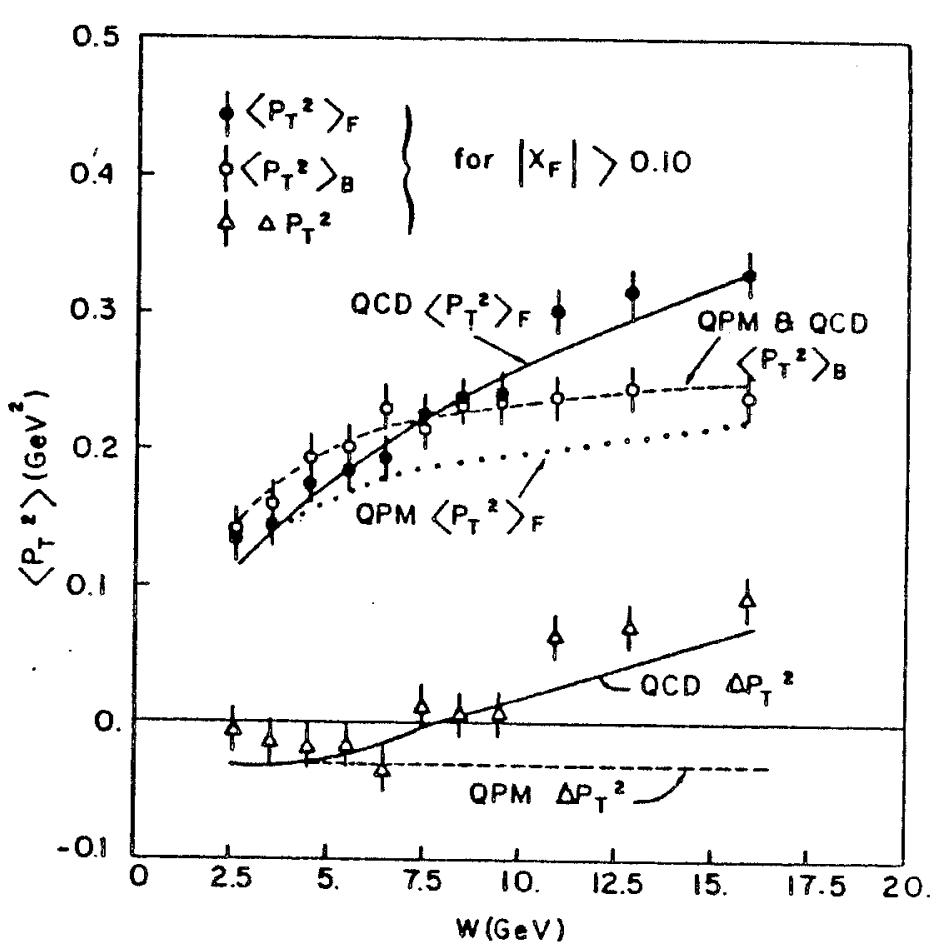

Figure $V-8(c)$ 

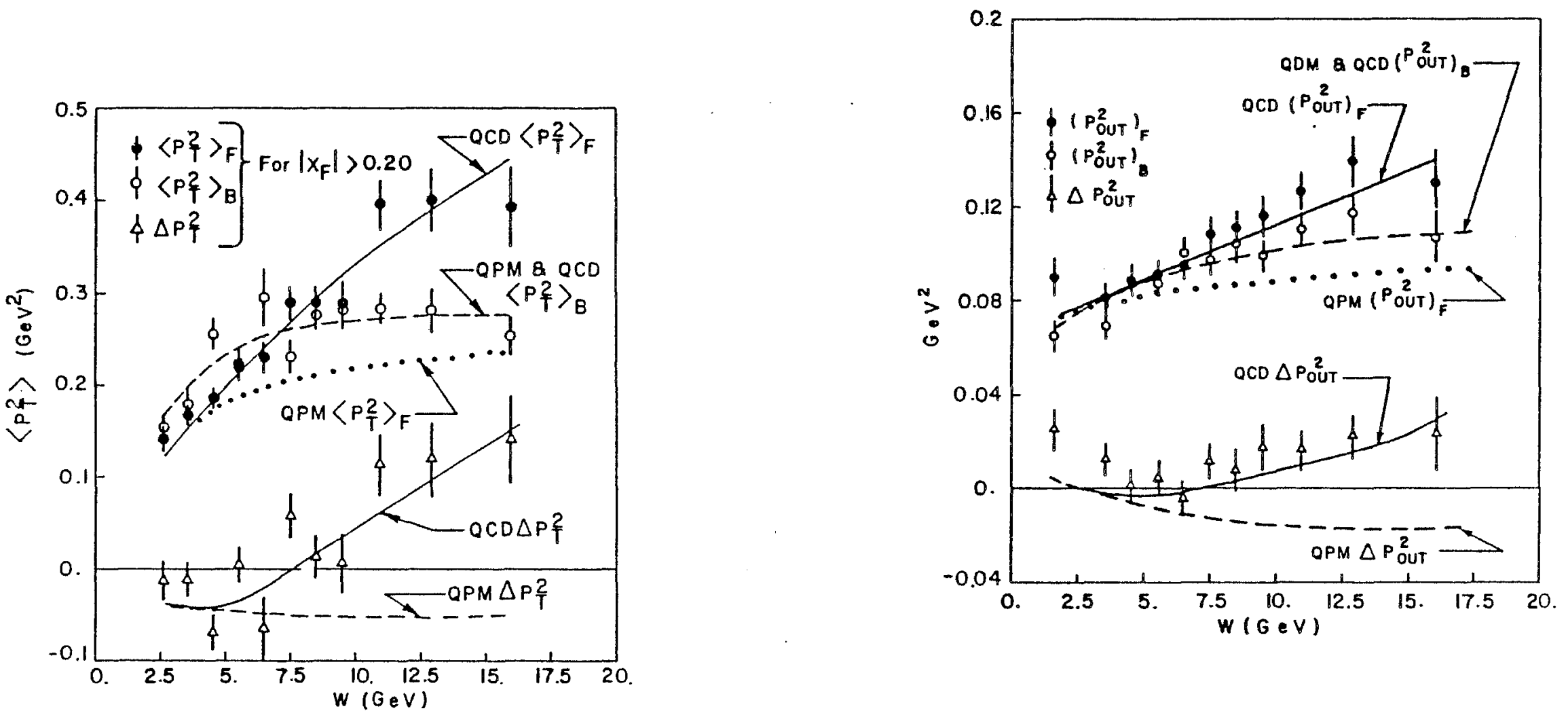

Figure V-9

Figure $V-8(d)$ 


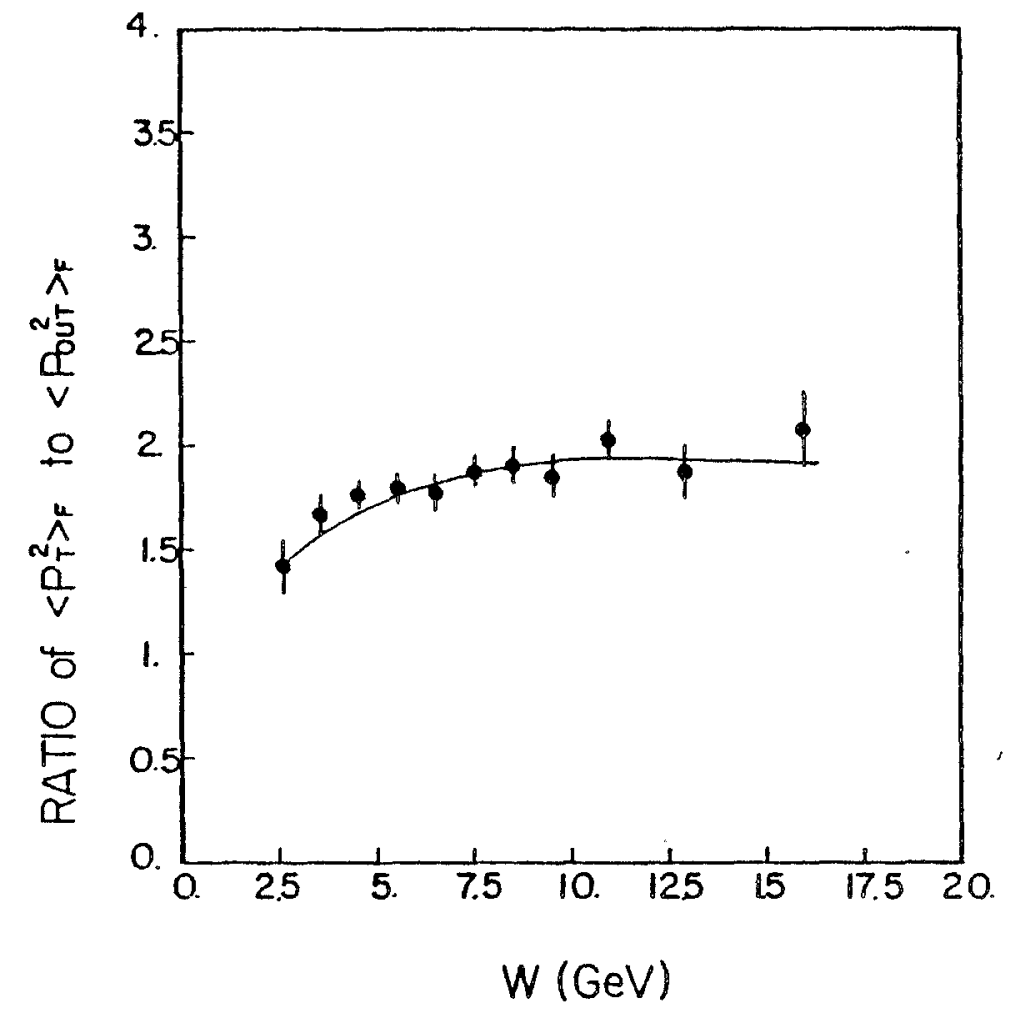

Figure V-10(a)

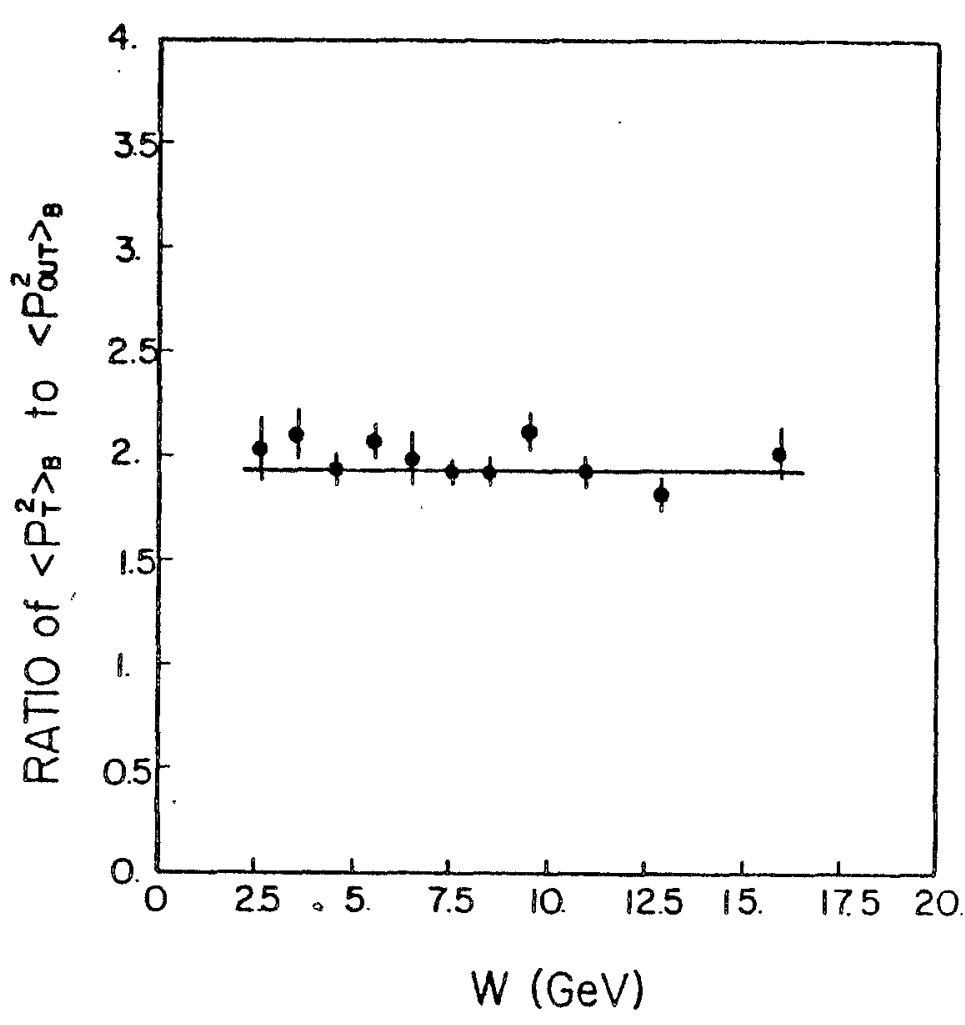

Figure $V-10(b)$ 


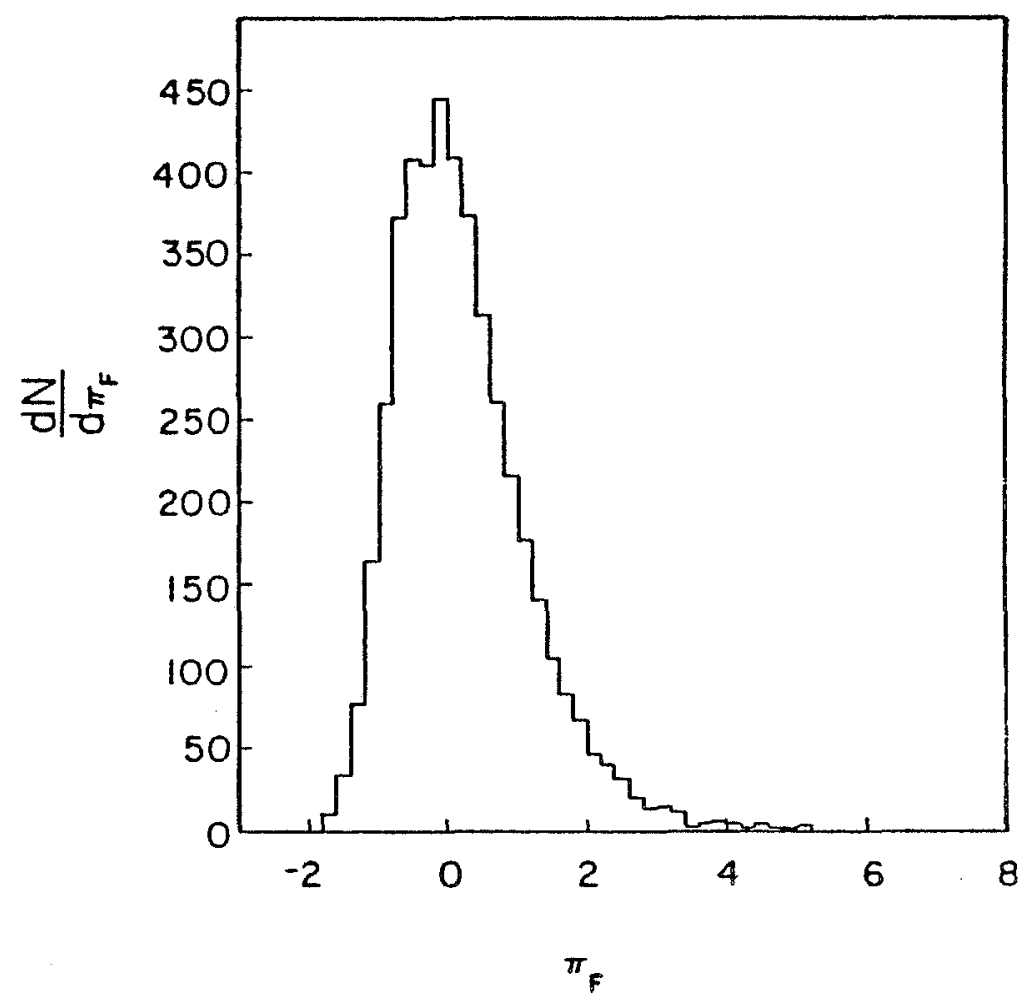

Figure $v-11(a)$

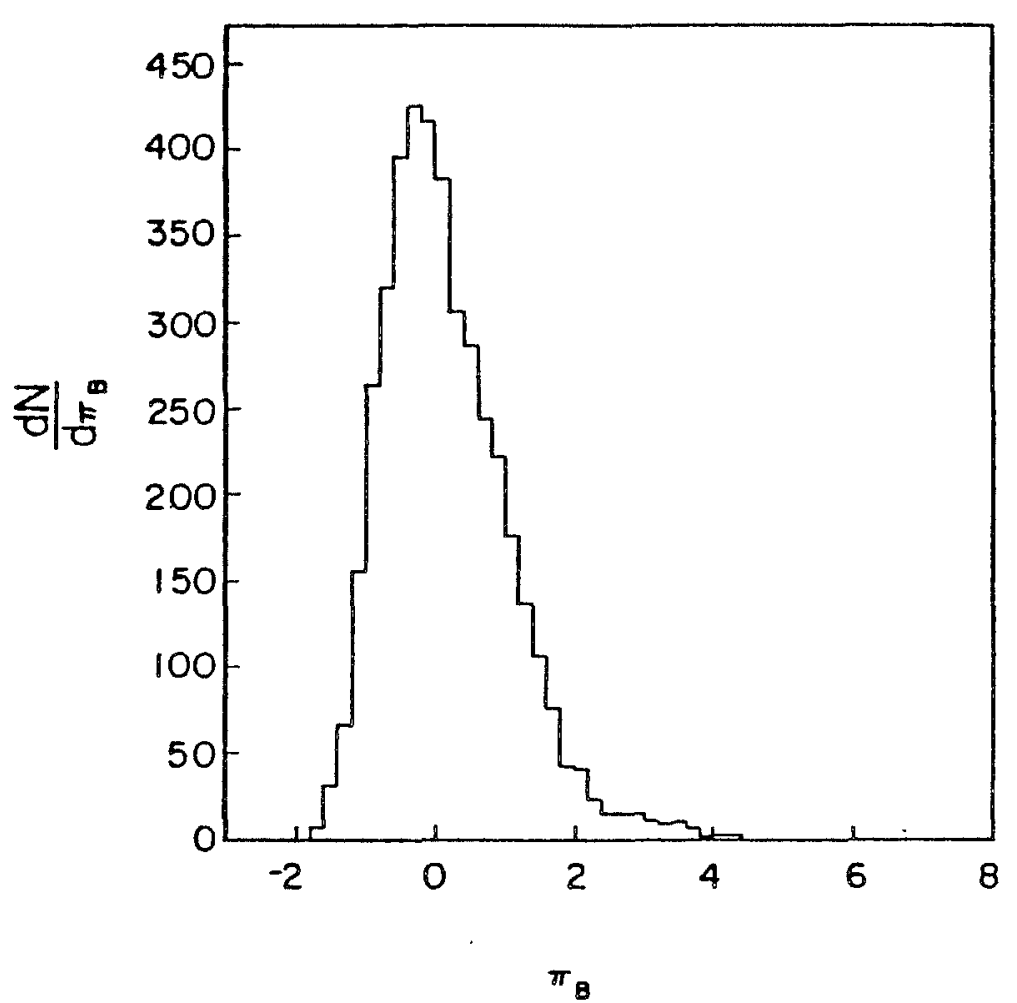

Figure $\mathrm{V}-11$ (b) 


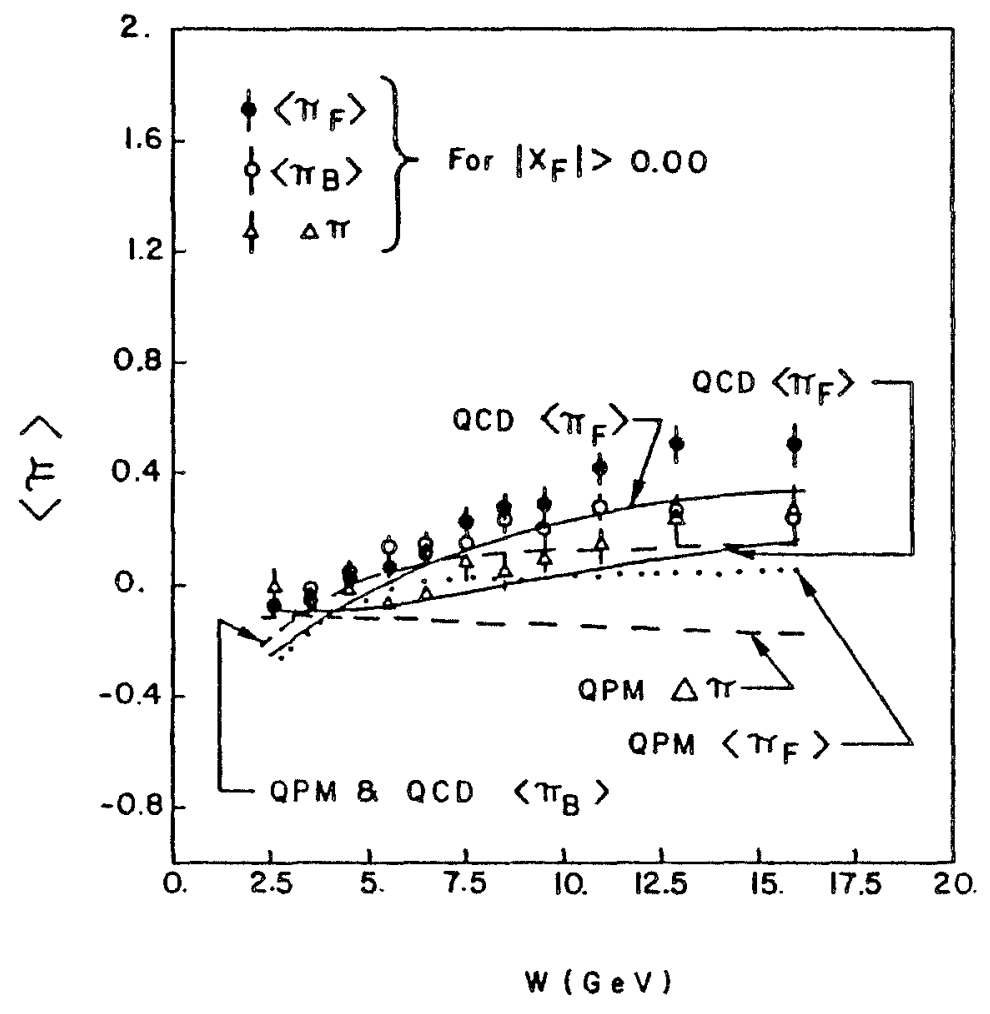

Figure $V-12(a)$

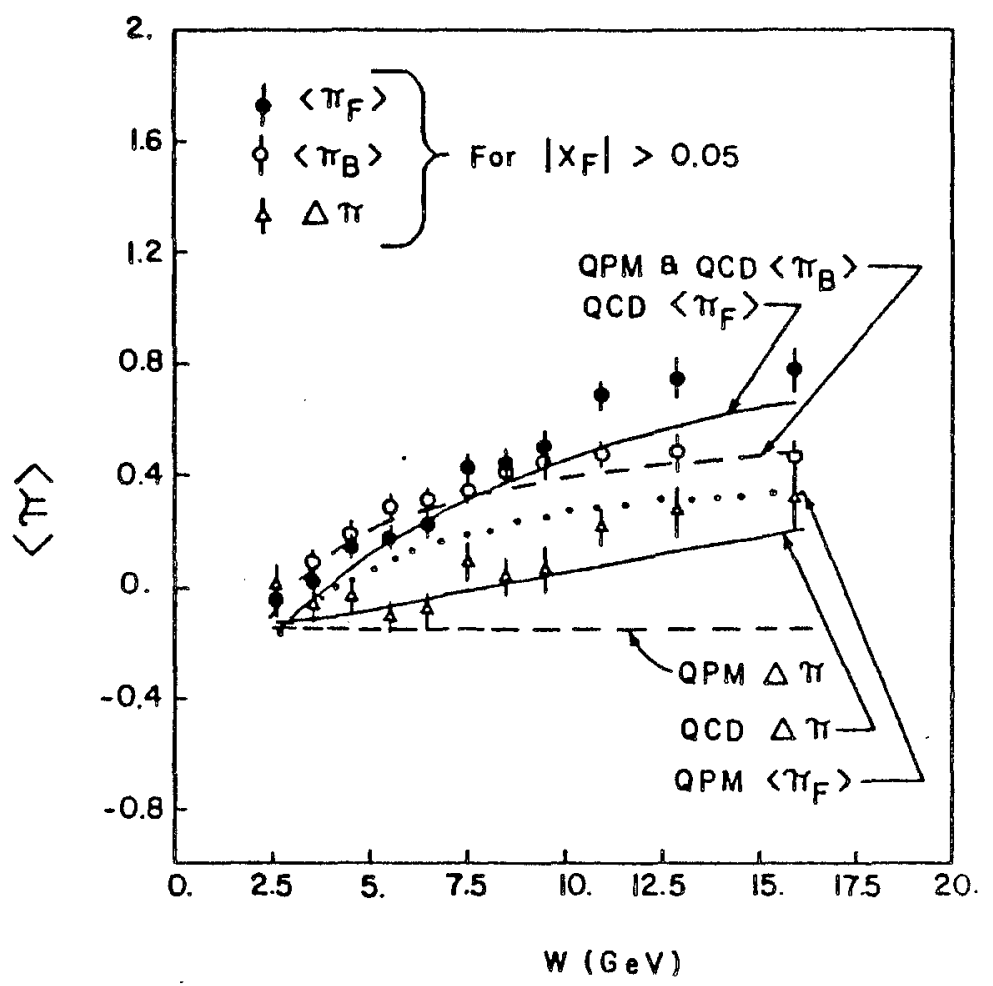

Figure $\mathrm{V}-12(\mathrm{~b})$ 


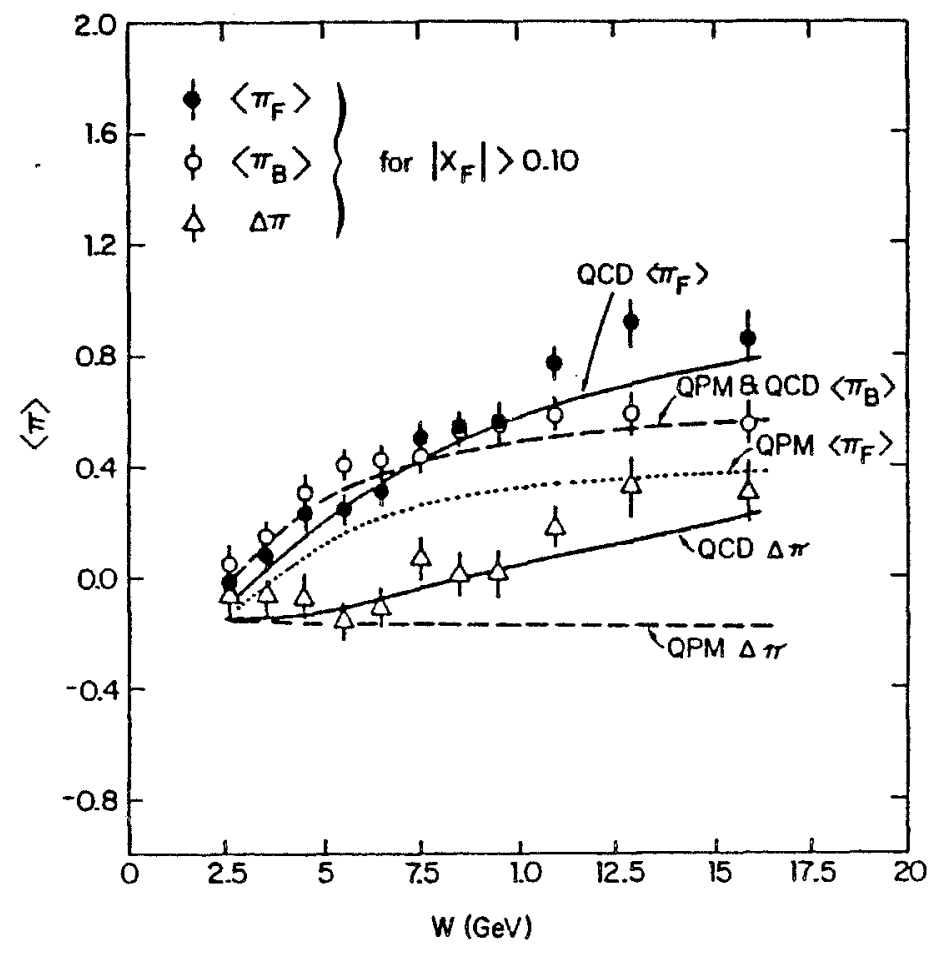

Figure $V-12(c)$

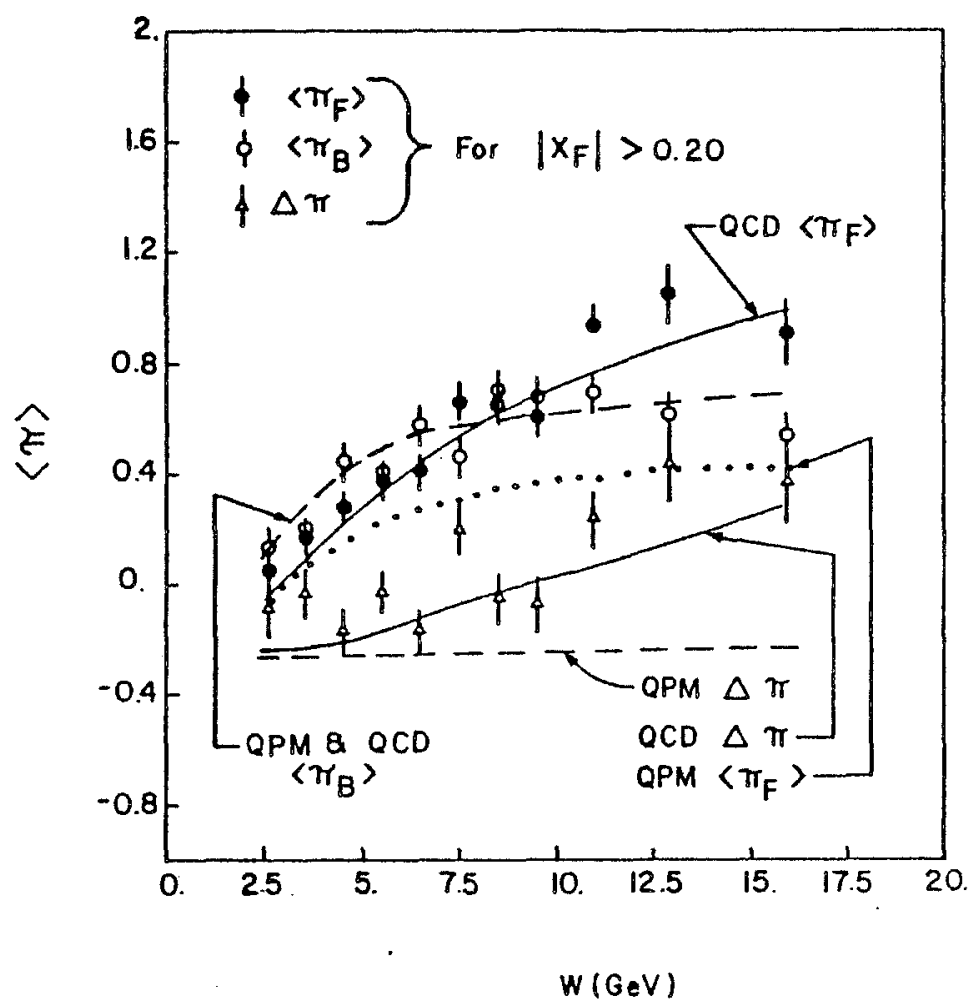

Figure $\mathrm{V}-12(d)$ 


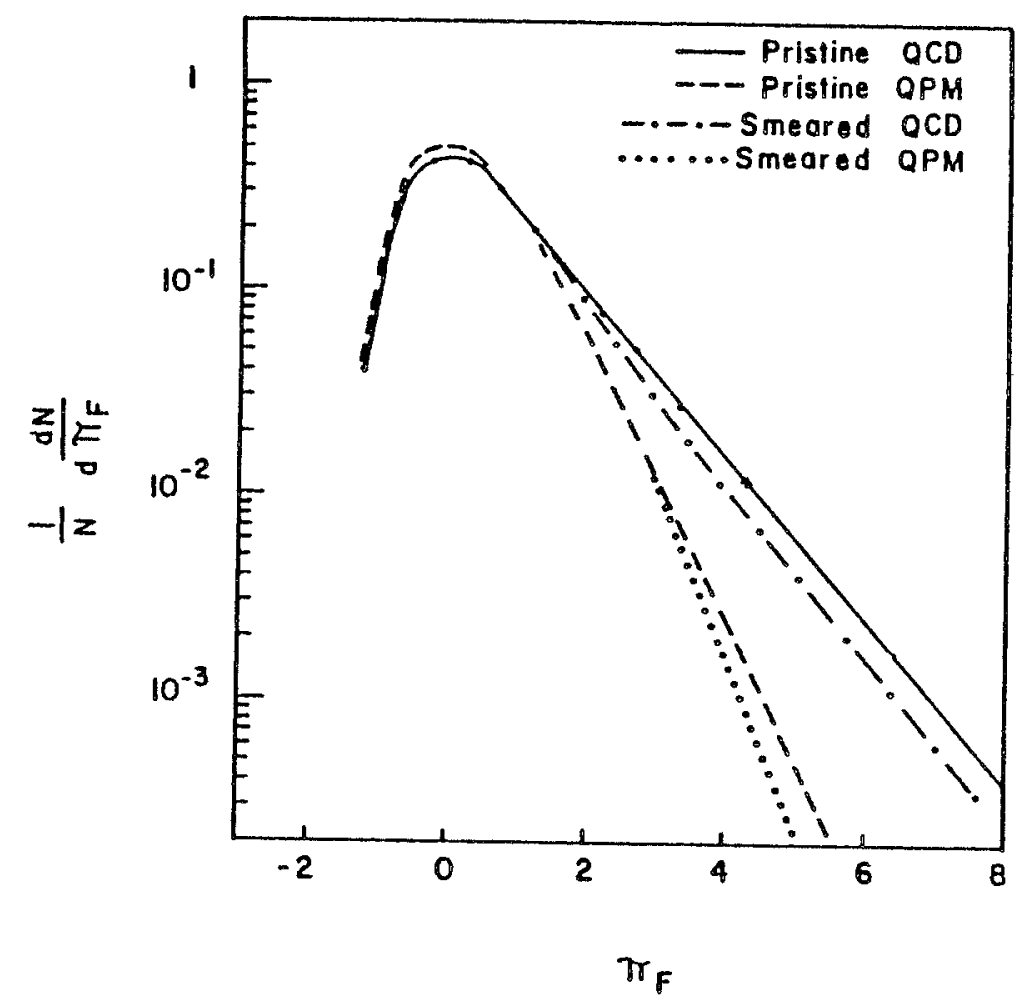

Figure $V-13(a)$

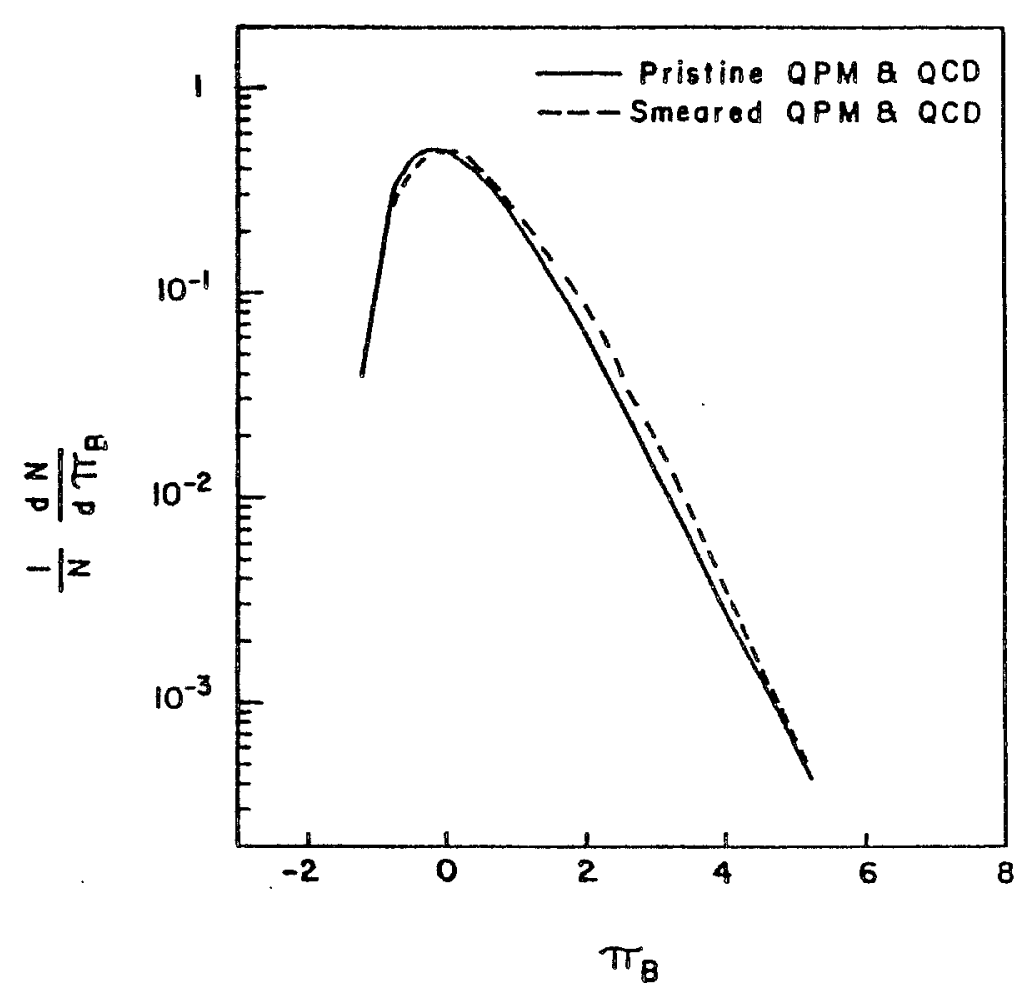

Figure $y-13(b)$ 

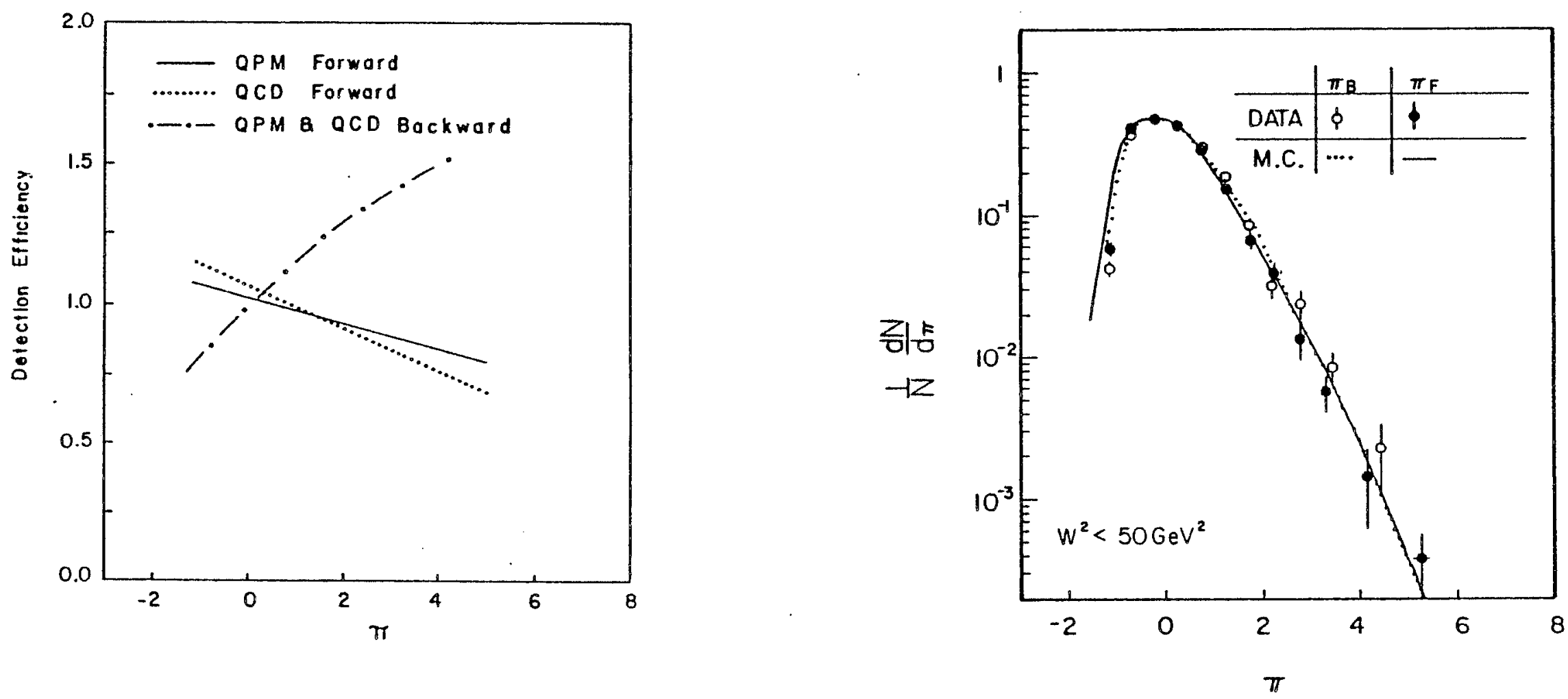

Figure V-14

Figure $V-15(a)$ 


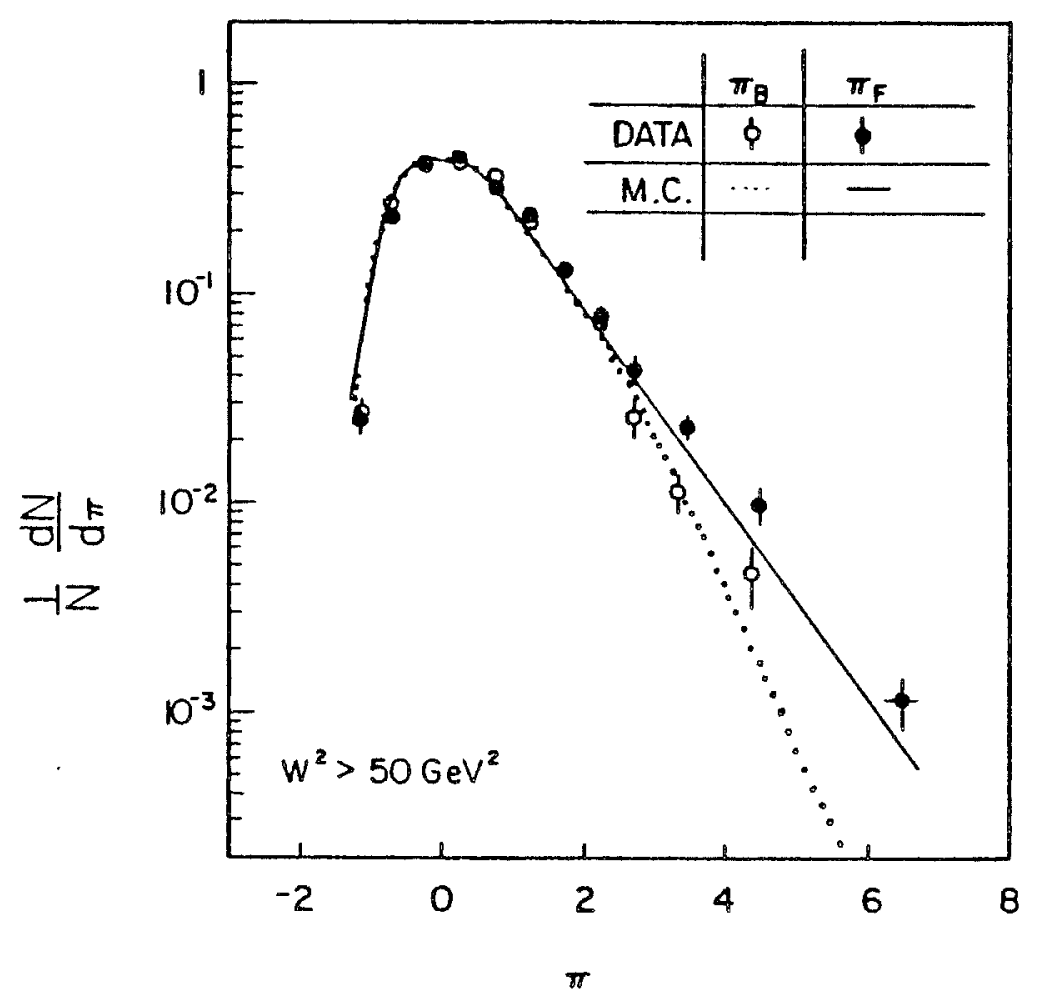

Figure $V-15(b)$

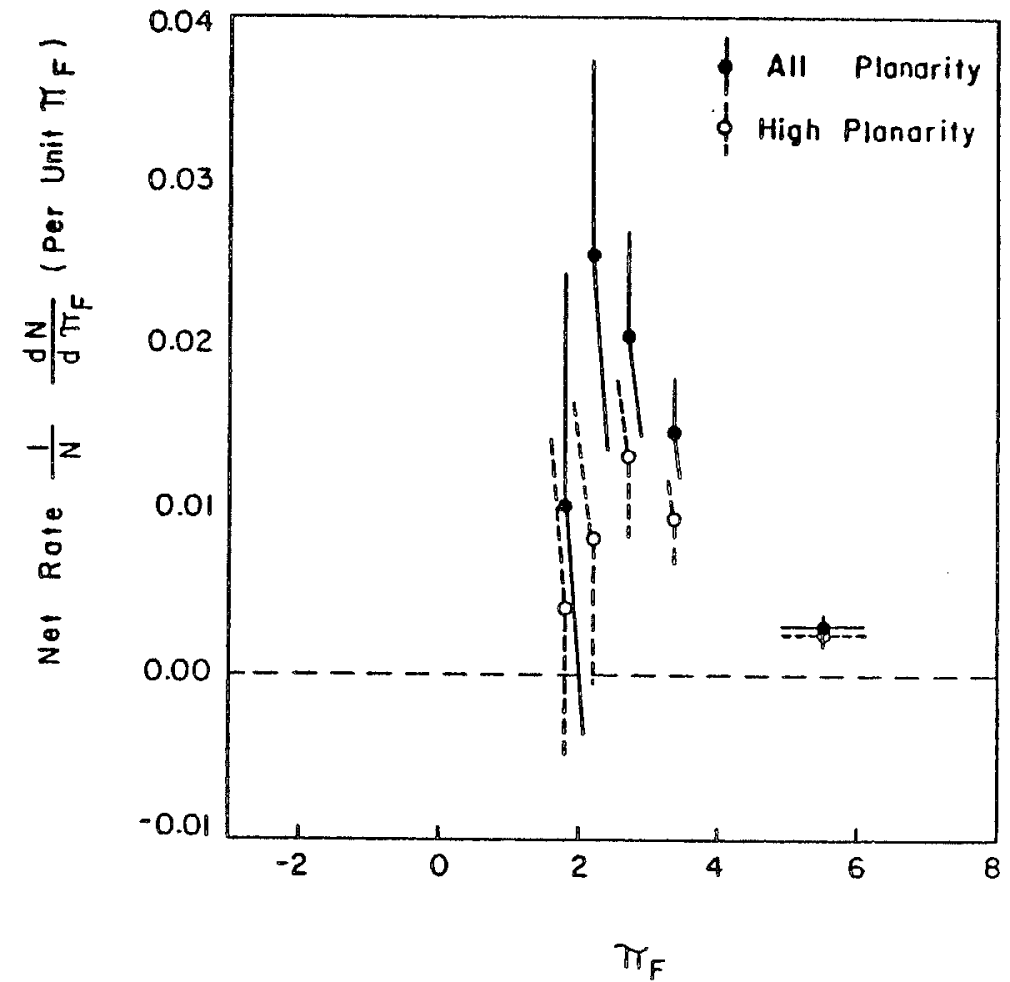

Figure $V-16$ 


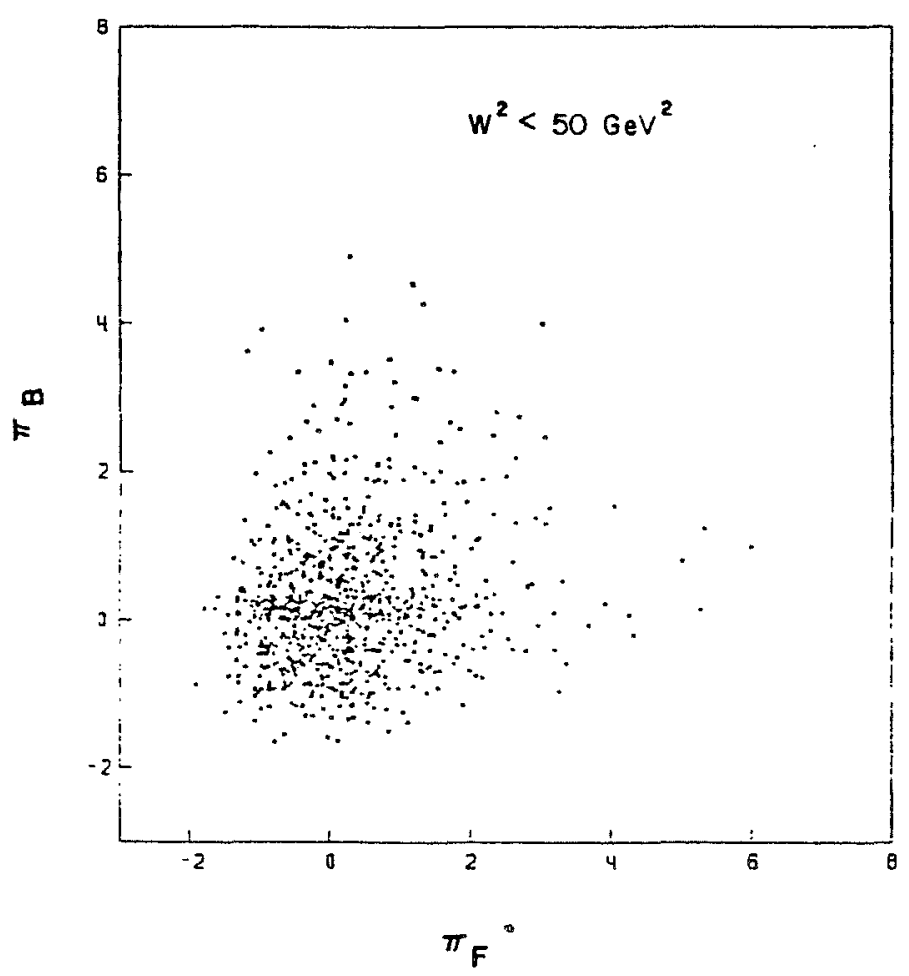

Figure $V-17(a)$

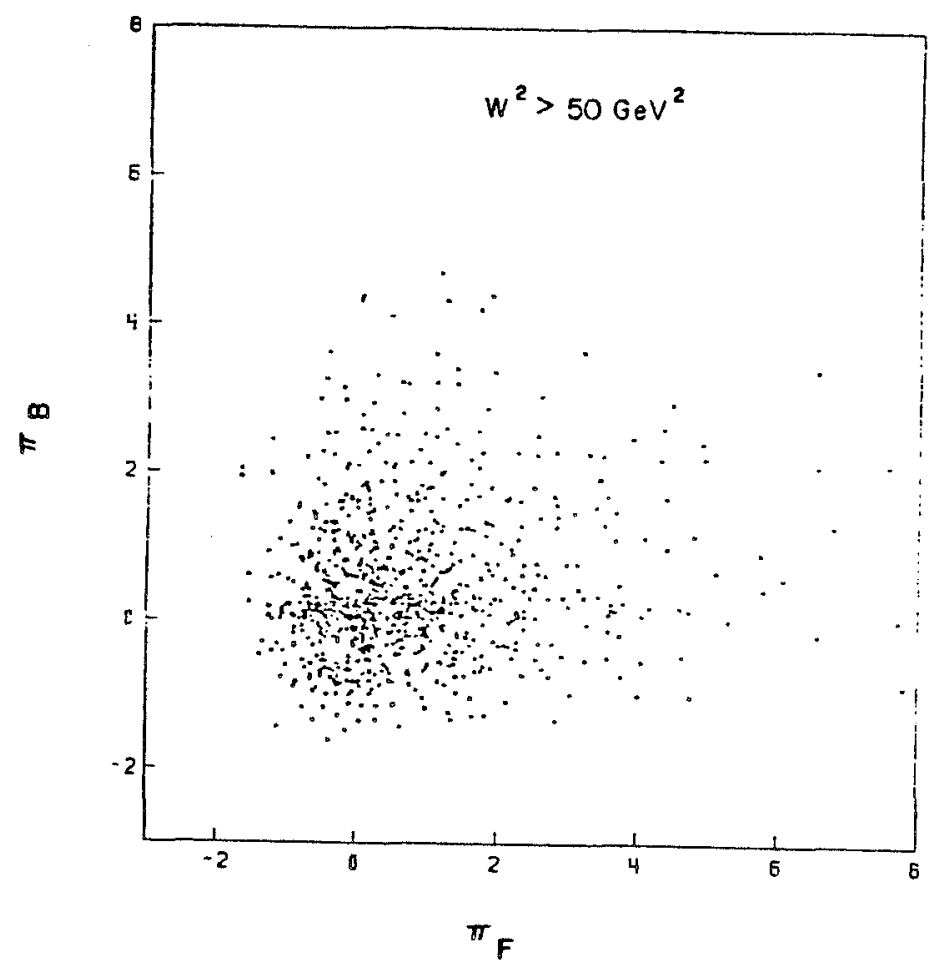

Figure $V-17(b)$ 


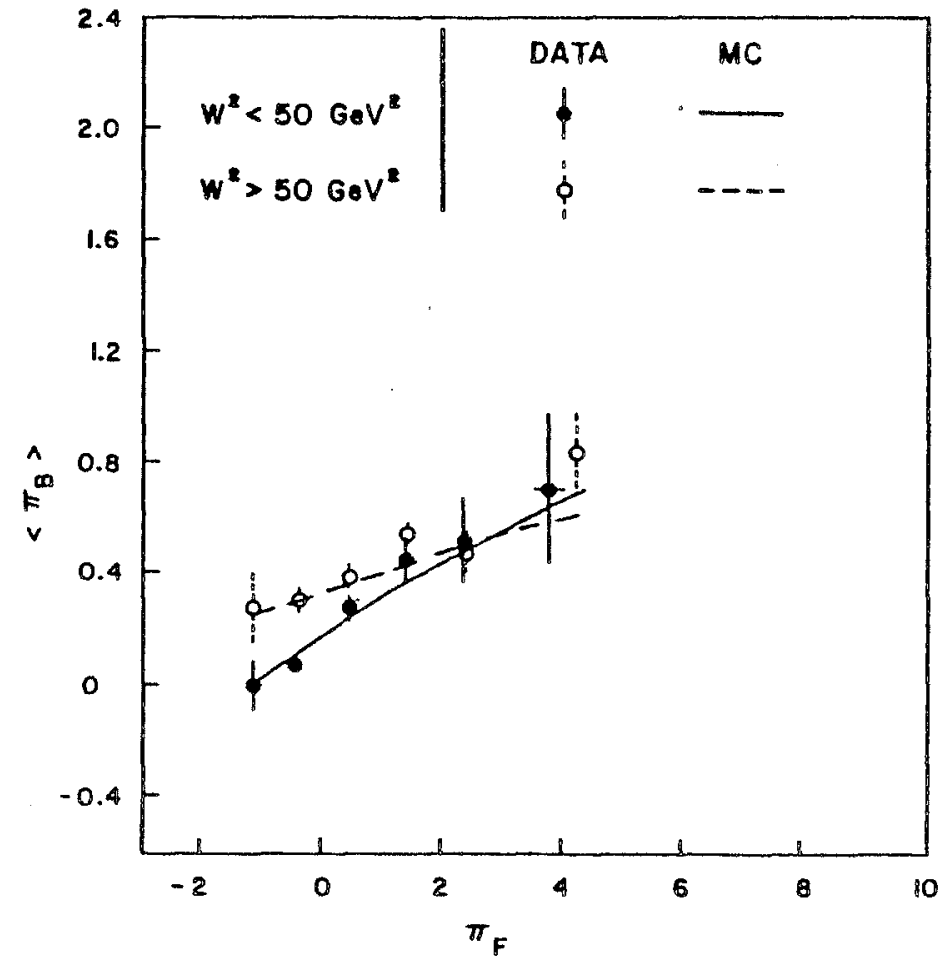

Figure $V-18$

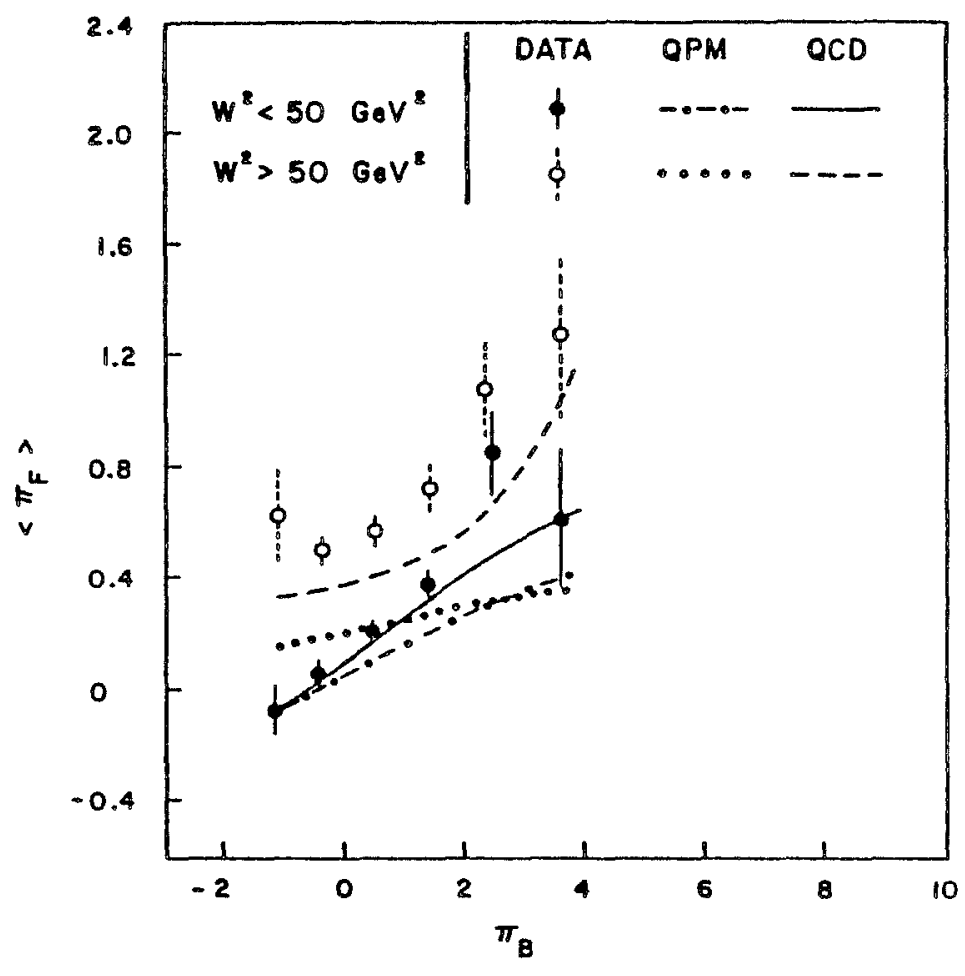

Figure $V-19$ 

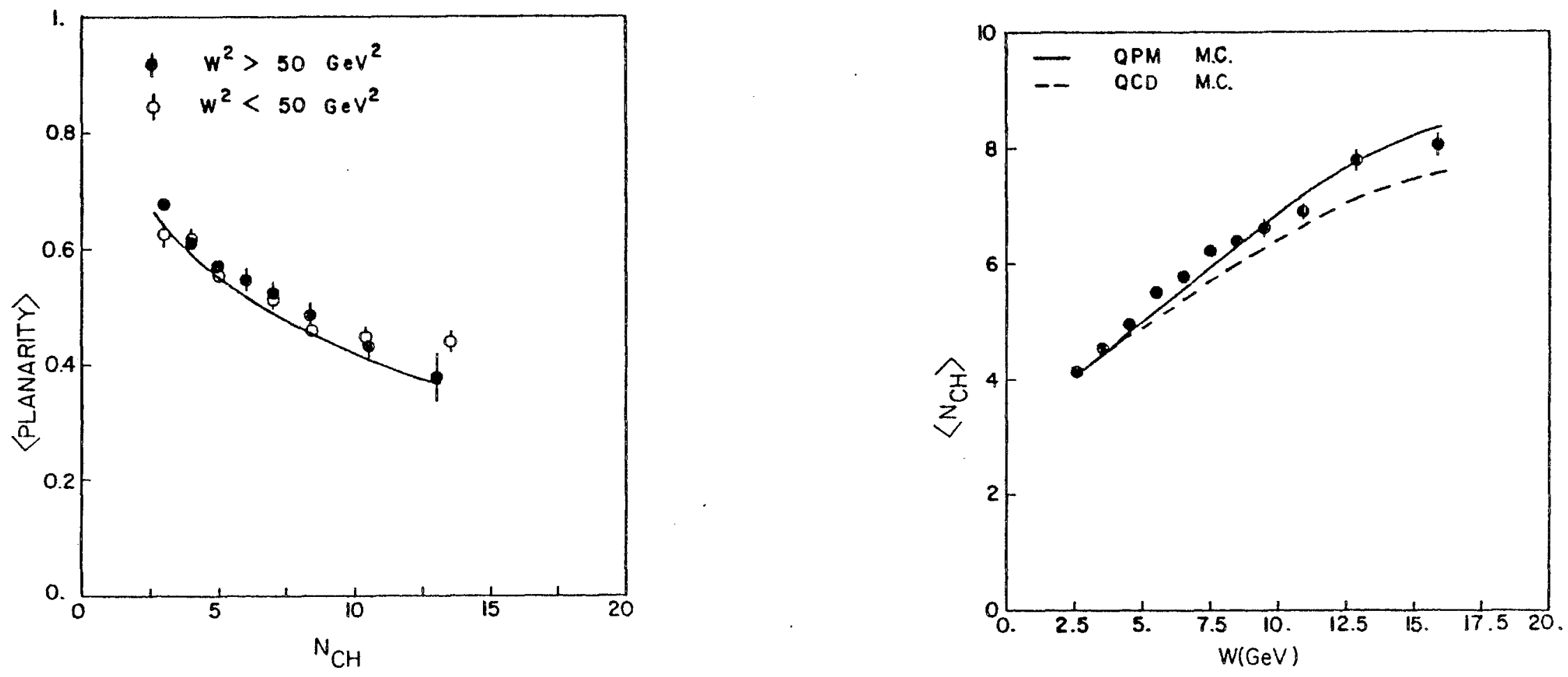

Figure $\mathrm{V}-20$

Figure V-21 


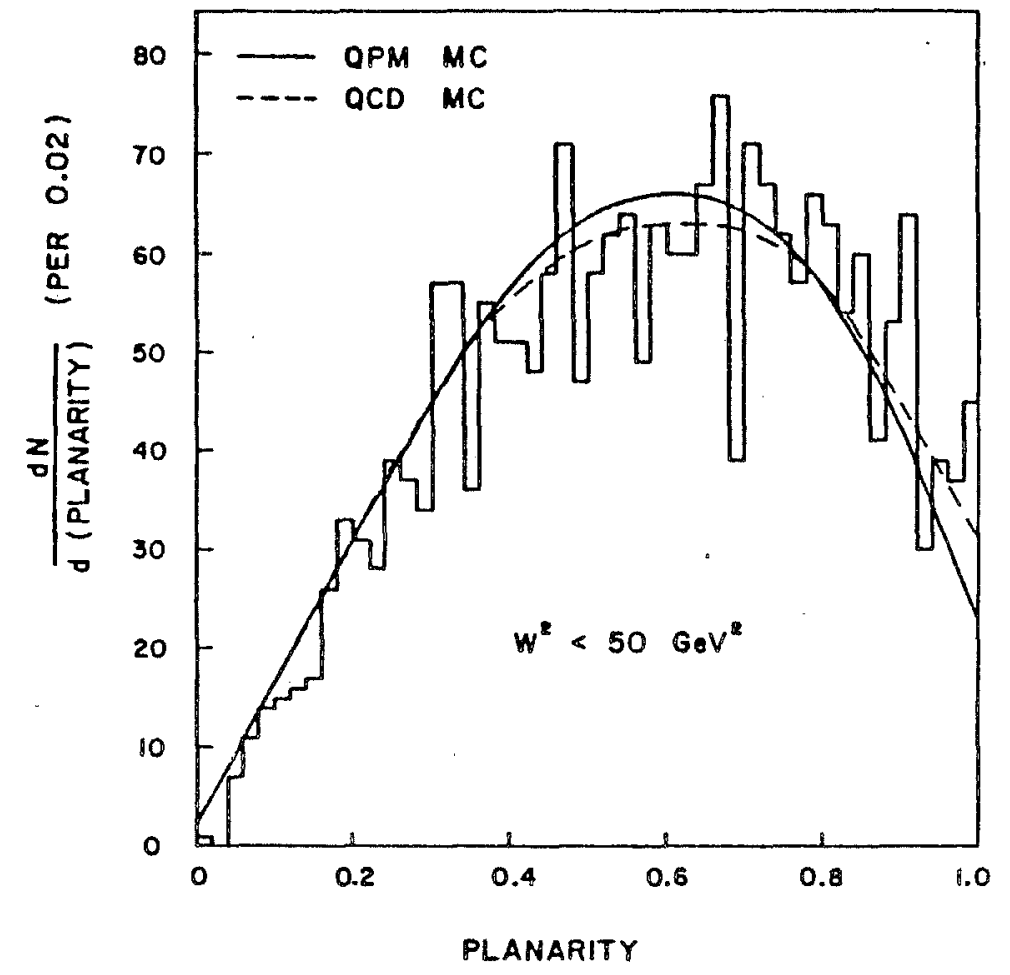

Figure $\mathrm{V}-22(\mathrm{a})$

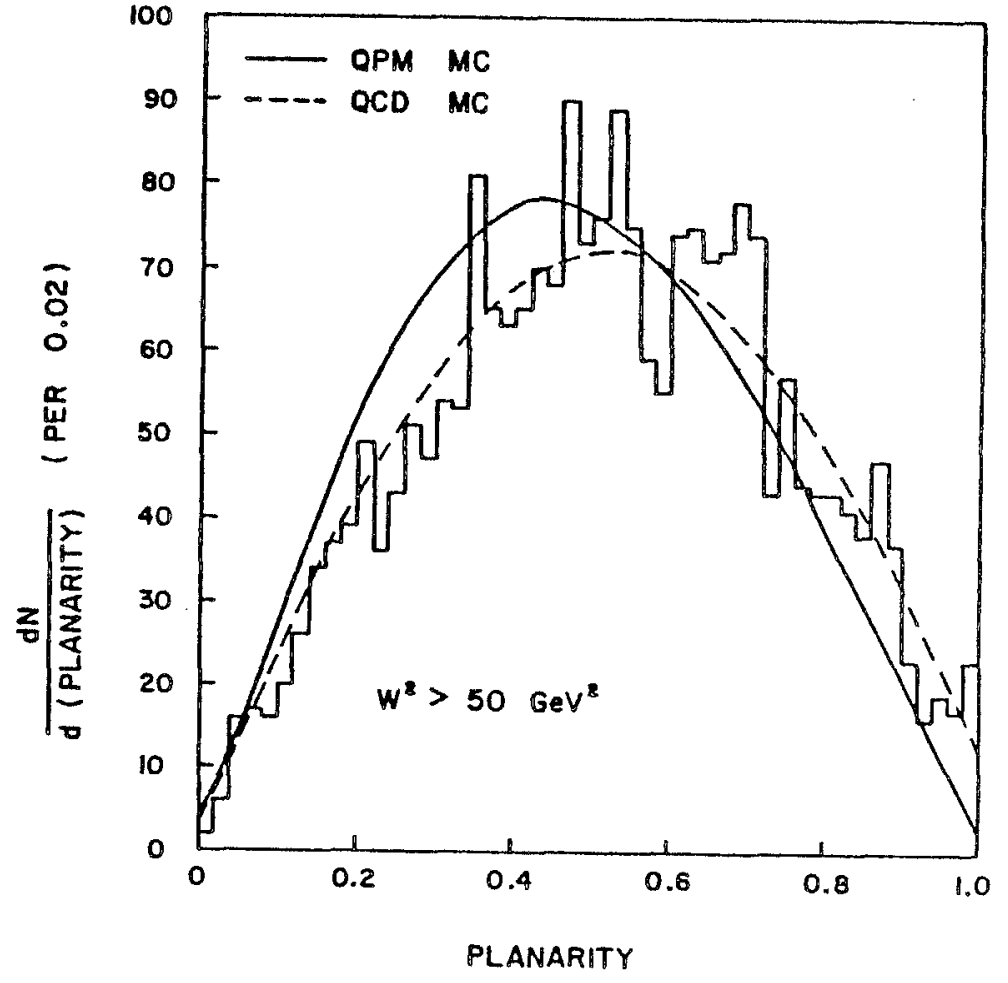

Figure $V-22(b)$ 


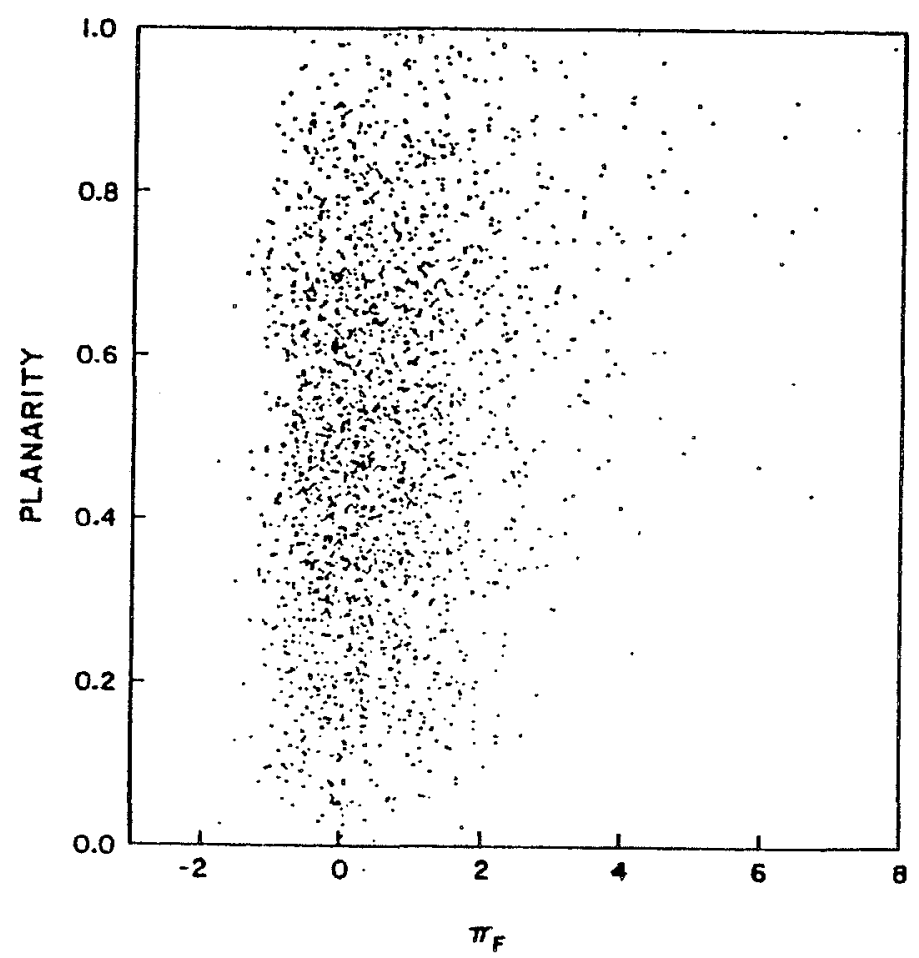

Figure $V-23$

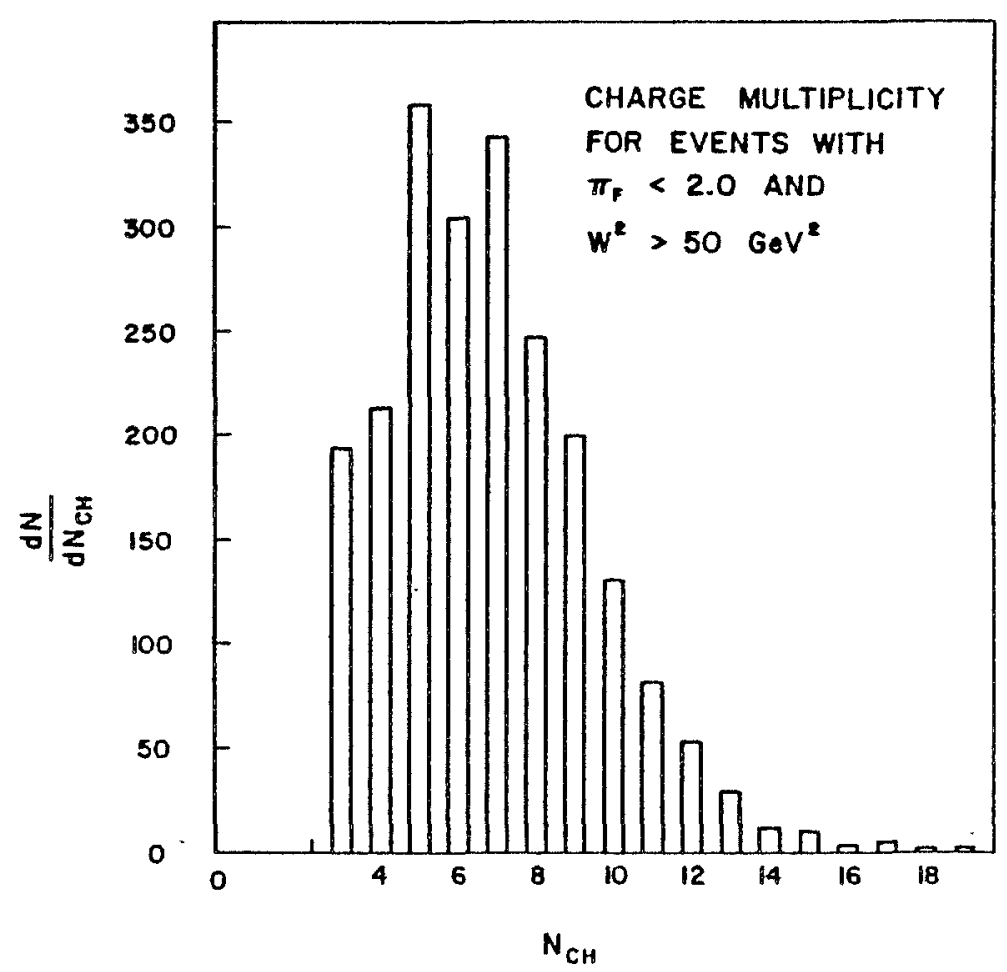

Figure $V-24(a)$ 


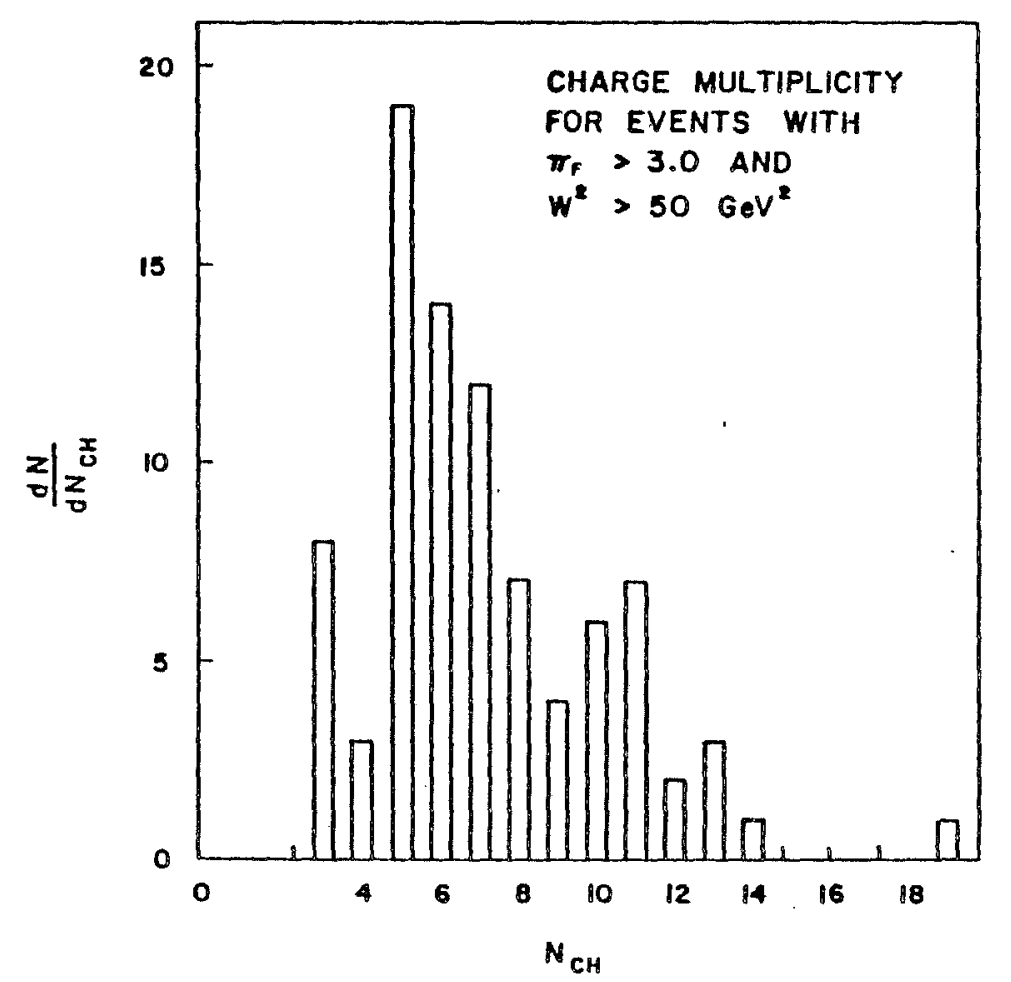

Figure $V-24(b)$

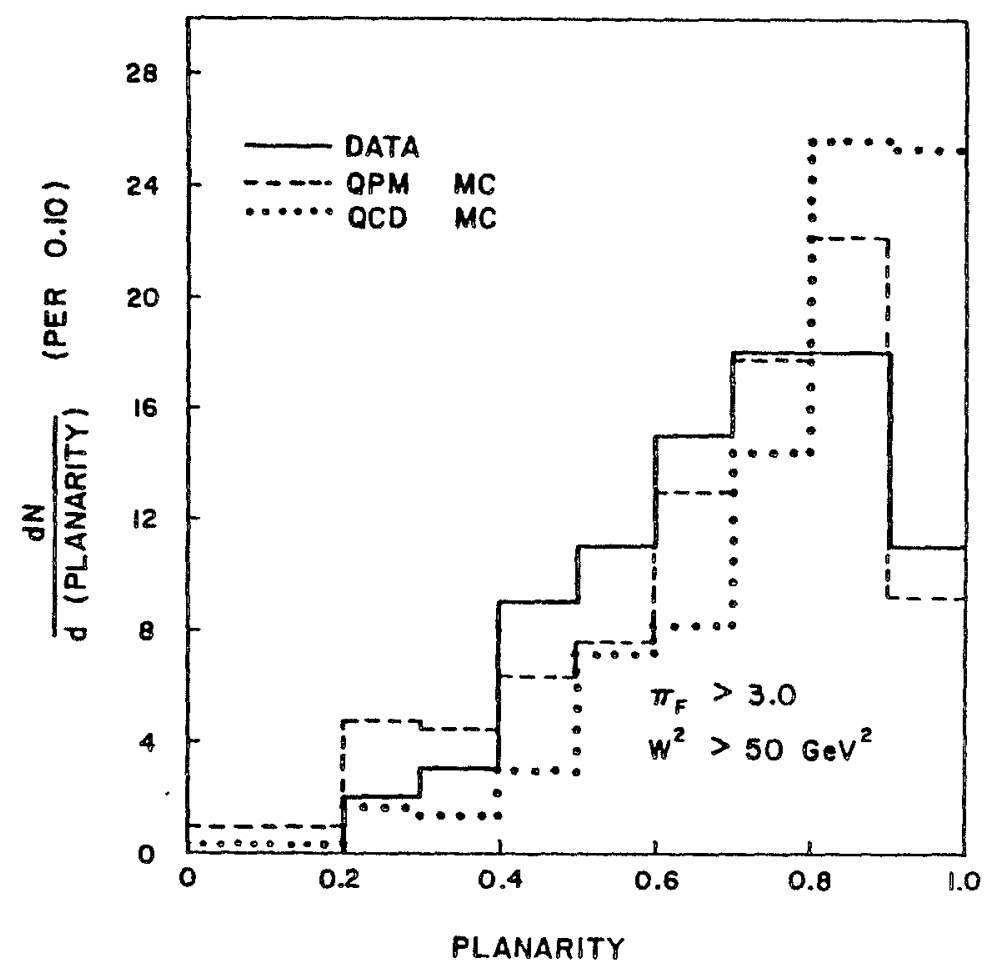

Figure $\mathrm{V}-25$ 


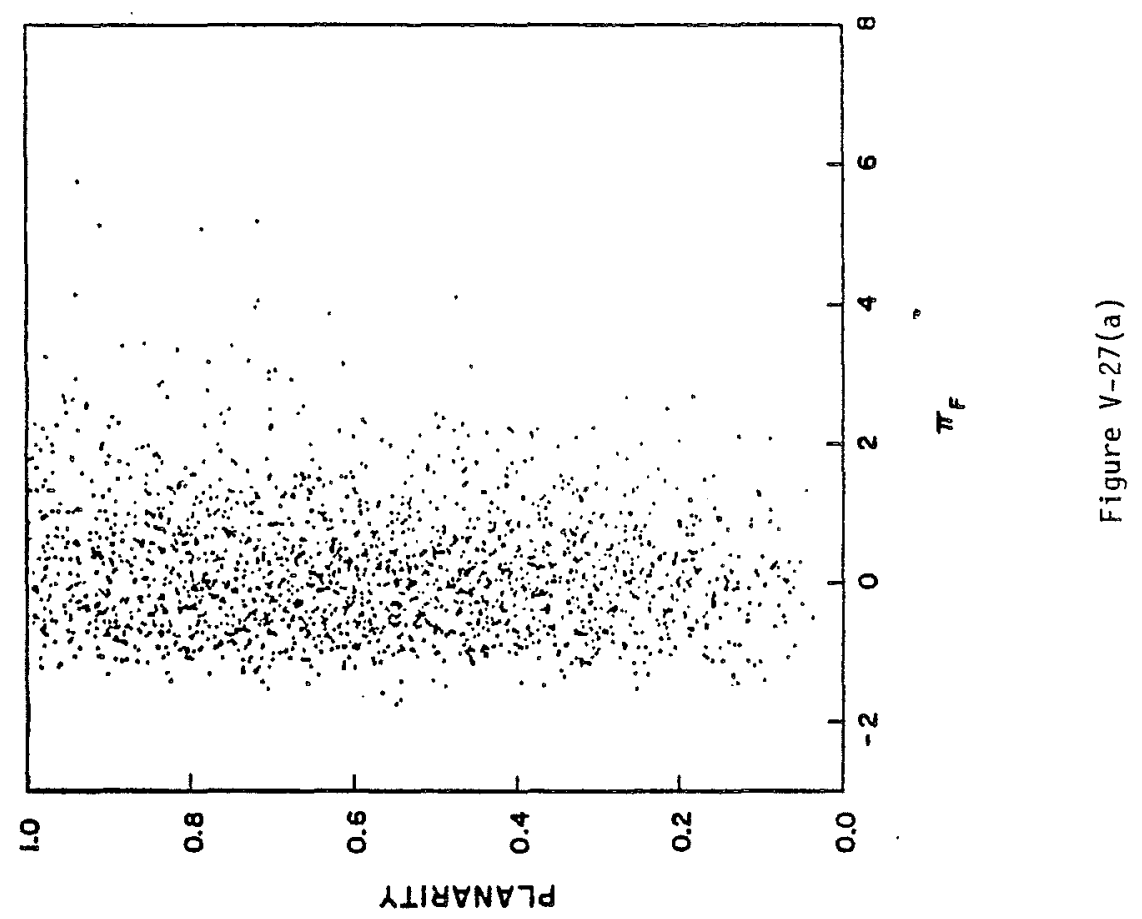

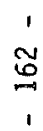

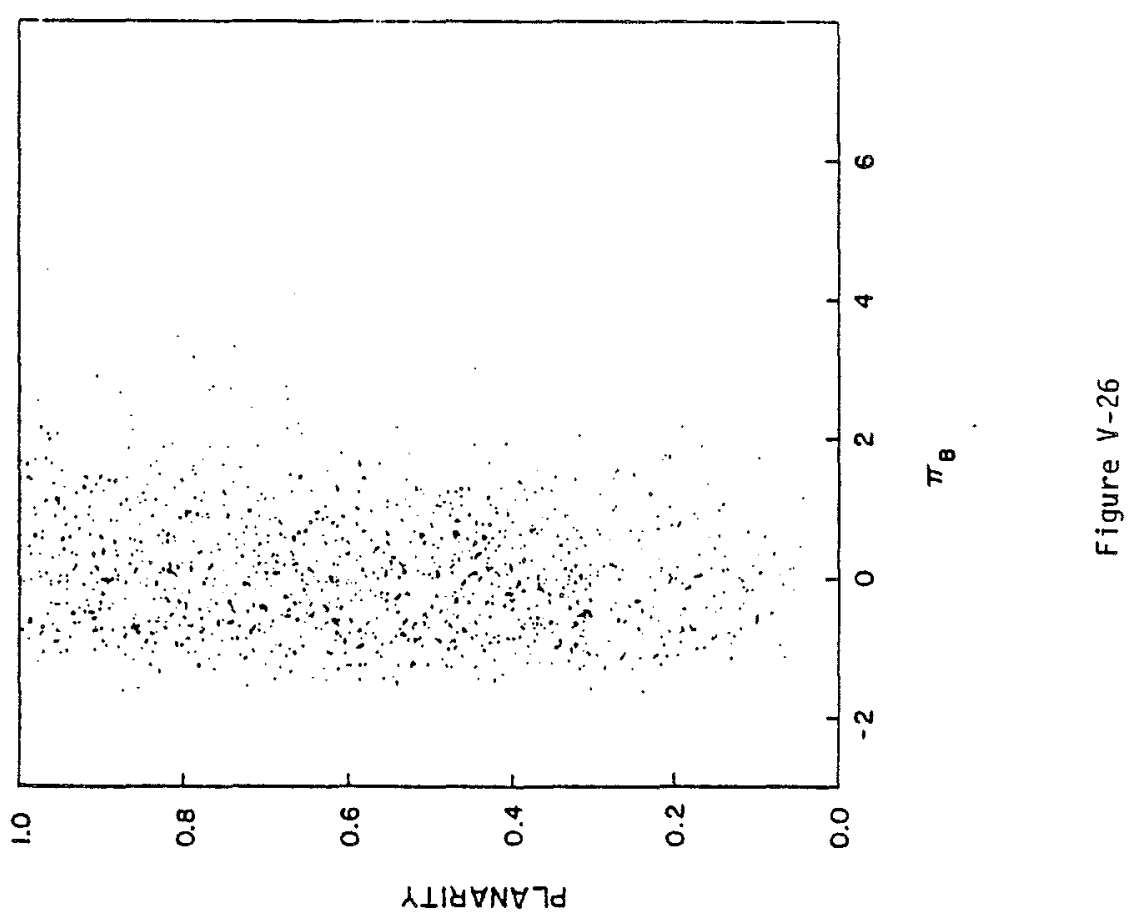




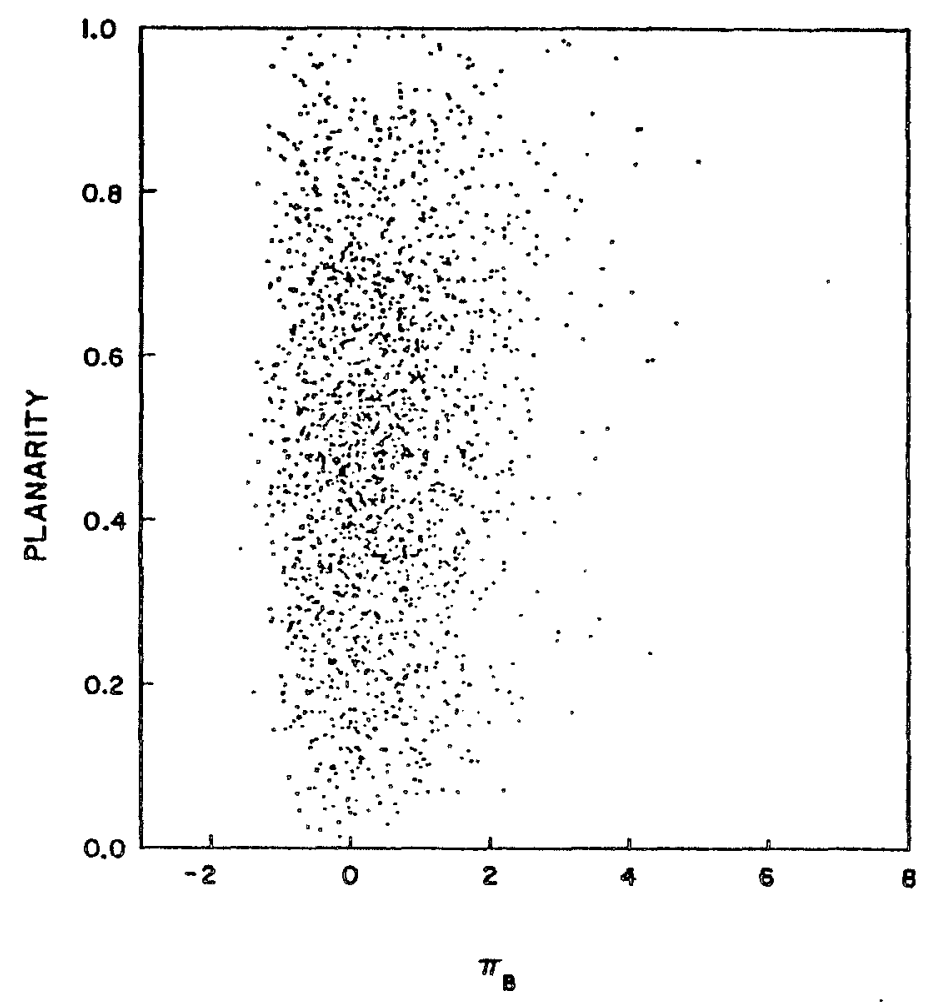

Figure $V-27(b)$

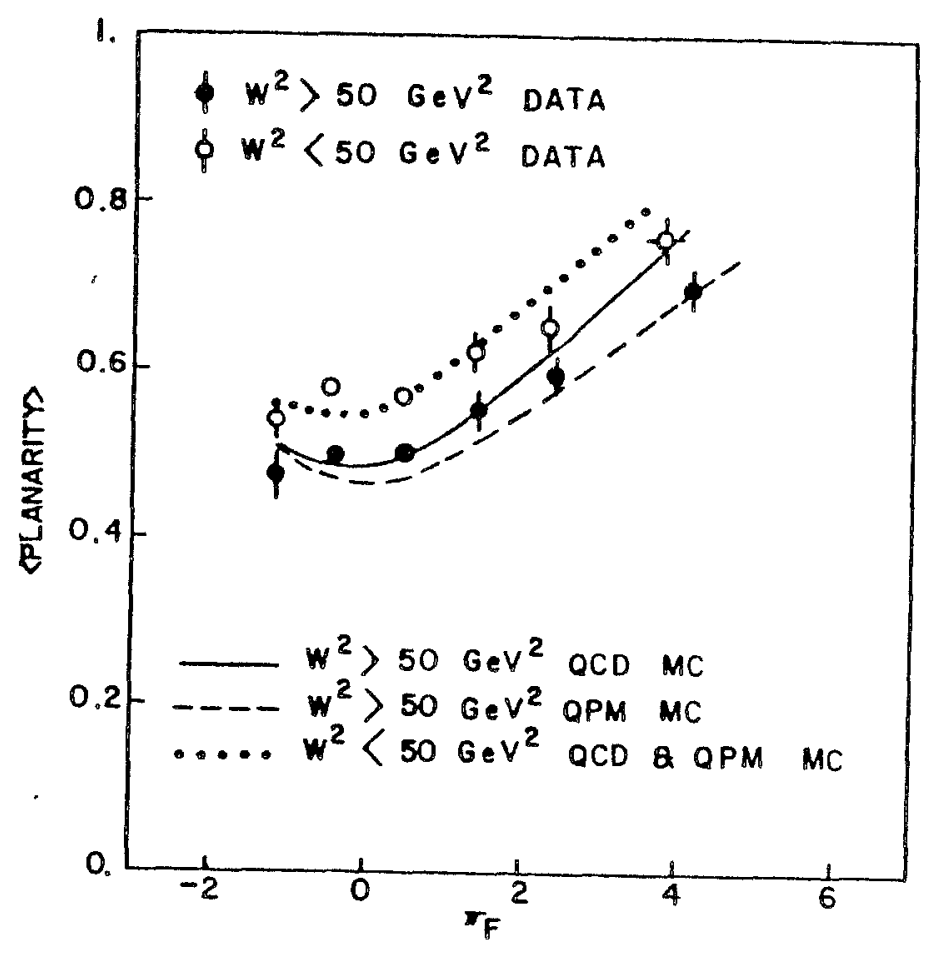

Figure $V-28(a)$ 


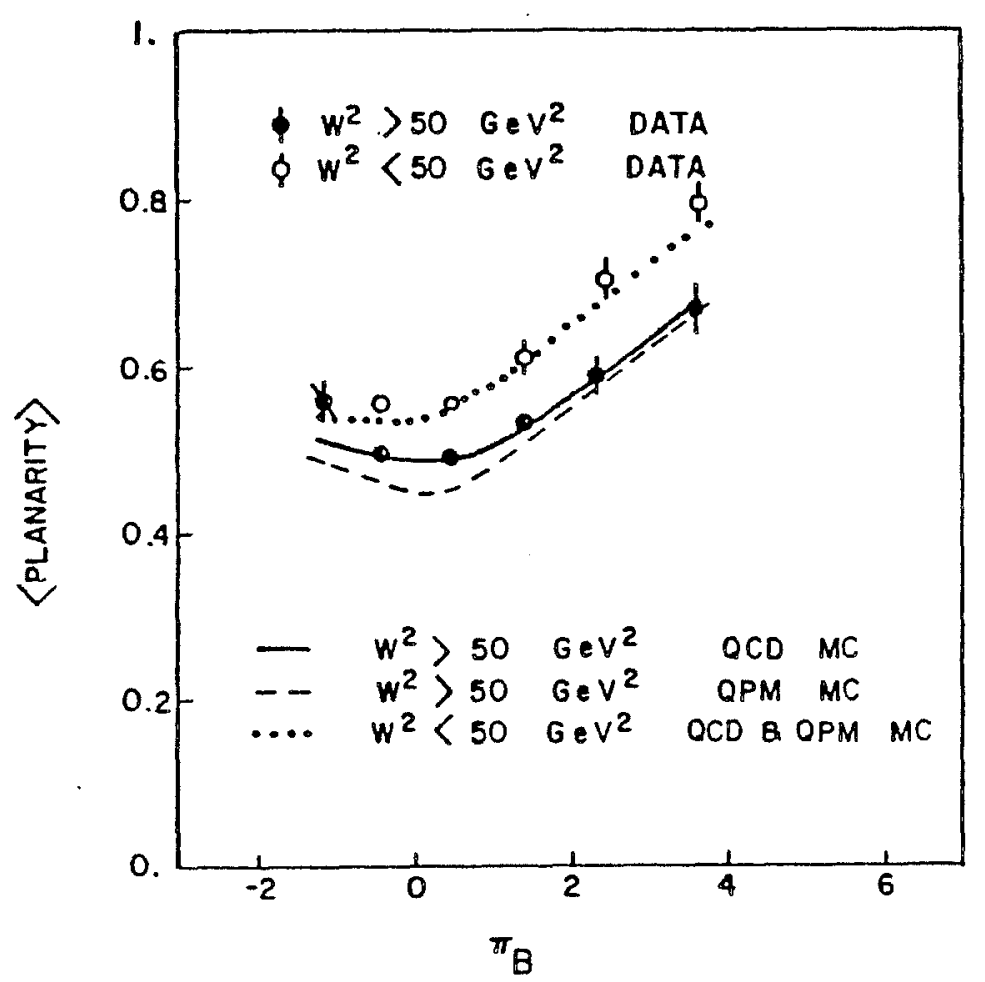

Figure $V-28(b)$

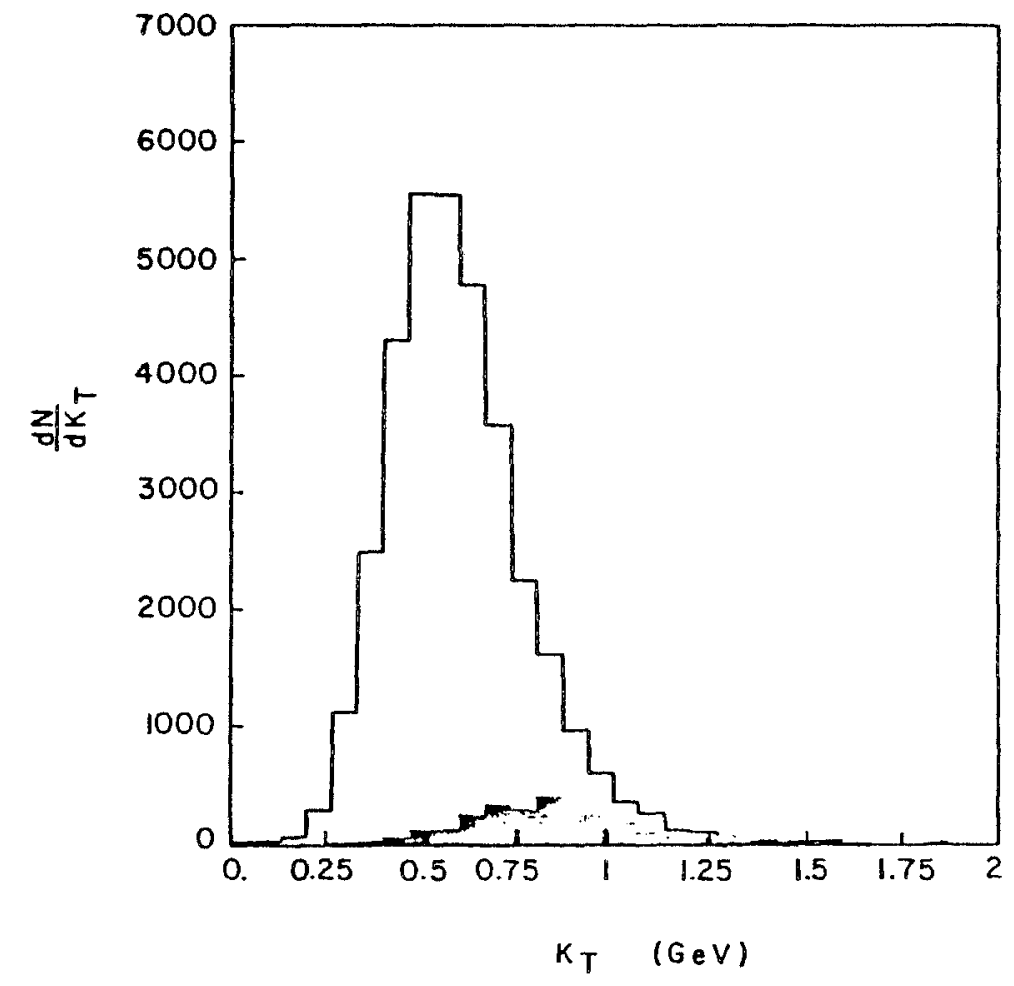

Figure V-29 
1
$g$
1

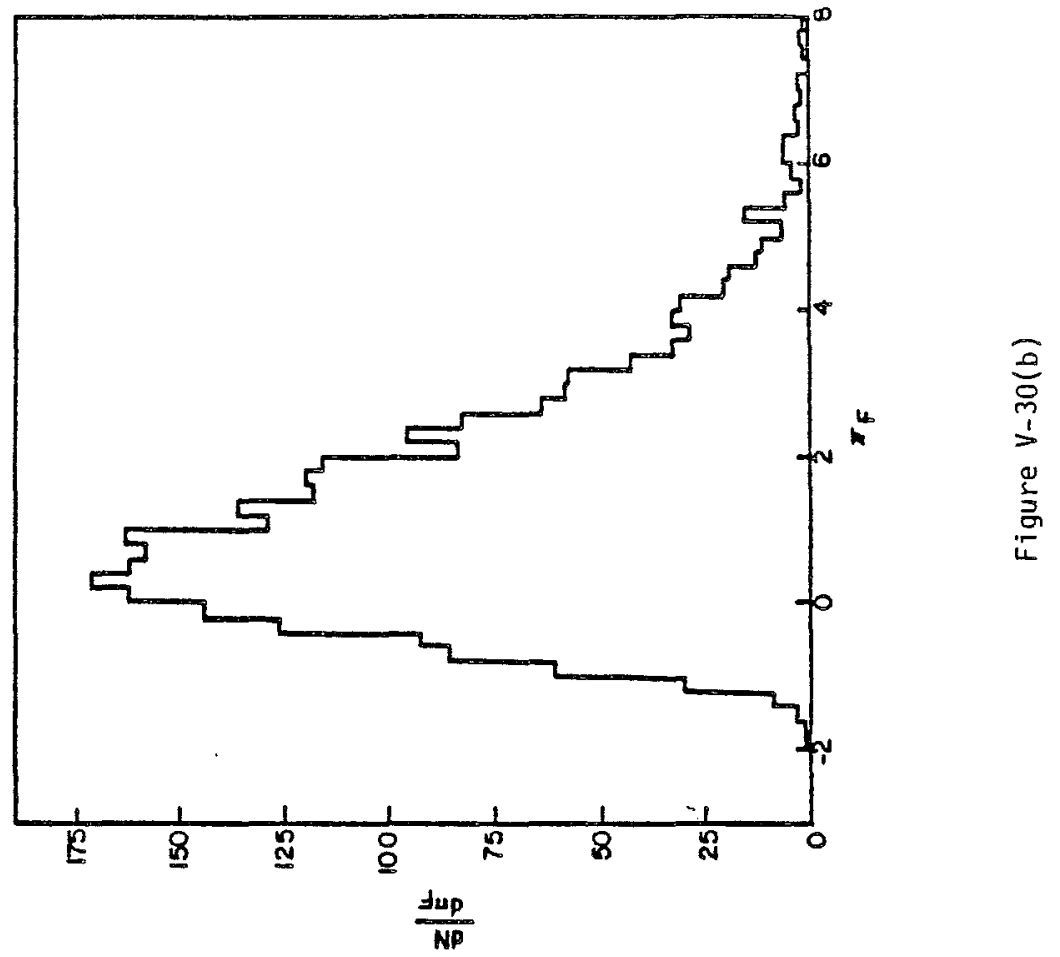

$\stackrel{\infty}{0}$

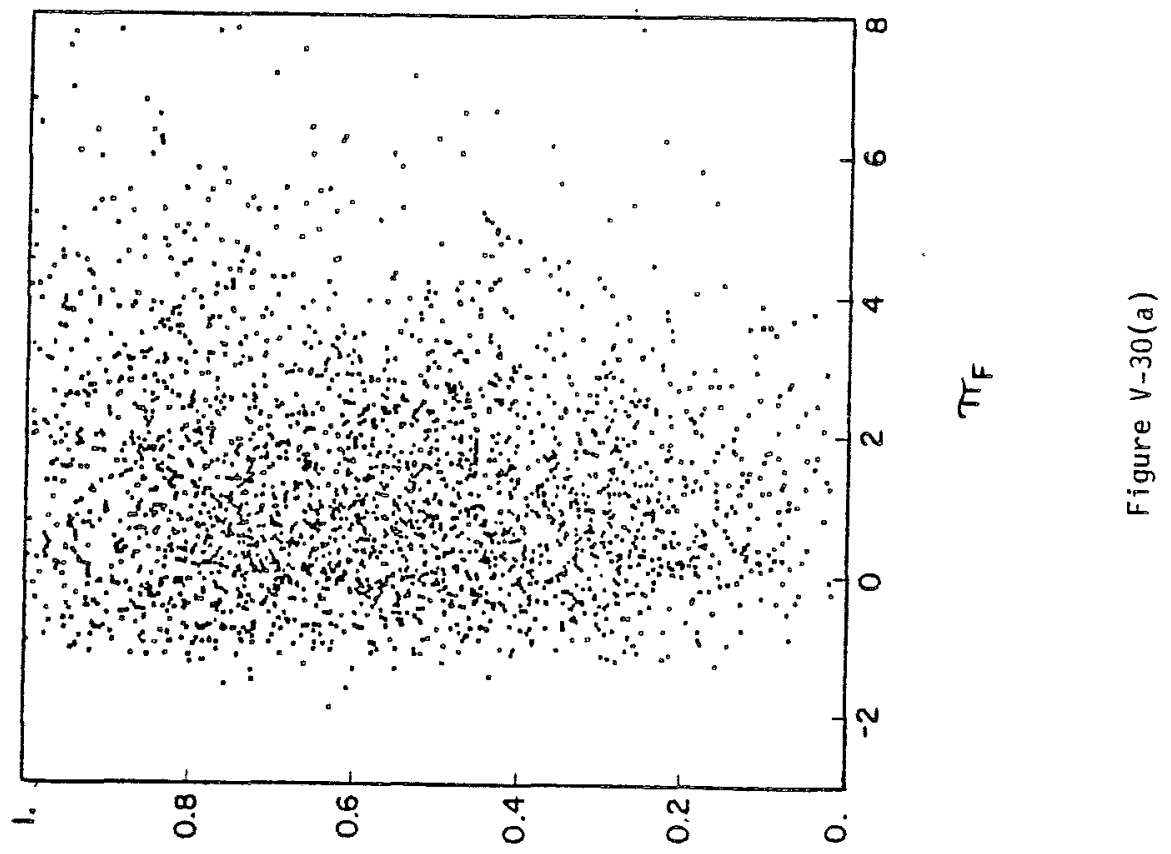




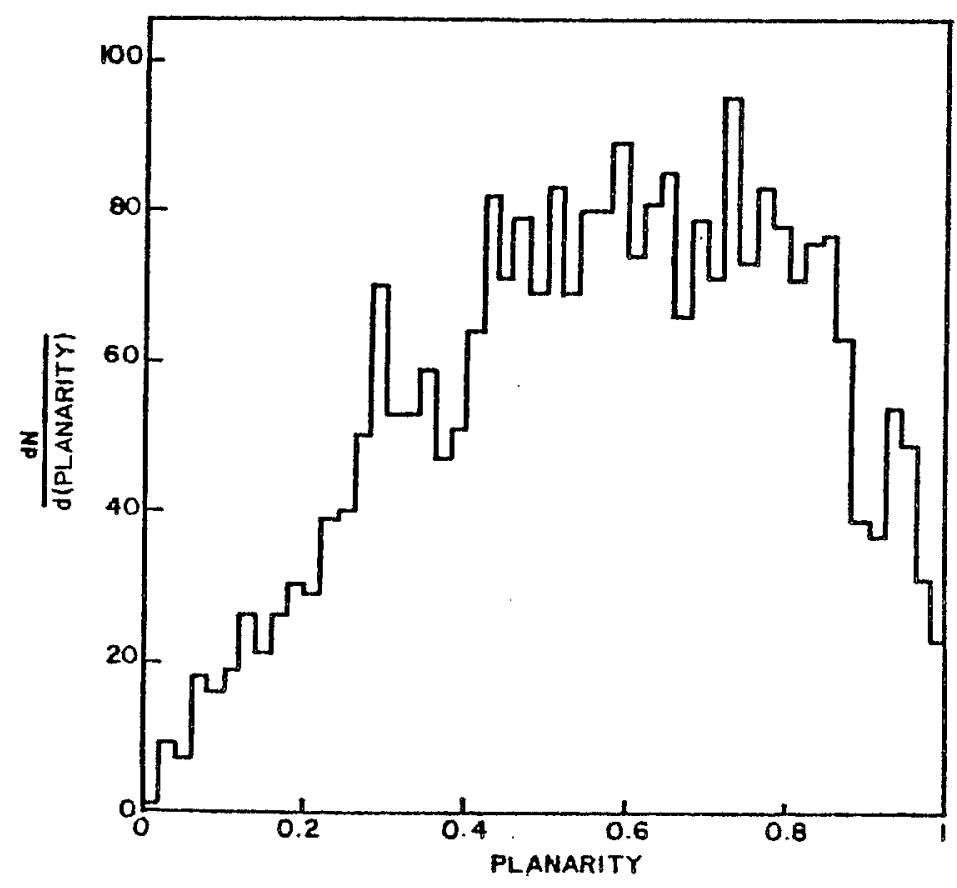

Figure $V-30(c)$

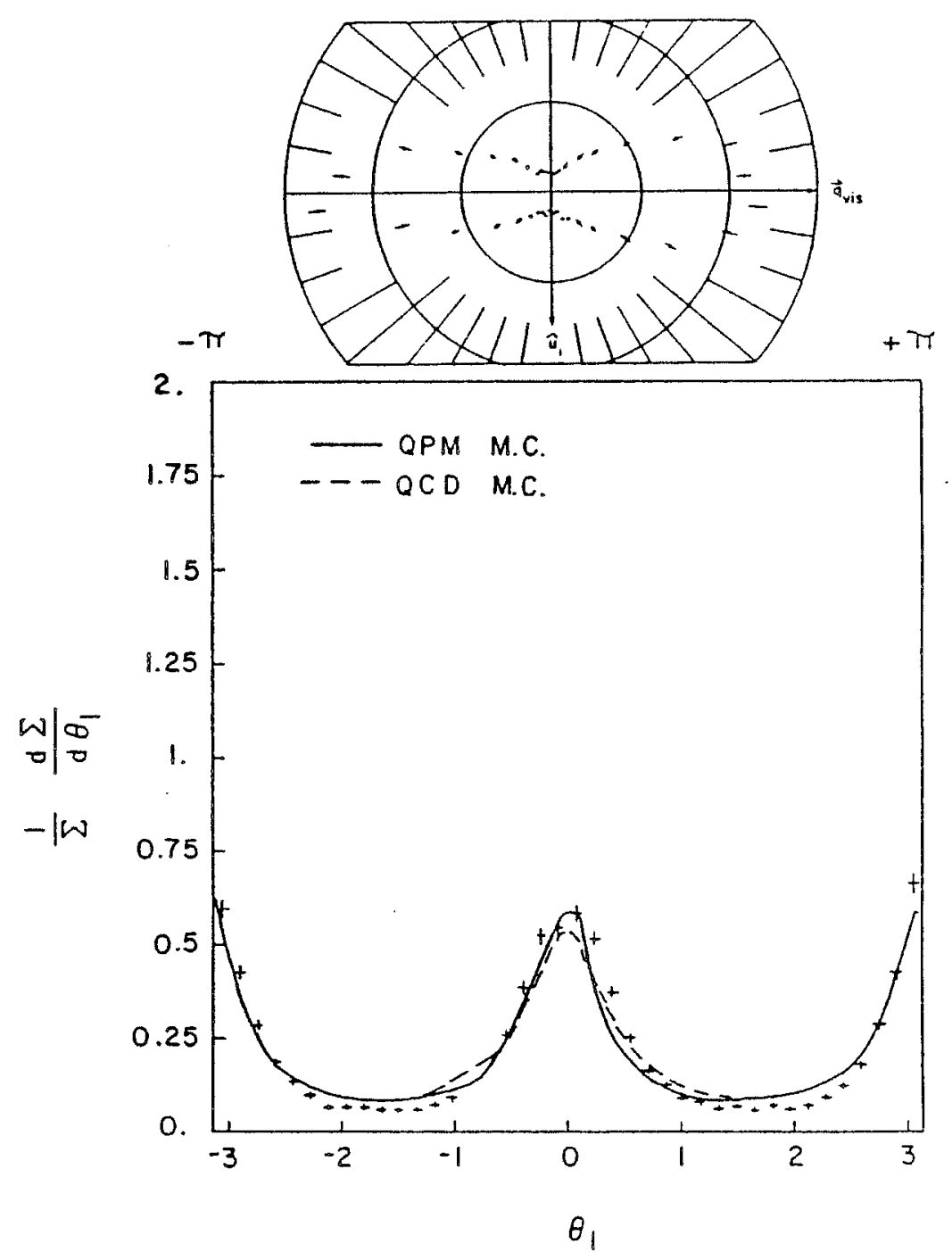

Figure $V-37(a)$ 

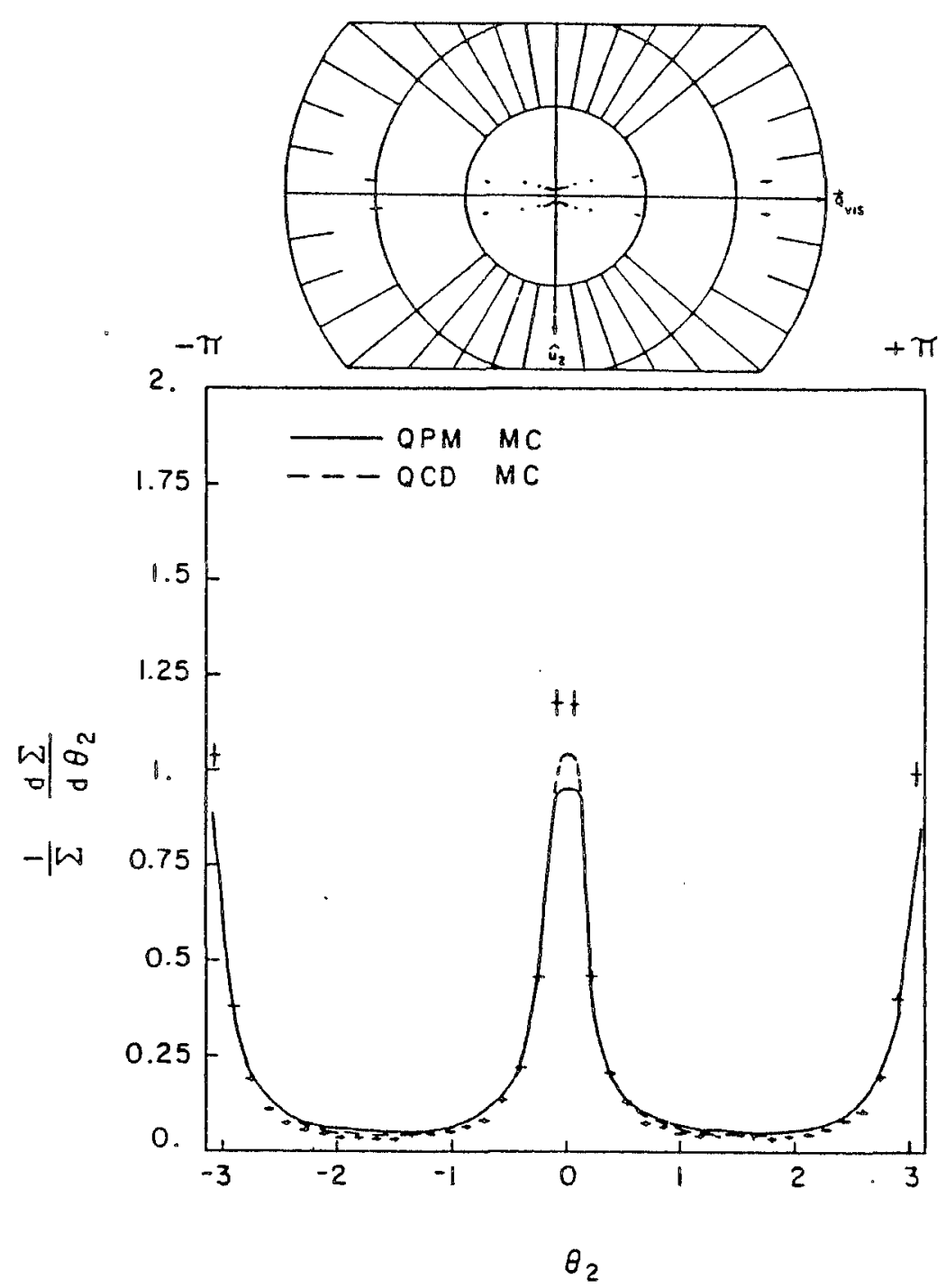

Figure $V-31(b)$

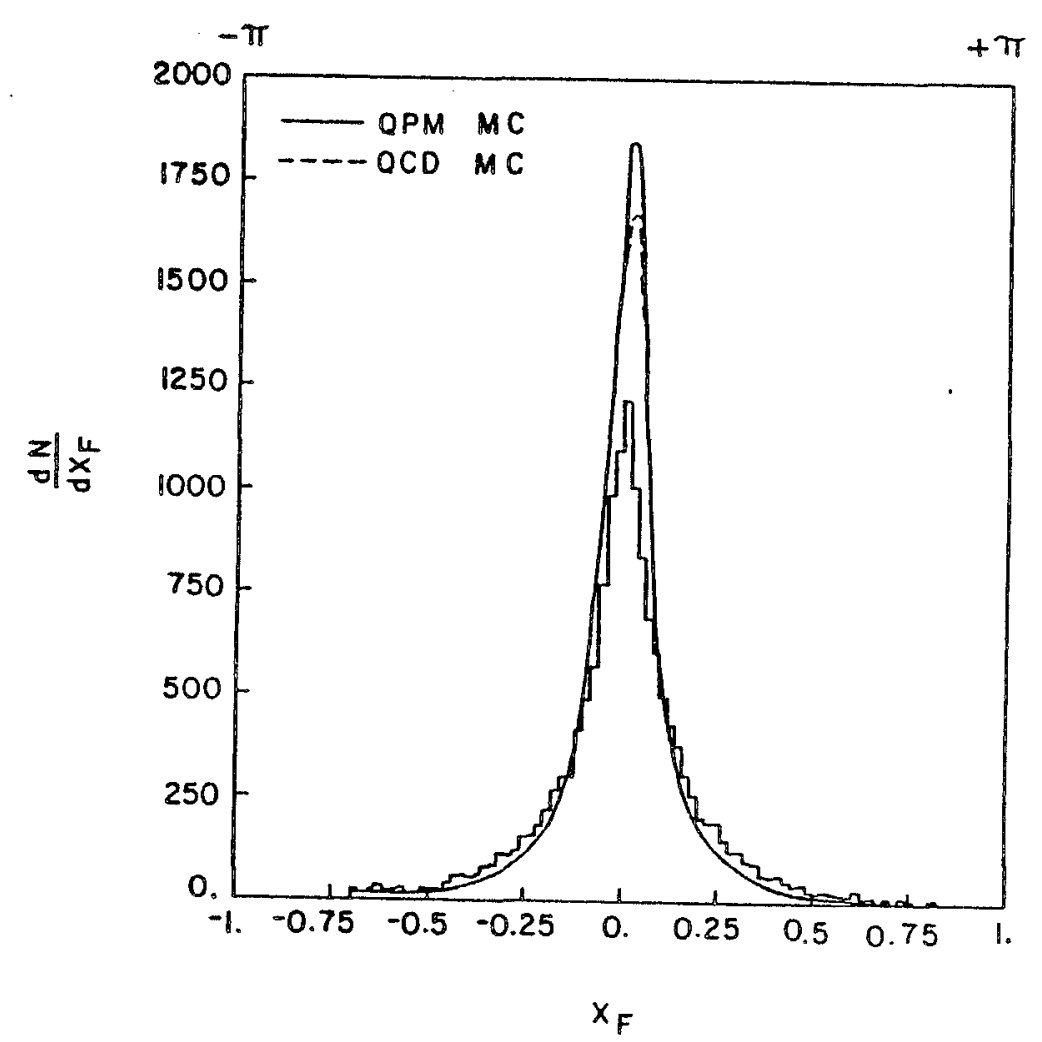

Figure $V-32$ 


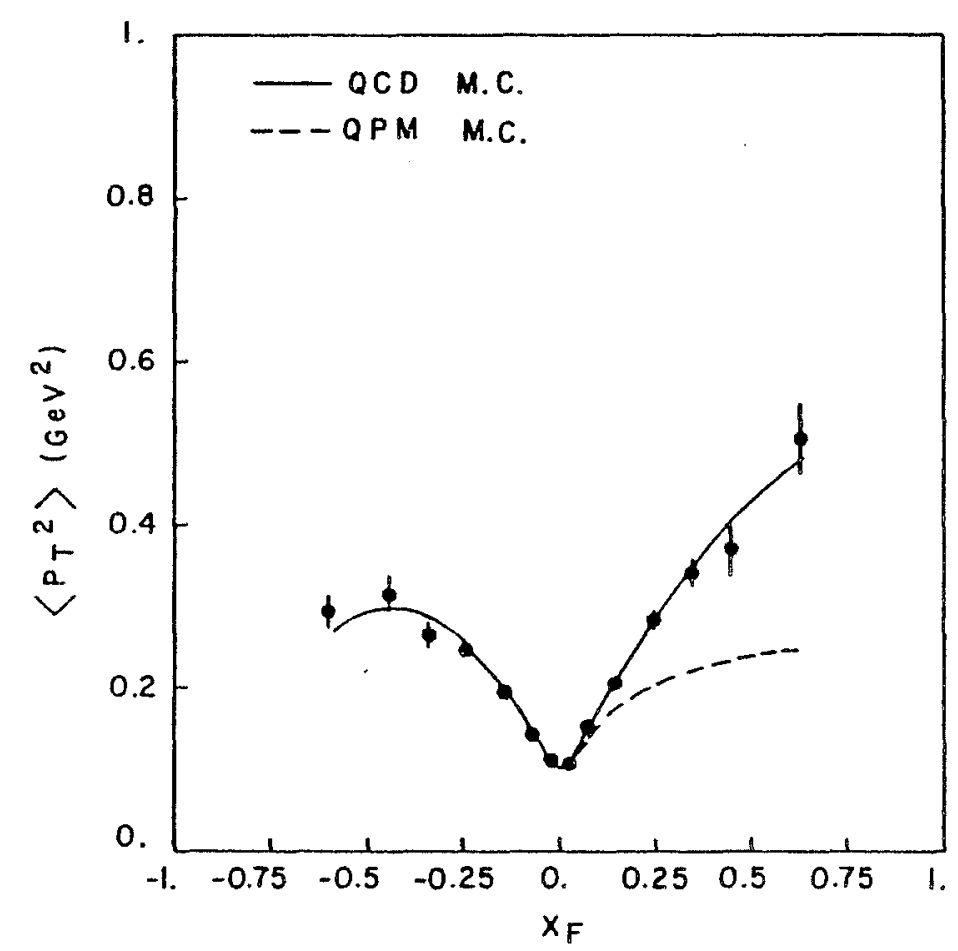

Figure $V-33$

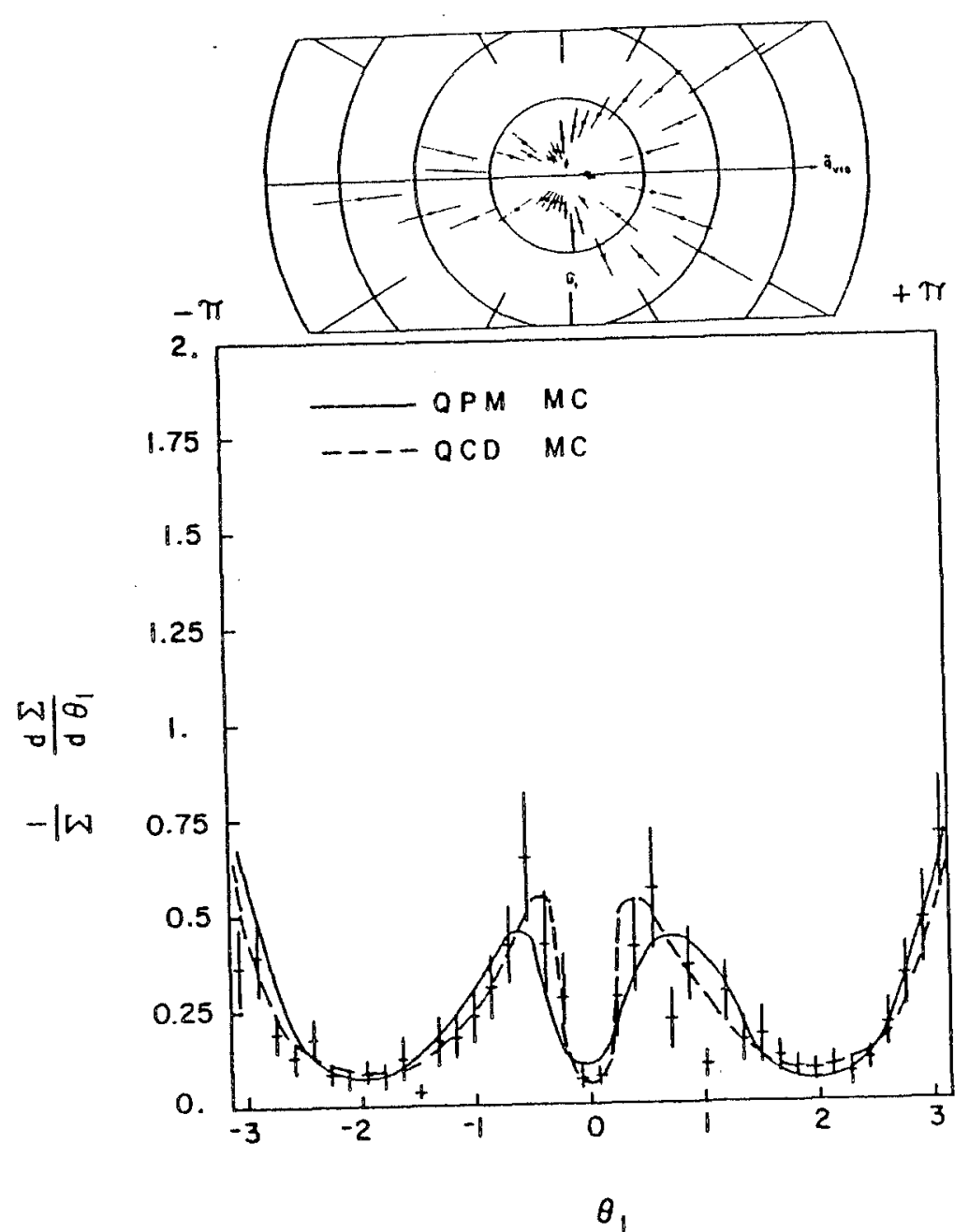

Eigure V-34(a) 

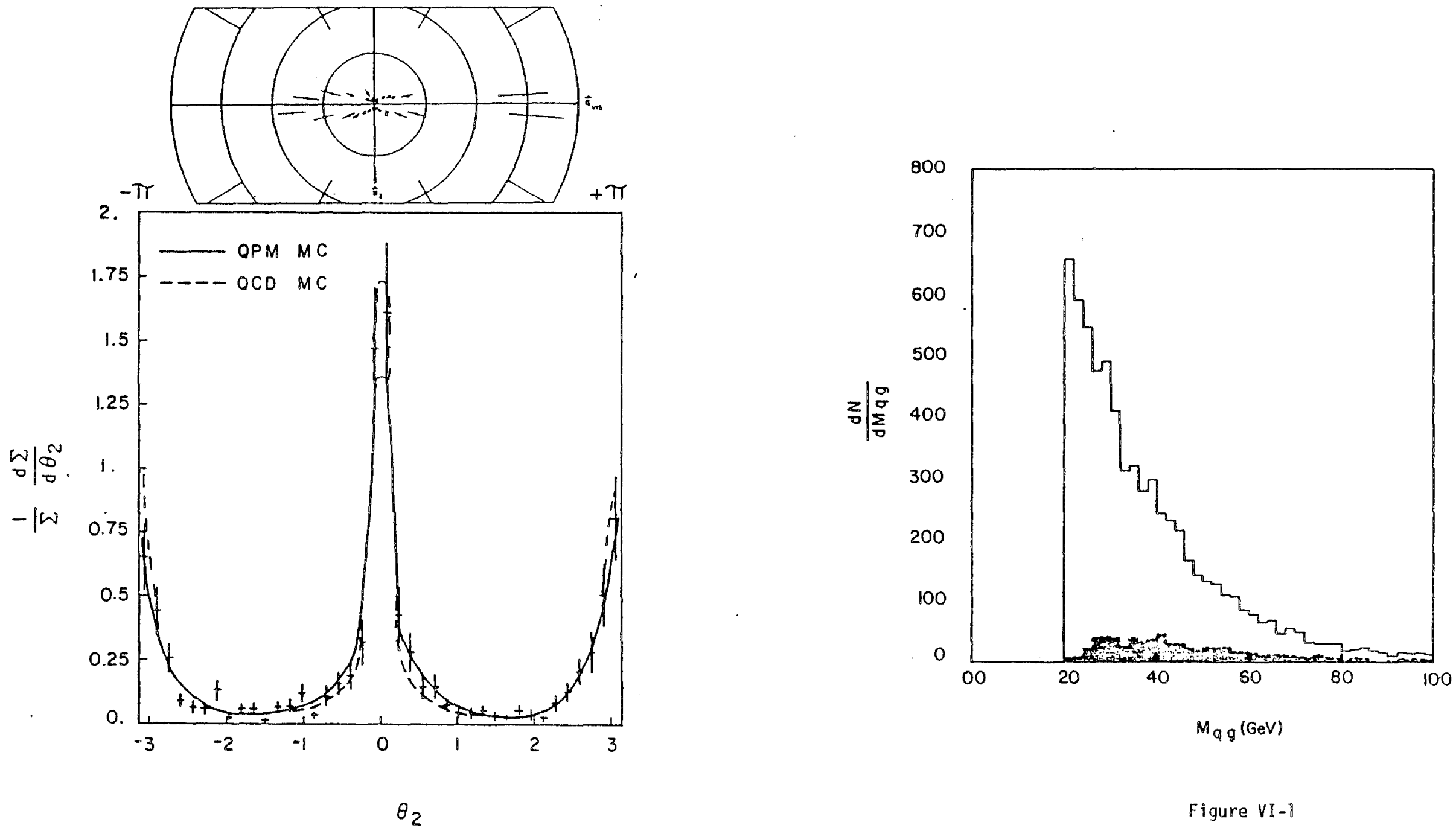

Figure V-34(b) 


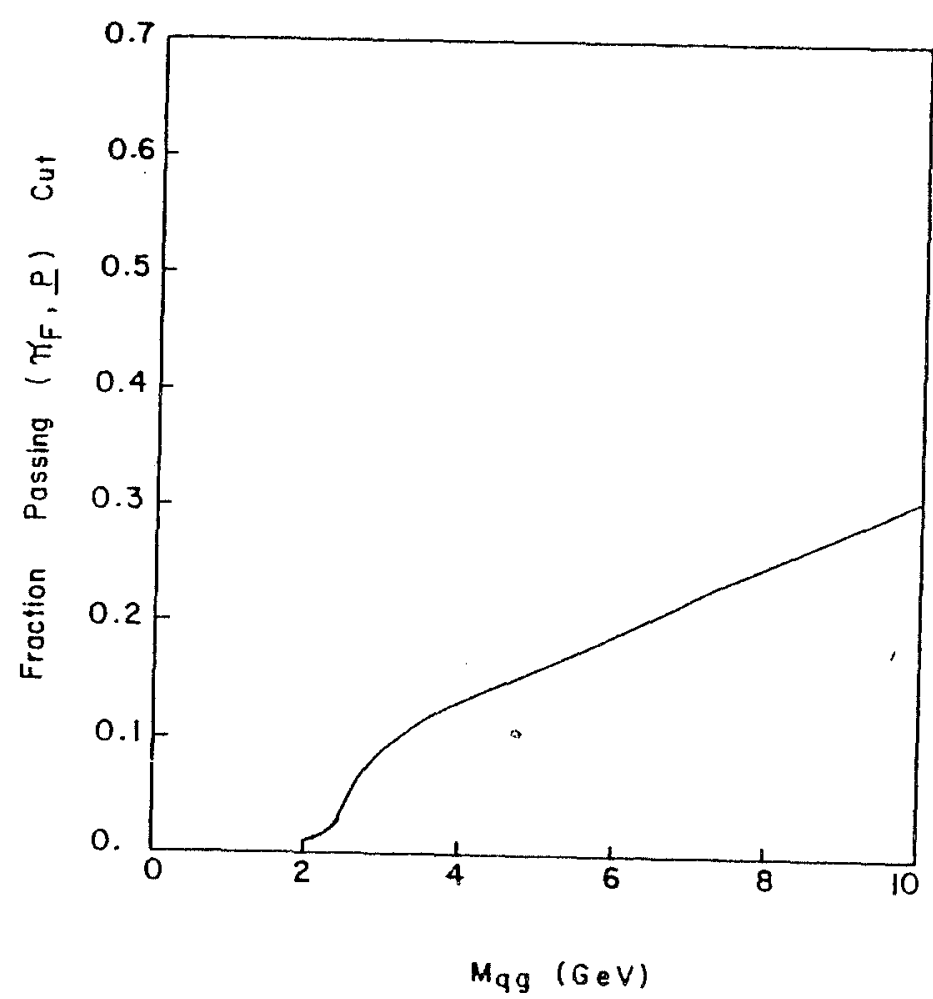

Figure VI-2

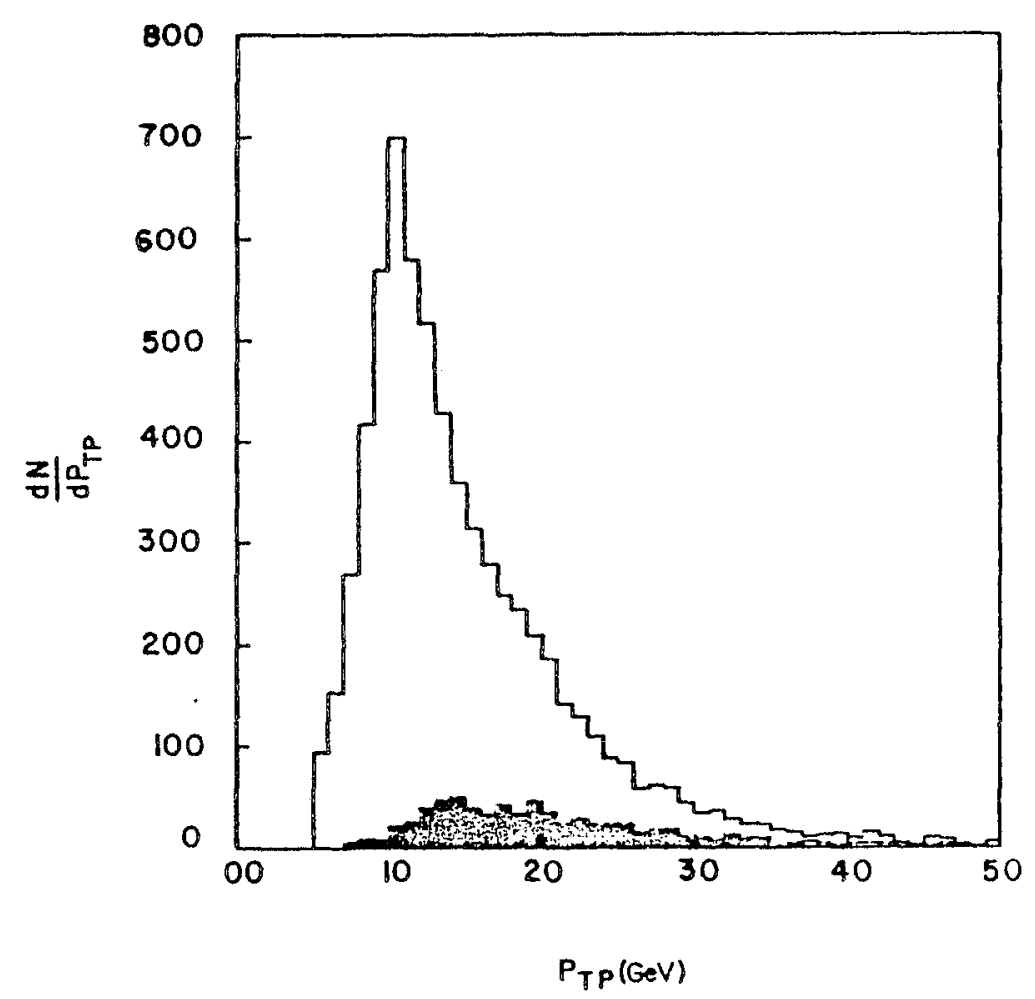

Figure VI-3 


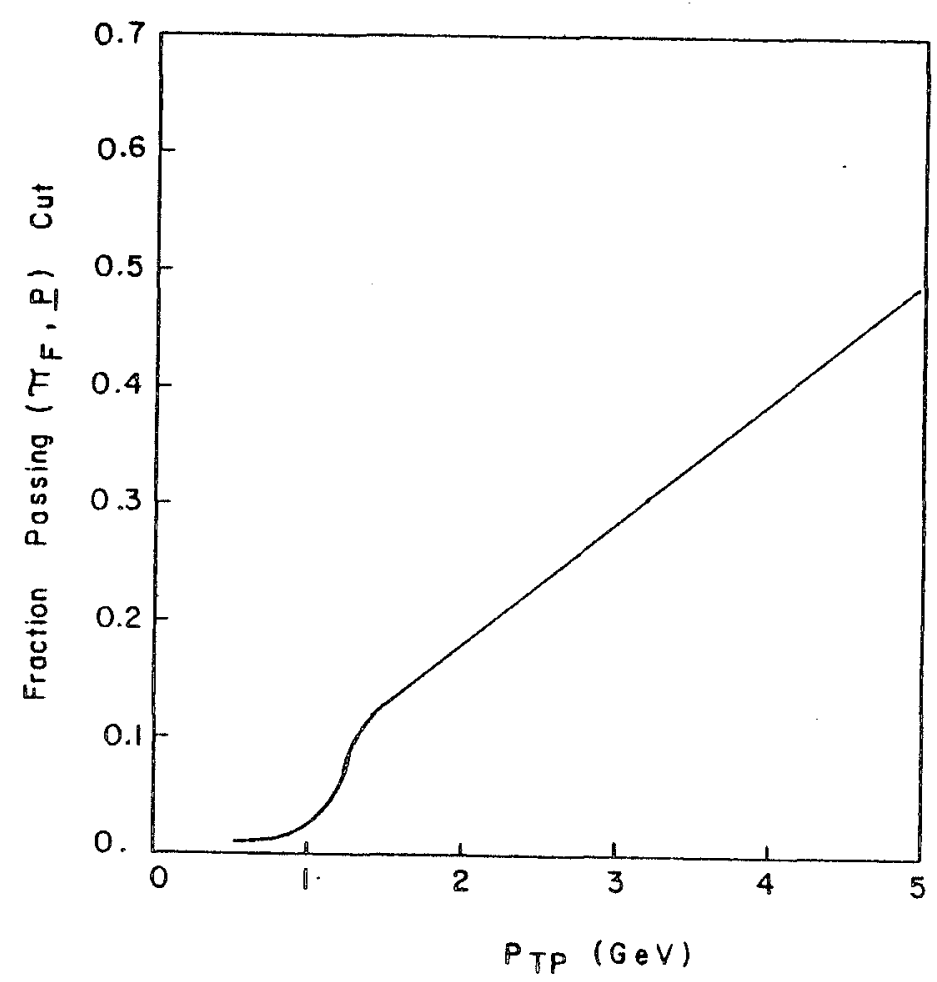

Figure IV-4

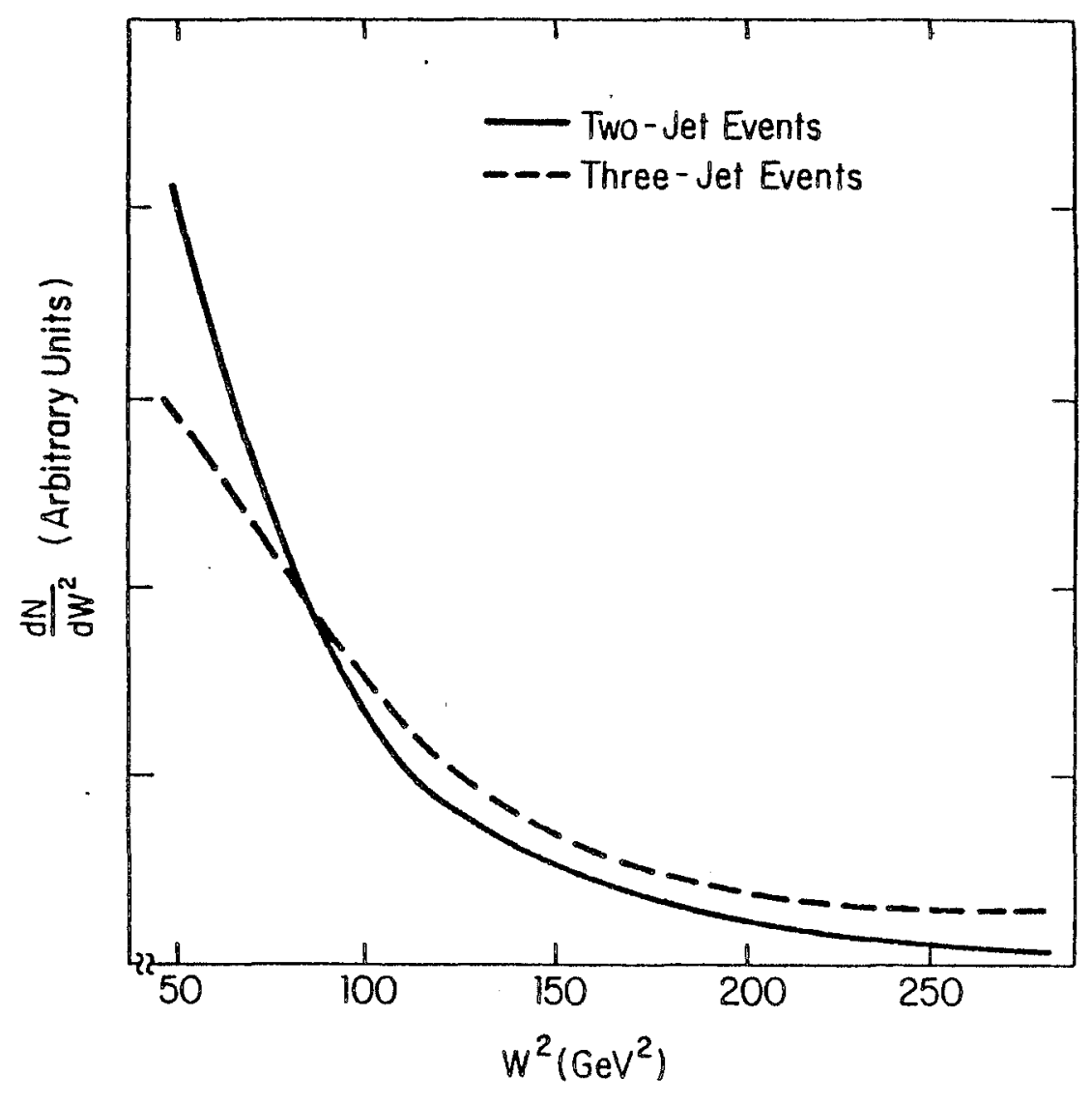

Figure VI-5 


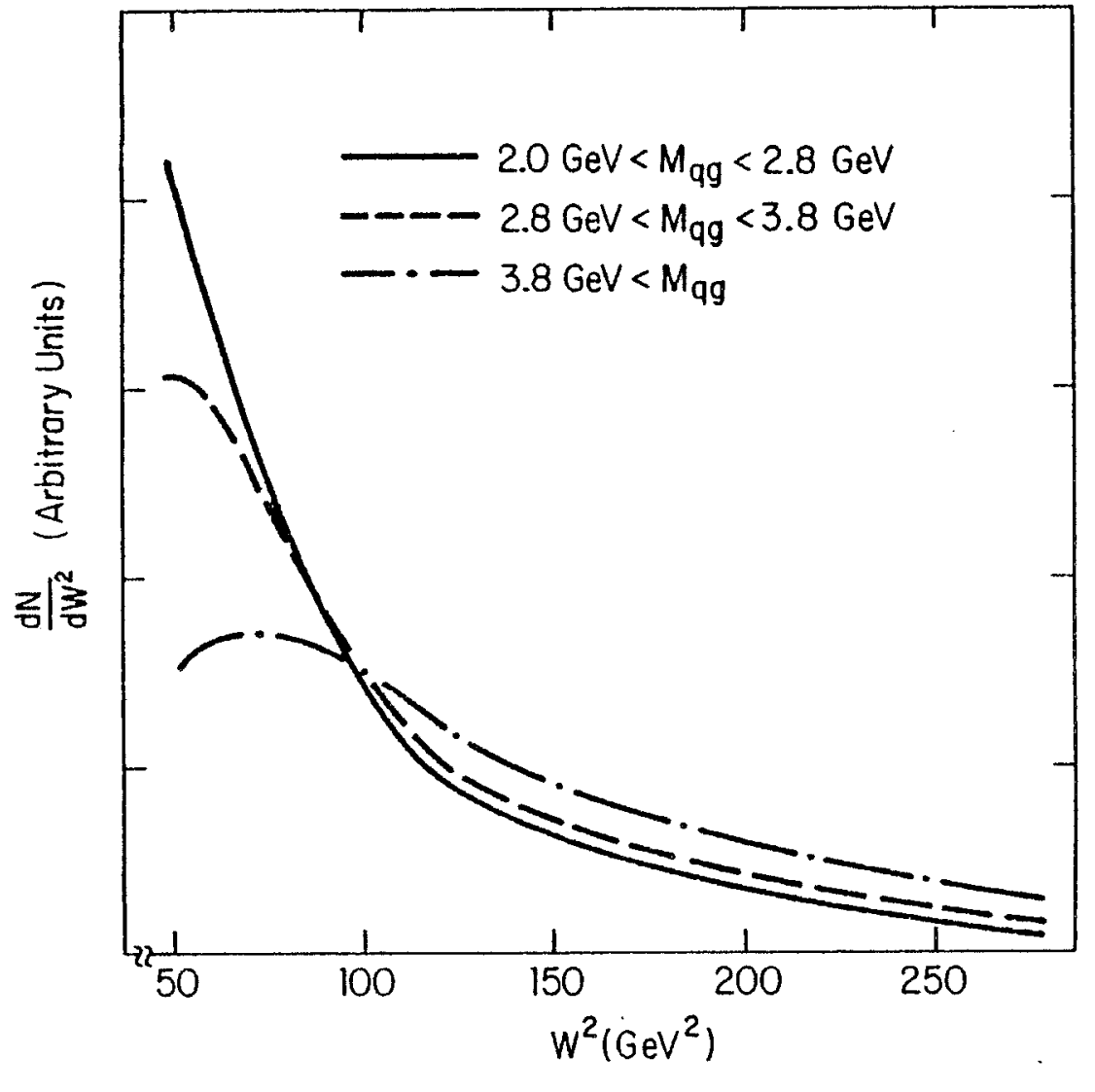

Figure VI-6(a)

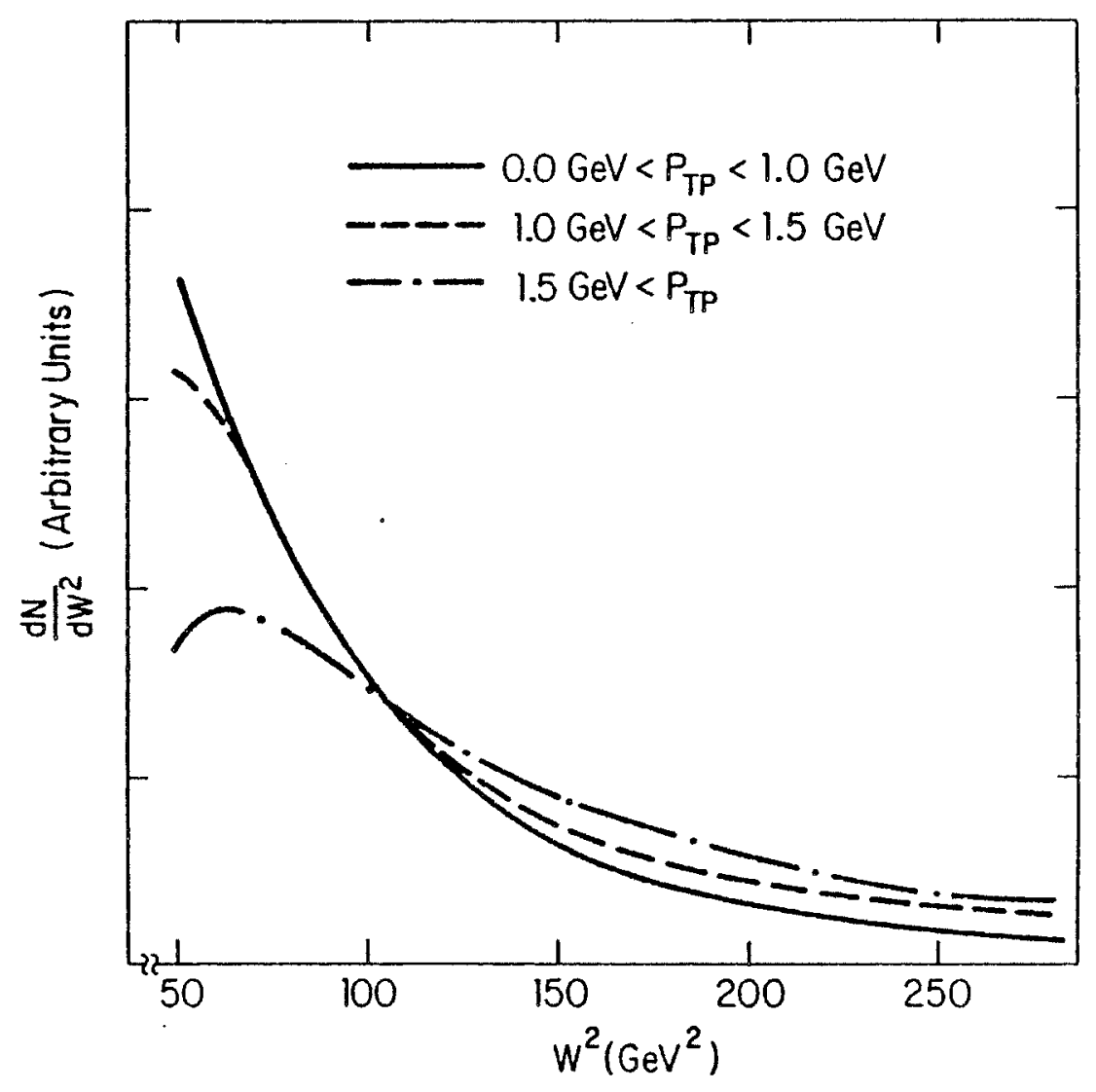

Figure VI-6(b) 


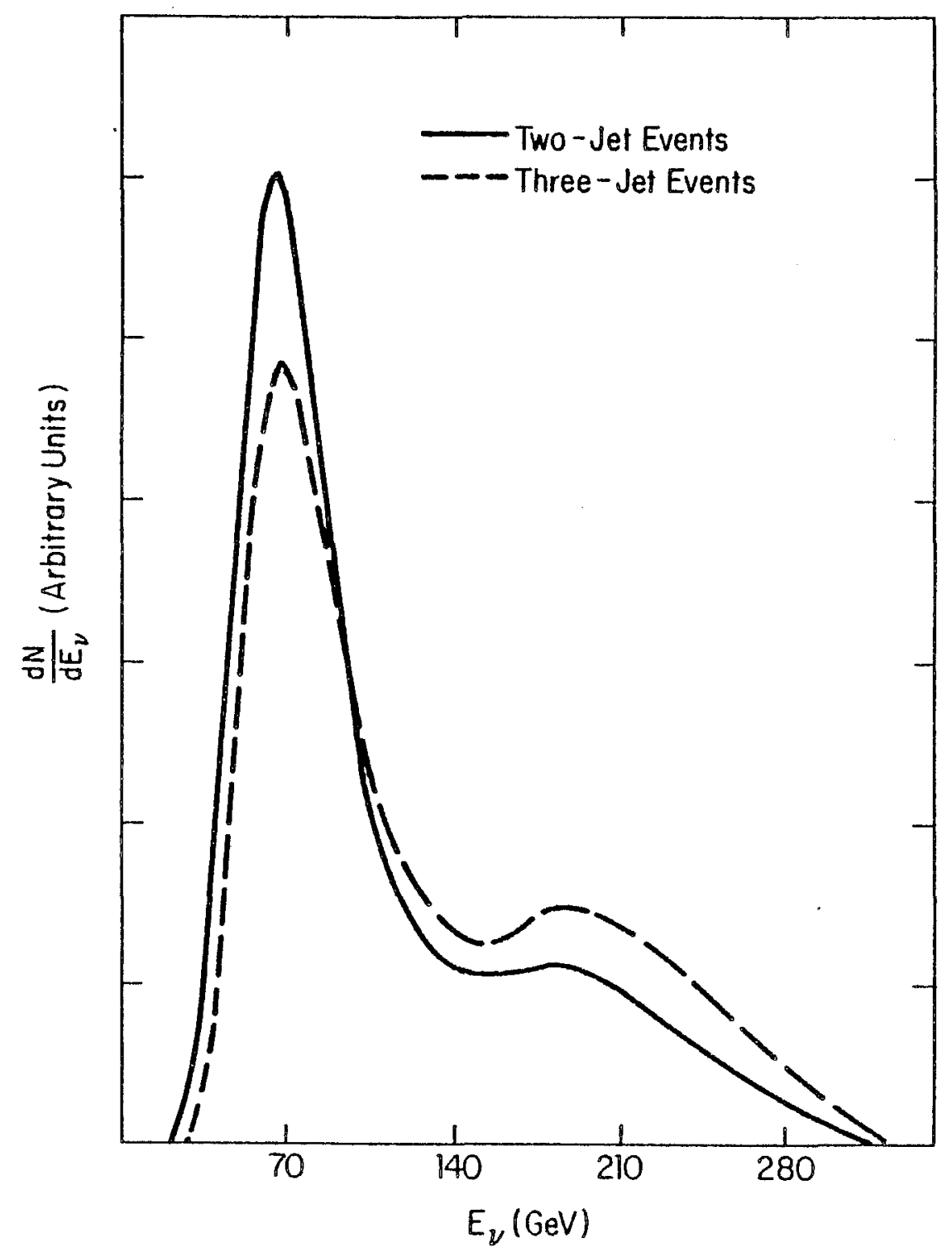

Figure VI-7

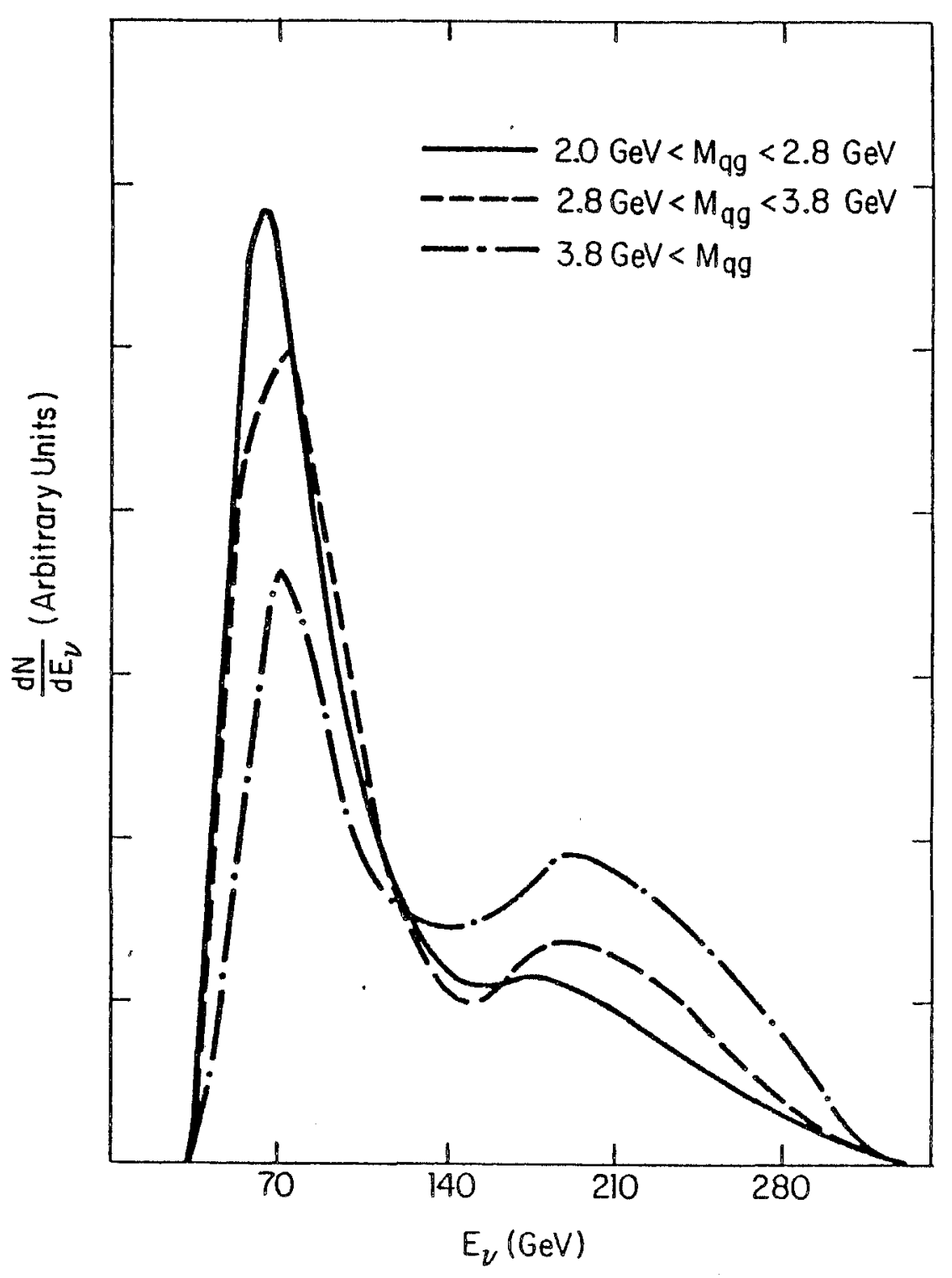

Figure VI-8(a) 


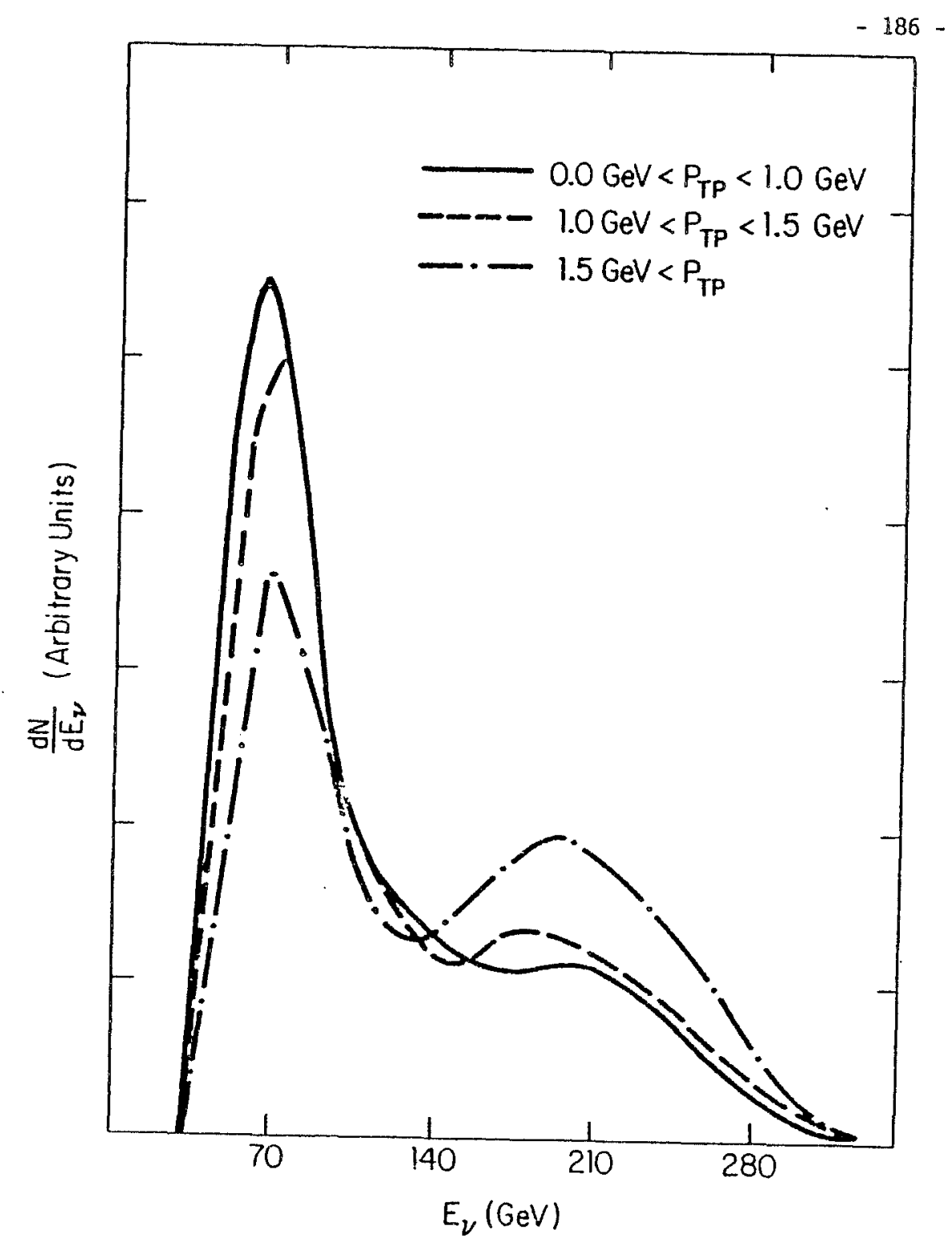

Figure VI-8(b)

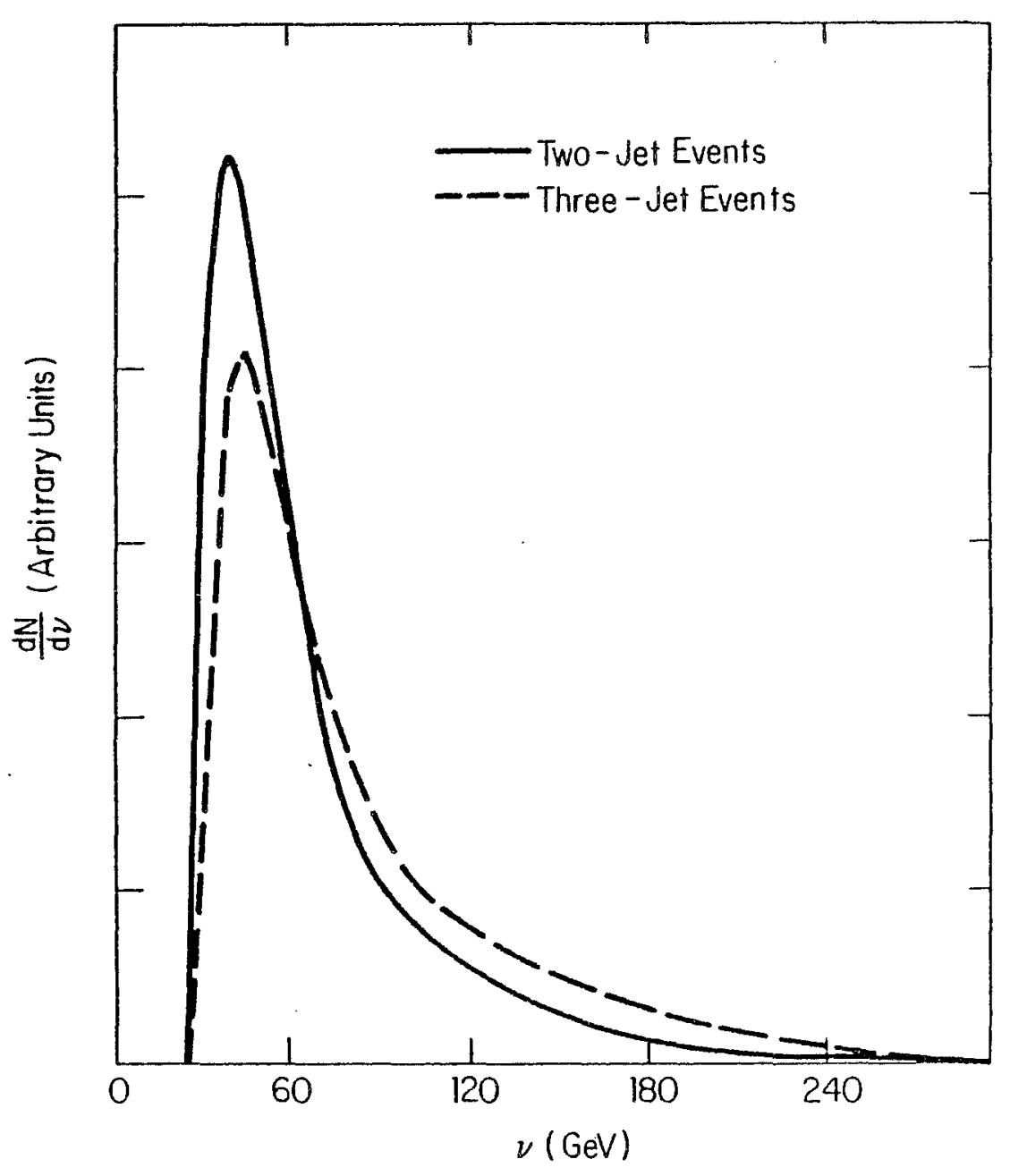

Figure VI-9 


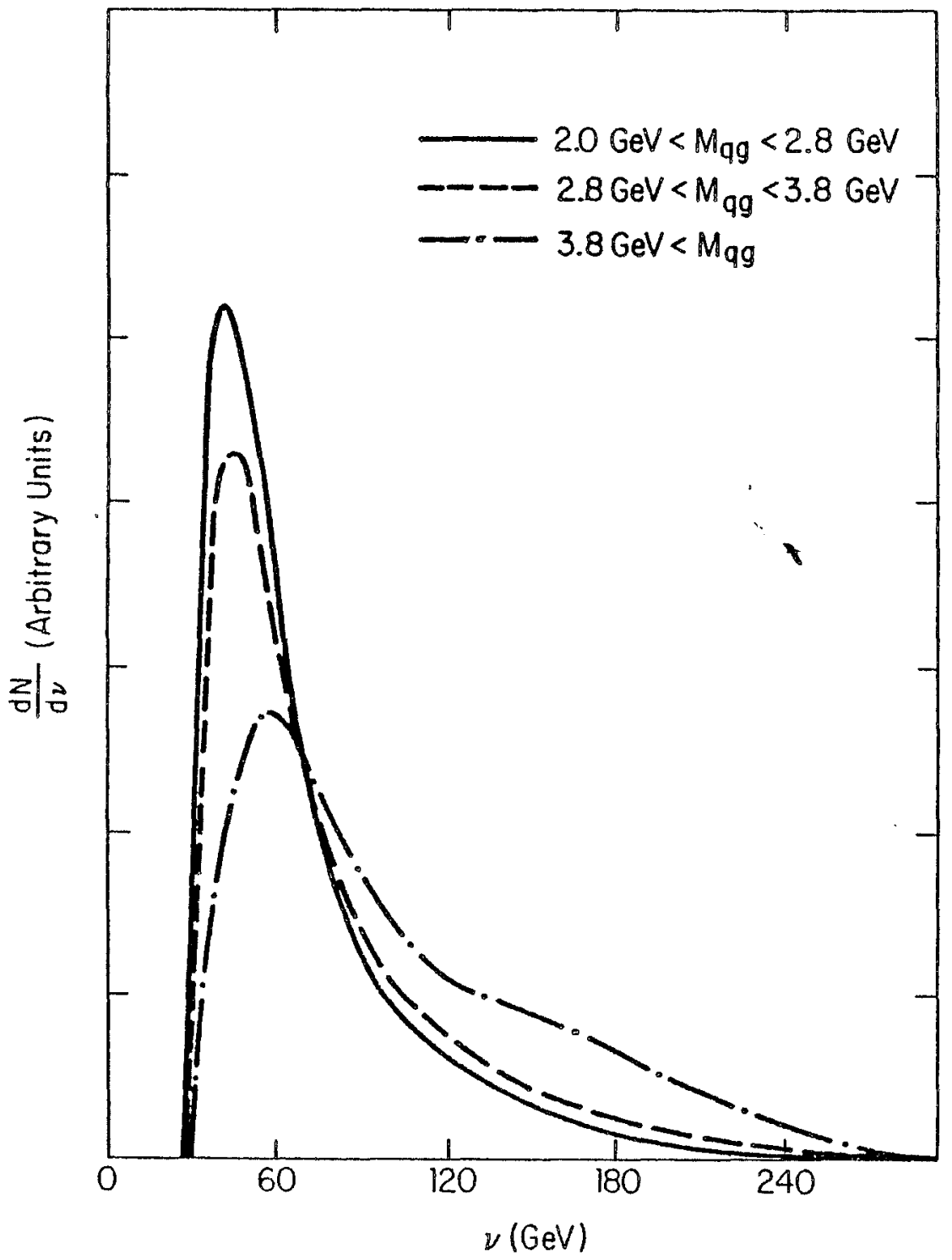

Figure VI-10(a)

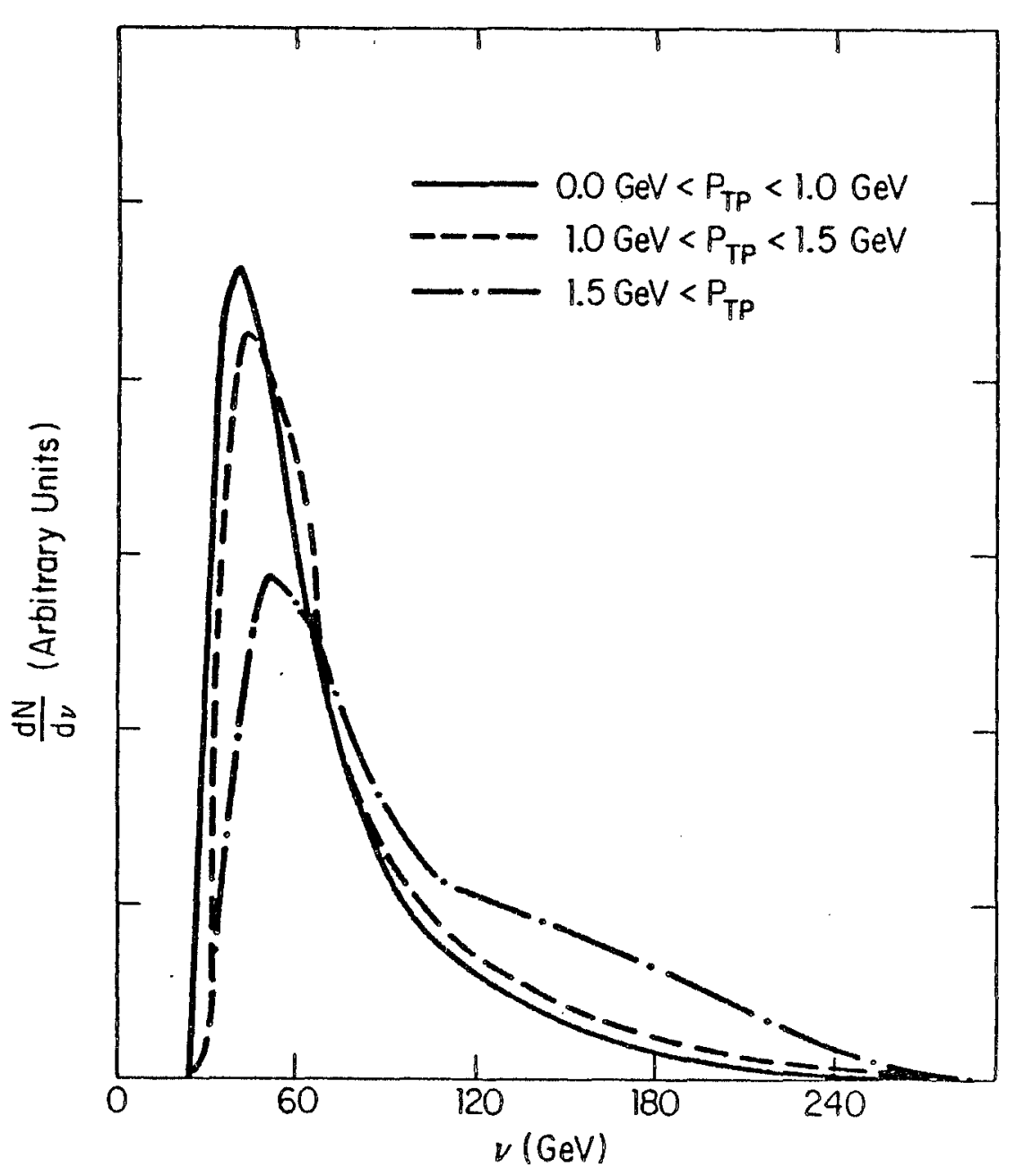

Figure VI-10(b) 


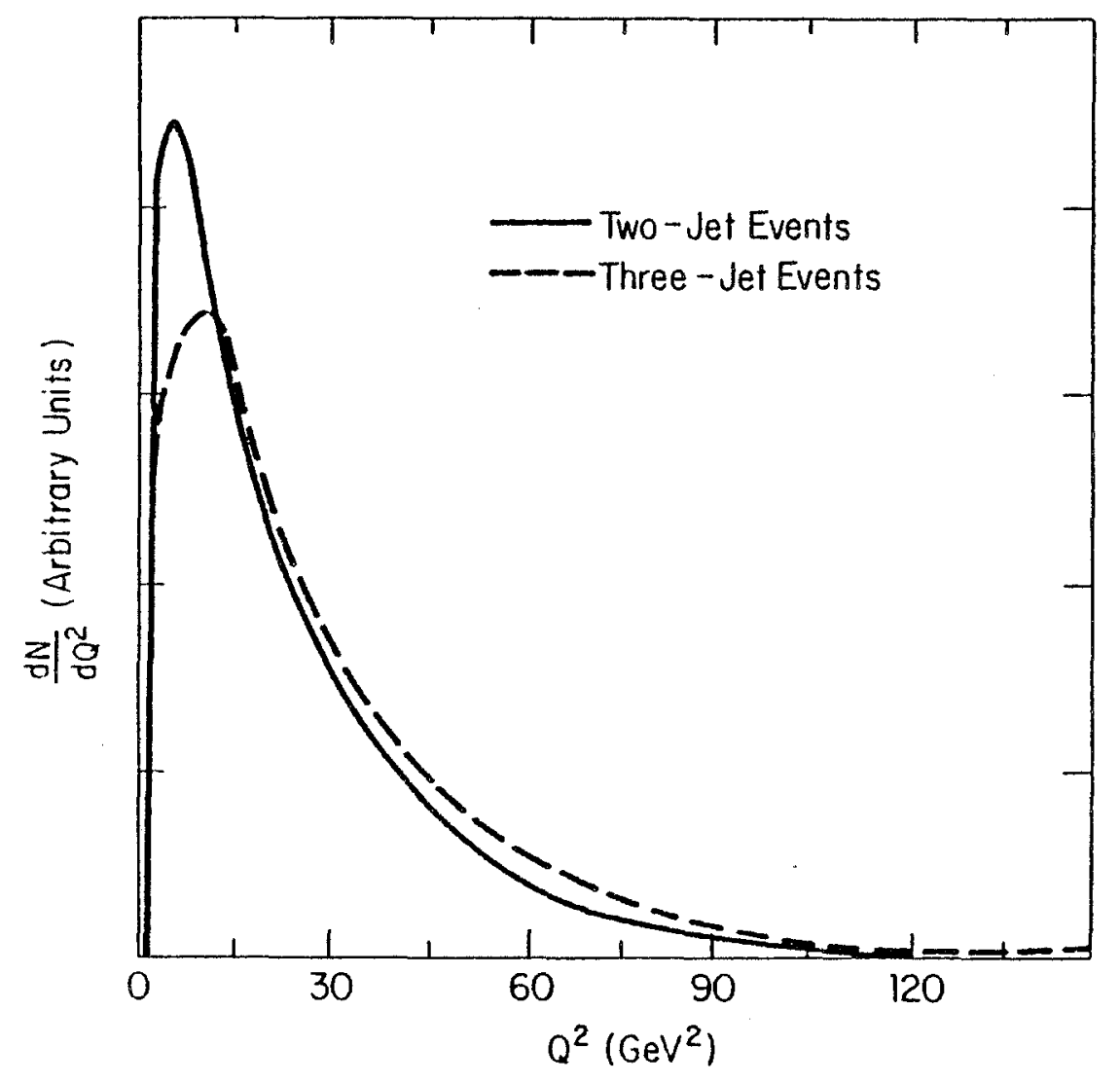

Figure VI-11

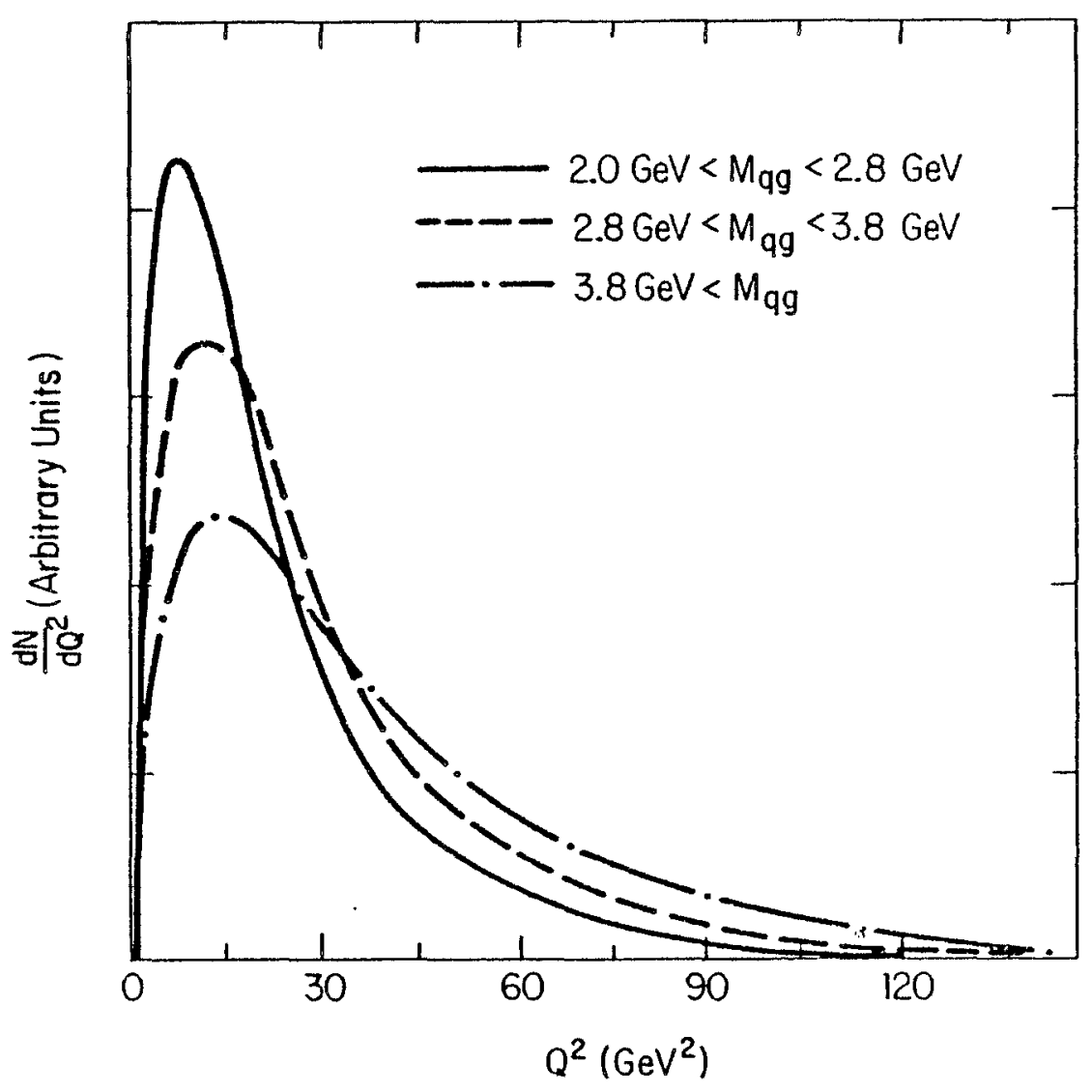

Figure VI-12(a) 


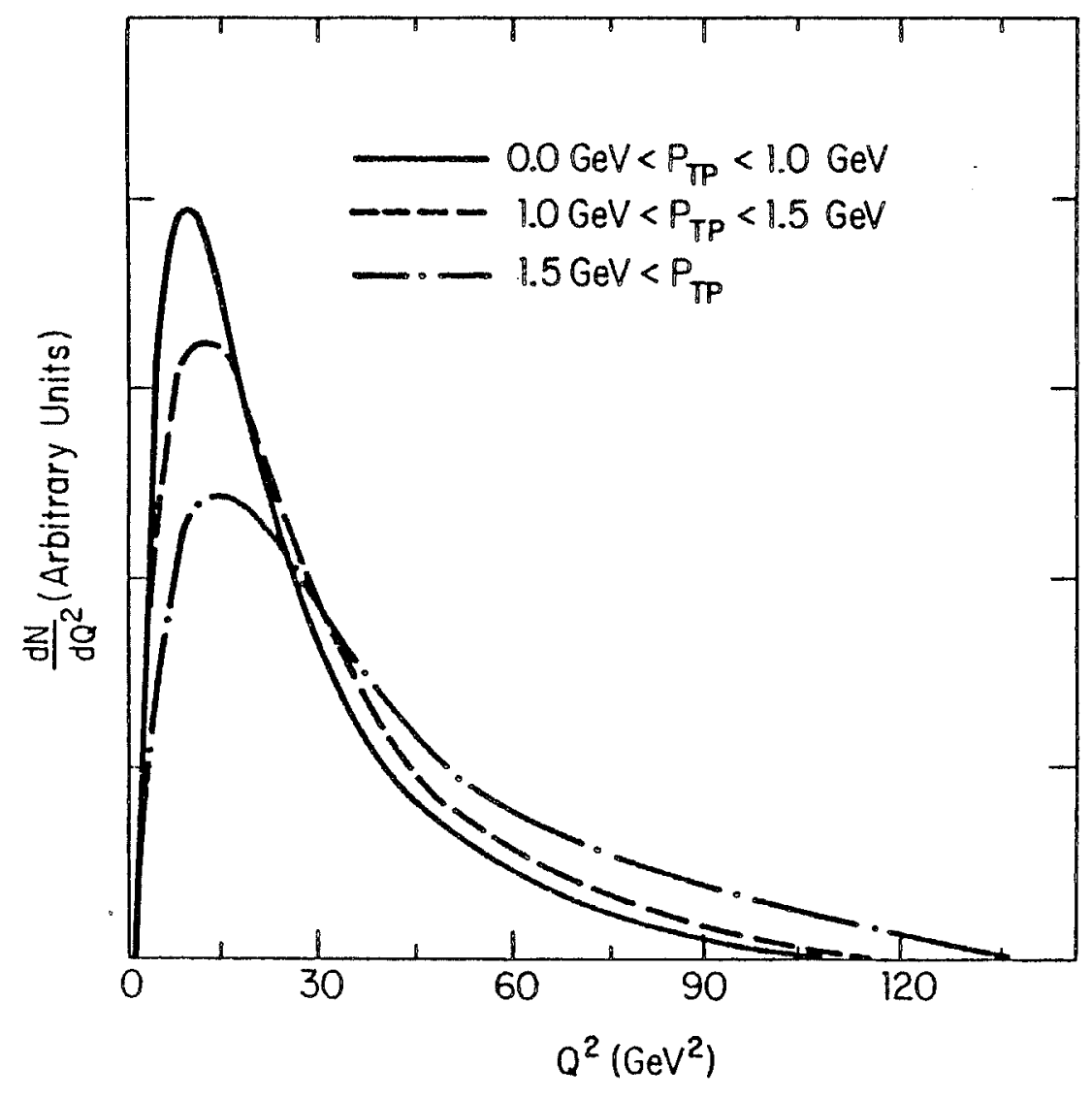

Figure VI-12(b)

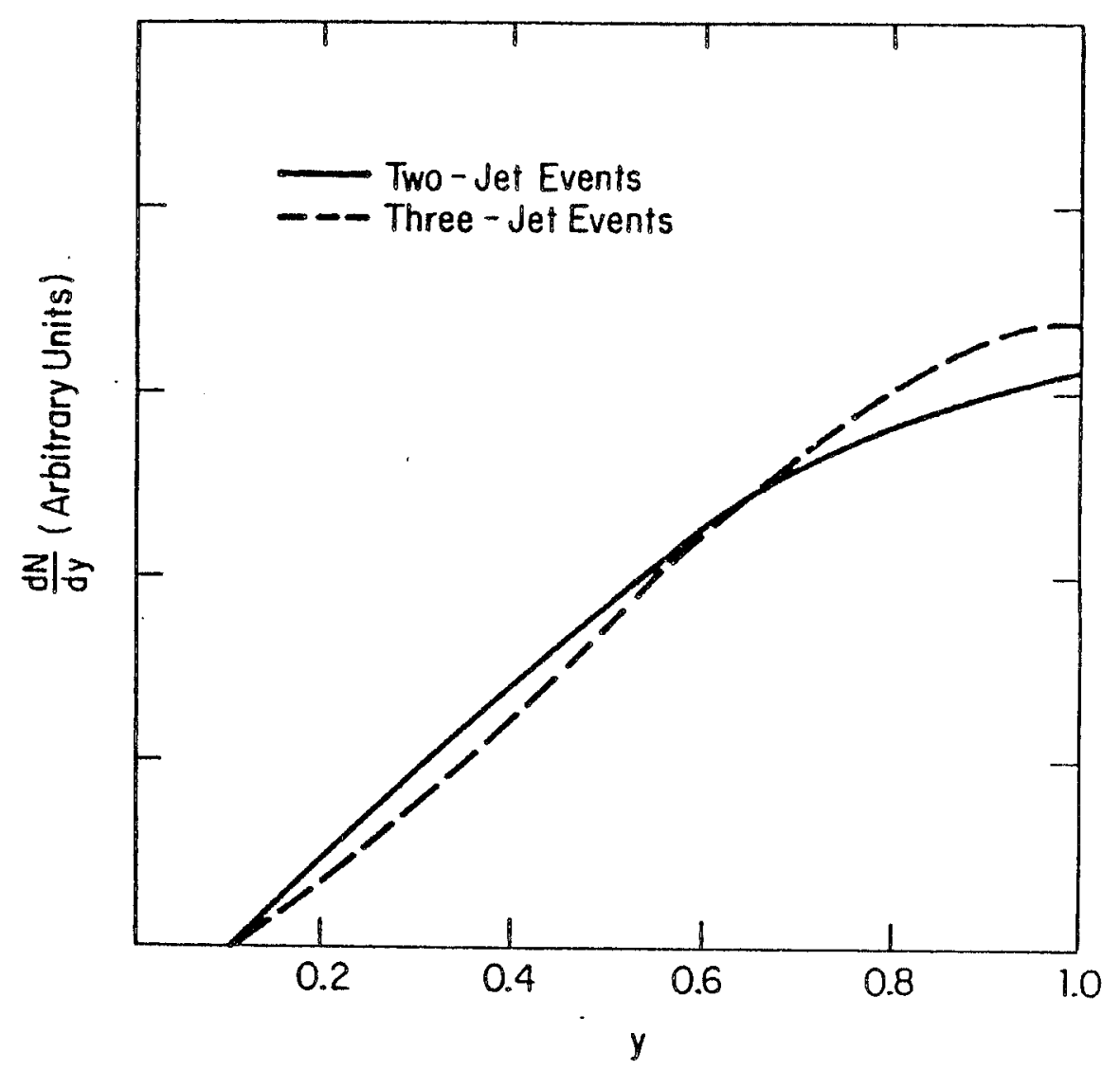

Figure VI-13 


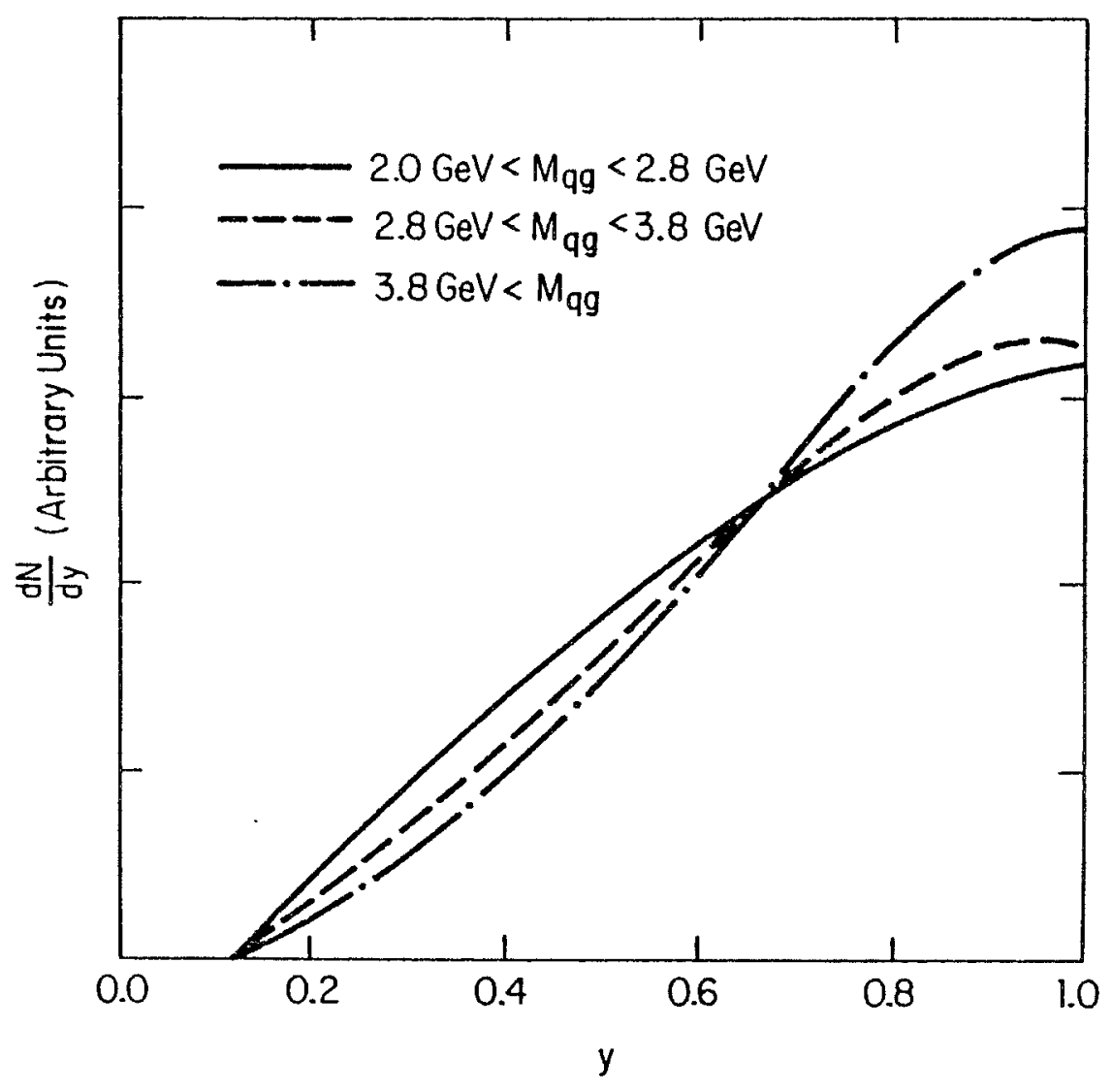

Figure VI-14(a)

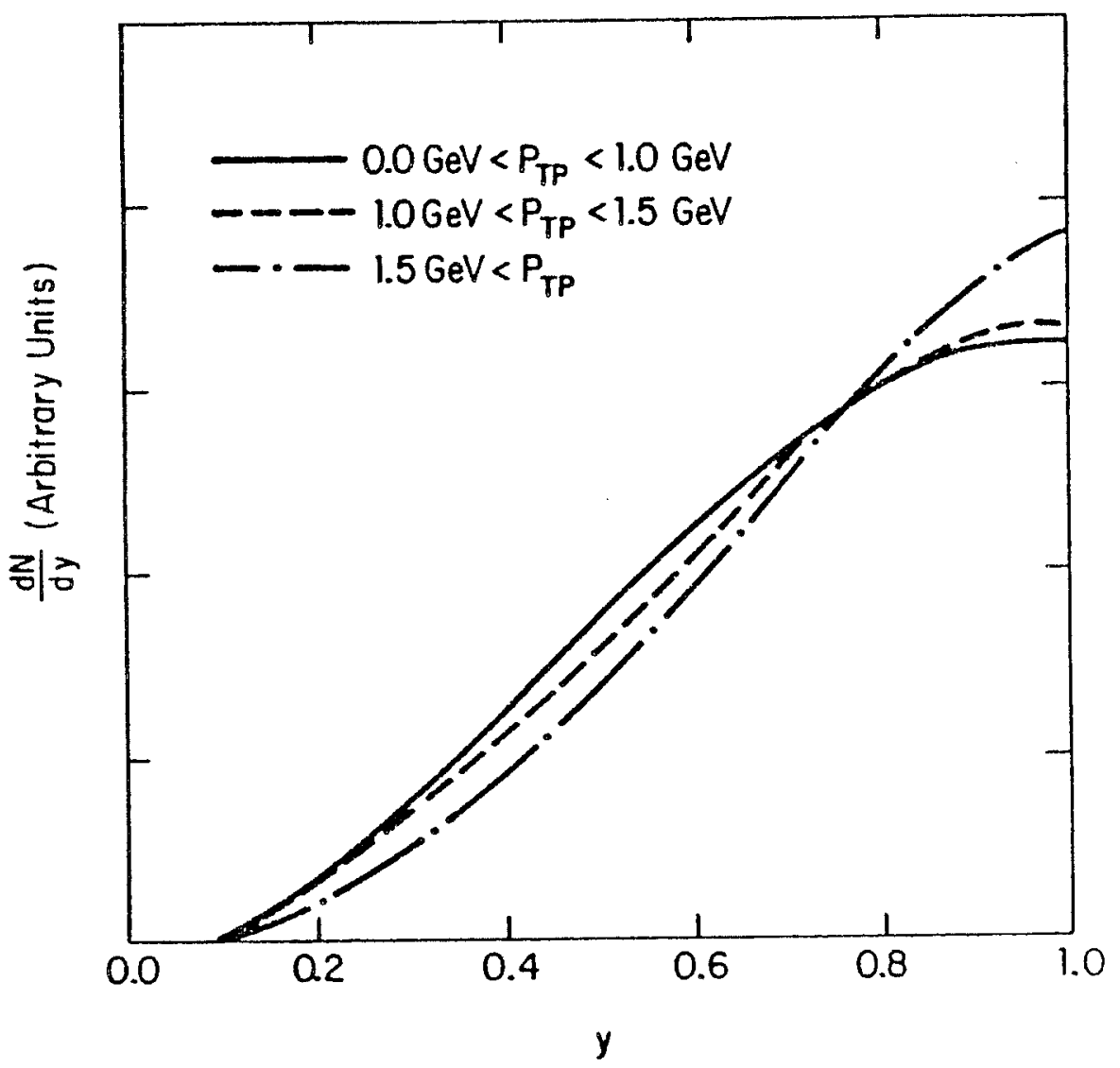

Figure VI-14(b) 

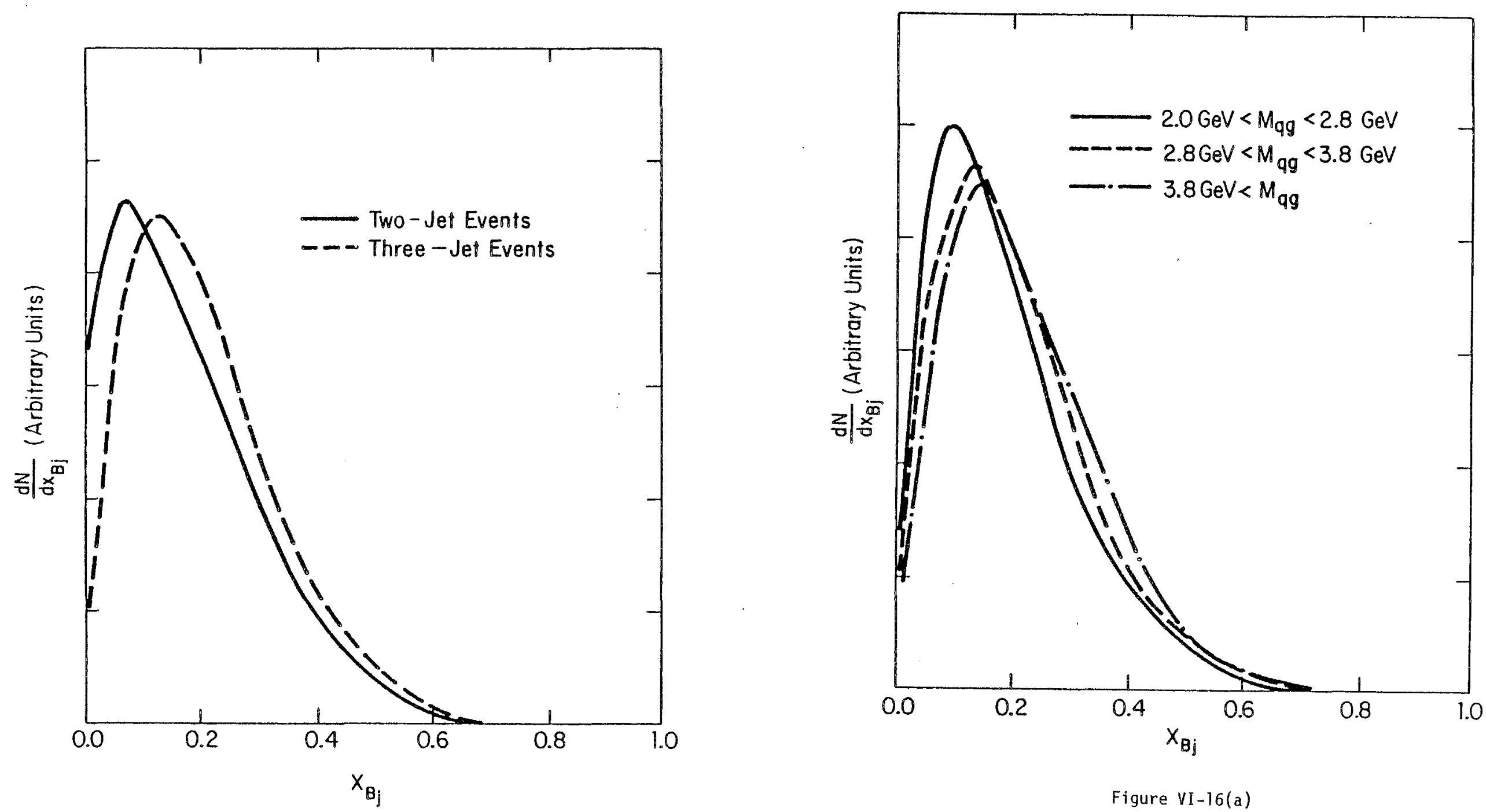

Figure VI-15 


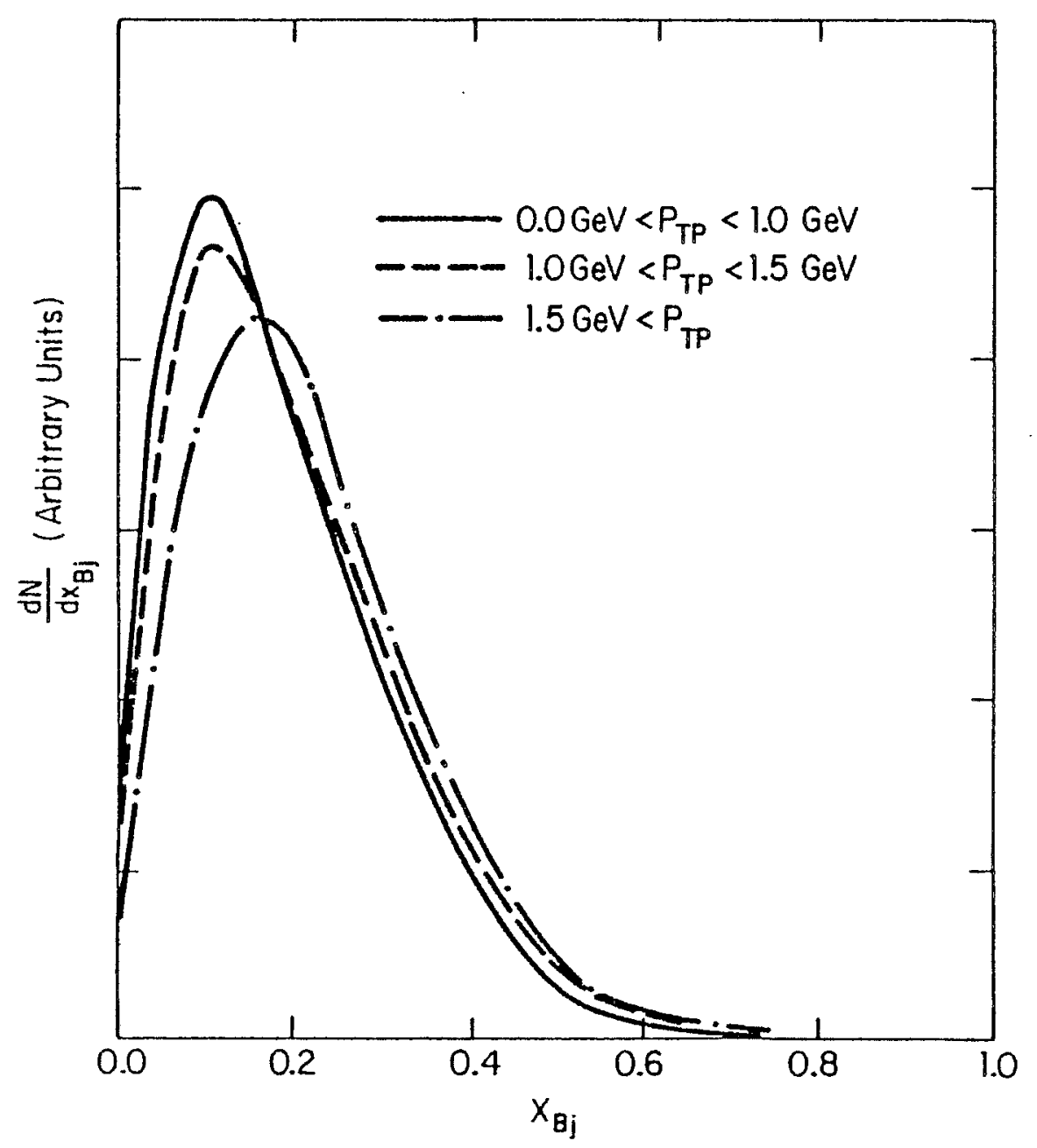

Figure VI-16(b)

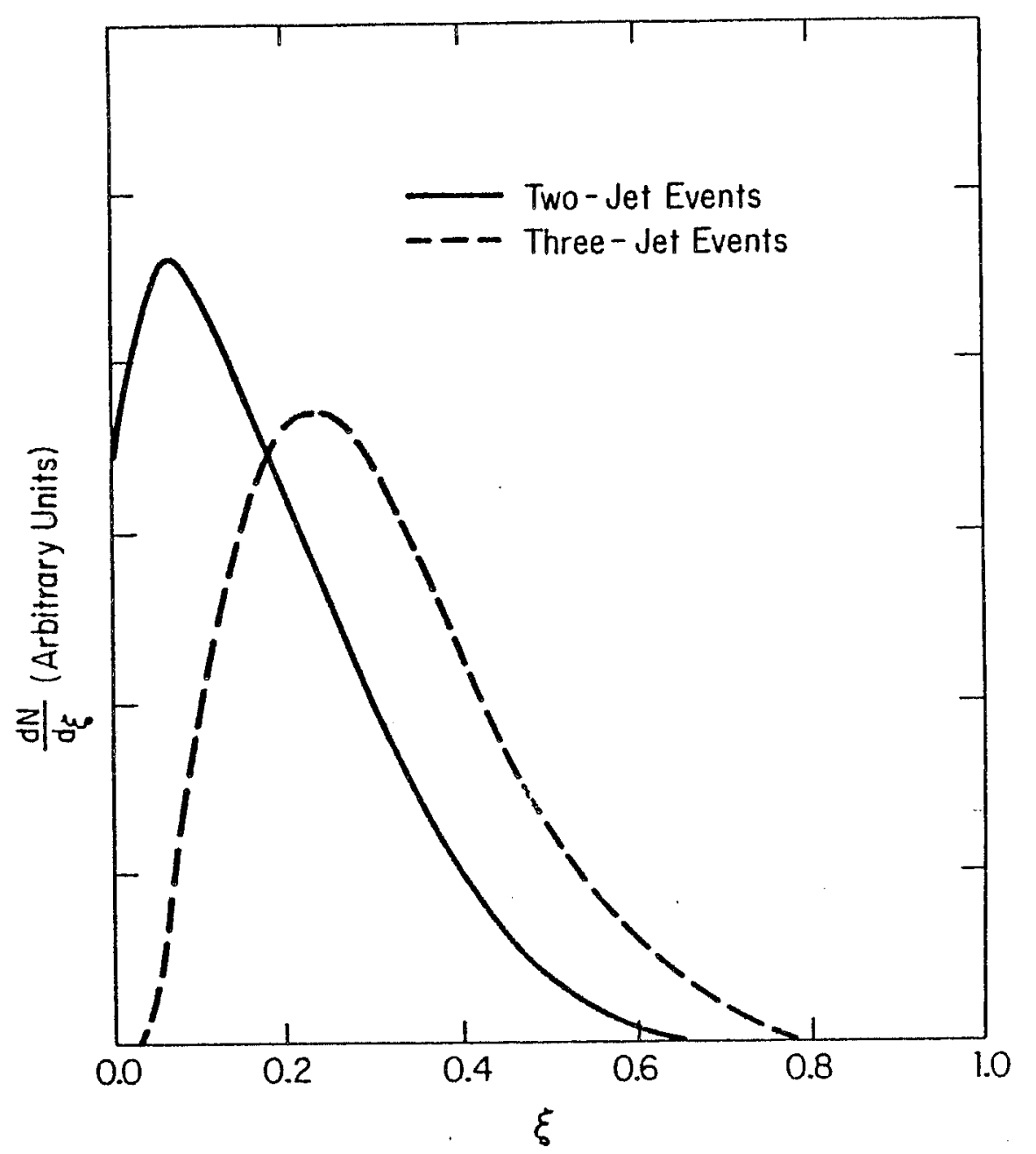

Figure IV-17 


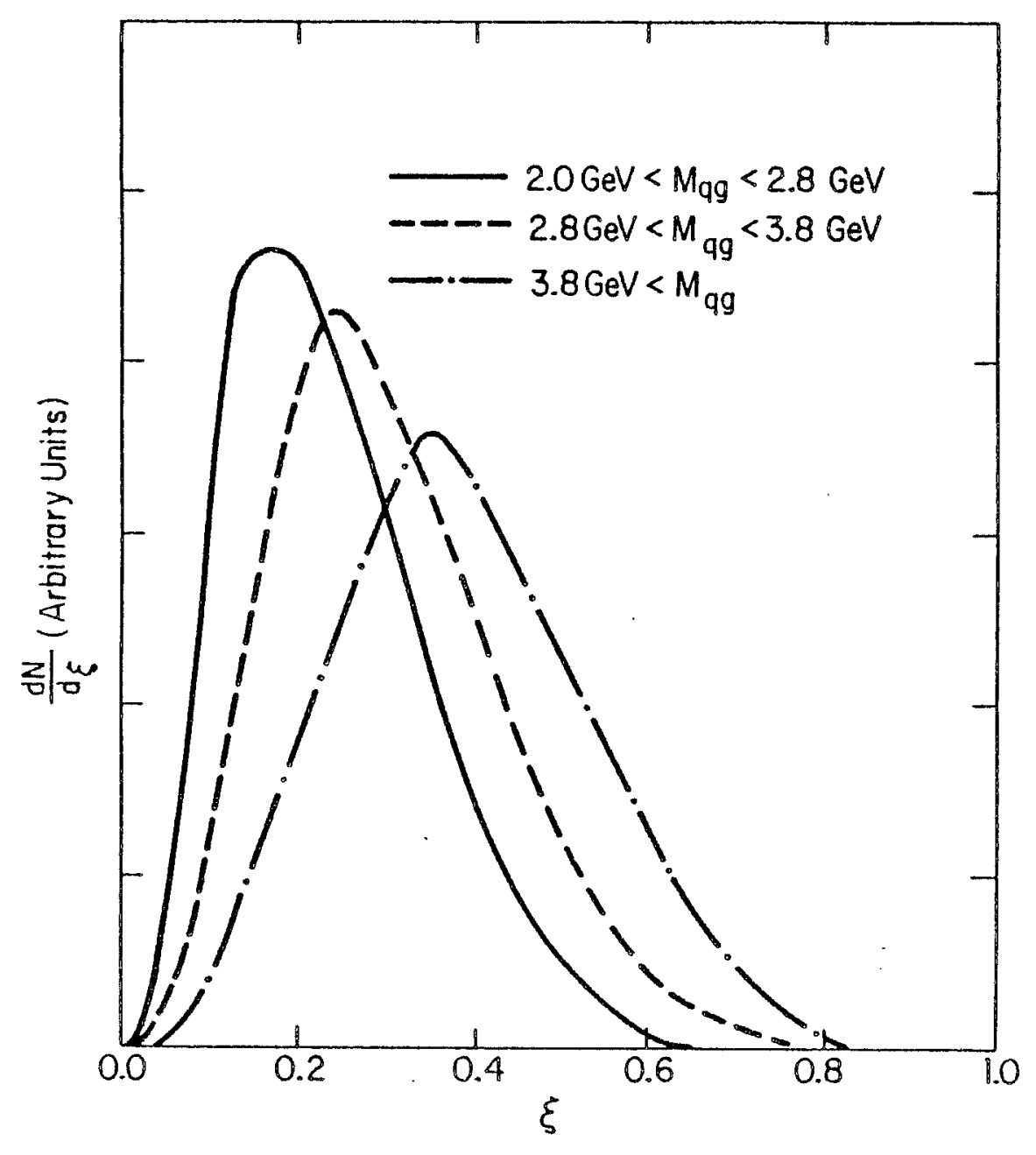

Figure VI-18(a)

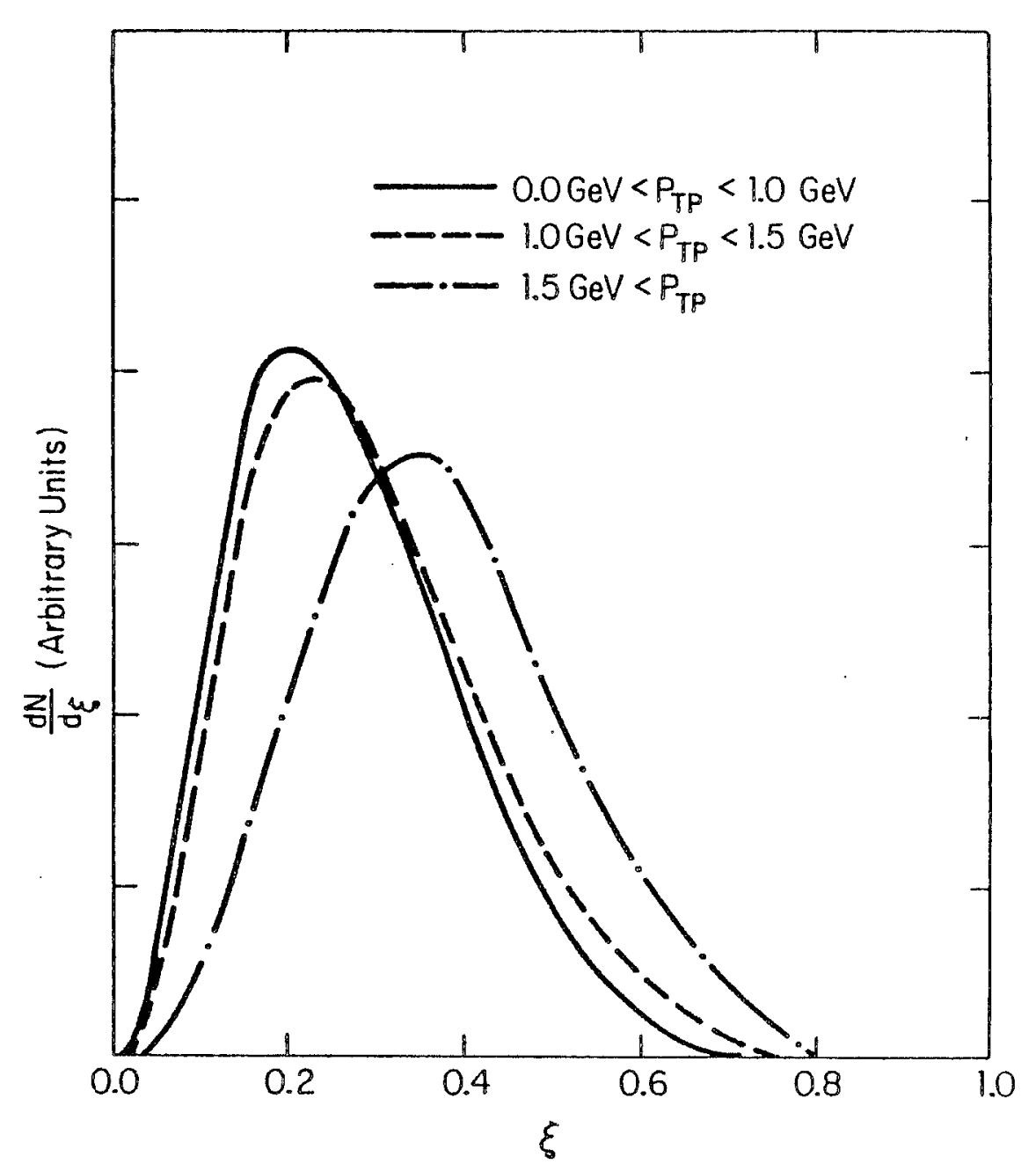

Figure VI-18(b) 


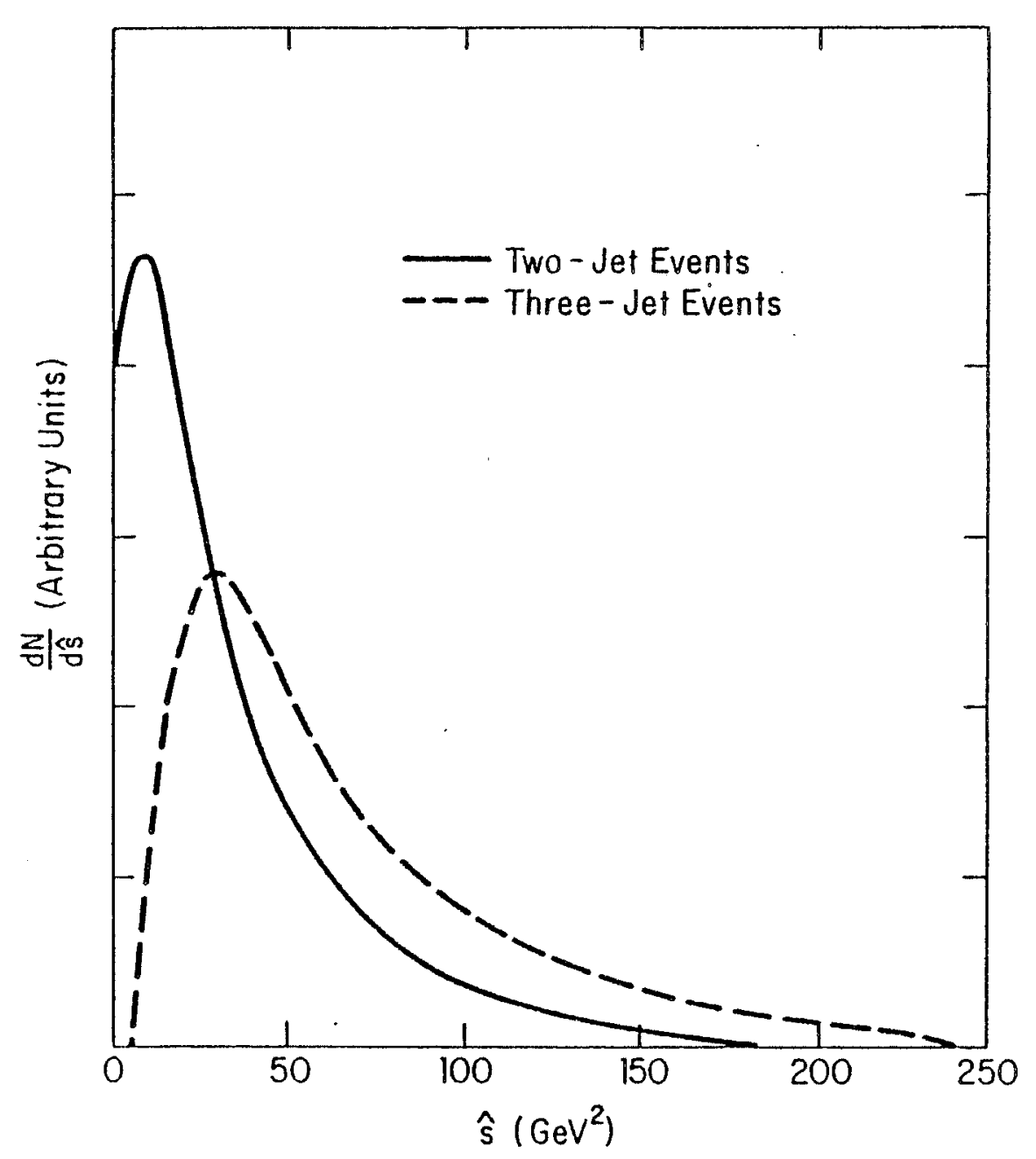

Figure VI-19

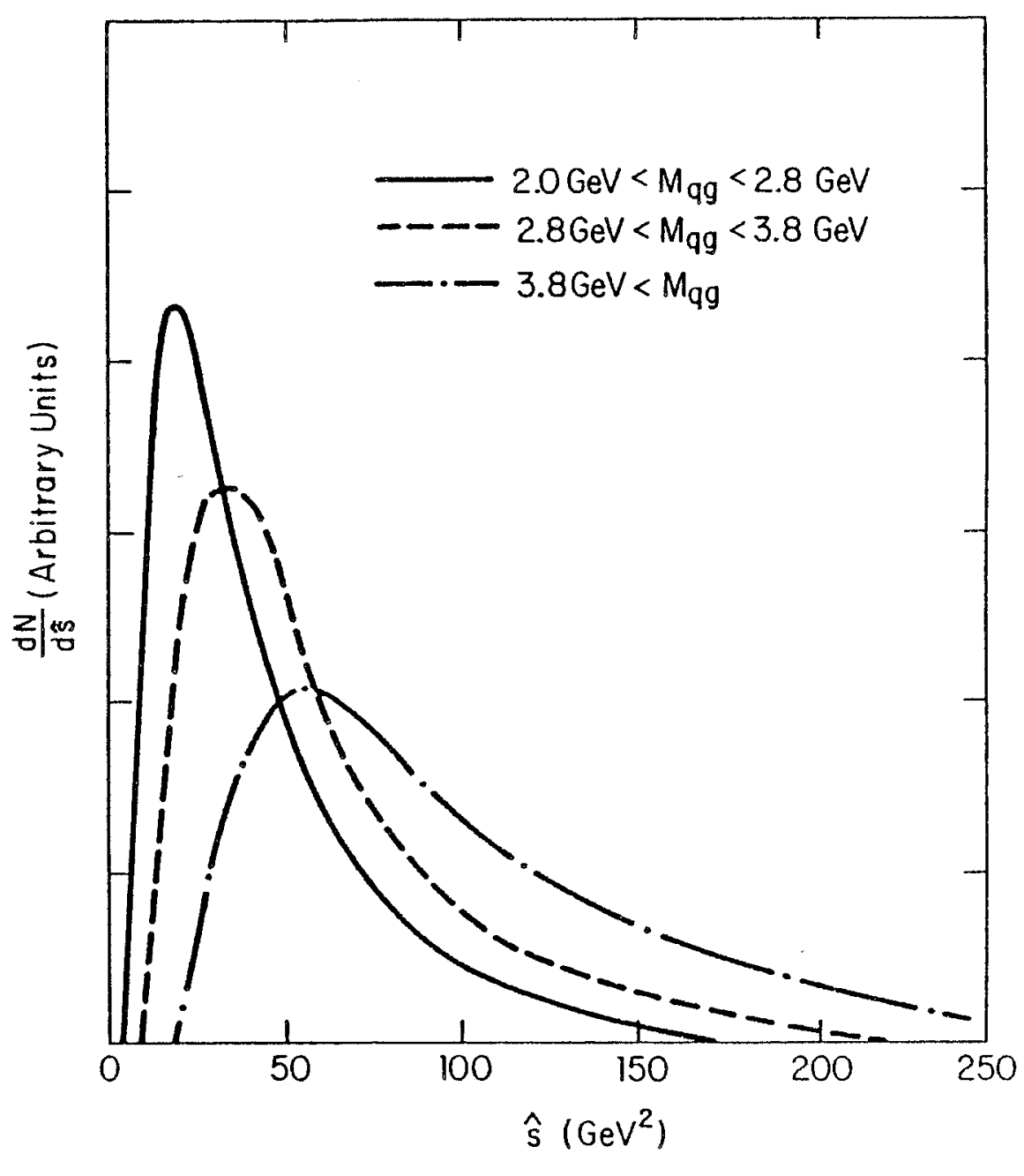

Figure VI-20(a) 


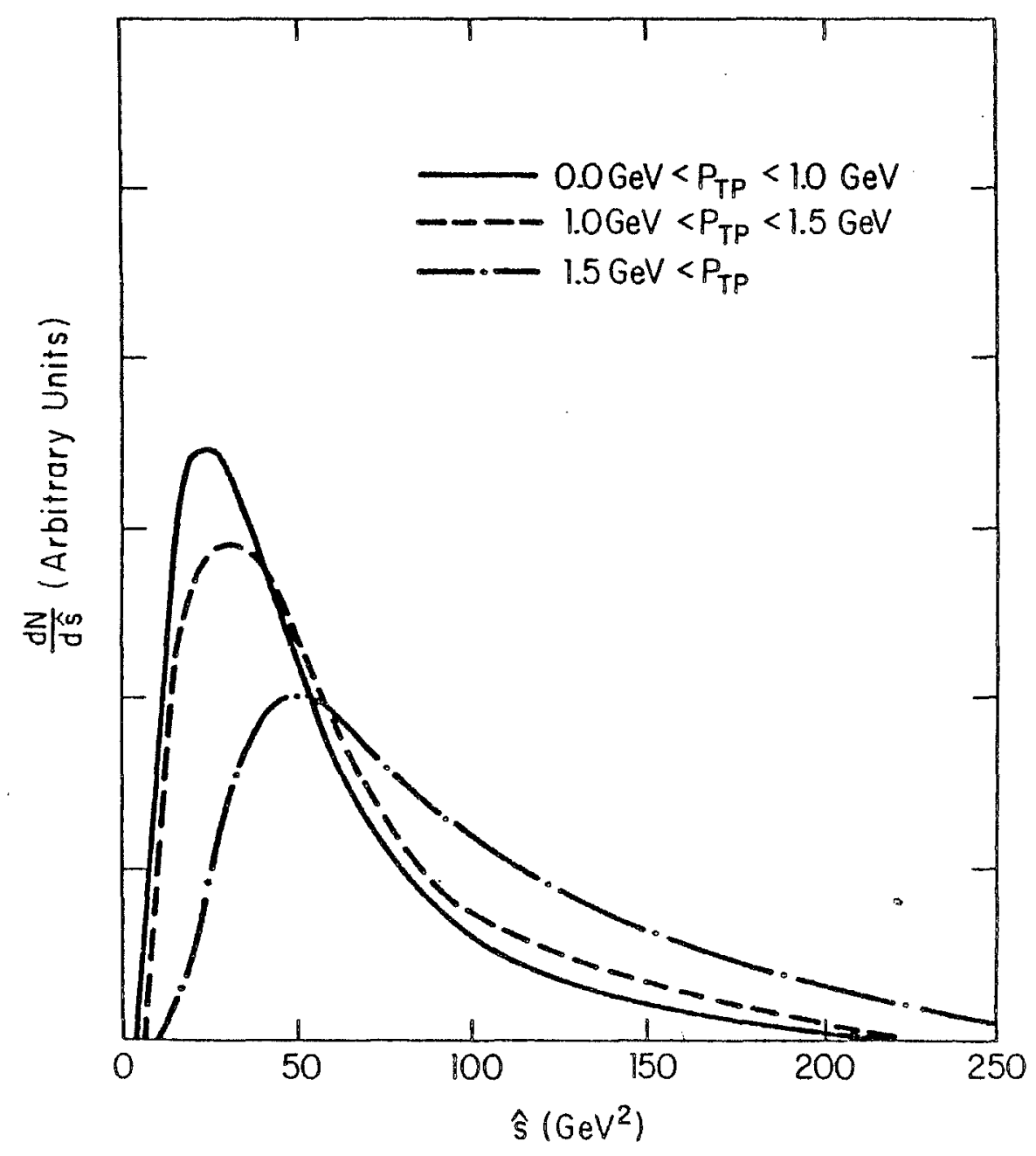

Figure VI-20(b)

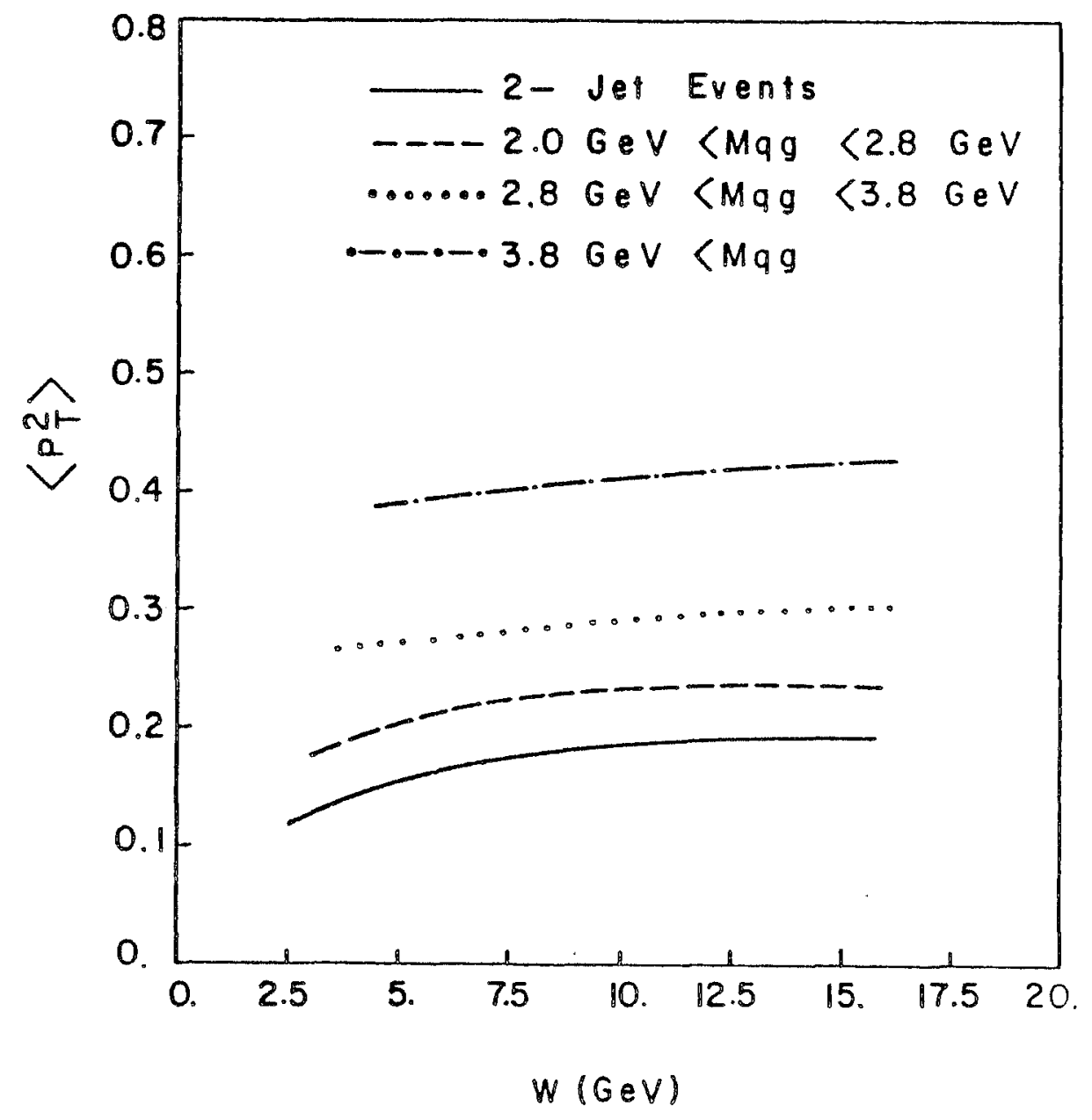

Figure VI-2I 

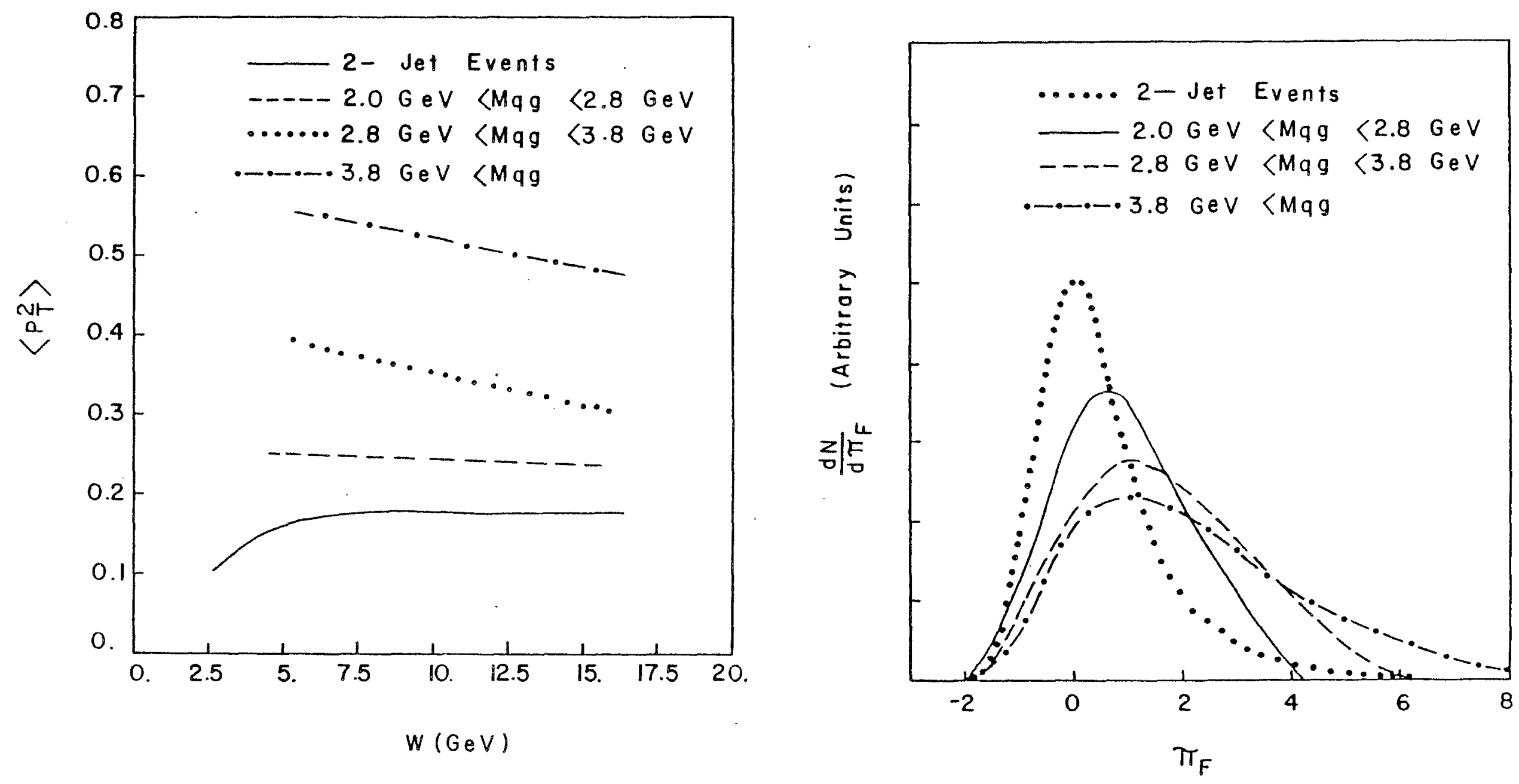

Figure VI-22 


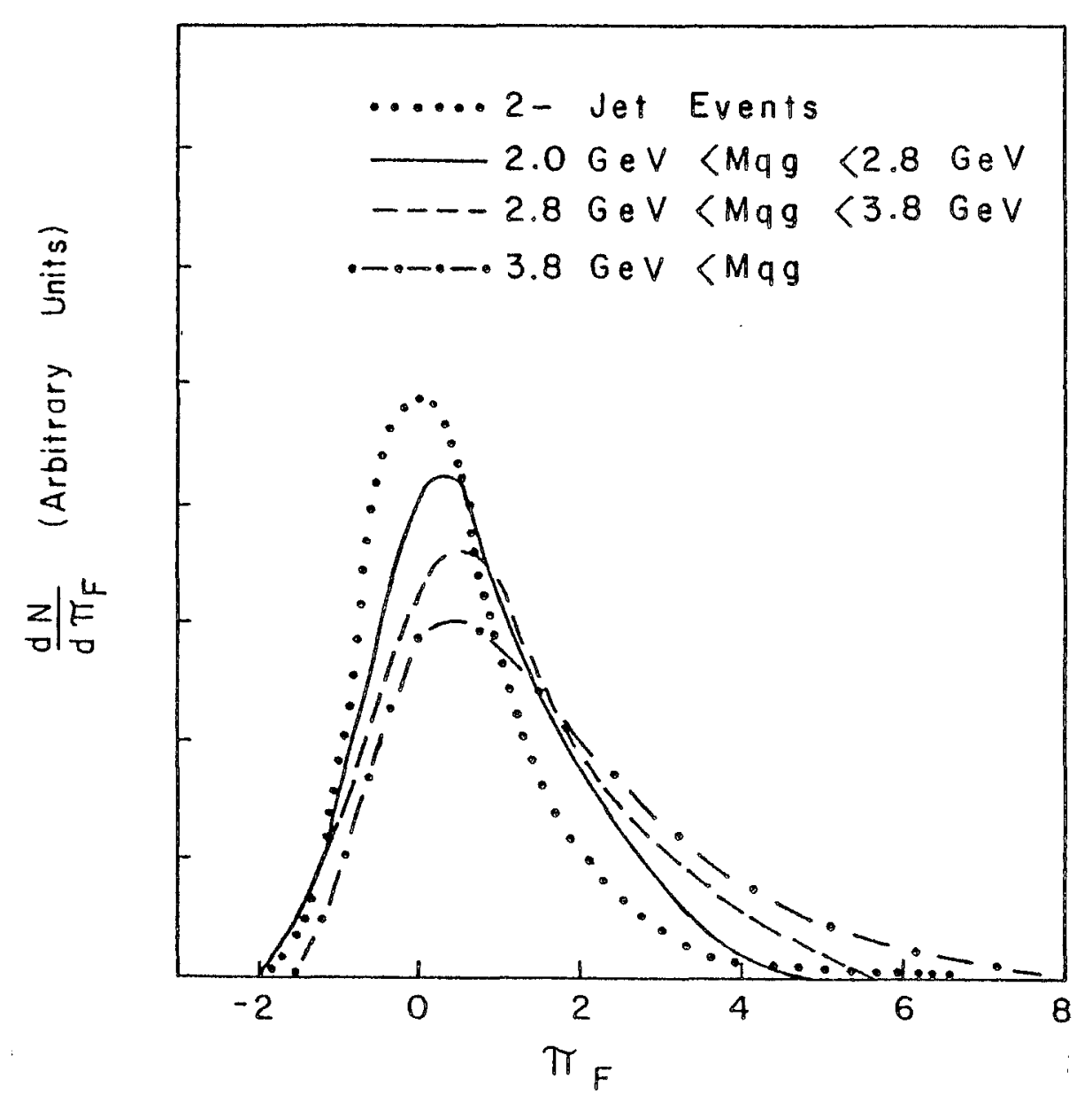

Figure VI-24

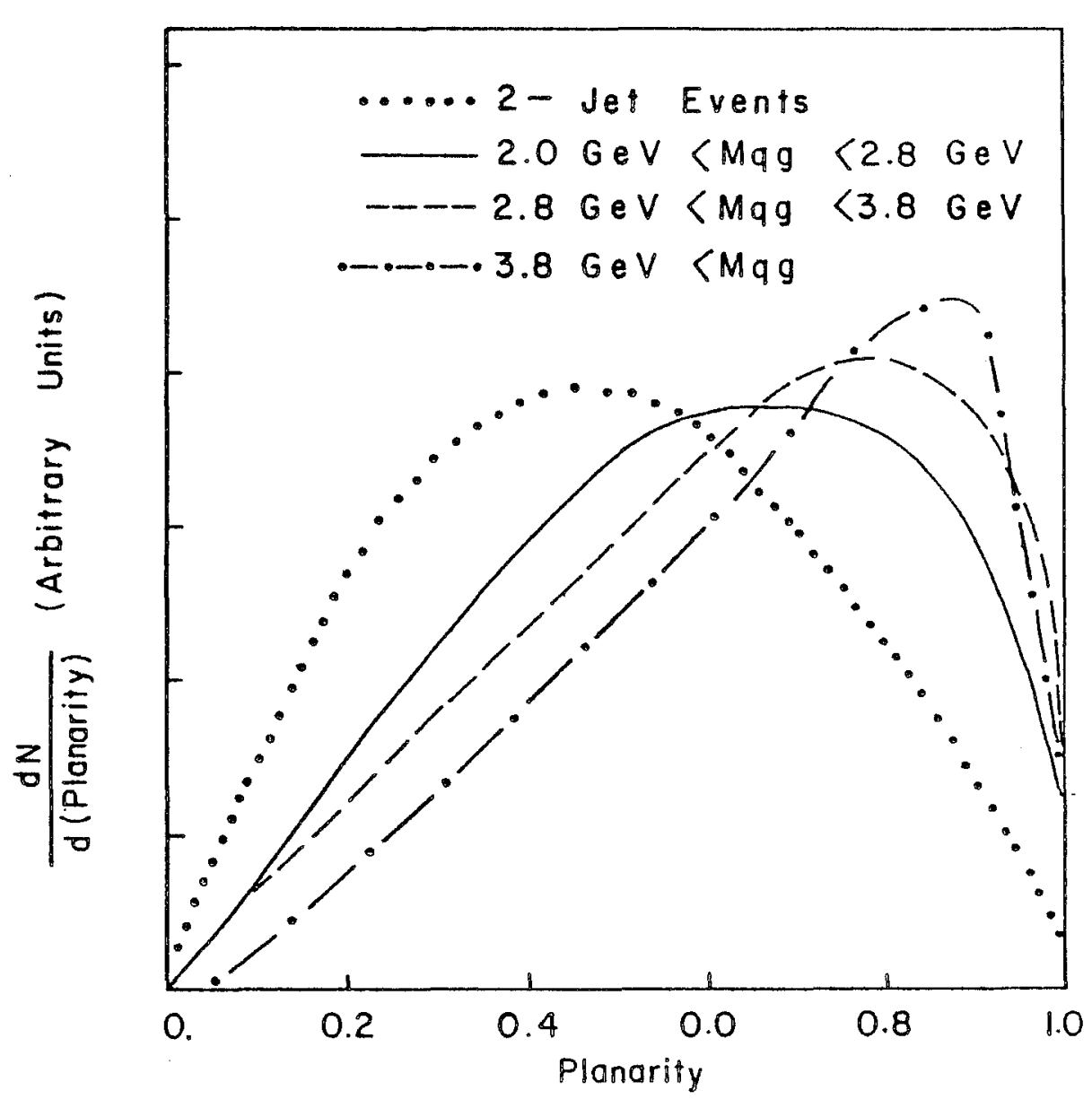

Figure VI-25 

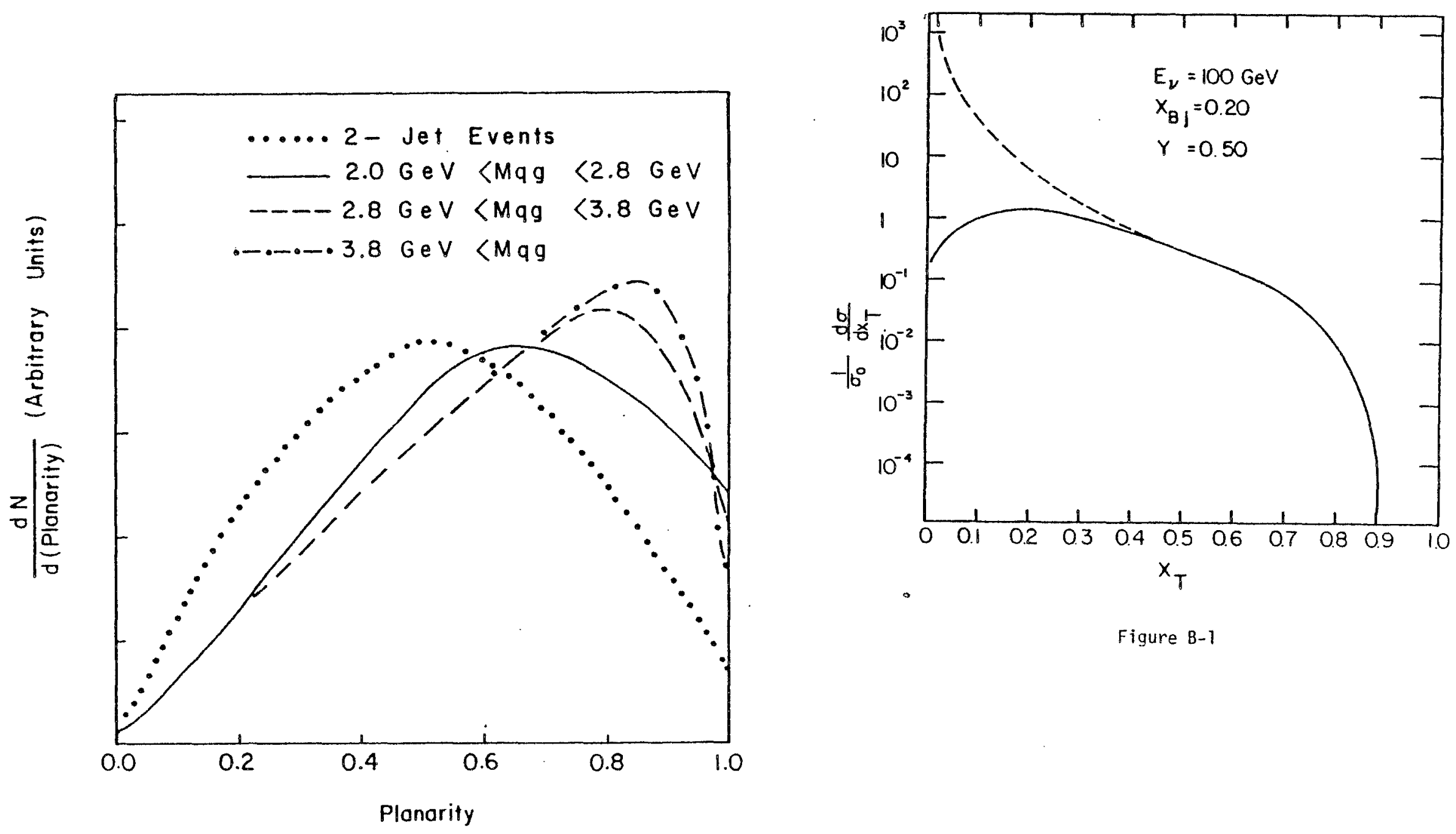

Figure 8-1

Figure VI-26 


\section{Table I-1: The Known Quarks}

$\begin{array}{ccc}\text { Name } & \text { Charge }(e) & \text { Mass }(\mathrm{GeV}) \\ \text { up } & +2 / 3 & <0.02 \\ \text { down } & -1 / 3 & <0.02 \\ \text { strange } & -1 / 3 & \approx 0.3 \\ \text { charm } & +2 / 3 & \approx 1.5 \\ \text { bottom } & -1 / 3 & \approx 5\end{array}$

The five known quarks have electric charges which are integral multiples of $1 / 3$ e (the proton charge). The masses quoted are current masses which describe how quarks behave in hiyh momentum transfer processes. These are to be distinguished from constituent masses which describe how quarks behave in bound states. 


\section{Table III-1: Kinematic Resolutions}

$\begin{array}{ccc}\text { Variable } & \text { Mean Value } & \text { Width } \\ E_{v} & 0.02 & 0.13 \\ E_{H} & 0.03 & 0.20 \\ Q^{2} & 0.02 & 0.16 \\ W & -0.02 & 0.17 \\ \gamma_{H} & 0.07 & 0.19\end{array}$

For each of the listed variables, the mean value and rms width of the resolution function described in Chapter III, section 4 is given. The corresponding resolution functions are plotted in figures III-12 through III-16. 


$$
\text { Table Vol: } \pi \text { By Multiplicity }
$$

$\begin{array}{lcccc}n_{F, B} & \left\langle\pi_{F}\right\rangle & \pi_{F} \text { Width } & \left\langle\pi_{B}\right\rangle & \pi_{B} \text { Width } \\ 1 & 0.38 \pm 0.04 & 1.10 & 0.19 \pm 0.03 & 0.90 \\ 2,3 & 0.24 \pm 0.02 & 0.96 & 0.19 \pm 0.02 & 0.93 \\ 4,5 & 0.01 \pm 0.03 & 0.98 & 0.07 \pm 0.03 & 0.86 \\ 6,7 & 0.00 \pm 0.06 & 0.97 & 0.09 \pm 0.06 & 0.93 \\ 8,9 & -0.49 \pm 0.13 & 0.74 & 0.12 \pm 0.11 & 0.92\end{array}$

For each range of contributing multiplicity, $n_{F, B}$, the average value of the $\pi_{F}$ and $\pi_{B}$ and the rms widths of the $\pi_{F}$ and $\pi_{B}$ distributions are presented. No $\left|x_{F}\right|$ cut was imposed on the tracks and no $W^{2}$ cut was imposed on the events. 
Table V-2: Some High $\pi$ Statistics

(a) Number of Events Observed, $W^{2}>50 \mathrm{GeV}^{2}$

$\begin{array}{lccccc}\pi \text { range: } & 1.6-2.0 & 2.0-2.4 & 2.4-3.0 & 3.0-4.0 & 4.0-10.0 \\ \pi_{F}, P<0.6 & 59 & 39 & 24 & 18 & 7 \\ \pi_{F}, P>0.6 & 40 & 40 & 35 & 34 & 30 \\ \pi_{B}, P<0.6 & 53 & 25 & 16 & 7 & 3 \\ \pi_{B}, P>0.6 & 36 & 36 & 19 & 15 & 6\end{array}$

(b) Symmetric Predictions for $\pi_{F}, W^{2}>50 \mathrm{GeV}^{2}$

$\begin{array}{rrrrrr}P<0.6 & 53.1 & 22.3 & 13.3 & 5.2 & 2.0 \\ P>0.6 & 36.0 & 32.0 & 15.8 & 11.1 & 4.1\end{array}$

(c) Net Number of $\pi_{F}$ Events, $\mathrm{W}^{2}>50 \mathrm{GeV}^{2}$

$$
\begin{array}{rrrrrr}
P<0.6 & 5.9 \pm 10.6 & 16.7 \pm 7.8 & 10.7 \pm 6.1 & 12.8 \pm 4.8 & 5.0 \pm 3.0 \\
P>0.6 & 4.0 \pm 8.7 & 8.0 \pm 8.7 & 19.2 \pm 7.1 & 22.9 \pm 6.7 & 25.9 \pm 5.8 \\
\text { A11 P } & 9.9 \pm 13.7 & 24.7 \pm 11.5 & 29.9 \pm 9.4 & 35.9 \pm 8.2 & 30.9 \pm 6.5
\end{array}
$$


Table V-2, continued

(d) Number of Events Observed, $\mathrm{W}^{2}<50 \mathrm{GeV}^{2}$

$\begin{array}{lccccc}\pi \text { range: } & 1.6-2.0 & 2.0-2.4 & 2.4-3.0 & 3.0-4.0 & 4.0-10.0 \\ \pi_{F}, P<0.6 & 26 & 11 & 2 & 2 & 0 \\ \pi_{F}, P>0.6 & 33 & 17 & 19 & 13 & 4 \\ \pi_{B}, P<0.6 & 22 & 19 & 3 & 1 & 1 \\ \pi_{B}, P>0.6 & 31 & 17 & 16 & 11 & 6\end{array}$

(e) Symmetric Predictions for $\pi_{F}, \mathrm{~W}^{2}<50 \mathrm{GeV}^{2}$

$\begin{array}{rrrrrr}P<0.6 & 19.0 & 16.9 & 2.4 & .0 .7 & 0.7 \\ P>0.6 & 26.8 & 14.4 & 12.8 & 7.9 & 3.9\end{array}$

(f) Net Number of $\pi_{F}$ Events, $W^{2}<50 \mathrm{GeV}^{2}$

$$
\begin{array}{rrrrrr}
P<0.6 & 7.0 \pm 6.7 & -5.1 \pm 5.2 & -0.4 \pm 2.1 & 1.3 \pm 1.6 & -0.7 \pm 0.7 \\
P>0.6 & 6.2 \pm 7.7 & 2.6 \pm 5.6 & 6.2 \pm 5.6 & 5.1 \pm 4.6 & 0.1 \pm 2.8 \\
\text { A11 P } & 13.2 \pm 10.2 & -2.5 \pm 7.6 & 5.8 \pm 6.0 & 6.4 \pm 4.9 & -0.6 \pm 2.9
\end{array}
$$


Table $V-3$ : Minimum $\left\langle\left|P_{T}\right|>\right.$ for $\pi_{F(B)} \geqslant 3.0$

$\begin{array}{cc}n_{F(B)} & \left\langle\left|P_{T}\right|\right\rangle_{\min }(\mathrm{GeV}) \\ 1 & 1.070 \\ 2 & 0.850 \\ 3 & 0.753 \\ 4 & 0.695 \\ 5 & 0.655 \\ 6 & 0.626 \\ 7 & 0.603 \\ 8 & 0.585\end{array}$

Here we have calculated the minimum average $\left|P_{T}\right|$. of contributing tracks, $\left\langle\left|P_{T}\right|\right\rangle_{\min }$, required to give $\pi_{F(B)} \geqslant 3.0$ for contributing multiplicities, $n_{F(B)}$, between 1 and 8 . In the definition of $\pi_{F(B)}$, equation $V_{0} 3$, we have taken $P_{T O}=0.320 \mathrm{GeV}$ and $A=4 \mathrm{GeV}^{-1}$, as is used throughout our analysis. 
Table VI-1: Event Characteristics

\begin{tabular}{|c|c|c|c|c|c|c|c|c|}
\hline & Two-jet & Three-jet & Low Mqg & Mid Mqg & High Mqg & Low $\mathrm{P}_{\mathrm{TP}}$ & Mid $P_{T P}$ & High $\mathrm{P}_{\mathrm{TP}}$ \\
\hline$\left\langle W^{2}\right\rangle\left(\mathrm{GeV}^{2}\right)$ & 100 & 113 & 101 & 110 & 135 & 106 & 110 & 132 \\
\hline$\left\langle E_{v}\right\rangle(\mathrm{GeV})$ & 112 & 127 & 112 & 124 & 150 & 117 & 124 & 149 \\
\hline$\langle u\rangle \quad(\mathrm{GeV})$ & 70 & 84 & 71 & 81 & 105 & 75 & 81 & 103 \\
\hline$\left\langle Q^{2}\right\rangle\left(\mathrm{GeV}^{2}\right)$ & 25 & 31 & 25 & 31 & 41 & 27 & 31 & 40 \\
\hline$\langle y\rangle$ & 0.68 & 0.70 & 0.68 & 0.69 & 0.73 & 0.69 & 0.69 & 0.72 \\
\hline$\left\langle x_{B j}\right\rangle$ & 0.19 & 0.20 & 0.19 & 0.21 & 0.22 & 0.19 & 0.21 & 0.22 \\
\hline$\left\langle E_{0}\right\rangle$ & 0.19 & 0.30 & 0.24 & 0.30 & 0.39 & 0.27 & 0.29 & 0.38 \\
\hline$\langle\hat{\mathrm{S}}\rangle \quad\left(\mathrm{GeV}^{2}\right)$ & 39 & 68 & 49 & 66 & 99 & 57 & 69 & 98 \\
\hline
\end{tabular}

In the QCD Monte Carlo, the $\mathrm{W}^{2}>50 \mathrm{GeV}^{2}$ events have average values of kinematic variables as given. The events have been split into two- and three-jet samples, and the three-jet sample further split according to $\mathrm{Mqg}$ and $\mathrm{P}_{\mathrm{TP}}$. The three ranges of $\mathrm{Mqg}$ are $2 \mathrm{GeV}-2.8 \mathrm{GeV}, 2.8 \mathrm{GeV}-3.8 \mathrm{GeV}$, and greater than $3.8 \mathrm{GeV}$. The three ranges of $\mathrm{P}_{\mathrm{TP}}$ are less than $1 \mathrm{GeV}, 1 \mathrm{GeV}-1.5 \mathrm{GeV}$ and greater than $1.5 \mathrm{GeV}$. Each of these subsamples contains about one third of the three-jet Monte Carlo events. 